\title{
Role of PIKfyve in macrophage and neutrophil immune response
}

\author{
By \\ Roya Monica Dayam \\ A dissertation \\ submitted to Ryerson University \\ in partial fulfillment of the requirements for the degree \\ of \\ Doctor of Philosophy \\ in \\ the program of Molecular Science \\ RYERSON UNIVERSITY \\ Toronto, Ontario, 2017 \\ (C) Roya Monica Dayam,2017
}




\section{Author's declaration}

I hereby declare that I am the sole author of this dissertation. This is a true copy of the dissertation, including any required final revisions, as accepted by my examiners.

I authorize Ryerson University to lend this dissertation to other institutions or individuals for the purpose of scholarly research.

I further authorize Ryerson University to reproduce this dissertation by photocopying or by other means, in total or in part, at the request of other institutions or individuals for the purpose of scholarly research.

I understand that my dissertation may be made digitally available to the public. 


\section{Acknowledgements}

I would like to convey my heartfelt gratitude and sincere appreciation to my supervisor Dr. Roberto Botelho, who has constantly inspired, encouraged, guided and supported me throughout my undergraduate and graduate studies.

My sincere thanks go to present and past members of The Botelho lab, especially Dr. Mathew Gray, Dr. Amra Saric, Victoria Hipolito, and Zechariah Martin. I am very grateful for your help, guidance, moral support and encouragement.

I would like to extend my sincerest thanks and gratitude to my thesis committee members, Dr. Costin Antonescu and Dr. Jeffery Fillingham for their guidance and support and providing interesting and valuable ideas and feedback.

I would like to additionally thank our collaborators Dr. Michael Glogauer, and Chun X. Sun from Faculty of Dentistry, University of Toronto; and Dr. Mauricio Terebiznik and Akriti Prashar from the Department of Cell and Systems Biology, University of Toronto. I also would like to express my very profound gratitude to my husband Alex Kuresh, for his unconditional love, support and encouragement through all these years of my graduate studies. I wouldn't have been able to complete my dissertation without you by my side. Also thanks to my precious and beautiful daughters, my biggest blessing, Kiana Kuresh and Kaitlin Kuresh, for their love, understanding, patience and support while I finished my degree. Thanks for being my inspiration and motivation in the past few years, spending hours in the office while mommy had to work in the lab. Your hugs and kisses were the solution to all my problems throughout these years. I love you both. 
I am extremely thankful to my family, my mom, dad, and brothers who have always encouraged me to push through the difficult times; your persistent belief in me helped me to believe in myself. Thanks for always being there when I need you and helping me achieve my goals. 


\title{
Role of PIKfyve in macrophage and neutrophil immune response
}

\author{
Roya Monica Dayam, 2017 \\ $\mathrm{PhD}$, Molecular Science, Ryerson University
}

\begin{abstract}
Solid particles such as pathogens, dying cells, and debris are engulfed by macrophages and neutrophils and sequestered into a phagosome. Phagosomes fuse with early and late endosomes and ultimately with lysosomes to mature into phagolysosomes, a process known as phagosome maturation. The formation of highly acidic and degradative phagolysosomes plays an important role in degradation of the internalized particle. We employed siRNA and pharmacological tools to demonstrate that phosphatidylinositol-3,5-bisphosphate $\left[\mathrm{PI}(3,5) \mathrm{P}_{2}\right]$, synthesized by the PIKfyve lipid kinase, is required for phagosome maturation. However, the mechanism by which $\mathrm{PI}(3,5) \mathrm{P}_{2}$ controls phagosome maturation remained uncharacterized. We hypothesized that $\mathrm{PI}(3,5) \mathrm{P}_{2}$ may control phagosome-lysosome fusion partly by stimulating TRPML1, a Iysosomal $\mathrm{Ca}^{2+}$ channel gated by $\mathrm{PI}(3,5) \mathrm{P}_{2}$. Upon opening of the channel, lysosomal $\mathrm{Ca}^{2+}$ would diffuse and trigger phagosome-lysosome fusion since $\mathrm{Ca}^{2+}$ is known to induce membrane fusion post-docking of SNARE proteins. In addition, we also demonstrated that the lipid kinase PIKfyve coordinates the neutrophils immune response by controlling phagosome maturation and regulating Rac GTPase activity. PIKfyve produces both $\mathrm{PI}(3,5) \mathrm{P}_{2}$ and phosphatidylinositol-5-phosphate (PI5P); therefore, it might control phagosome maturation through production of $\mathrm{PI}(3,5) \mathrm{P}_{2}$ and activation of TRPML1 as
\end{abstract}


well as regulates ROS production and chemotaxis through synthesis of PI5P, which leads to the activation of Tiam1, and Rac GTPase. 


\section{Table of contents}

Acknowledgements ................................................................................. iii

Abstract ................................................................................................. v

Table of contents ....................................................................................... vii

List of figures ................................................................................................

List of abbreviations ................................................................................... xiii

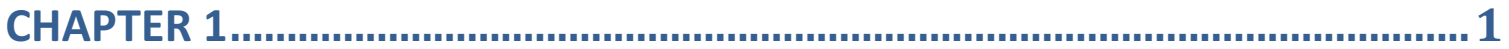

Introduction .............................................................................. 1

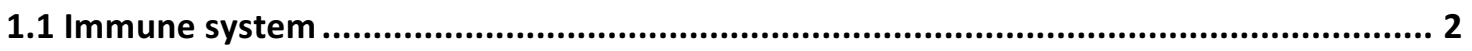

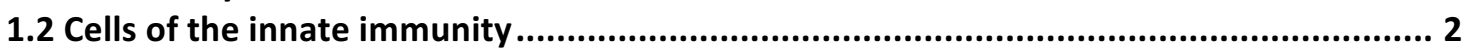

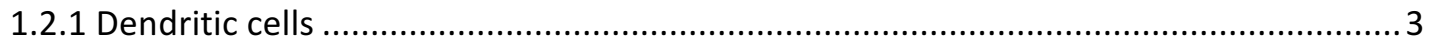

1.2.2 Neutrophils

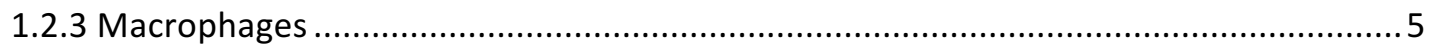

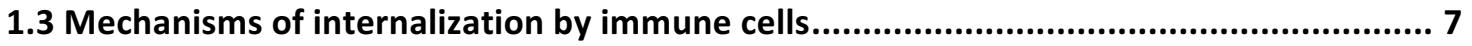

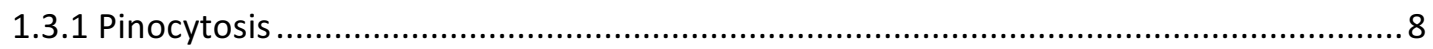

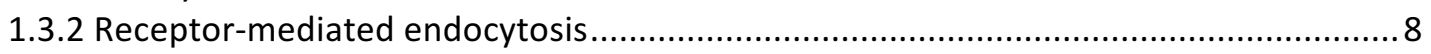

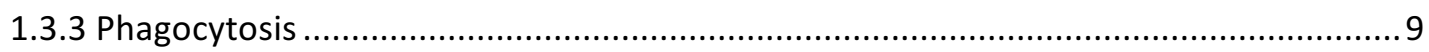

1.4 Lysosomes: small organelles with big responsibilities ................................................10

1.5 Phagocytosis and phagosome maturation in macrophages ..........................................12

1.6 Phosphoinositides: Important spatio-temporal regulators of cell signaling \& intracellular

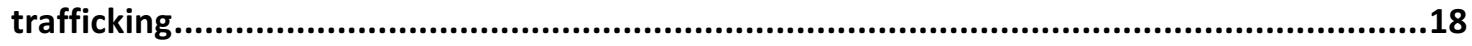

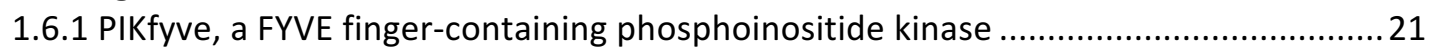

1.7 The lysosomal calcium channel, TRPML1 ...............................................................23

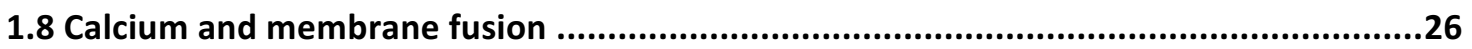

1.9 Phagocytosis and phagosome maturation in neutrophils .........................................27

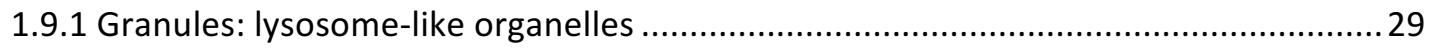

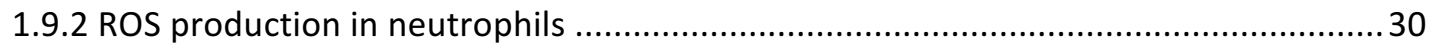

1.9.3 RAC1 and RAC2 small GTPase during neutrophils immune responses ........................ 33

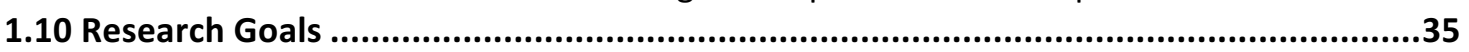

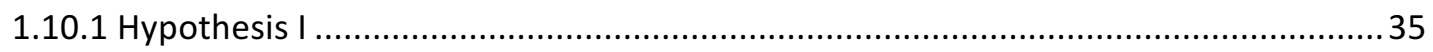

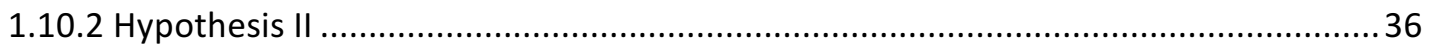

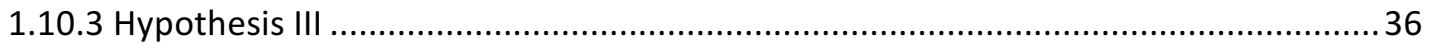

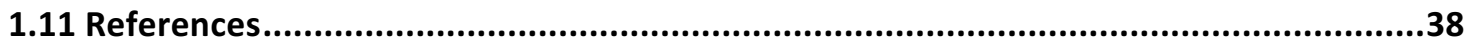

Chapter 2 ............................................................................... 67

2.1 PIKfyve inhibition interferes with phagosome and endosome maturation in macrophages .................................................................. 67

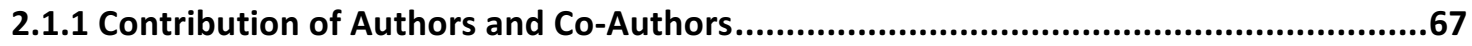

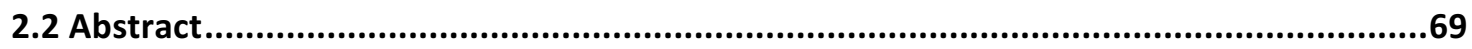




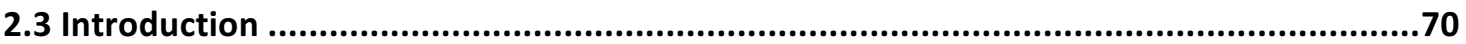

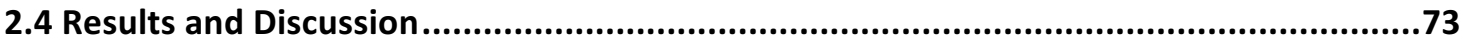

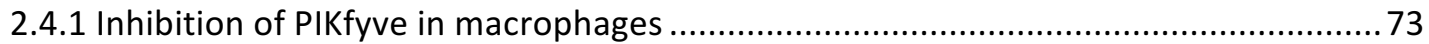

2.4.2 Differential effects on early and late endocytic organelles in PIKfyve inhibited

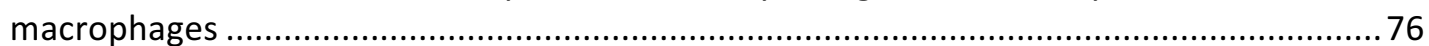

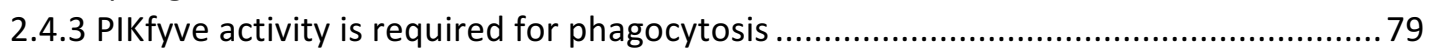

2.4.4 Early phagosome maturation is altered in PIKfyve-blocked macrophages .................. 82

2.4.5 PIKfyve activity is required for phagosomes to acquire lysosomal markers................ 84

2.4.6 PIKfyve is necessary for efficient trafficking of immune complexes to lysosomes......89

2.4.7 Phagosomal and lysosomal acidification does not require PIKfyve.............................91

2.4.8 PIKfyve is important for efficient phagosomal and lysosomal proteolytic activity......93

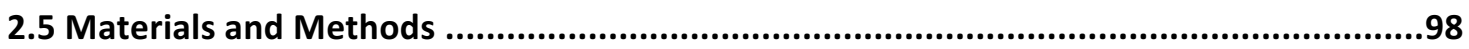

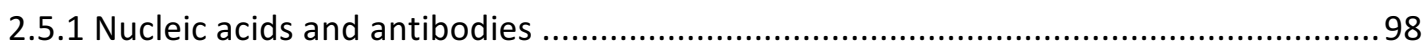

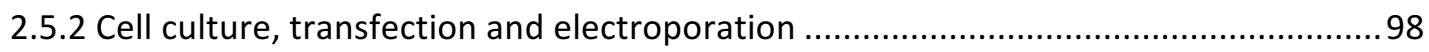

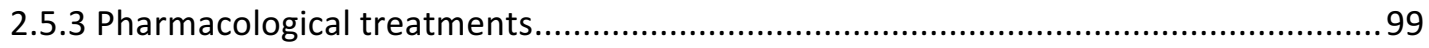

2.5.4 Phagocytosis and phagosome maturation assays .......................................................99

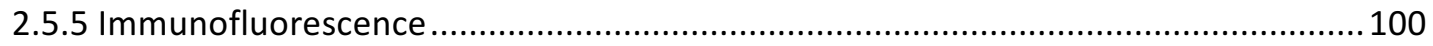

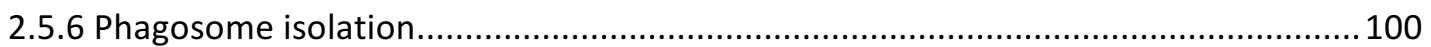

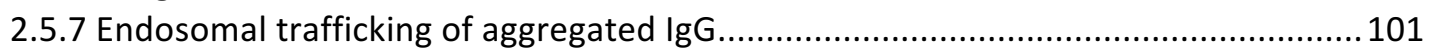

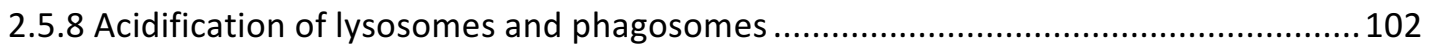

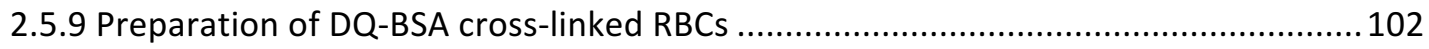

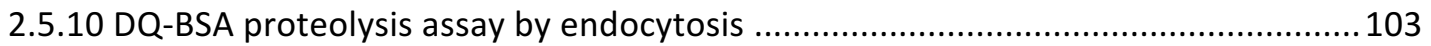

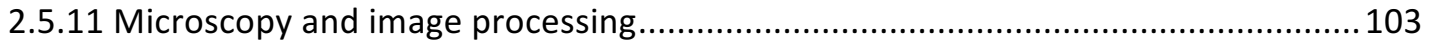

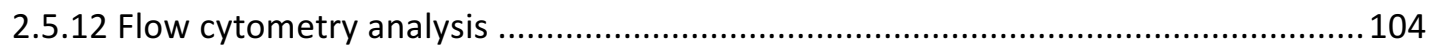

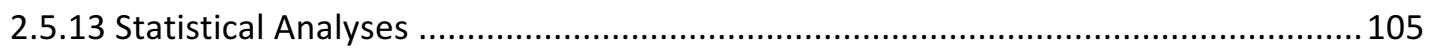

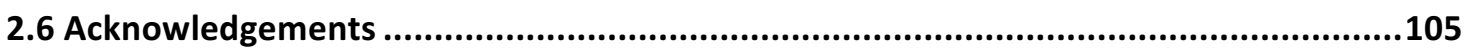

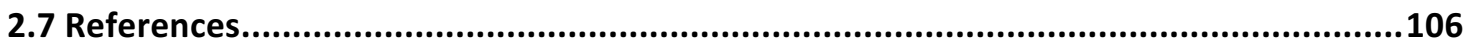

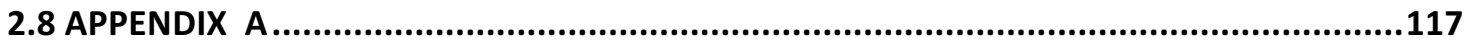

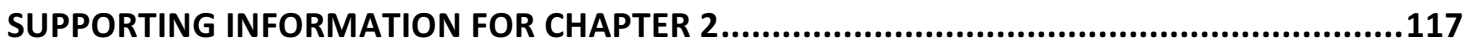

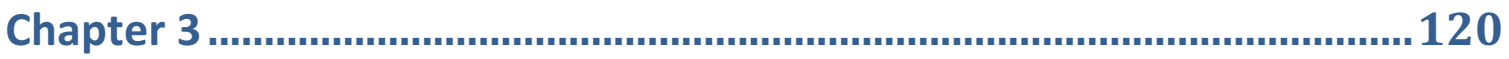

3.1 The phosphoinositide-gated lysosomal $\mathrm{Ca}^{2+}$ channel, TRPML1, is

required for phagosome maturation ................................................... 120

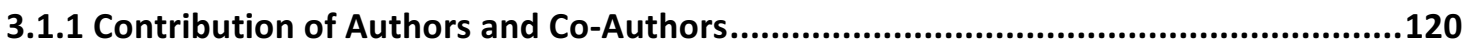

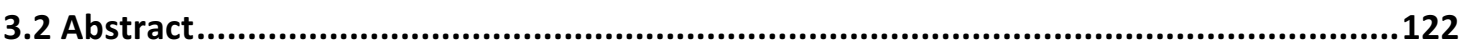

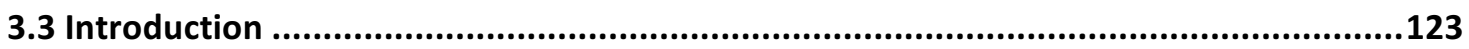

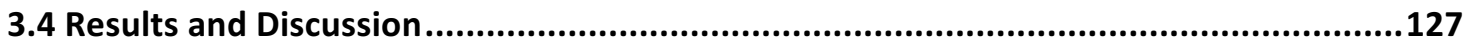

3.4.1 TRPML1 silencing in macrophages impacts lysosome morphology..........................127

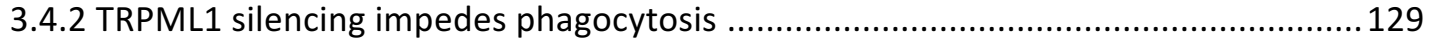

3.4.3 TRPML1 activity does not affect phosphoinositide dynamics on phagosomes ..........132

3.4.4 TRPML1 silencing impairs phagosome maturation and bactericidal activity .............135

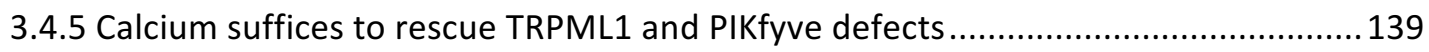

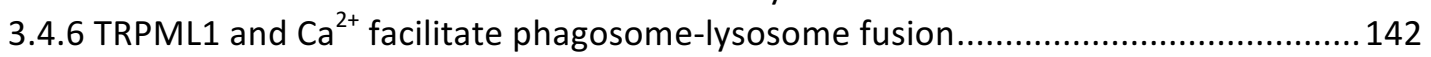

3.4.7 Calcium dynamics during phagosome maturation ..................................................146

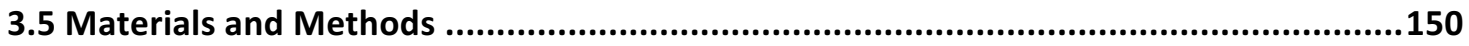

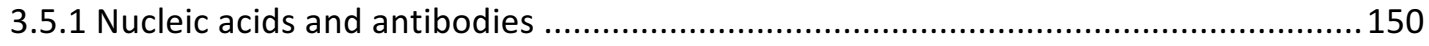

3.5.2 Cell culture, transfection and gene silencing ...................................................... 150 


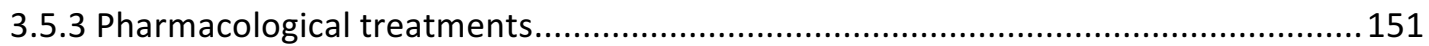

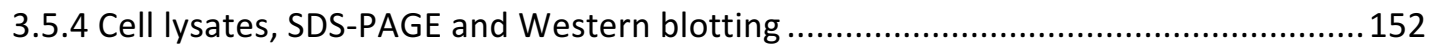

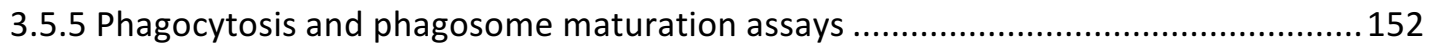

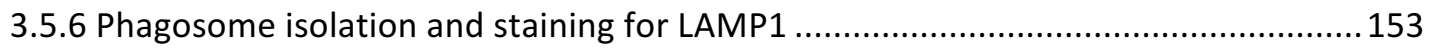

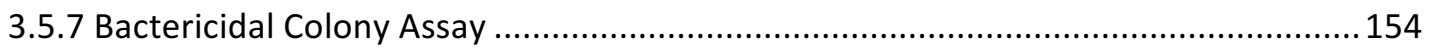

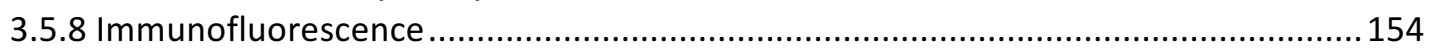

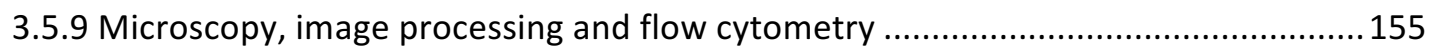

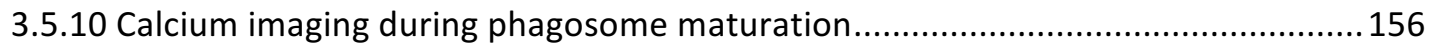

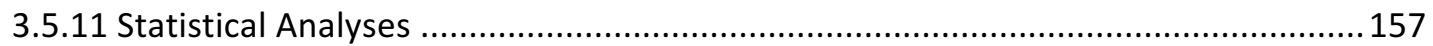

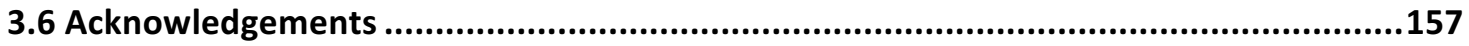

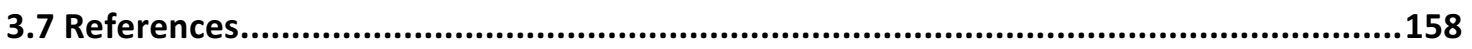

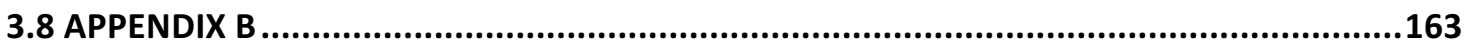

SUPPORTING INFORMATION FOR CHAPTER 3

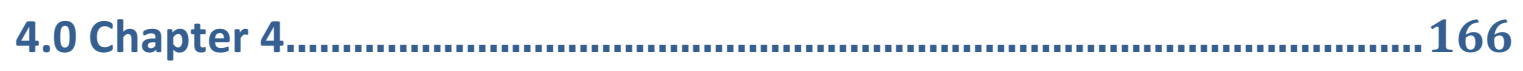

The lipid kinase PIKfyve coordinates the neutrophil immune response through the activation of the Rac GTPase ..........................................166

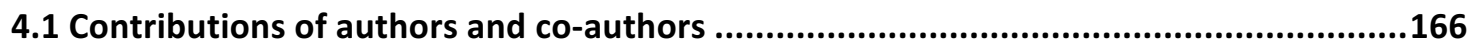

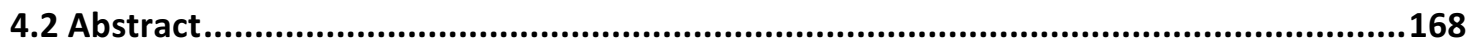

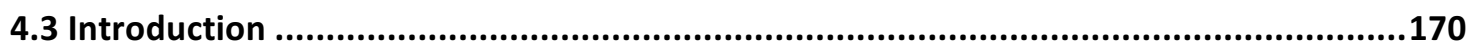

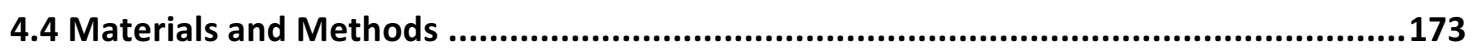

4.4.1 Bone marrow-derived neutrophil isolation and stimulation ..................................173

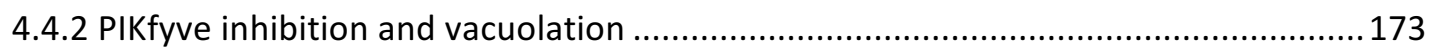

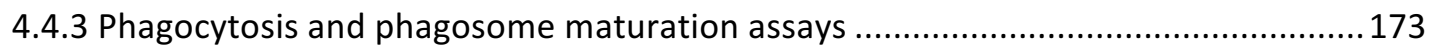

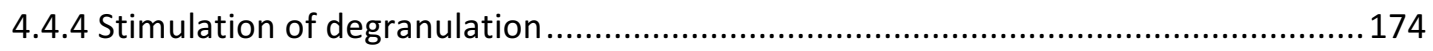

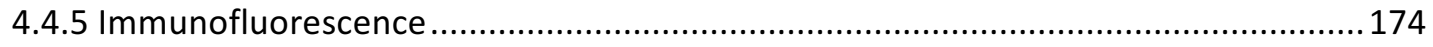

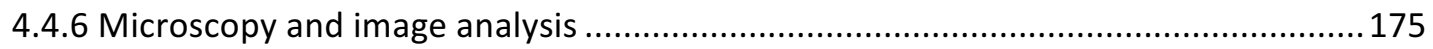

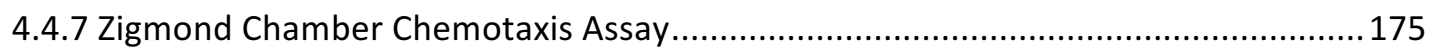

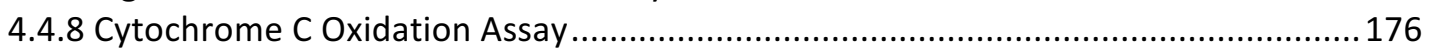

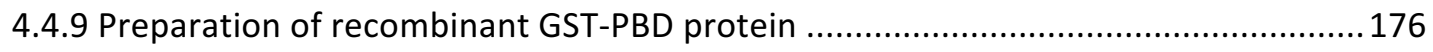

4.4.10 Affinity precipitation of GTP-bound Rac GTPase and Western blotting ..................177

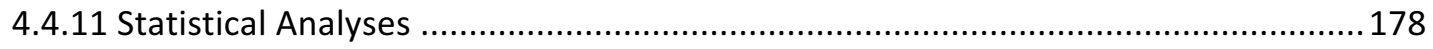

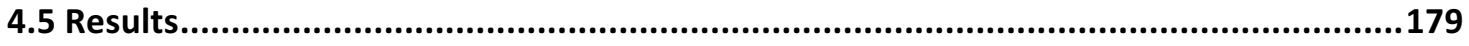

4.5.1 Lysosomes but not granules vacuolate in PIKfyve-inhibited neutrophils ...................179

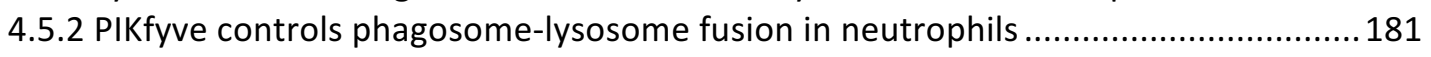

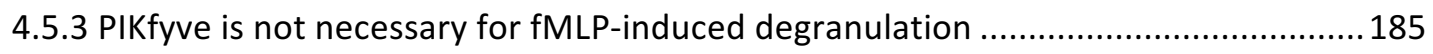

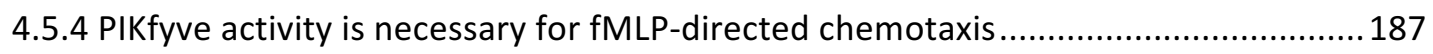

4.5.5 PIKfyve activity is necessary for fMLP-induced ROS production ...............................189

4.5.6 PIKfyve activity is necessary for fMLP-induced Rac activation ................................ 190

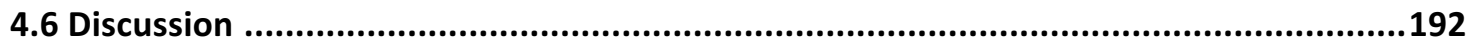

4.6.1 Impact of PIKfyve on neutrophil lysosomes and granules .......................................192

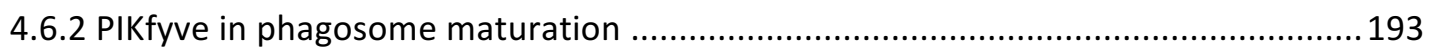

4.6.3 PIKfyve activity is necessary for Rac activation in neutrophils ............................... 194

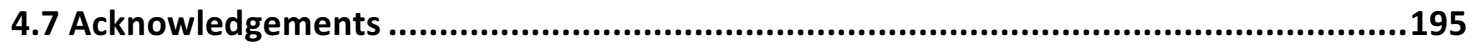

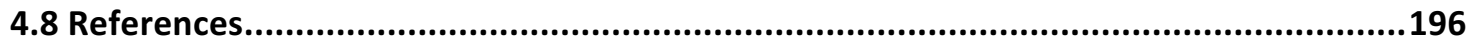

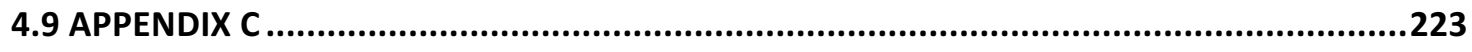

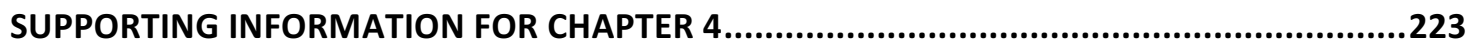




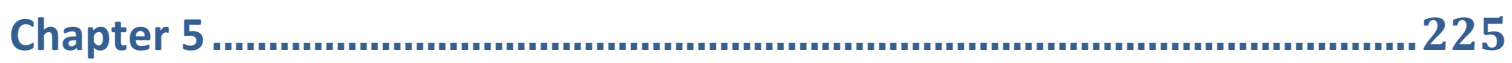

Concluding remarks and future directions .................................... 225

5.1 PIKfyve inhibition interferes with phagosome and endosome maturation in macrophages.

5.2 The phosphoinositide-gated lysosomal $\mathrm{Ca}^{2+}$ channel, TRPML1, is required for phagosome maturation .....................................................................................................227

5.3 The lipid kinase PIKfyve coordinates the neutrophil immune response through the

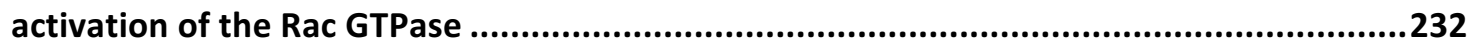

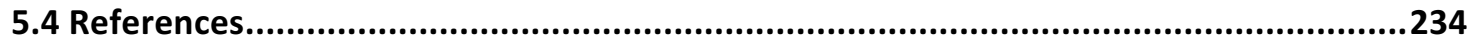




\section{List of figures}

FigURE 1.1: FC $\gamma$ RECEPTOR MEDIATED PHAGOCYTOSIS IN MACROPHAGES. 14

FIGURE 1.2: THE PROCESS OF PHAGOSOME MATURATION IN MACROPHAGES 17

Figure 1.3 PhosphatidylinOSITOL AND ITS SEVEN PHOSPHORYLATED SPECIES.

FIGURE 1.4: THE PAS COMPLEX: 22

Figure 1.5: Transient Receptor Potential Mucolipin-1 (TRPML-1) Structure .25

FIGURE 1.6: THE ROLE OF CALCIUM IN MEMBRANE FUSION 27

FigURE 1.7: FUSION OF GRANULES WITH PHAGOSOME AND PRODUCTION OF REACTIVE OXYGEN SPECIE 32

FIGURE 1.8: RAC-GTPASES BEHAVE LIKE MOLECULAR SWITCHES: 34

FigURE 2.1: INHIBITION OF PIKFYVE AND VACUOLATION IN MACROPHAGES 75

FiguRE 2.2: THE IDENTITY OF VACUOLES IN PIKFYVE-INHIBITED MACROPHAgES. 79

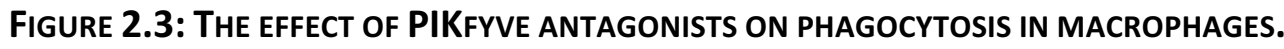
81

Figure 2.4: PIKfyve inhibition affects the kinetics of Ptdins(3)P on Phagosomes. .83

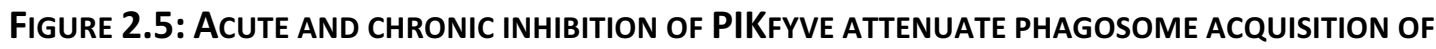

LAMP1. 88

FIGURE 2.6: PIKFYVE IS REQUIRED FOR EFFICIENT TRAFFICKING OF IMMUNE COMPLEXES TO LYSOSOMES.

Figure 2.7: Phagosomes AND lysosomes ACIDIFY IndePENDENTLY of PIKfyVE. 92

FIGURE 2.8: PIKFYVE INHIBITION IMPAIRS PROTEOLYTIC ACTIVITY OF PHAGOSOMES AND LYSOSOMES. . 97

FIGURE S 2.1: YM201636 AND APILIMOD TREATMENTS HINDER PHAGOSOME MATURATION 118

FIGURE S2.2: PIKFYVE INHIBITION IMPAIRS ACCUMULATION OF FLUID-PHASE PINOCYTOSIS. 119

FIGURE 3.1: TRPML1 SILENCING AND ITS EFFECT ON LYSOSOMES. 128 
FIGURE 3.2: THE EFFECT OF TRPML1 SILENCING ON PHAGOCYTOSIS IN MACROPHAGES.

FIGURE 3.3: FPHOSPHATIDYLINOSITOL-3-PHOSPHATE DYNAMICS ON PHAGOSOMES IN TRPML1SILENCED MACROPHAGES.

FigURE 3.4: THE EFFECT OF TRPML1 AND PIKFYVE LOSS OF FUNCTION ON PHAGOSOME MATURATION AND BACTERIAL KILLING.

FIGURE 3.5: THE EFFECTS OF IONOMYCIN AND BAPTA-AM ON PHAGOSOME ACQUISITION OF LAMP1 IN PIKfyVe AND TRPML1-DEFICIENT CELLS. 141

FIGURE 3.6: FLOW CYTOMETRY AND MICROSCOPY OF ISOLATED PHAGOSOMES STAINED FOR LAMP1.146

FigURE 3.7: The EFFECT OF PHAgOCYTOSIS ON FLUO4-AM FLUORESCENCE IN RAW MACROPHAGES.149

Figure S 3.1: THE EFFECT OF PIKfYVE AND TRPML1 on BACTERIAL UPTAKE AND SURVIVAL. 164

Figure S 3.2: FLUO4-AM FLUORESCENCE CHANGES IN LYSOSOMES AND CYTOSOL OF RAW MACROPHAGES. 165

FiguRE 4.1: POTENCY AND KINETICS OF APILIMOD-INDUCED VACUOLATION IN NEUTROPHILS. 180

FigURE 4.2: PIKFYVE INHIBITION ENGORGES LYSOSOMES BUT NOT GRANULES. 181

FigURE 4.3: PIKFYVE INHIBITION BLOCKS PHAGOSOME-LYSOSOME FUSION. .185

Figure 4.4: PIKfyVE ACTIVITY DOES NOT AFFECT DEGRANULATION. 186

FIGURE 4.5: PIKFYVE ACTIVITY IS IMPORTANT FOR NEUTROPHIL CHEMOTAXIS. 189

FigURE 4.6: PIKfYVE ACTIVITY IS ESSENTIAL FOR REACTIVE OXYGEN SPECIES GENERATION IN NEUTROPHILS. 190

Figure 4.7: PIKfyVe is ESSENTIAL to RAC GTPASE StimULATION IN NEUTROPHILS. 191

GRANULE MARKER. .224

Figure 5.1: Lysosomes taRgeted genetically encoded CA $^{2+}$ SENSORS, GCAMP3-ML1..... 229 


\section{List of abbreviations}

APCs- antigen presenting cells

ARP2/3- actin related protein2 and 3

DAG- diacylglycerol

DAMPs- damage associated molecular patterns

DCs- dendritic cells

EEA1- early endosome autoantigen

ENTH- epsin N-terminal homology

ER- endoplasmic reticulum

ERK- extracellular signal-regulated kinase

ESCRT- endosomal sorting complexes required for transport

FACS- Fluorescence-activated cell sorting

fMLP- N-Formylmethionyl-leucyl-phenylalanine

FYVE domain- Fab 1 (yeast orthologue of PIKfyve), YOTB, Vac 1 \& EEA1

GAP- GTPase Activating Protein

GCaMP-green fluorescent protein (GFP), calmodulin, M13 peptide

GEF- Guanine exchange factor

GSTs- Glutathione S-transferases

HOPS- homotypic fusion and protein sorting

HOCl- hypochlorous acid 
IgG- Immunoglobulins g

IL- Interleukin

INF- interferon

ITAM- immunoreceptor tyrosine based activation motif

LAM- lipoarabinomannan

LAMP- Iysosomal associated membrane protein

LIMPs- lysosomal membrane proteins

MHC- major histocompatibility complex

MLIV- type 4 mucolipidosis

MLCK- myosin light chain kinase

ML-SA1- mucolipin synthetic agonist 1

MMP9- Matrix metallopeptidase 9

Mtb- Mycobacterium tuberculosis

M6PR- mannoses 6 phosphate receptor

MPO- myeloperoxidase

mTORC- mechanistic target of rapamycin complex

NADPH- nicotinamide adenine dinucleotide phosphate

NET- neutrophils extracellular trap

NOS- nitric oxide synthase

PAMPs- pathogen associated molecular patterns

PAS- PIKfyve-ArPIKfyve-Sac3

PBD- protein binding domain 
PH domain - Pleckstrin homology domain

PIS- phosphatidyl inositol synthase

PITPs- phosphatidyl inositol transfer proteins

PIM - phosphatidylinositol mannoside

PIKfyve- FYVE finger-containing phosphoinositide kinase

PI3P- phosphatidylinositol 3-phsophate

PI4P- phosphatidylinositol 4-phosphate

PI5P-phosphatidylinositol 5-phosphate

PI3,5P2- phosphatidylinositol 3,5-bisphosphate

PI4,5P2- phosphatidylinositol 4,5-bisphosphate

PI3,4P2- phosphatidylinositol 3,4-bisphosphate

PI3,4,5P2- phosphatidylinositol (3,4,5)-triphosphate

PI3K- phosphatidylinositol 3-Kinase

PIP- phosphoinositide

PKC- protein kinase $C$

PLC- phospholipase

PMA- phorbol myristate acetate

PPRs- pattern recognition receptors

PX domain- Phagocyte oxidase homology domain

qRT-PCR- quantitative real-time polymerase chain reaction

Rac- Ras-related C3 botulinum toxin substrate

RBCs- red blood cells 
RILP- Rab7 interacting lysosomal protein

RME- receptor mediated endocytosis

ROI- reactive oxygen intermediate

ROS- reactive oxygen species

SDS-PAGE- sodium dodecyl sulfate polyacrylamide gel electrophoresis

siRNA- small interfering RNA

SNARE- soluble NSF attachment protein receptors

Src- Sarcoma-family kinases

Syk- spleen tyrosine kinase

Tiam- T-cell lymphoma invasion and metastasis

TGF $\beta$ - transforming growth factor beta

TLR-Toll like receptor

TNF- tumor necrosis factor

TRPML- transient receptor potential mucolipin

V-ATPase- vacuolar $\mathrm{H}^{+}$ATPase

WASP- Wiskott-Aldrich syndrome protein 
CHAPTER 1

Introduction 


\subsection{Immune system}

Control and prevention of infectious diseases remains a critical global health problem, despite the existence of a powerful immune system that controls and eliminates the majority of potential infectious organisms. In mammalian organisms, the immune system is composed of two parts: innate and adaptive immunity (Akira, Uematsu, \& Takeuchi, 2006). The innate immune system is the host's first line of defense, responding to a limited number of antigens with fast kinetics, and initiates the inflammatory response. In contrast, the adaptive immune system recognizes a wide varity of antigens with slower kinetics (Borghesi \& Milcarek, 2007). The innate immune system is composed of different cell types such as macrophages, dendritic cells (DCs), and neutrophils, that interact with each other and create a network that defend our body. These cells possess pattern recognition receptors (PRRs) on their surface that either detect pathogen associated molecular patterns (PAMPs), such as microbial carbohydrates, nucleic acids, and lipoproteins, or recognize damaged associated molecular patterns (DAMPs), which are host molecules released from an injured cell or tissue (Newton \& Dixit, 2012).

\subsection{Cells of the innate immunity}

Immune cells such as dendritic cells, neutrophils, and macrophages are well known as professional phagocytes for their ability to sense the environment, recognize and internalize pathogens as well as damaged and dying cells (Elhelu, 1983). 


\subsubsection{Dendritic cells}

DCs are crucial for initiating and regulating primary immune response. They capture, process and present antigens on their cell surface; therefore, they are well known as professional antigen presenting cells (APCs) and have four major functions such as, activating $T$ cells, maintaining immune tolerance, sustaining immune memory and regulating B cell stimulation and function. Immature DCs are found in the bone marrow and travel throughout the body in a dormant state waiting to find and capture antigens that are presented on the surface of foreign bodies and pathogens (Wick, 2003). DCs internalize antigens through the process of phagocytosis and/or receptor mediated endocytosis. Once captured and internalized, antigens are processed and degraded either through lysosomes or via proteasomes, depending on the type of antigens being presented on the cell surface. For Major Histocompatibility Complex (MHC) class I, the antigens are degraded via proteasomes, which then enter the endoplasmic reticulum where the newly synthesized class I MHC molecules bind to the peptides and are delivered to the cell surface for presentation (Ackerman \& Cresswell, 2003).

On the other hand, class II MHC uses a different pathway, in which antigens are degraded in lysosomes to form peptides that then bind to MHC class II proteins. The peptide-MHC-II complex is then trafficked to the cell surface for presentation to and activation of T cells. In addition, DCs produce different type of cytokines that induce differentiation and activation of B cells. Moreover, DCs play an important role in maintaining immune tolerance in which the immune system fails to respond to specific antigens and they do this by inducing apoptosis in T cells that express immunity against 
self (Wick, 2003). In contrast to dendritic cells, macrophages and neutrophils take immediate action upon infection and play an essential role in our innate immune defence, subsequently recruiting other immune cells to the site of infection (K. P. J. M. Van Gisbergen, Geijtenbeek, \& Van Kooyk, 2005).

\subsubsection{Neutrophils}

Neutrophils are made in bone marrow and circulate in the blood. They are the most common type of white blood cell (WBC) and make up $75 \%$ of all WBCs in the body. Neutrophils have a half-life of about four to ten hours when they are in resting state. Upon infection, neutrophils are the first type of immune cells that are recruited to the site of infection by chemical cues through the process of chemotaxis (Palmer, Diehn, Alizadeh, \& Brown, 2006). Neutrophils are well known as professional phagocytes, which mean they ingest pathogens through the process of phagocytosis and form phagosomes. In some cases, before ingestion of invading pathogens, neutrophils release a net of fibers known as neutrophil extracellular traps (NET) that trap pathogens, killing and degrade them outside of the cells. Once the pathogen is trapped, whether through phagocytosis or via NETs, primary, secondary, and tertiary granules, which contain antimicrobial and cytotoxic substances, are then delivered to the exterior of the cell or to the phagosome, releasing their content and degrading the invading pathogen (Faurschou \& Borregaard, 2003). 


\subsubsection{Macrophages}

Macrophages are mononuclear white blood cells known as professional phagocytes. The word macrophage means "big eater" (Madsen, Baek, Makkouk, Krasieva, \& Hirschberg, 2012). They are involved in detection, internalization and destruction of pathogens, dying cells and debris in our bodies. Monocytes, which makes $10 \%$ of total leukocytes in human and $2-4 \%$ in mice, are produced by stem cells in the bone marrow and are released in to the blood stream where they remain and circulate in the blood for 1-2 days. If they have not been recruited to a tissue to fight a danger, they die and are removed (Italiani \& Boraschi, 2014). However, if they reach a tissue, they differentiate and mature into macrophages, where they live for months and patrol and protect the organs and cells. Thus, macrophages are divided into two subpopulations, tissueresident macrophages and monocyte-derived macrophages (Italiani \& Boraschi, 2014).

The tissue resident macrophages are produced before the hematopoietic stem cells precursors exist in embryo and these macrophages are generated straight from the yolk sac of the embryo or the liver of the fetus, which then travel to various tissues and adapt to that microenvironment. For example, Kupffer cells reside in the liver and are responsible for phagocytosis of red blood cells, microglia are found in the central nervous system, and alveolar macrophages are located in the lungs (Gordon, 2003). One of the unique characteristics of tissue resident macrophages is that some of them are capable of regenerating and dividing to form new macrophages, while others can be supplied by monocytes from the blood stream (Davies, Jenkins, Allen, \& Taylor, 2013). In addition to fighting and clearance of pathogens, tissue resident macrophages are also 
involved in maintaining tissue homeostasis by removing dying cells, debris, and toxic materials. Importantly, tissue resident macrophages suppress inflammation, restoring tissue homeostasis following infection or injury. In most circumstances, the population of these resident macrophages are insufficient to fight infections; therefore, they receive help from monocyte derived macrophages from the blood stream and differentiate into macrophages to help clear infection (Murray \& Wynn, 2011). In comparison, monocyte-derived macrophages are inflammatory macrophages that are recruited to the site of infection by tissue resident macrophages. Studies have shown that not all monocyte derived macrophages die during infection, but some differentiate and become tissue resident macrophages. The inflammatory monocyte derived macrophages are antigen presenting cells, inducing polarization of Th1 cells (Italiani \& Boraschi, 2014).

In addition to pathogen killing and phagocytosis, macrophages are also capable of releasing cytokines, small proteins $(5-20 \mathrm{kDa})$ that act as a chemical messenger and regulates the function of other cells, simply exploit as a communication tool between cells of the immune system and control the body's response to infection and diseases. There are different types of cytokines such as interferons, lymphokines, chemokines, interleukins, and tumor necrosis factor, which work together and help regulate the immune response (J.-M. Zhang \& An, 2007). Cytokines are divided into two subpopulations of pro-inflammatory and anti-inflammatory. As the name implies, proinflammatory cytokines such as interleukin-1 (IL-1), IL-12, IL-18, tumor necrosis factor $\alpha(\mathrm{TNF} \propto)$, and $\delta$ interferon (INF- $\delta$ ) induce inflammation, leading to activation and 
recruitment of other immune cells to the site of infection (Newton \& Dixit, 2012). In contrast, anti-inflammatory cytokines such as IL-10, IL-4, IL-13, IL_23, transforming growth factor $\beta$ (TGF $\beta$ ), and INF- $\propto$ induce healing, suppress inflammatory signals and preclude over activation of immune response by regulating generation and activation of $\mathrm{T}$ and $\mathrm{B}$ cells and subsequently prevention of autoimmune disease (Su, Lu, Shen, Li, \& Sun, 2012).

In addition, cytokines govern the differentiation and function of macrophages. Monocytes can be differentiated into pro or anti-inflammatory macrophages based on the presence of a specific cytokines/signal and are classified as M1 or M2 respectively (Makita, Hizukuri, Yamashiro, \& Murakawa, 2014). The pro-inflammatory macrophages, M1, are induced by Toll Like Receptor (TLR) agonists and INF $\gamma$, produce and secrete high level of pro-inflammatory cytokines such as TNF $\alpha, \mathrm{IL}-12, \mathrm{IL}-6, \mathrm{IL}-1 \beta$ and IL-23 as well as production of nitric oxide synthase (NOS2) and reactive oxygen intermediate (ROI). M1 macrophages induce Th1 polarization of CD4 lymphocytes and comprise the first line of defense upon presence of intercellular pathogens (Muraille, Leo, \& Moser, 2014). M2 polarized macrophages produce a high level of IL-10 and TGF- $\beta$, induce Th2 polarization, suppress inflammation, and take part in tissue remodeling (Italiani \& Boraschi, 2014).

\subsection{Mechanisms of internalization by immune cells}

Cells of innate immunity internalize a diverse set of cargo molecules such as nutrients, fluid, lipids, membrane proteins, and cell debris through the processes of endocytosis. Endocytosis comes from the Greek roots; endon means within, kytos means cell, and 
osis means process (Barker, 1968). Endocytosis is a process by which substances are brought into the cell without passing through the cell membrane and is energy dependent. The internalized cargo molecules are either recycled back to the surface of the cell via early endosomes or delivered to the lysosomes through the endocytic pathway for degradation purposes. The endocytic pathway is composed of three main endosomal compartments; early endosomes, late endosomes, and lysosomes. Soon after internalization, the endocytic vesicle fuses with early endosomes which then mature into late endosomes, and over time late endosomes are converted into lysosomes, which are highly acidic and contain hydrolytic enzymes (Huotari \& Helenius, 2011). There are three different types of endocytosis: pinocytosis, receptor-mediated endocytosis, and phagocytosis (Mellman, 1996).

\subsubsection{Pinocytosis}

Pinocytosis, or fluid phase endocytosis, is the process by which a cell takes in fluids and small molecules $(\leq 100 \mathrm{~nm})$ that are dissolved in the fluid. During the process of pinocytosis, the cell membrane folds around itself and creates a small pocket around the fluid, which closes to form a vesicle. At this stage, any small molecules and liquid that are trapped inside the vesicle are taken into the cell (Alberts et al., 2002).

\subsubsection{Receptor-mediated endocytosis}

The major difference between pinocytosis and receptor-mediated endocytosis (RME) is that the in-folding of the membrane around small molecules occurs only when the 
molecule binds directly to a receptor on the surface of the cells. Binding of the target molecules to the receptor triggers a signaling cascade, which leads to in-folding of the membrane around the molecule and closure of the membrane to form a vesicle. Only specific cargo molecules can be internalized through RME, which is highly dependent on receptor-ligand interaction.

\subsubsection{Phagocytosis}

Phagocytosis is a complex, active, and receptor mediated internalization of solid particles ( $\geq 500 \mathrm{~nm}$ ) by phagocytes, which have receptors on their surface that recognize and bind the target particle. Binding of the particle triggers a signal that leads to cytoskeleton rearrangement and formation of the phagocytic cup around the particle. Eventually, the solid particle is engulfed and sequestered into a phagosome. The newly formed phagosome then fuses with early endosomes and finally with lysosomes to form phagolysosomes. This process is known as phagosome maturation and involves a coordinated series of membrane fusion and fission events (Steinberg \& Grinstein, 2008).

There are two types of phagocytosis, inflammatory and non-inflammatory. Noninflammatory phagocytosis includes engulfment and degradation of dying cells without activation of the immune system (Teo \& Hughes, 2003). In contrast, inflammatory phagocytosis has an important role in innate immunity, which involves ingestion of pathogens by lysosomal enzymes and secretion of inflammatory substances to enhance the immune response (Aderem, 2003). 


\subsection{Lysosomes: small organelles with big responsibilities}

Lysosomes are highly acidic organelles containing more than 50 lysosomal hydrolases such as nucleases, phosphatases, glycosidases, lipases, proteases and many more, which are crucial in pathogen degradation and cellular defense. The acidic environment of lysosomes $(\mathrm{pH} 4.5-5.5)$ is maintained by $\mathrm{H}^{+}$-ATPase, which pump protons into the lumen of lysosomes. The majority of lysosomal enzymes work optimally in low pH environment (Colletti, 2011). In resting cells, lysosomes are morphologically small, round organelles (appear punctate under the microscope); however, the size and shape of lysosomes change under various conditions and give them specific characteristics. For example, treatment of macrophages with YM 201636, a selective inhibitor of PIKfyve, leads to the enlargement of lysosomes (Jefferies et al., 2008), while exposure to lipopolysaccharides (LPS) causes lysosomal branching and tubulation (Mrakovic, Kay, Furuya, Brumell, \& Botelho, 2012).

Lysosomes are not just terminal organelles of the endocytic pathway, but are dynamic organelles that are involved in different physiological process such as bone and tissue homeostasis, cholesterol homeostasis, cell signaling, membrane repair, cell death and pathogen defense (Saftig \& Klumperman, 2009). Lysosomal biogenesis is highly dependent on incorporation of the biosynthetic and endocytic pathway. Lysosomes are continuously receiving newly synthesized proteins that are made in the endoplasmic reticulum, transported to the trans-Golgi network and from there to the plasma membrane and subsequently, through the endocytic pathway to reach lysosomes. Among other pathways, mannose-6-phosphate receptor (M6PR) employs clathrin-based 
vesiculation to transport lysosomal hydrolases from the Golgi into the lysosomes (Coutinho, Prata, \& Alves, 2012; Kornfeld \& Mellman, 1989).

In addition to lysosomal hydrolases, integral lysosomal membrane proteins (LMPs) are also delivered to lysosomes. More than 25 different LMPs have been identified, which have diverse functions such as protein import from the cytosol, export of degradation products to the cytosol, membrane fusion, and acidification of the lysosomes (Eskelinen, Tanaka, \& Saftig, 2003). Two of the best known LMPs are lysosomal associated membrane protein I and II (LAMPI and II), which have a high degree of similarity. LAMPI and II have a short C terminal domain and a highly glycosylated luminal domain, which form a shield and protect lysosomal membrane proteins from digestion by its own proteases (Cuervo \& Dice, 2000). Moreover, lysosomes were found to be dispersed and more peripherally localized in LAMPs deficient cells, which indicates the important role of LAMP in lysosomal dynamics and migration toward the microtubule organizing center. LAMPs also regulate lysosomal exocytosis, especially during plasma membrane repair. In addition, the C terminal of LAMP II is implicated in chaperone mediated autophagy (Saftig \& Klumperman, 2009).

Despite being known as the "garbage disposal of the cell", lysosomes are also the center of the two major outgoing routes of membrane trafficking, namely, retrograde trafficking pathway from lysosomes to Golgi, and exocytosis, from lysosomes to plasma membrane. In retrograde trafficking, degraded and recycled materials are packaged into vesicles, bud off from lysosomes and are delivered to the trans-Golgi network, which can be used for the synthesis of new lipids and proteins. The other outgoing route, 
exocytosis, involves the delivery of membrane and/or materials to the plasma membrane and extracellular environment. Exocytosis, a fusion event between lysosomal membrane and plasma membrane, is calcium dependent. This calcium is released from lysosomal stores by opening of the lysosomal calcium channel, TRPML1 (Paul R. Pryor, Mullock, Bright, Gray, \& Luzio, 2000). Recently, studies have shown that lysosomes play a crucial role for particle uptake during phagocytosis of large particles (> $5 \mu \mathrm{m}$ ) or internalization of multiple particles by a single phagocyte. They serve as a major source of intercellular membrane in which they deliver lysosomal membrane and proteins to the cell surface through fusion with the plasma membrane (M. Samie et al., 2013).

\subsection{Phagocytosis and phagosome maturation in macrophages}

Being well known as professional phagocytes, the primary function of macrophages is phagocytosis. Solid particles are engulfed by macrophages and sequestered into a phagosome. Phagosomes then fuse with early and late endosomes and ultimately with lysosomes to mature into phagolysosomes. This process is known as phagosome maturation, and the formation of highly acidic and degradative phagolysosomes play an important role in degradation of internalized particles (Steinberg \& Grinstein, 2008).

Phagocytosis begins when associated ligands on a particle bind to specific receptors on the phagocyte membrane. Macrophages have different types of receptors, which help them recognize, bind and internalize a large group of pathogens. For example, altered proteins, sugars, and lipids on the apoptotic cell surface is recognized by scavenger receptors, lipopolysaccharide by CD14 receptor, sugar residues present on 
the surface of microorganisms by lectin receptors. The most well-studied phagocytic receptor is the Fcy receptor, which recognize and bind to the $\mathrm{Fc}$ portion of Immunoglobulins (Ig), an opsonin that coats the target particle (Garcl, 2002).

Binding of IgG-coated particles triggers tyrosine phosphorylation of Fcy receptor and associated proteins, which induce clustering of FcyR at the particle contact site and increase avidity of binding that leads to initiation of signal transduction required for the formation of phagocytic cup and efficient particle uptake (Sobota et al., 2005). Phosphorylation of the tyrosine residues on the ITAM (immunoreceptor tyrosine based activation motif), a special amino acid motif located in the cytosolic tail of FcyRs, by Src tyrosine kinase serves as a docking site for the Syk tyrosine kinase. Once Syk is recruited to the plasma membrane and bound to the ITAM motif, it is activated and stimulates other proteins that induce actin polymerization, membrane protrusion and formation of the phagocytic cup (Figure 1.1). 


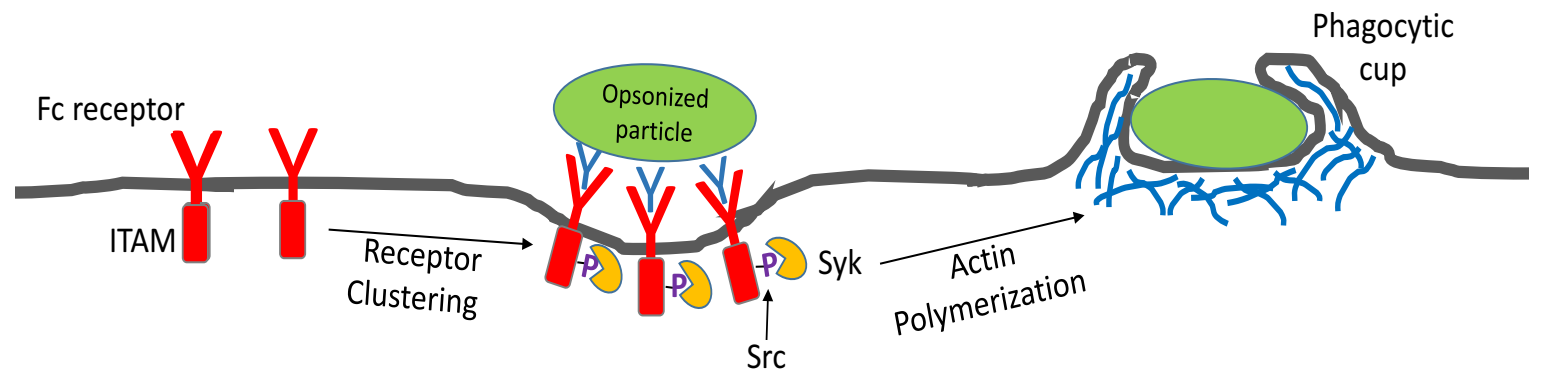

Figure 1.1: Fcyreceptor mediated phagocytosis in macrophages.

IgG-coated (opsonized) particle binds to Fc $\gamma$ receptors and upon binding initiates a signaling cascade that leads to the lateral movement and clustering of the receptors at the site of phagocytosis. Binding of the particle recruits Src kinase, which phosphorylates the cytosolic domain of the Fc receptor, ITAM. Phosphorylation of ITAM serves as a docking site for Syk kinase, which causes activation of Syk. Activated Syk acts on other proteins that are involved in actin polymerization and formation of the phagocytic cup around the particle being internalized. Information for this drawing was taken from Figure 4 in (Garcl, 2002).

Phosphatidylinositol 3-kinase (PI-3K), a lipid kinase that phosphorylates the inositol ring of phosphatidylinositol at position 3 and its lipid products phosphatidylinositol-3,4 bisphosphate $\left[\mathrm{PI}(3,4) \mathrm{P}_{2}\right]$ and phosphatidylinositol-3,4,5 triphosphates $\left[\mathrm{PI}(3,4,5) \mathrm{P}_{3}\right]$ are found at the site of phagosome formation. In addition to $\mathrm{PI}(3,4,5) \mathrm{P}_{3}$, phosphatidylinositol-4,5 bisphosphate $\left[\mathrm{PI}(4,5) \mathrm{P}_{2}\right]$ is also present at the site of phagocytosis. $\mathrm{PI}(4,5) \mathrm{P}_{2}$ is produced by the enzyme $\mathrm{PI}(4) \mathrm{P}$ 5-kinase, a lipid kinase that phosphorylates $\mathrm{PI}(4) \mathrm{P}$ in position 5 and generates $\mathrm{PI}(4,5) \mathrm{P}_{2}$, and is known to affect different actin binding proteins as well as controlling actin assembly by actin related protein 2 and $3(\operatorname{Arp} 2 / 3)$ complex though Wiskott-Aldrich syndrome protein (WASP) (Rohatgi, Ho, \& Kirschner, 2000). In addition to $\mathrm{PI}(4,5) \mathrm{P}_{2}$, members of Rho family 
GTPases, Cdc42, Rac1, and Rac2 also control actin filament dynamics by stimulating WASP, which in turn stimulates ARP $2 / 3$ to nucleate actin branching at the site of phagocytosis, leading to pseudopod extension and formation of phagocytic cup around the particle to be internalized (Carlier, Ducruix, \& Pantaloni, 1999). In addition, PI-3K activates myosin light chain kinase (MLCK), which stimulates myosin. ATP hydrolysis by myosin generates mechanical force that helps extend membrane pseudopods around the particle (Garcı, 2002). Membrane protrusion is followed by sealing of the phagocytic cup, also facilitated by $\mathrm{PI}-3 \mathrm{~K}$ by recruiting amphiphysin II to the phagocytic cup, a protein that plays an important role in recruiting dynamin-2 (Gold et al., 2000), a GTPase that is involved in membrane scission. Dynamin-2 either directly generates the mechanical force for vesicle scission, or indirectly activates other effector proteins to accomplish the scission of the phagosome from the plasma membrane (Garcl, 2002), though phagosome scission remains poorly understood.

Shortly after sealing, the newly formed phagosome travels inside the cell and fuses with the endocytic compartments such as early endosomes, late endosomes, and finally lysosomes to mature into phagolysosomes. Regulation of phagosome-lysosome fusion is very important because some pathogens like Mycobacterium tuberculosis (Mtb) are capable of escaping phagosome maturation and reproduce within the host macrophage. For example, M. tuberculosis has evolved two strategies to avert degradation by macrophages. They produce phosphatidylinositol analogs such as lipoarabinomannan (LAM), which prevent phagosomal acquisition of late endosome/lysosome constituents (Vergne, Chua, \& Deretic, 2003). They also produce 
phosphatidylinositol mannoside (PIM), which facilitates fusion of phagosomes with early endosomes that delivers nutrients such as iron to aid intracellular survival of $M$. tuberculosis (Vergne et al., 2004).

During the process of maturation, the phagosome acquires different proteins and lipids, that facilitates phagosome fusion with endocytic compartments. For example, one of the first steps in phagosome maturation is the fusion of the phagosome with early endosomes. This process is highly dependent on the presence of $\mathrm{PI}(3) \mathrm{P}$ on the phagosomes. For example, EEA1 is recruited to membranes by binding $\mathrm{PI}(3) \mathrm{P}$ though the EEA1 FYVE domain and to Rab5 GTPase. EEA1 tethers and bridges endosomes to other endosomes or phagosomes to mediate fusion (Otilia V. Vieira et al., 2001). Fusion of the phagosome with early endosomes changes the $\mathrm{pH}$ of the phagosome and makes it more acidic by acquisition of the V-ATPase proton pump from early endosomes. Phagosomal acquisition of Rab5 is transient and is followed by recruitment of Mon1-Ccz1 complex, a Rab7-GEF, on the surface of the phagosome. Mon1-Ccz1 binds directly to Rab5-GTP and converts Rab7-GDP to Rab7-GTP (Nordmann et al., 2010). The transition from Rab5 to Rab7 is followed by further acidification of the phagosomes through fusion of phagosome with late endosomes and acquisition of additional V-ATPase proton pump (Figure 1.2). Rab7 interacting lysosomal protein (RILP) is an effector of Rab7 that binds to the Rab7-positive phagosomes and tethers organelles to dynein-dynactin to facilitate transportation of the phagosome via microtubules towards lysosomes (Harrison, Bucci, Vieira, Schroer, \& Grinstein, 2003). Upon fusion with lysosomes, phagosomes become highly acidic, obtain all the necessary enzymes required for the degradation of 
internalized particles (Figure 1.2), and acquires lysosomal associated membrane protein1 and 2 (LAMP I and II), (Kinchen \& Ravichandran, 2008).

LAMPI and II are required for phagosome lysosome fusion. Phagosomes deficient for both LAMPI and LAMPII acquired Rab5 but fail to recruit Rab7 and fuse with lysosomes (Huynh et al., 2007).

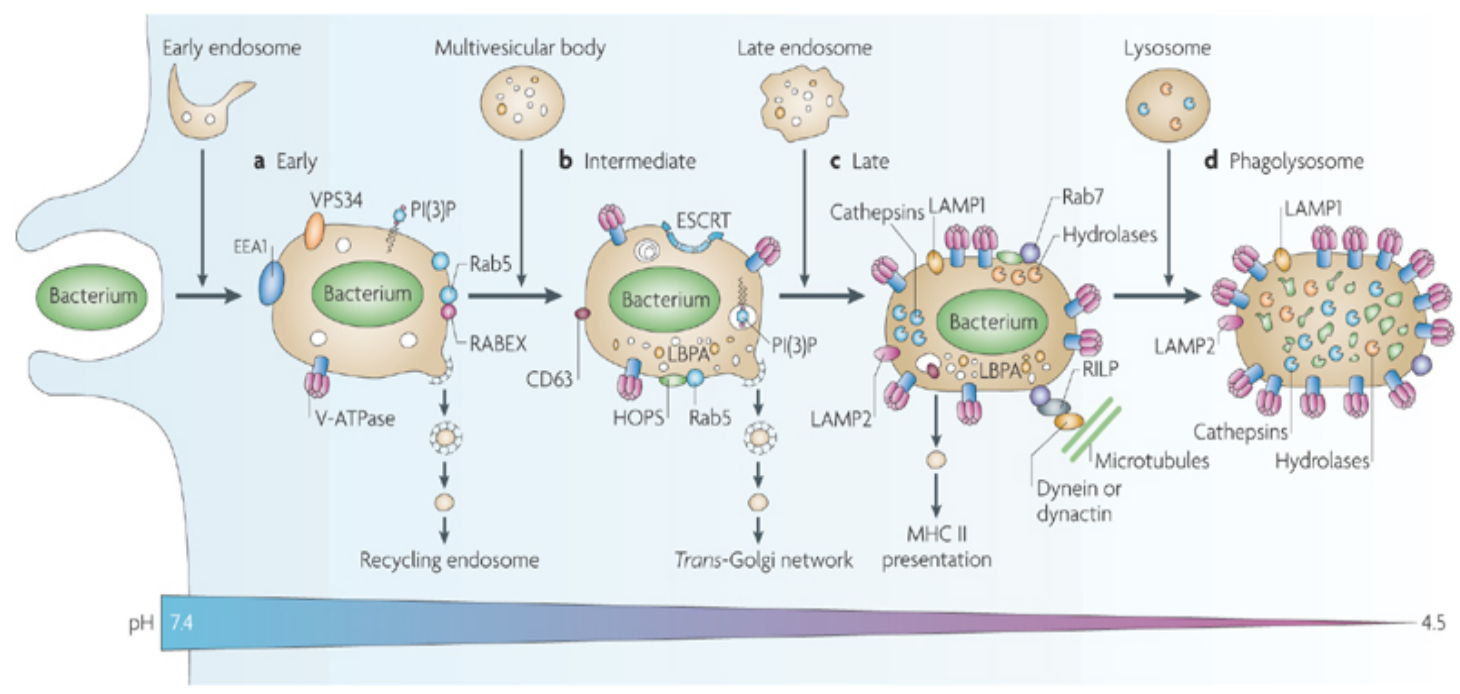

\section{Figure 1.2: The process of phagosome maturation in macrophages}

The internalized particle, in this case a bacterium, goes through the process of phagosome maturation by fusing with early and late endosomes and finally lysosomes to mature into a hybrid organelle called phagolysosome. Fusion of phagosomes with the endocytic compartments are highly dependent on certain proteins and lipids that decorate these organelles. Early phagosomes are enriched with PI3P, Rab5 and EEA1 and have a $\mathrm{pH}$ of 6 . As the phagosome fuses with late endosomes and lysosomes the $\mathrm{pH}$ drops to 4.5 and it acquires lysosomal markers such as Rab-7, LAMPI, LAMPII, PI3,5P2, VATPase, and different lysosomal enzymes such as hydrolase and cathepsins. Figure from (Flannagan, Cosío, \& Grinstein, 2009). 


\subsection{Phosphoinositides: Important spatio-temporal regulators of cell signaling \& intracellular trafficking}

Phosphoinositides (PIs) are signaling molecules that are composed of a glycerol back bone, two fatty acid chains, and a cyclic polyol myo-inositol head group. Pls are synthesized in the endoplasmic reticulum (ER) from myo-inositol and CDP-DAG with the help of phosphatidylinositol synthase (PIS) enzyme. The lipid is then distributed in the cell via vesicular trafficking and several phosphatidylinositol transfer proteins (PITPs) (Balla, 2013). The inositol head group contains hydroxyl groups that can be phosphorylated in three positions by cytosolic lipid kinases and form seven different PI species (Figure 1.3) (Falkenburger, Jensen, Dickson, Suh, \& Hille, 2010). These lipids recruit a unique set of effector proteins, which have PI recognition domains. Upon binding, the effector proteins are either guided to the appropriate location inside the cell or their activity is being modulated (Lietha, 2011). 


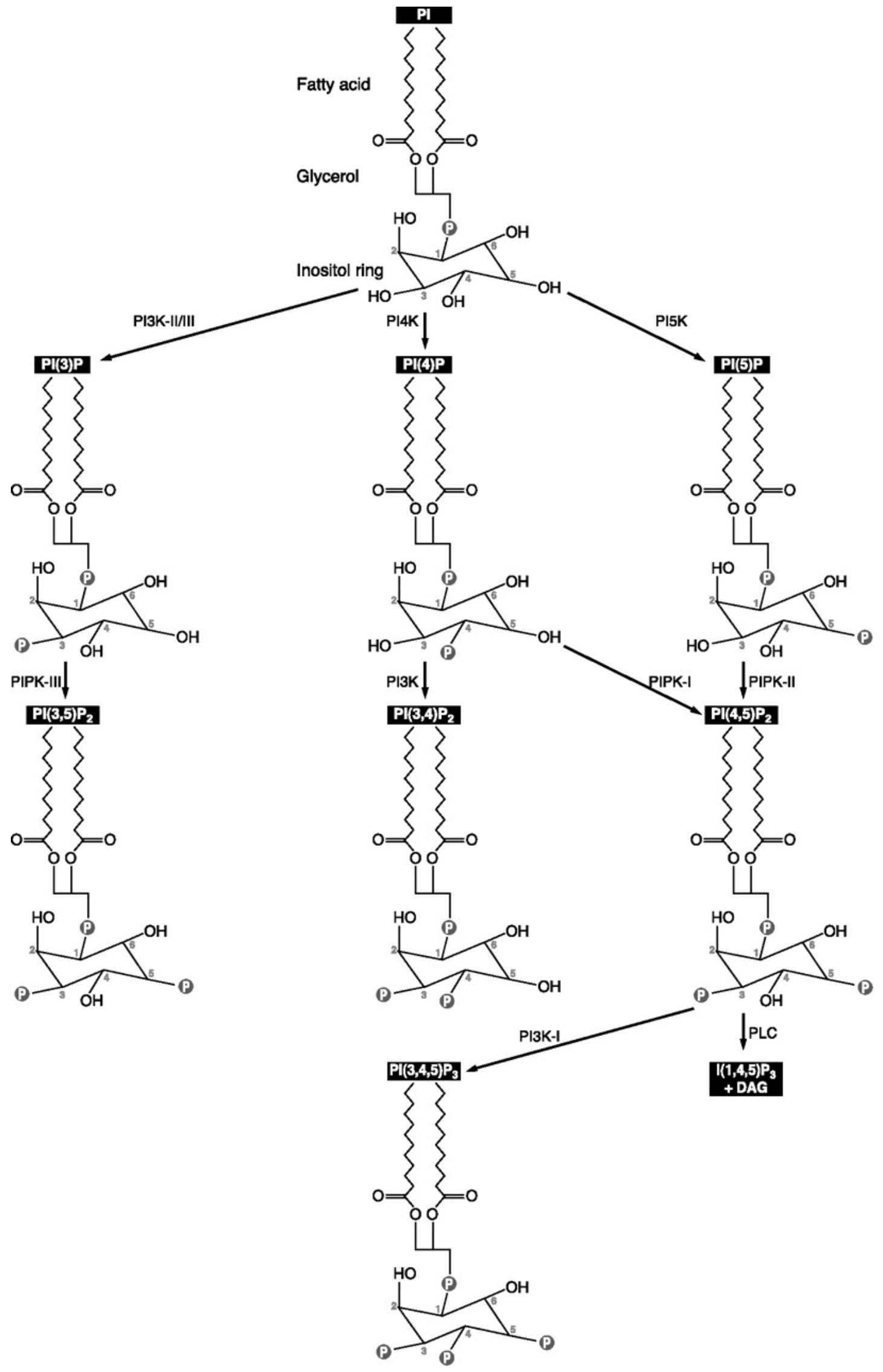




\section{Figure 1.3: Phosphatidylinositol and its seven phosphorylated species.}

With the help of different kinases and phosphatases, phosphatidylinositol can be phosphorylated or dephosphorylated at positions 3, 4 or 5 of the inositol ring and produce seven distinctive species; $\mathrm{PI3p}, \mathrm{PI} \mathrm{P}, \mathrm{PI} \mathrm{P}, \mathrm{PI}(3,5) \mathrm{P}_{2}, \mathrm{PI}(3,4) \mathrm{P}_{2}, \mathrm{PI}(4,5) \mathrm{P}_{2}$, and $\mathrm{PI}(3,4,5) \mathrm{P}_{3}$. Image was obtained from (Saarikangas, Zhao, \& Lappalainen, 2010).

In a study done by Lemmon et al., beads and liposomes, which were decorated with phosphatidylinositol-3,5-bisphosphate $\quad\left[\mathrm{PI}(3,5) \mathrm{P}_{2}\right]$ and phosphatidylinositol-4,5bisphosphate $\left[\mathrm{PI}(4,5) \mathrm{P}_{2}\right]$, were isolated and the bound proteins to these lipids were identified by mass spectrometry. A total of 388 proteins were pulled down that recognized either $\mathrm{PI}(3,5) \mathrm{P}_{2}$ and/or $\mathrm{PI}(4,5) \mathrm{P}_{2}$ and these proteins contained Pleckstrin homology (PH), Phagocyte oxidase homology (PX), epsin N-terminal homology (ENTH) and C2 domains. These proteins were identified as GTPases and GTPase regulators, kinases and phosphatases, proteins involved in membrane trafficking and cargo transport, and proteins that regulate the actin cytoskeleton (Lemmon, 2008).

Pls are also involved in membrane identity. For example, $\mathrm{PI}(4,5) \mathrm{P}_{2}$ and phosphatidylinositol-3,4,5-trisphosphate $\left[\mathrm{PI}(3,4,5) \mathrm{P}_{3}\right]$ are enriched at the plasma membrane, $\mathrm{PI}(4) \mathrm{P}$ is associated with secretory vesicles and trans-Golgi, $\mathrm{PI}(3,4) \mathrm{P}_{2}$ is associated with early endosome, $\mathrm{PI}(3,5) \mathrm{P}_{2}$ enriched in late endosomes/lysosomes, and phosphatidylinositol-3-phosphate $[\mathrm{PI}(3) \mathrm{P}]$ associates multi vesicular bodies and endosomes (Falkenburger et al., 2010).

In addition, $\mathrm{PI}(3) \mathrm{P}$ plays an important role in phagosome maturation by it recruiting early endosome autoantigen (EEA1) to the surface of newly formed phagosomes. $\mathrm{PI}(3) \mathrm{P}$ also serves as a precursor for the synthesis of phosphatidylinositol-3,5-bisphosphate 
$\left(\mathrm{PI}(3,5) \mathrm{P}_{2}\right)$ with the help of PIKfyve, a phosphatidylinositol-3-phosphate 5-kinase that binds to $\mathrm{PI}(3) \mathrm{P}$ via its FYVE domain and phosphorylates the D-5 position in $\mathrm{PI}(3) \mathrm{P}$.

\subsubsection{PIKfyve, a FYVE finger-containing phosphoinositide kinase}

PIKfyve plays an important role in cell viability since complete loss of PIKfyve leads to developmental defects and embryonic lethality (Ikonomov et al., 2011). PIKfyve has also been reported to regulate multiple cellular processes in mammals such as autophagy, and endosomal trafficking. Recent studies have linked PIKfyve to immunity using apilimod, a clinically evaluated anti-inflammatory drug and also a potent and selective inhibitor of PIKfyve. They have shown that inhibition of PIKfyve interfere with expression of IL-12, control inflammation and treat autoimmune disorder (Cai et al., 2013).

PIKfyve is only active within the PIKfyve-ArPIKfyve-Sac3 (PAS) core complex (Figure 1.4). Sac3 is the phosphatidylinositol 3,5-bisphosphate 5-phosphatase, which converts $\mathrm{PI}(3,5) \mathrm{P}_{2}$ to $\mathrm{PI}(3) \mathrm{P}$, ArPIKfyve (Associated Regulator of PIKfyve) is a scaffold for PIKfyve and Sac3 complex and promote their kinase and phosphatase activity (Ognian C. Ikonomov et al., 2011). In addition to $\mathrm{PI}(3,5) \mathrm{P}_{2}$, PIKfyve also produces phosphatidylinositol 5-phosphate $[\mathrm{PI}(5) \mathrm{P}]$, another signaling molecule that plays an important role in membrane trafficking. 


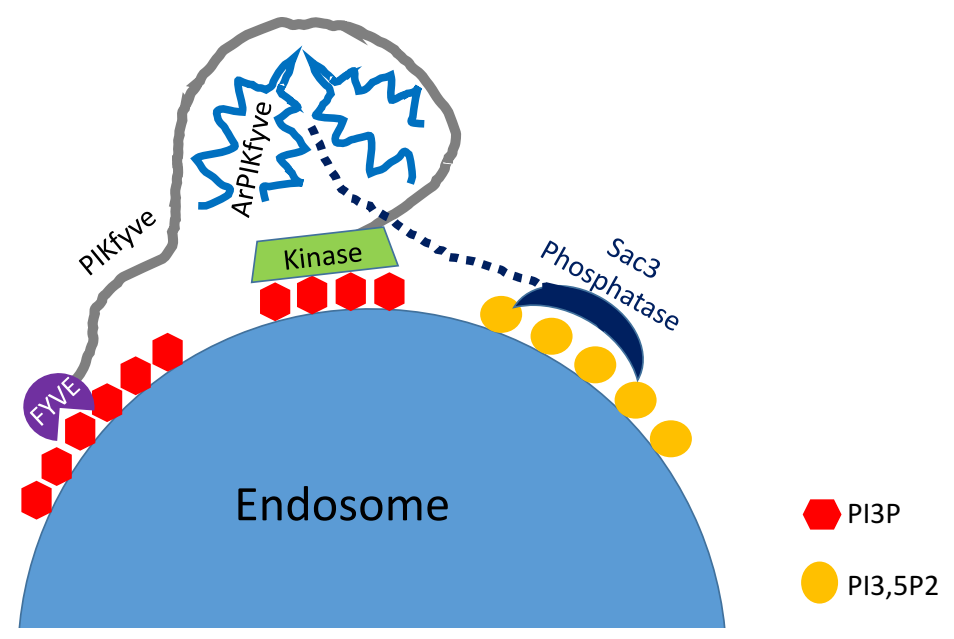

Figure 1.2: The PAS complex:

PIKfyve and Sac3 are scafolded by ArPIKfyve and form the PAS complex which is crucial for PIKfyve and Sac3, kinase and phosphates activity respectively. Information for this drawing was taken from Figure 6 in (Ognian C. Ikonomov, Sbrissa, Fenner, \& Shisheva, 2009).

$\mathrm{PI}(3,5) \mathrm{P}_{2}$ is enriched in late endosomes/lysosomes; however, a small pool may be found near or at the plasma membrane. Inhibition of $\mathrm{PI}(3,5) \mathrm{P}_{2}$ synthesis causes various neurodegenerative diseases such as Charcot Marie Tooth disease and amyotrophic lateral sclerosis, which is characterized by weakness of the muscles of hands and legs and motor neuron degeneration of the central nervous system, respectively. At the cellular level, $\mathrm{PI}(3,5) \mathrm{P}_{2}$ deficient cells exhibit enlarged lysosome phenotype as well as trafficking defect in endocytic pathway (Y. Zhang et al., 2007).

Only a few effectors are known that bind to $\mathrm{PI}(3,5) \mathrm{P}_{2}$ such as Raptor (a component of the mammalian target of rapamycin complex 1, mTORC1) and the transient receptor potential mucolipin-1 (TRPML-1) (Bridges et al., 2012; Dong, Shen, Wang, Dawson, Li, Zhang, Cheng, Zhang, Weisman, Delling, $\mathrm{Xu}$, et al., 2010). $\mathrm{PI}(3,5) \mathrm{P}_{2}$ binds to the WD-40 domain of raptor, a component of the MTORC1 that regulates the metabolic state of the cell. In adipocytes, $\mathrm{PI}(3,5) \mathrm{P}_{2}$ contributes to recruitment and localization of mTORC1 to 
the plasma membrane (Bridges et al., 2012). Further evidence for a potential role for $\mathrm{PI}(3,5) \mathrm{P}_{2}$ at the plasma membrane comes from its binding to class II formins, which leads to remodeling of the cortical actin array (P. A. C. van Gisbergen, Li, Wu, \& Bezanilla, 2012). In addition, $\mathrm{PI}(3,5) \mathrm{P}_{2}$ is also known to be essential for several pathways in the endomembrane system such as retrograde trafficking of proteins from endosomes to trans-Golgi network (Rutherford et al., 2006), and formation of multivesicular body and protein degradation (Gary, Wurmser, Bonangelino, Weisman, \& Emr, 1998). In addition, $\mathrm{PI}(3,5) \mathrm{P}_{2}$ also binds and regulates activity of TRPML-1, a Ca ${ }^{2+}$ channel that is localized in the membrane of the lysosomes and allows release of $\mathrm{Ca}^{2+}$ from the lumen of the lysosomes into the cytosol, which regulates membrane trafficking pathways. For example, an increase in cytosolic $\mathrm{Ca}^{2+}$ is necessary for lysosomephagosome fusion, lysosome-lysosome fusion, and lysosome-plasma membrane fusion (Paul R Pryor, Mullock, Bright, Gray, \& Luzio, 2000). TRPML-1 is gated by PI(3,5)P2, which binds directly to the $\mathrm{N}$ terminus of this channel (Dong, Shen, Wang, Dawson, Li, Zhang, Cheng, Zhang, Weisman, Delling, Xu, et al., 2010).

\subsection{The lysosomal calcium channel, TRPML1}

TRPML channels, the mucolipin family of the transient receptor potential (TRP) super family, consist of six transmembrane domains and cytosolic amino and carboxy terminal tails (Figure 1.5). TRPML channels are non-selective cation channels that are permeable

to $\mathrm{Zn}^{2+}, \mathrm{Na}^{+}, \mathrm{Fe}^{2+}$, an $\mathrm{Ca}^{2+}$ and are activated by $\mathrm{PI}(3,5) \mathrm{P}_{2}$ (Venkatachalam, Wong, \& Zhu, 2014). There are three TRPML channels in mammals, TRPML1-3, with different 
intracellular localization. TRPML1, is localized to late endosomes and lysosomes, TRPML2 is localized to late endosomes as well as recycling endosomes (Karacsonyi, San Miguel, \& Puertollano, 2007), and TRPML3 is localized to early endosomes, late endosomes, and plasma membrane (H. J. Kim, Soyombo, Tjon-Kon-Sang, So, \& Muallem, 2009).

Mutations in the human TRPML1 gene causes type IV mucolipidosis (ML4), a pediatric neurodegenerative disease characterized by retinal degeneration, psychomotor defect, and mental retardation. At the cellular level, enlargement of late endosomes/lysosomes, abnormal lipid accumulation in the lysosomes (Treusch et al., 2004), and defects in retrograde lipid transport are seen in TRPML1 knockout mice (Paul R Pryor, Reimann, Gribble, \& Luzio, 2006). Loss of TRPML1 results in accumulation of late endosomes and lysosomes and elevation of autophagosome biogenesis. The lysosomal free amino acids, derived from proteolysis after lysosomal degradation, activate Mechanistic Target of Rapamycin kinase containing Complex-1 (MTORC1), which in turn uses these free amino acids for protein translation and cell growth (Wong, Li, Montell, \& Venkatachalam, 2012; Zoncu et al., 2011). Therefore, loss of TRPML1, which leads to decrease in proteolytic activity of the lysosomes and consequently cellular amino acid starvation, is accompanied by a drop in MTORC1 activity, which induces autophagy in the cells (Efeyan, Zoncu, \& Sabatini, 2012). In addition, loss of TRPML1 have been attributed to cell death since knockdown of TRPML1 results in lysosomal protease cathepsin B leakage into the cytoplasm and loss of lysosomal 
integrity. High level of cathepsin B in the cytoplasm initiates a signal that induces apoptosis (Colletti et al., 2012).

TRPML1 also serves as a $\mathrm{H}^{+}$leak channel that prevents over acidification of the lysosomes. However, when further acidification of the lysosomes is required for their proteolytic activity and activation of proteases and lipases, TRPML1 is cleaved and becomes inactive to restore the optimal $\mathrm{pH}$ in the lysosomes (Figure 1.5). The proteolytic cleavage of the TRPML1 is one of the interesting aspects of this channel and since the full length of the channel is the only active form, cleavage of the channel is a major form of regulating the activity of TRPML1 (Soyombo et al., 2006).

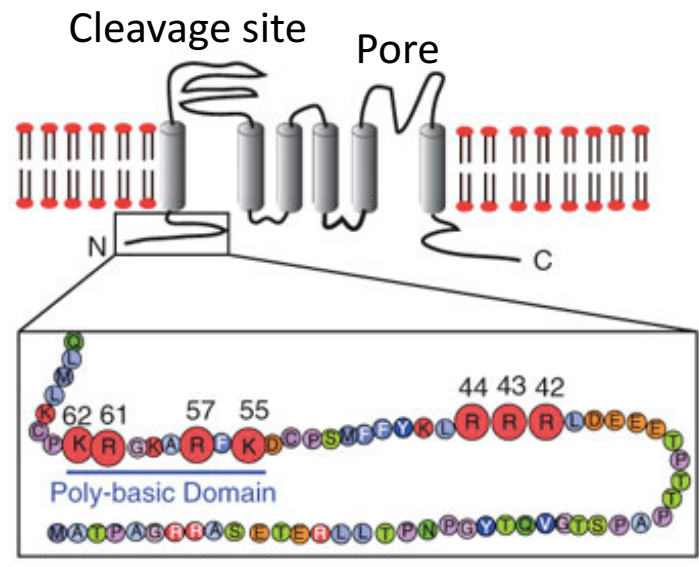

\section{Figure 1.5: Transient Receptor Potential Mucolipin-1 (TRPML-1) structure}

TRPML1 has 6 transmembrane domains that span the lysosomal membrane. The $\mathrm{C}$ terminal domain contains the lysosomal targeting sequence and the $\mathrm{N}$ terminal contains the poly basic domain which binds PI3,5P2. The cleavage site is facing the lumen of the lysosomes, which can be cleaved by lysosomal enzyme when acidification of the lysosomes are required. (Dong, Shen, Wang, Dawson, Li, Zhang, Cheng, Zhang, Weisman, Delling, Xu, et al., 2010)

Since TRPML1 resides in late endosomes/lysosomes and is gated by $\mathrm{PI}(3,5) \mathrm{P}_{2}$, then $\mathrm{PI}(3,5) \mathrm{P}_{2}$ may control phagosome-lysosome fusion partly by stimulating TRPML1 and diffusion of lysosomal $\mathrm{Ca}^{+2}$, which triggers phagosome-lysosome fusion since $\mathrm{Ca}^{+2}$ is known to induce membrane fusion post-docking of SNARE proteins. Therefore, 
$\mathrm{PI}(3,5) \mathrm{P}_{2}$-mediated signaling may be targeted by pathogens like Mycobacterium tuberculosis to alter phagosome maturation and facilitate pathogen take-over of host cells.

\subsection{Calcium and membrane fusion}

Membrane fusion can be simplified into three steps, tethering, docking, and bilayer fusion. Tethering requires Rab GTPase activity, which decorates compartments of the endocytic pathway (Rab5 on early endosomes and Rab7 on late endosomes/lysosomes) and recruit effector proteins that facilitate the tethering process. These effector proteins involve tethering factors such as homotypic fusion and protein sorting (HOPS), EEA1, lipid kinases and phosphatases and other sorting adaptor proteins, which results in forming a bridge and bringing the two endolysosomal membranes into close proximity (Grosshans, Ortiz, \& Novick, 2006).

Membrane tethering is followed by docking, which is the formation of transSNARE (soluble NSF attachment protein receptors) complex. Vamp7, Syntaxins 7 and 8 (lysosomal SNAREs proteins), and synaptotagmin VII are involved in the docking process, which is followed by release of calcium from the lysosomes and membrane bilayer fusion. Calcium binds to the C2 domains of the syntaptotagmin VII leading to the penetration of the $\mathrm{C} 2$ domains into the membrane bilayer and formation of the SNARE four helix bundles (trans-SNARE complex) and finally fusion of the two membranes (Figure 1.6) (Andrews, 2005; Chakrabarti, Andrade, \& Andrews, 2005). 


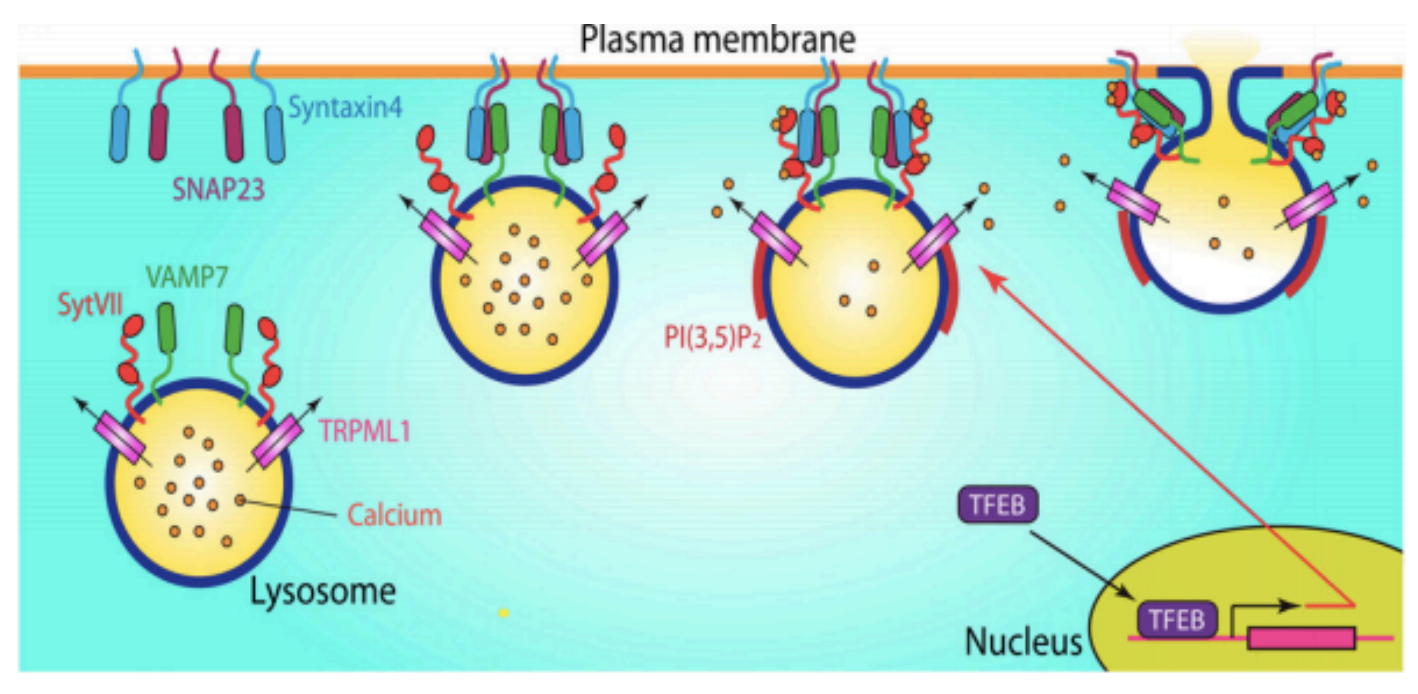

\section{Figure 1.6: The role of calcium in membrane fusion}

Calcium is being released through lysosomal calcium channel, TRPML1, which plays an important role in membrane fusion. Increase in the level of cytosolic calcium is required during membrane fusion in which calcium binds to the $\mathrm{C} 2$ domain of syntaptotagmin VII and causes insertion of the $\mathrm{C} 2$ domain into the membrane, formation of the SNARE four helix bundles, bringing the two membrane into close proximity which leads to their fusion (M. A. Samie \& Xu, 2014).

In addition to membrane fusion, calcium, as a universal second messenger, regulates other cellular processes such as proliferation, cell adhesion, and gene transcription. Therefore, the concentration of the calcium in the cytosol must be regulated and this is done through the activity of different $\mathrm{Ca}^{+2}$ exchanger, transporter, pumps, and channels. Among these, TRPML1, which is gated by $\mathrm{PI}(3,5) \mathrm{P}_{2}$, regulates the release of $\mathrm{Ca}^{+2}$ from the lysosomes in to the cytosol (Dong, Shen, Wang, Dawson, Li, Zhang, Cheng, Zhang, Weisman, Delling, Xu, et al., 2010).

\subsection{Phagocytosis and phagosome maturation in neutrophils}

In comparison to macrophages, neutrophils are extremely efficient phagocytes that take less than 20 seconds to internalize a particle (A. W. Segal, Dorling, \& Coade, 1980). 
Neutrophils express different types of receptors such as $\mathrm{Fc}_{\gamma}$ receptor, Dectin-1, Toll Like Receptor (TLR), and Nod-like receptors, which can recognize and bind invading pathogens. FcyR has different isoforms including FcyRIla, FcyRIIIb, and FcyRIV, with the former being most important for phagocytosis. Upon binding of IgG-opsonized targets, the cytosolic tail of the Fcy receptor, Immunoreceptor Tyrosine Activation Motif (ITAM), gets phosphorylated by Src family kinase at a tyrosine residue which initiates a signaling cascade that leads to actin polymerization and formation of a phagocytic cup, NADPH oxidase activation, increase in the level of cytosolic free calcium, and granules secretion (Mitchell et al., 1994). Phosphorylation of tyrosine residue at ITAM serves as a docking site for proteins with $\mathrm{Src}$ homology $2(\mathrm{SH} 2)$ domains such as the spleen tyrosine kinase (Syk), which is a tyrosine kinase. Once Syk is recruited to the site of phagocytosis and bound to the cytosolic part of the Fc receptor, it stimulates phosphatidylinositol 3 kinase (PI3K), which convert $\mathrm{PI}(4,5) \mathrm{P}_{2}$ to $\mathrm{PI}(3,4,5) \mathrm{P}_{3}$.

Syk deficient neutrophils are unable to internalize IgG coated particles (Kiefer et al., 1998). In addition to $\mathrm{PI}(3,4,5) \mathrm{P}_{3}$ synthesis, $\mathrm{PI}(4,5) \mathrm{P}_{2}$ also acts as a substrate for phospholipase C (PLC) that leads to the generation of diacylglycerol (DAG), which in turn activates protein kinase C (PKC). Activation and synthesis of these proteins and lipids induce actin polymerization and membrane remodeling, which is required for the ingestion of the particle (Lee, Harrison, \& Grinstein, 2003a). The ARP2/3 Complex, which is the major initiator of actin polymerization, is stimulated by Cell Division Control protein 42 (cdc42) and RAC through Wiskott-Aldrich Syndrome Protein (WASP) 
and SCAR/WAVE respectively. Polymerization of actin and activation of myosin $\mathrm{X}$ drives phagocytic cup formation and its sealing (Cox et al., 2002).

Shortly after sealing, the newly formed phagosome acquires cellular machineries necessary for killing and clearance of the internalized particle/microorganism through fusion of phagosomes with lysosomes and granules. In addition to early/late endosomes and lysosomes, neutrophils have specialized vesicles/granules that contain antimicrobial substances and fuse with nascent phagosomes, leading to degradation of phagosomal content (Lee et al., 2003a).

\subsubsection{Granules: lysosome-like organelles}

Neutrophils can secrete antimicrobial substances into the extracellular environment as well as into the phagosomes. This process involves different types of granules and secretory vesicles. In neutrophils, granulopoiesis is the formation of granules that occurs during myeloid cell differentiation. There are three types of granules, primary, secondary and tertiary, named based on their content and time of biosynthesis. Azurophil or primary granules are also called peroxidase-positive granules and contain a high level of myeloperoxidase (MPO), antimicrobial proteins and acidic hydrolase (Faurschou \& Borregaard, 2003). These lysosome-like organelles do not have Lysosomal Associated Membrane Protein 1 and 2 (LAMP1 and 2); however, their membrane is decorated with granulophysin (CD63). Upon release of MPO to the extracellular space or into the phagosomes (Figure 1.7), MPO reacts with $\mathrm{H}_{2} \mathrm{O}_{2}$ (a product of NADPH oxidase)

and increases the toxicity of $\mathrm{H}_{2} \mathrm{O}_{2}$. In addition, $\mathrm{H}_{2} \mathrm{O}_{2}-\mathrm{MPO}$ induces production of $\mathrm{HOCl}$ 
(hypochlorous acid), and reactive nitrogen intermediates, with all these products attacking the cell membrane of the microorganisms (Klebanoff, 1999).

In comparison, specific (secondary) and gelatinase (tertiary) granules are peroxidase-negative granules. Specific granules are larger and contain antimicrobial substances. Conversely, gelatinase granules are smaller than Azurophil granules, do not contain antimicrobial substances, and serve as a reservoir of matrix degrading enzymes. The process of degranulation, release of granule content via exocytosis, occurs upon stimulation of neutrophils with a very low concentration of N-Formylmethionyl-leucylphenylalanine (fMLP) (Sengeløv, Kjeldsen, Diamond, Springer, \& Borregaard, 1993). However, phorbol myristate acetate (PMA), a more powerful stimulant, acts specifically on each granule by inducing high levels of degranulation of tertiary granules, moderate level of degranulation of secondary granules and a small release of primary granules (Faurschou, Sørensen, Johnsen, Askaa, \& Borregaard, 2002).

\subsubsection{ROS production in neutrophils}

One of the significant features of neutrophils is the production of reactive oxygen species (ROS) such as hypochlorous acid, hydroxyl radical, hydrogen peroxide, ozone and singlet oxygen, a process known as the respiratory burst (Nordenfelt \& Tapper, 2011). Neutrophils recruit Nicotinamide Adenine Dinucleotide Phosphate (NADPH) oxidase, a multisubunit complex, to the phagosome where it allows the transfer of electrons across phagosomal membrane, which leads to the formation of superoxide in the lumen (Anthony W. Segal, 2008). The main source of phagosomal NADPH oxidase is thought to be secondary granules, which through fusion with phagosomes deliver the 
complex onto the phagosomal surface (Lee et al., 2003a). Superoxide is then converted into hypochlorous acid, which is crucial for pathogen killing (Figure 1.7). Proton consumption during superoxide production causes alkalization of phagosomes to $\mathrm{pH} 7$, which is different from that of the macrophage phagosome that has a $\mathrm{pH}$ of 5 . The $\mathrm{pH}$ of the neutrophil phagosome eventually acidifies upon fusion with lysosomes (Jankowski, Scott, \& Grinstein, 2002). The respiratory burst can be induced when cells are exposed to soluble agonists such as phorbol 12-myristate 13-acetate (PMA) or $\mathrm{N}$ Formylmethionyl-leucyl-phenylalanine (fMLP), as well as signal transduction during the process of phagocytosis, which leads to the assembly and activation of NADPH oxidase (Nordenfelt \& Tapper, 2011). Neutrophil activation by PMA is followed by assembly of oxidase components and migration of Rac2 from the cytosol onto the complex, which indicates that Rac2 behaves like a component of NADPH oxidase complex (EI Benna, Ruedi, \& Babior, 1994). In addition, the p190 Rac GTPase Activating Protein (GAP) is known to regulate NADPH activation, which indicates that the GTP form of Rac is required for oxidase activation (Heyworth, Knaus, Settleman, Curnutte, \& Bokoch, 1993). 


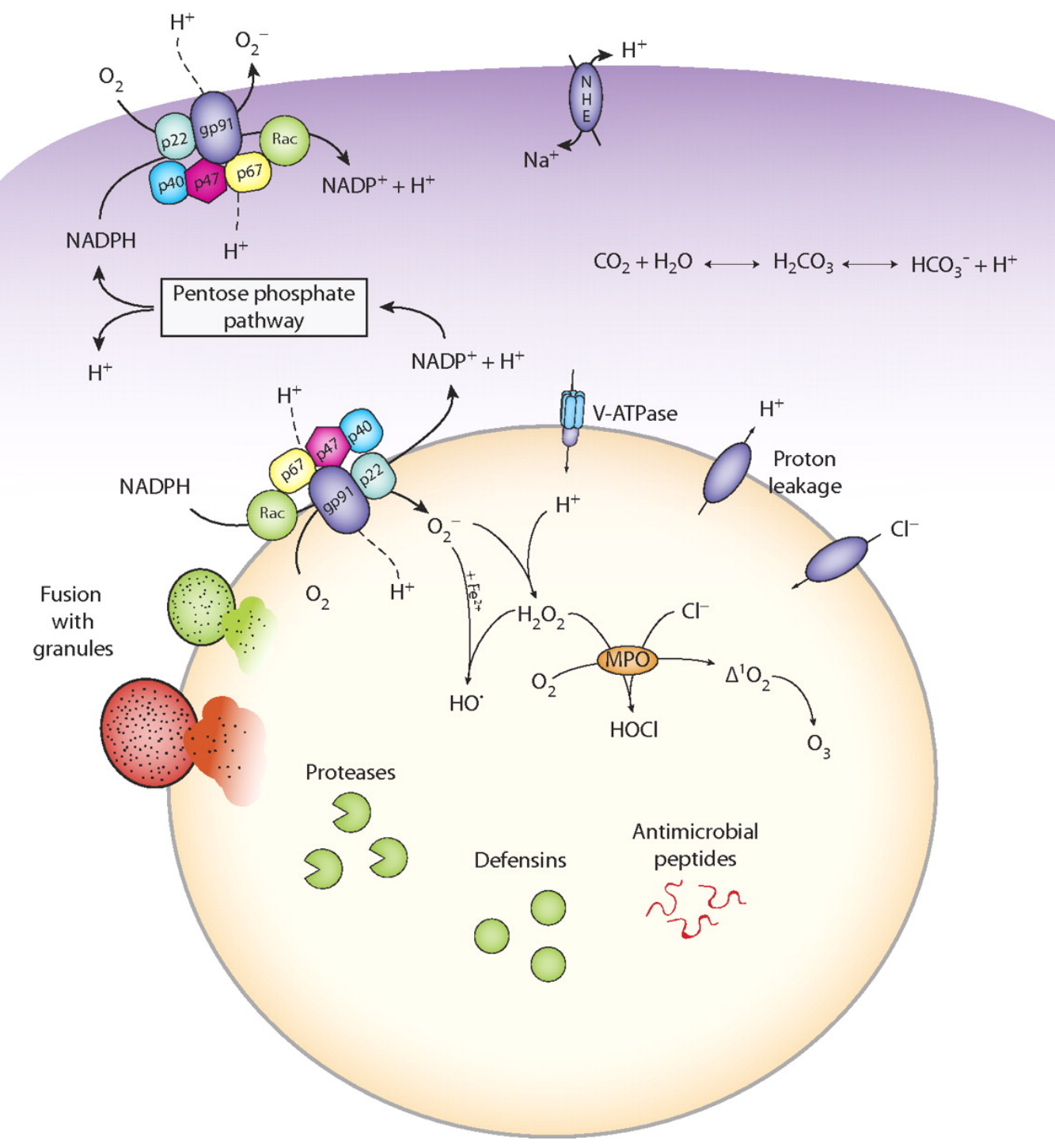

Figure 1.7: Fusion of granules with phagosome and production of reactive oxygen species: During the process of phagosome maturation, primary, secondary and tertiary granules fuse with phagosomes and deliver antimicrobial substances to the phagosome, which helps in degradation of the pathogen. Through the fusion of the secondary granules, NADPH oxidase is being delivered to the surface of the phagosome where it allows the transfer of electrons across phagosomal membrane which can be used in superoxide formation in the lumen of the phagosome (Nordenfelt \& Tapper, 2011) 


\subsubsection{RAC1 and RAC2 small GTPase during neutrophils immune responses}

RAC1 and RAC2 are members of Rho-GTPase family and are key regulators of NADPH oxidase and the actin cytoskeleton in neutrophils (Heyworth et al., 1993). There is a high degree of similarity between RAC1 and RAC2 (92\% amino acid identity), with the major difference appearing to be in the $C$ terminal region, which is involved in localization of the RAC and is the binding site for downstream effector targets. RAC2 is predominant in human neutrophils; however, the level/amount of RAC1 and RAC2 expression is similar in murine neutrophils (Glogauer et al., 2003). RAC GTPases are molecular switches and can be in one of the two conformations, active and inactive form depends on binding of GTP or GDP to the GTPase, respectively. Regulatory proteins such as Guanine Exchange Factor (GEFs) and GTPase Activating proteins (GAPs) control the cycling between GTP and GDP in RAC GTPases. GEFs exchange GDP to GTP molecules resulting in activation of RACs. Unlike GEF, GAPs hydrolyze GTP and convert RACs back into their inactive state (Figure 1.8). Active RACs recruit/bind to a large collection of effector proteins, stimulate signaling cascades and promote cellular responses such as vesicle trafficking, cell cycle progression, cytoskeletal rearrangement, and cell polarity (Xosé R. Bustelo et al, 2007). 
Figure 1.8: Rac-GTPases behave like molecular switches:

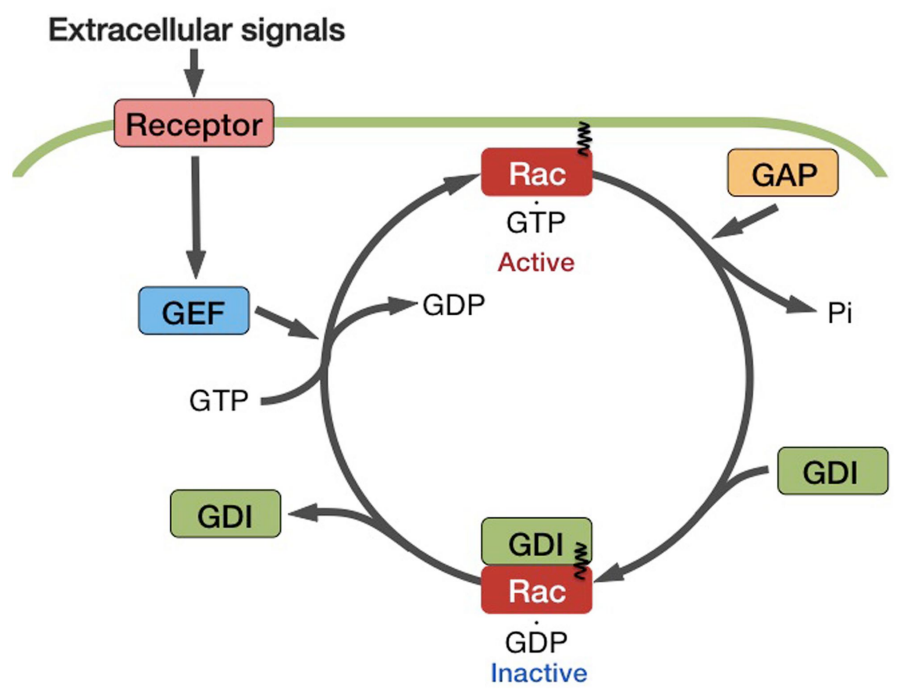

An upstream signal activates Guanine Exchange Factor (GEF), which replace GDP to GTP in Rac and turn it into its active form, which can act on a downstream effector protein. However, this molecular switch can be turned off by activating GTPase Activating Protein (GAP), which hydrolyzes the GTP and turn it into its inactive form. Image from (Kawano, Kaneko-Kawano, Shimamoto, Hückelhoven, \& Zhang, 2014)

Rac1 deletion results in embryonic lethality and its specific depletion in neutrophisl inhibits fMLP mediated actin assembly in neutrophils (Glogauer et al., 2003). Studies have shown that PI(5)P induces Rac1 activation through controlling activity of Rac-GEF known as T-cell lymphoma invasion and metastasis 1 (Tiam 1). The $\mathrm{C}$ terminal $\mathrm{PH}$ domain of Tiam1 binds to PI(5)P leading to recruitment of Rac1-GDP to dorsal ruffles as well as early endosomes, which leads to conversion of GDP to GTP by Tiam1 and activation of Rac1 (Viaud et al., 2014). This suggests a link between PIKfyve and Rac activity. 


\subsection{Research Goals}

Since the role of PIKfyve in immunity had not been explored, my first goal was to determine the role of PIKfyve in macrophage phagocytosis and phagosome maturation.

\subsubsection{Hypothesis I:}

PIKfyve inhibition interferes with phagosome and endosome maturation in macrophages

\section{Objectives:}

1. Test and use pharmacological antagonists and siRNA silencing of PIKfyve

2. Determine the role of PIKfyve/PI(3,5)P2 in phagocytosis

3. Determine the role of PIKfyve/PI(3,5)P2 in phagosome maturation

Our results concluded that pharmacological antagonists of PIKfyve and siRNA knockdown of PIKfyve interfered with phagocytosis and phagosome maturation in macrophages. Since $\mathrm{PI}(3,5) \mathrm{P}_{2}$ binds TRPML1 and regulates its activity, we then hypothesized that $\mathrm{PI}(3,5) \mathrm{P}_{2}$ helps control phagolysosome biogenesis through the activation of lysosomal calcium channel, TRPML1. Thus, my second goal was to determine the role of TRPML1 in macrophages phagocytosis and phagosome maturation. 


\subsubsection{Hypothesis II:}

The phosphoinositide-gated lysosomal $\mathrm{Ca}^{2+}$ channel, TRPML1, is required for phagosome maturation

\section{Objectives:}

1. Test the impact of siRNA silencing of TRPML1 in phagocytosis

2. Determine the role of TRPML1 in phagosome maturation

3. Test and measure calcium dynamics during phagosome maturation

Our results indicated that the level of cytosolic calcium increases during phagosome maturation and this increase is due to the release of lysosomal calcium through TRPML1. In addition, knockdown of TRPML1 blocked increase in cytosolic calcium postphagocytosis interfered with phagosome maturation in macrophages. Specifically, phagosomes and lysosomes were arrested in a docked intermediate that failed to fuse.

To draw a solid conclusion about the role of PIKfyve in immunity, we then tested the role of PIKfyve in other phagocytes such as neutrophils. Therefore, my third goal was to determine the role of PIKfyve in neutrophils immune response.

\subsubsection{Hypothesis III:}

PIKfyve plays an important role in neutrophils immune function 


\section{Objectives:}

1. Determine the role of PIKfyve in neutrophils phagocytosis and phagosome maturation

2. Determine the role of PIKfyve in neutrophils ROS production and chemotaxis

3. Determine a mechanism that explains involvement of PIKfyve in neutrophil immune function

Our data indicates that PIKfyve controls phagosome maturation, chemotaxis and ROS production in neutrophils. Importantly, we show that PIKfyve activity is necessary for Rac GTPase activation. We speculate that $\mathrm{PI}(3,5) \mathrm{P}_{2}$ modulates phagosome maturation, while PI5P, perhaps through recruitment of Rac GEFs such as Tiam1 may stimulate Rac, chemotaxis and ROS production in neutrophils. 


\subsection{References}

Abo, A., Pick, E., Hall, A., Totty, N., Teahan, C. G., \& Segal, A. W. (1991). Activation of the NADPH oxidase involves the small GTP-binding protein p21rac1. Nature, 353(6345), 668-70. http://doi.org/10.1038/353668a0

Ackerman, A. L., \& Cresswell, P. (2003). Regulation of MHC class I transport in human dendritic cells and the dendritic-like cell line KG-1. Journal of Immunology (Baltimore, Md. : 1950), 170(8), 4178-88. http://doi.org/10.4049/jimmunol.170.8.4178

Aderem, A. (2003). Phagocytosis and the inflammatory response. J.Infect.Dis., 187 Supp/(0022-1899 (Print)), S340-S345. http://doi.org/JID21046 $[p i i] \backslash r 10.1086 / 374747$

Akerboom, J., Rivera, J. D. V., Rodríguez Guilbe, M. M., Malavé, E. C. A., Hernandez, H. H., Tian, L., Schreiter, E. R. (2009). Crystal structures of the GCaMP calcium sensor reveal the mechanism of fluorescence signal change and aid rational design. Journal of Biological Chemistry, 284(10), 6455-6464. http://doi.org/10.1074/jbc.M807657200

Akira, S., Uematsu, S., \& Takeuchi, O. (2006). Pathogen Recognition and Innate Immunity. Cell, 124(4), 783-801. http://doi.org/10.1016/j.cell.2006.02.015

Alberts, B., Johnson, A., Lewis, J., Raff, M., Roberts, K., And Walter, P., \& Alberts A.; Lewis, J. et al, B. . J. (2002). Molecular Biology of the cell. 4th edition. Amino Acids (Vol. 54). 
Anderson, K. E., Chessa, T. A. M., Davidson, K., Henderson, R. B., Walker, S., Tolmachova, T., ... Hawkins, P. T. (2010). Ptdlns3P and Rac direct the assembly of the NADPH oxidase on a novel, pre-phagosomal compartment during FcR-mediated phagocytosis in primary mouse neutrophils. Blood, 116(23), 4978-4989. http://doi.org/10.1182/blood-2010-03-275602

Andrews, N. W. (2005). Membrane repair and immunological danger. EMBO Reports, 6(9), 826-830. http://doi.org/10.1038/sj.embor.7400505

Attout, T., Floto, A., \& Launay, P. (2014). Calcium channels in Fc receptor signaling. Current Topics in Microbiology and Immunology, 382, 95-110. http://doi.org/10.1007/978-3-319-07911-0_5

Bajno, L., Peng, X. R., Schreiber, A. D., Moore, H. P., Trimble, W. S., \& Grinstein, S. (2000). Focal exocytosis of VAMP3-containing vesicles at sites of phagosome formation. The Journal of Cell Biology, 149(3), 697-706.

Baker, M. J., Pan, D., \& Welch, H. C. E. (2016). Small GTPases and their guaninenucleotide exchange factors and GTPase-activating proteins in neutrophil recruitment. Current Opinion in Hematology, 23(1), 44-54. http://doi.org/10.1097/MOH.0000000000000199

Balla, T. (2013). Phosphoinositides: tiny lipids with giant impact on cell regulation. Physiological Reviews, 93(3), 1019-137. http://doi.org/10.1152/physrev.00028.2012

Barker, R. (1968). Chapter 3. Ecological Psychology. http://doi.org/10.1016/B978-185617-370-4.50005-1 
Beertsen, W., Willenborg, M., Everts, V., Zirogianni, A., Podschun, R., Schröder, B., Saftig, P. (2008). Impaired phagosomal maturation in neutrophils leads to periodontitis in Iysosomal-associated membrane protein-2 knockout mice. Journal of Immunology (Baltimore, Md. : 1950), 180(1), 475-82.

Benard, V., Bohl, B. P., \& Bokoch, G. M. (1999). Characterization of Rac and Cdc42 activation in chemoattractant- stimulated human neutrophils using a novel assay for active GTPases. Journal of Biological Chemistry, 274(19), 13198-13204. http://doi.org/10.1074/jbc.274.19.13198

Birkeland, H. C. G., \& Stenmark, H. (2004). Protein targeting to endosomes and phagosomes via FYVE and PX domains. Current Topics in Microbiology and Immunology, 282, 89-115.

Boespflug, N. D., Kumar, S., McAlees, J. W., Phelan, J. D., Grimes, H. L., Hoebe, K., Karp, C. L. (2014). ATF3 is a novel regulator of mouse neutrophil migration. Blood, 123(13), 2084-93. http://doi.org/10.1182/blood-2013-06-510909

Borghesi, L., \& Milcarek, C. (2007). Innate versus adaptive immunity: A paradigm past its prime? Cancer Research, 67(9), 3989-3993. http://doi.org/10.1158/00085472.CAN-07-0182

Braun, V., Fraisier, V., Raposo, G., Hurbain, I., Sibarita, J.-B., Chavrier, P., Niedergang, F. (2004). TI-VAMP/VAMP7 is required for optimal phagocytosis of opsonised particles in macrophages. The EMBO Journal, 23(21), 4166-76. http://doi.org/10.1038/sj.emboj.7600427

Bridges, D., Ma, J.-T., Park, S., Inoki, K., Weisman, L. S., \& Saltiel, A. R. (2012). 
Phosphatidylinositol 3,5-bisphosphate plays a role in the activation and subcellular localization of mechanistic target of rapamycin 1. Molecular Biology of the Cell, 23(15), 2955-62. http://doi.org/10.1091/mbc.E11-12-1034

Burgoyne, R. D., \& Clague, M. J. (2003). Calcium and calmodulin in membrane fusion. Biochimica et Biophysica Acta, 1641(2-3), 137-43.

Cai, X., Xu, Y., Cheung, A. K., Tomlinson, R. C., Alcázar-Román, A., Murphy, L., Huang, Q. (2013). PIKfyve, a class III PI Kinase, is the target of the small molecular IL-12/IL-23 inhibitor apilimod and a player in toll-like receptor signaling. Chemistry and Biology, 20(7), 912-921. http://doi.org/10.1016/j.chembiol.2013.05.010

Cai, X., Xu, Y., Kim, Y.-M., Loureiro, J., \& Huang, Q. (2014). PIKfyve, a class III lipid kinase, is required for TLR-induced type I IFN production via modulation of ATF3. Journal of Immunology (Baltimore, Md. : 1950), 192(7), 3383-9.

http://doi.org/10.4049/jimmunol.1302411

Carlier, M. F., Ducruix, A., \& Pantaloni, D. (1999). Signalling to actin: The Cdc42-N-WASPArp2/3 connection. Chemistry and Biology. http://doi.org/10.1016/S10745521(99)80107-0

Carstanjen, D., Yamauchi, A., Koornneef, A., Zang, H., Filippi, M.-D., Harris, C., Williams, D. a. (2005). Rac2 regulates neutrophil chemotaxis, superoxide production, and myeloid colony formation through multiple distinct effector pathways. Journal of Immunology (Baltimore, Md. : 1950), 174(8), 4613-20. http://doi.org/10.4049/jimmunol.174.8.4613

Chakrabarti, S., Andrade, L. O., \& Andrews, N. W. (2005). Trypanosoma cruzi invades 
synaptotagmin VII-deficient cells by a PI-3 kinase independent pathway. Molecular and Biochemical Parasitology, 141(1), 125-128.

http://doi.org/10.1016/j.molbiopara.2005.01.016

Cheng, X., Zhang, X., Gao, Q., Ali Samie, M., Azar, M., Tsang, W. L., Xu, H. (2014). The intracellular $\mathrm{Ca}(2+)$ channel MCOLN1 is required for sarcolemma repair to prevent muscular dystrophy. Nature Medicine, 20(10), 1187-92.

http://doi.org/10.1038/nm.3611

Cieutat, A. M., Lobel, P., August, J. T., Kjeldsen, L., Sengeløv, H., Borregaard, N., \& Bainton, D. F. (1998). Azurophilic granules of human neutrophilic leukocytes are deficient in lysosome-associated membrane proteins but retain the mannose 6phosphate recognition marker. Blood, 91(3), 1044-58.

Clark, A. J., \& Petty, H. R. (2010). A cell permeant peptide containing the cytoplasmic tail sequence of Fc receptor type IIA reduces calcium signaling and phagolysosome formation in neutrophils. Cellular Immunology, 261(2), 153-158.

http://doi.org/10.1016/j.cellimm.2009.12.002

Colletti, G. A., Miedel, M. T., Andharia, N., Weisz, O. A., \& Kiselyov, K. (2012). Loss of Lysosomal Ion Channel Transient Receptor Potential Channel Mucolipin-1 ( TRPML1 ) Leads to Cathepsin, 1. http://doi.org/10.1074/jbc.M111.285536

Coutinho, M. F., Prata, M. J., \& Alves, S. (2012). Mannose-6-phosphate pathway: A review on its role in lysosomal function and dysfunction. Molecular Genetics and Metabolism. http://doi.org/10.1016/j.ymgme.2011.12.012

Cox, D., Berg, J. S., Cammer, M., Chinegwundoh, J. O., Dale, B. M., Cheney, R. E., \& 
Greenberg, S. (2002). Myosin X is a downstream effector of $\mathrm{PI}(3) \mathrm{K}$ during phagocytosis. Nature Cell Biology, 4(7), 469-477. http://doi.org/10.1038/ncb805 Cuervo, A. M., \& Dice, J. F. (2000). Regulation of Lamp2a Levels in the Lysosomal Membrane. Traffic, 1, 570-583. http://doi.org/tra010707 [pii]

Cunningham, C. C., Vegners, R., Bucki, R., Funaki, M., Korde, N., Hartwig, J. H., Janmey, P. A. (2001). Cell permeant polyphosphoinositide-binding peptides that block cell motility and actin assembly. The Journal of Biological Chemistry, 276(46), 43390-9. http://doi.org/10.1074/jbc.M105289200

Czibener, C., Sherer, N. M., Becker, S. M., Pypaert, M., Hui, E., Chapman, E. R., Andrews, N. W. (2006). Ca2+ and synaptotagmin VII-dependent delivery of lysosomal membrane to nascent phagosomes. The Journal of Cell Biology, 174(7), 997-1007. http://doi.org/10.1083/jcb.200605004

Davies, L. C., Jenkins, S. J., Allen, J. E., \& Taylor, P. R. (2013). Tissue-resident macrophages. Nature Immunology, 14(10), 986-95. http://doi.org/10.1038/ni.2705 Dayam, R. M., Saric, A., Shilliday, R. E., \& Botelho, R. J. (2015). The PhosphoinositideGated Lysosomal Ca(2+) Channel, TRPML1, Is Required for Phagosome Maturation. Traffic (Copenhagen, Denmark), 16(9), 1010-26. http://doi.org/10.1111/tra.12303 de Back, D. Z., Kostova, E. B., van Kraaij, M., van den Berg, T. K., \& van Bruggen, R. (2014). Of macrophages and red blood cells; A complex love story. Frontiers in Physiology. http://doi.org/10.3389/fphys.2014.00009

de Lartigue, J., Polson, H., Feldman, M., Shokat, K., Tooze, S. A., Urbé, S., \& Clague, M. J. (2009). PIKfyve regulation of endosome-linked pathways. Traffic (Copenhagen, 
Denmark), 10(7), 883-93.

Dell'Angelica, E. C., Mullins, C., Caplan, S., \& Bonifacino, J. S. (2000). Lysosome-related organelles. FASEB Journal : Official Publication of the Federation of American Societies for Experimental Biology, 14(10), 1265-78.

Di Paolo, G., \& De Camilli, P. (2006). Phosphoinositides in cell regulation and membrane dynamics. Nature, 443(7112), 651-657. http://doi.org/10.1038/nature05185

Dong, X., Shen, D., Wang, X., Dawson, T., Li, X., Zhang, Q., ... Chen, X. (2010). PI(3,5)P 2 Mucolipin Ca2+ Controls Membrane Traffic by Direct Activation of Release Channels in the Endolysosome. Nature Communications, 1(4), 1-21. http://doi.org/10.1038/ncomms1037.PI)

Dong, X., Shen, D., Wang, X., Dawson, T., Li, X., Zhang, Q., Xu, H. (2010). PI(3,5)P(2) controls membrane trafficking by direct activation of mucolipin $\mathrm{Ca}(2+)$ release channels in the endolysosome. Nature Communications, 1, 38. http://doi.org/10.1038/ncomms1037

Dong, X., Wang, X., Shen, D., Chen, S., Liu, M., Wang, Y., Xu, H. (2009). Activating mutations of the TRPML1 channel revealed by proline-scanning mutagenesis. The Journal of Biological Chemistry, 284(46), 32040-52.

http://doi.org/10.1074/jbc.M109.037184

Efeyan, A., Zoncu, R., \& Sabatini, D. M. (2012). Amino acids and mTORC1: From lysosomes to disease. Trends in Molecular Medicine. http://doi.org/10.1016/j.molmed.2012.05.007

El Benna, J., Ruedi, J. M., \& Babior, B. M. (1994). Cytosolic Guanine Nucleotide-binding 
Protein Rac2 Operates in Vivo as a Component of the Neutrophil Respiratory Burst Oxidase: Transfer of Rac2 and the cytosolic oxidase components p47phox and p67phox to the submembranous actin cytoskeleton during oxidase. Journal of Biological Chemistry, 269(9), 6729-6734.

Elhelu, M. A. (1983). The role of macrophages in immunology. Journal of the National Medical Association, 75(3), 314-7. Retrieved from http://www.pubmedcentral.nih.gov/articlerender.fcgi?artid=2561478\&tool pmcentrez\&rendertype=abstract

Ellson, C. D., Anderson, K. E., Morgan, G., Chilvers, E. R., Lipp, P., Stephens, L. R., \& Hawkins, P. T. (2001). Phosphatidylinositol 3-phosphate is generated in phagosomal membranes. Current Biology : CB, 11(20), 1631-5.

Ellson, C. D., Gobert-Gosse, S., Anderson, K. E., Davidson, K., Erdjument-Bromage, H., Tempst, P., Stephens, L. R. (2001). Ptdlns(3)P regulates the neutrophil oxidase complex by binding to the PX domain of p40(phox). Nature Cell Biology, 3(7), 67982. http://doi.org/10.1038/35083076

Ellson, C., Davidson, K., Anderson, K., Stephens, L. R., \& Hawkins, P. T. (2006). Ptdlns3P binding to the PX domain of p40phox is a physiological signal in NADPH oxidase activation. The EMBO Journal, 25(19), 4468-78. http://doi.org/10.1038/sj.emboj.7601346

Eskelinen, E. L., Tanaka, Y., \& Saftig, P. (2003). At the acidic edge: Emerging functions for Iysosomal membrane proteins. Trends in Cell Biology. http://doi.org/10.1016/S0962-8924(03)00005-9 
Fairn, G. D., \& Grinstein, S. (2012). How nascent phagosomes mature to become phagolysosomes. Trends in Immunology, 33(8), 397-405. http://doi.org/10.1016/j.it.2012.03.003

Falkenburger, B. H., Jensen, J. B., Dickson, E. J., Suh, B. C., \& Hille, B. (2010). Phosphoinositides: lipid regulators of membrane proteins. J Physiol, 588(Pt 17), 3179-3185. http://doi.org/10.1113/jphysiol.2010.192153

Faurschou, M., \& Borregaard, N. (2003). Neutrophil granules and secretory vesicles in inflammation. Microbes and Infection, 5(14), 1317-1327. http://doi.org/10.1016/j.micinf.2003.09.008

Faurschou, M., Sørensen, O. E., Johnsen, A. H., Askaa, J., \& Borregaard, N. (2002). Defensin-rich granules of human neutrophils: Characterization of secretory properties. Biochimica et Biophysica Acta - Molecular Cell Research, 1591(1-3), 2935. http://doi.org/10.1016/S0167-4889(02)00243-4

Ferguson, C. J., Lenk, G. M., Jones, J. M., Grant, A. E., Winters, J. J., Dowling, J. J., Meisler, M. H. (2012). Neuronal expression of Fig4 is both necessary and sufficient to prevent spongiform neurodegeneration. Human Molecular Genetics, 21(16), 352534. http://doi.org/10.1093/hmg/dds179

Flannagan, R. S., Cosío, G., \& Grinstein, S. (2009). Antimicrobial mechanisms of phagocytes and bacterial evasion strategies. Nature Reviews. Microbiology, 7(5), 355-66. http://doi.org/10.1038/nrmicro2128

Flannagan, R. S., Jaumouillé, V., \& Grinstein, S. (2012). The cell biology of phagocytosis. Annual Review of Pathology, 7, 61-98. http://doi.org/10.1146/annurev-pathol- 


\section{1-132445}

Fratti, R. A., Backer, J. M., Gruenberg, J., Corvera, S., \& Deretic, V. (2001). Role of phosphatidylinositol 3-kinase and Rab5 effectors in phagosomal biogenesis and mycobacterial phagosome maturation arrest. The Journal of Cell Biology, 154(3), 631-44. http://doi.org/10.1083/jcb.200106049

Garcl, E., Rosales C. (2002). Signal transduction during Fc receptor-mediated phagocytosis. journal of Leukocyte Biology. 72 (6), 1092-1108.

Gary, J. D., Wurmser, A. E., Bonangelino, C. J., Weisman, L. S., \& Emr, S. D. (1998). Fab1p is essential for Ptdlns(3)P 5-kinase activity and the maintenance of vacuolar size and membrane homeostasis. Journal of Cell Biology, 143(1), 65-79. http://doi.org/10.1083/jcb.143.1.65

Gerasimenko, J. V, Gerasimenko, O. V, \& Petersen, O. H. (2001). Membrane repair: $\mathrm{Ca}(2+)$-elicited lysosomal exocytosis. Current Biology : $C B, 11(23), \mathrm{R} 971-4$. Gillooly, D. J., Morrow, I. C., Lindsay, M., Gould, R., Bryant, N. J., Gaullier, J. M., Stenmark, H. (2000). Localization of phosphatidylinositol 3-phosphate in yeast and mammalian cells. The EMBO Journal, 19(17), 4577-88. http://doi.org/10.1093/emboj/19.17.4577

Glogauer, M., Marchal, C. C., Zhu, F., Worku, A., Clausen, Foerster, I., Kwiatkowski, D. J. (2003). Rac1 deletion in mouse neutrophils has selective effects on neutrophil functions. J Immunol, 170(11), 5652-7. http://doi.org/10.4049/jimmunol.170.11.5652

Gold, E. S., Morrissette, N. S., Underhill, D. M., Guo, J., Bassetti, M., \& Aderem, a. 
(2000). Amphiphysin IIm, a novel amphiphysin II isoform, is required for

macrophage phagocytosis. Immunity, 12(3), 285-292.

http://doi.org/10.1016/S1074-7613(00)80181-8

Gordon-Smith, T. (2007). Red blood cells. Surgery (Oxford), 25(2), 57-60.

http://doi.org/10.1016/j.mpsur.2006.12.004

Gordon, S. (2003). Alternative activation of macrophages. Nat.Rev.Immunol., 3(14741733 (Print)), 23-35. http://doi.org/10.1038/nri978

Grosshans, B. L., Ortiz, D., \& Novick, P. (2006). Rabs and their effectors: achieving specificity in membrane traffic. Proceedings of the National Academy of Sciences of the United States of America, 103(32), 11821-7. http://doi.org/10.1073/pnas.0601617103

Hannigan, M., Zhan, L., Li, Z., Ai, Y., Wu, D., \& Huang, C.-K. (2002). Neutrophils lacking phosphoinositide 3-kinase gamma show loss of directionality during $\mathrm{N}$-formyl-MetLeu-Phe-induced chemotaxis. Proceedings of the National Academy of Sciences of the United States of America, 99(6), 3603-8.

http://doi.org/10.1073/pnas.052010699

Harrison, R. E., Bucci, C., Vieira, O. V, Schroer, T. A., \& Grinstein, S. (2003). Phagosomes fuse with late endosomes and/or lysosomes by extension of membrane protrusions along microtubules: role of Rab7 and RILP. Molecular and Cellular Biology, 23(18), 6494-506. http://doi.org/10.1128/MCB.23.18.6494-6506.2003

Hay, J. C. (2007). Calcium: a fundamental regulator of intracellular membrane fusion?, 8(3). http://doi.org/10.1038/sj.embor.7400921 
Hazeki, K., Nigorikawa, K., Takaba, Y., Segawa, T., Nukuda, A., Masuda, A., ... Hazeki, O. (2012). Essential roles of PIKfyve and PTEN on phagosomal phosphatidylinositol 3phosphate dynamics. FEBS Letters, 586(22), 4010-5. http://doi.org/10.1016/j.febslet.2012.09.043

Heyworth, P. G., Knaus, U. G., Settleman, J., Curnutte, J. T., \& Bokoch, G. M. (1993).

Regulation of NADPH oxidase activity by Rac GTPase activating protein(s). Molecular Biology of the Cell, 4(11), 1217-1223. Retrieved from http://www.ncbi.nlm.nih.gov/pmc/articles/PMC275755/pdf/mbc00056-0140.pdf Ho, C. Y., Alghamdi, T. A., \& Botelho, R. J. (2012). Phosphatidylinositol-3,5-bisphosphate: no longer the poor PIP2. Traffic (Copenhagen, Denmark), 13(1), 1-8. http://doi.org/10.1111/j.1600-0854.2011.01246.x

Ho, C. Y., Choy, C. H., \& Botelho, R. J. (2016). Radiolabeling and Quantification of Cellular Levels of Phosphoinositides by High Performance Liquid Chromatography-coupled Flow Scintillation. Journal of Visualized Experiments : JoVE, (107).

http://doi.org/10.3791/53529

Huotari, J., \& Helenius, A. (2011). Endosome maturation. The EMBO Journal, 30(17), 3481-3500. http://doi.org/10.1038/emboj.2011.286

Huynh, K. K., Eskelinen, E., Scott, C. C., Malevanets, A., Saftig, P., \& Grinstein, S. (2007). LAMP proteins are required for fusion of lysosomes with phagosomes, 26(2), 313324. http://doi.org/10.1038/sj.emboj.7601511

Ikonomov, O. C., Sbrissa, D., Delvecchio, K., Xie, Y., Jin, J. P., Rappolee, D., \& Shisheva, A. (2011). The phosphoinositide kinase PIKfyve is vital in early embryonic 
development: Preimplantation lethality of PIKfyve-/- embryos but normality of PIKfyve+/- mice. Journal of Biological Chemistry, 286(15), 13404-13413. http://doi.org/10.1074/jbc.M111.222364

Ikonomov, O. C., Sbrissa, D., Fenner, H., \& Shisheva, A. (2009). PIKfyve-ArPIKfyve-Sac3 core complex: Contact sites and their consequence for Sac3 phosphatase activity and endocytic membrane homeostasis. Journal of Biological Chemistry, 284(51), 35794-35806. http://doi.org/10.1074/jbc.M109.037515

Ikonomov, O. C., Sbrissa, D., Foti, M., Carpentier, J.-L., \& Shisheva, A. (2003). PIKfyve controls fluid phase endocytosis but not recycling/degradation of endocytosed receptors or sorting of procathepsin D by regulating multivesicular body morphogenesis. Molecular Biology of the Cell, 14(11), 4581-91.

http://doi.org/10.1091/mbc.E03-04-0222

Ikonomov, O. C., Sbrissa, D., Mlak, K., Kanzaki, M., Pessin, J., \& Shisheva, A. (2002). Functional dissection of lipid and protein kinase signals of PIKfyve reveals the role of Ptdlns 3,5-P2 production for endomembrane integrity. The Journal of Biological Chemistry, 277(11), 9206-11. http://doi.org/10.1074/jbc.M108750200

Ikonomov, O. C., Sbrissa, D., \& Shisheva, A. (2001). Mammalian cell morphology and endocytic membrane homeostasis require enzymatically active phosphoinositide 5kinase PIKfyve. The Journal of Biological Chemistry, 276(28), 26141-7. http://doi.org/10.1074/jbc.M101722200 Italiani, P., \& Boraschi, D. (2014). From monocytes to M1/M2 macrophages: Phenotypical vs. functional differentiation. Frontiers in Immunology, 5(OCT), 1-22. 
http://doi.org/10.3389/fimmu.2014.00514

Jaconi, M. E., Lew, D. P., Carpentier, J. L., Magnusson, K. E., Sjögren, M., \& Stendahl, O. (1990). Cytosolic free calcium elevation mediates the phagosome-lysosome fusion during phagocytosis in human neutrophils. The Journal of Cell Biology, 110(5), $1555-64$.

Jankowski, A., Scott, C. C., \& Grinstein, S. (2002). Determinants of the phagosomal pH in neutrophils. Journal of Biological Chemistry, 277(8), 6059-6066. http://doi.org/10.1074/jbc.M110059200

Jayachandran, R., Sundaramurthy, V., Combaluzier, B., Mueller, P., Korf, H., Huygen, K., ... Pieters, J. (2007). Survival of mycobacteria in macrophages is mediated by coronin 1-dependent activation of calcineurin. Cell, 130(1), 37-50. http://doi.org/10.1016/j.cell.2007.04.043 Jefferies, H. B. J., Cooke, F. T., Jat, P., Boucheron, C., Koizumi, T., Hayakawa, M., ... Parker, P. J. (2008). A selective PIKfyve inhibitor blocks Ptdlns(3,5)P(2) production and disrupts endomembrane transport and retroviral budding. EMBO Reports, 9(2), 164-170. http://doi.org/10.1038/sj.embor.7401155

Karacsonyi, C., San Miguel, A., \& Puertollano, R. (2007). Mucolipin-2 localizes to the Arf6-associated pathway and regulates recycling of GPI-APs. Traffic, 8(10), 14041414. http://doi.org/10.1111/j.1600-0854.2007.00619.x

Kawano, Y., Kaneko-Kawano, T., Shimamoto, K., Hückelhoven, R., \& Zhang, Y. (2014). Rho family GTPase-dependent immunity in plants and animals. Frontiers in Plant Science, 5, 522. http://doi.org/10.3389/fpls.2014.00522 
Kiefer, F., Brumell, J., Al-Alawi, N., Latour, S., Cheng, A., Veillette, A., Pawson, T. (1998). The Syk protein tyrosine kinase is essential for Fcgamma receptor signaling in macrophages and neutrophils. Molecular and Cellular Biology, 18(7), 4209-20. Retrieved from:

http://www.pubmedcentral.nih.gov/articlerender.fcgi?artid=109005\&tool=pmcent rez\&rendertype=abstract

Kielian, M. C., \& Cohn, Z. A. (1980). Phagosome-lysosome fusion. Characterization of intracellular membrane fusion in mouse macrophages. The Journal of Cell Biology, 85(3), 754-65.

Kim, G. H. E., Dayam, R. M., Prashar, A., Terebiznik, M., \& Botelho, R. J. (2014). PIKfyve Inhibition Interferes with Phagosome and Endosome Maturation in Macrophages, (3), 1-21. http://doi.org/10.1111/tra.12199

Kim, H. J., Soyombo, A. A., Tjon-Kon-Sang, S., So, I., \& Muallem, S. (2009). The Ca2+ channel TRPML3 regulates membrane trafficking and autophagy. Traffic, 10(8), 1157-1167. http://doi.org/10.1111/j.1600-0854.2009.00924.x

Kinchen, J. M., \& Ravichandran, K. S. (2008). Phagosome maturation: going through the acid test. Nature Reviews. Molecular Cell Biology, 9(10), 781-95.

http://doi.org/10.1038/nrm2515

Kiselyov, K. K., Ahuja, M., Rybalchenko, V., Patel, S., \& Muallem, S. The intracellular $\mathrm{Ca}^{2+}$ channels of membrane traffic. Channels (Austin, Tex.), 6(5), 344-51. http://doi.org/10.4161/chan.21723

Klebanoff, S. J. (1999). Myeloperoxidase. Proc Assoc Am Physicians, 111(5), 383-389. 
Knaus, U. G., Heyworth, P. G., Evans, T., Curnutte, J. T., \& Bokoch, G. M. (1991).

Regulation of phagocyte oxygen radical production by the GTP-binding protein Rac 2. Science (New York, N.Y.), 254(5037), 1512-5.

Koh, A. L. Y., Sun, C. X., Zhu, F., \& Glogauer, M. (2005). The role of Rac1 and Rac2 in bacterial killing. Cellular Immunology, 235(2), 92-97. http://doi.org/10.1016/j.cellimm.2005.07.005

Kornfeld, S., \& Mellman, I. (1989). The biogenesis of lysosomes. Annual Review of Cell Biology, 5, 483-525. http://doi.org/10.1146/annurev.cb.05.110189.002411

Krajcovic, M., Krishna, S., Akkari, L., Joyce, J. a, \& Overholtzer, M. (2013). mTOR regulates phagosome and entotic vacuole fission. Molecular Biology of the Cell, 24(23), 3736-3745. http://doi.org/10.1091/mbc.E13-07-0408

Lee, W. L., Harrison, R. E., \& Grinstein, S. (2003a). Phagocytosis by neutrophils. Microbes and Infection. http://doi.org/10.1016/j.micinf.2003.09.014

Lee, W. L., Harrison, R. E., \& Grinstein, S. (2003b). Phagocytosis by neutrophils. Microbes and Infection, 5(14), 1299-1306.

Lemmon, M. A. (2008). Membrane recognition by phospholipid-binding domains. Nature Reviews. Molecular Cell Biology, 9(2), 99-111. http://doi.org/10.1038/nrm2328

Lew, P. D., Monod, A., Waldvogel, F. A., Dewald, B., Baggiolini, M., \& Pozzan, T. (1986). Quantitative analysis of the cytosolic free calcium dependency of exocytosis from three subcellular compartments in intact human neutrophils. The Journal of Cell Biology, 102(6), 2197-204. 
Li, X., Wang, X., Zhang, X., Zhao, M., Tsang, W. L., Zhang, Y., Xu, H. (2013). Genetically encoded fluorescent probe to visualize intracellular phosphatidylinositol 3,5bisphosphate localization and dynamics. Proceedings of the National Academy of Sciences of the United States of America, 110(52), 21165-70. http://doi.org/10.1073/pnas.1311864110

Lietha, D. (2011). Phosphoinositides - The Seven Species: Conversion and Cellular Roles. In Encyclopedia of Life Sciences (pp. 1-11). http://doi.org/10.1002/9780470015902.a0023177

Madsen, S. J., Baek, S. K., Makkouk, A. R., Krasieva, T., \& Hirschberg, H. (2012). Macrophages as cell-based delivery systems for nanoshells in photothermal therapy. Annals of Biomedical Engineering, 40(2), 507-515. http://doi.org/10.1007/s10439-011-0415-1

Makita, N., Hizukuri, Y., Yamashiro, K., \& Murakawa, M. (2014). IL-10 enhances the phenotype of $\mathrm{M} 2$ macrophages induced by IL-4 and confers the ability to increase eosinophil migration, 27(3), 131-141. http://doi.org/10.1093/intimm/dxu090 Malik, Z. A., Denning, G. M., \& Kusner, D. J. (2000). Inhibition of $\mathrm{Ca}(2+)$ signaling by Mycobacterium tuberculosis is associated with reduced phagosome-lysosome fusion and increased survival within human macrophages. The Journal of Experimental Medicine, 191(2), 287-302.

Malik, Z. A., Thompson, C. R., Hashimi, S., Porter, B., lyer, S. S., \& Kusner, D. J. (2003). Cutting edge: Mycobacterium tuberculosis blocks Ca2+ signaling and phagosome maturation in human macrophages via specific inhibition of sphingosine kinase. 
Journal of Immunology (Baltimore, Md. : 1950), 170(6), 2811-5.

Martin, S., Harper, C. B., May, L. M., Coulson, E. J., Meunier, F. A., \& Osborne, S. L. (2013). Inhibition of PIKfyve by YM-201636 dysregulates autophagy and leads to apoptosis-independent neuronal cell death. PloS One, 8(3), e60152.

http://doi.org/10.1371/journal.pone.0060152

Mayadas, T. N., Cullere, X., \& Lowell, C. A. (2014). The multifaceted functions of neutrophils. Annual Review of Pathology, 9, 181-218.

McCartney, A. J., Zhang, Y., \& Weisman, L. S. (2014). Phosphatidylinositol 3,5-

bisphosphate: low abundance, high significance. BioEssays : News and Reviews in Molecular, Cellular and Developmental Biology, 36(1), 52-64.

http://doi.org/10.1002/bies.201300012

Mellman, I. (1996). Endocytosis and molecular sorting. Annual Review of Cell and Developmental Biology, 12, 575-625. http://doi.org/10.1146/annurev.cellbio.12.1.575

Min, S. H., Suzuki, A., Stalker, T. J., Zhao, L., Wang, Y., McKennan, C., Abrams, C. S. (2014a). Loss of PIKfyve in platelets causes a lysosomal disease leading to inflammation and thrombosis in mice. Nature Communications, 5(May), 4691. http://doi.org/10.1038/ncomms5691

Min, S. H., Suzuki, A., Stalker, T. J., Zhao, L., Wang, Y., McKennan, C., Abrams, C. S. (2014b). Loss of PIKfyve in platelets causes a lysosomal disease leading to inflammation and thrombosis in mice. Nature Communications, 5, 4691. http://doi.org/10.1038/ncomms5691 
Minakami, R., Maehara, Y., Kamakura, S., Kumano, O., Miyano, K., \& Sumimoto, H. (2010). Membrane phospholipid metabolism during phagocytosis in human neutrophils. Genes to Cells : Devoted to Molecular \& Cellular Mechanisms, 15(5), 409-24.

Mitchell, M. A., Huang, M. M., Chien, P., Indik, Z. K., Pan, X. Q., \& Schreiber, A. D. (1994). Substitutions and deletions in the cytoplasmic domain of the phagocytic receptor Fc gamma RIIA: effect on receptor tyrosine phosphorylation and phagocytosis. Blood, 84(6), 1753-9. Retrieved from http://www.ncbi.nlm.nih.gov/pubmed/7521687

Mizrahi, A., Molshanski-Mor, S., Weinbaum, C., Zheng, Y., Hirshberg, M., \& Pick, E. (2005). Activation of the phagocyte NADPH oxidase by Rac Guanine nucleotide exchange factors in conjunction with ATP and nucleoside diphosphate kinase. The Journal of Biological Chemistry, 280(5), 3802-11. http://doi.org/10.1074/jbc.M410257200

Mrakovic, A., Kay, J. G., Furuya, W., Brumell, J. H., \& Botelho, R. J. (2012). Rab7 and Arl8 GTPases are Necessary for Lysosome Tubulation in Macrophages. Traffic, 13(12), 1667-1679. http://doi.org/10.1111/tra.12003

Munoz, C., Almilaji, A., Setiawan, I., Föller, M., \& Lang, F. (2013). Up-regulation of the inwardly rectifying $\mathrm{K}^{+}$channel Kir2.1 (KCNJ2) by protein kinase $\mathrm{B}$ (PKB/Akt) and PIKfyve. The Journal of Membrane Biology, 246(3), 189-97. http://doi.org/10.1007/s00232-012-9520-9 Muraille, E., Leo, O., \& Moser, M. (2014). Th1/Th2 paradigm extended: Macrophage 
polarization as an unappreciated pathogen-driven escape mechanism? Frontiers in Immunology. http://doi.org/10.3389/fimmu.2014.00603

Murray, P. J., \& Wynn, T. A. (2011). Protective and pathogenic functions of macrophage subsets. Nature Reviews Immunology, 11(11), 723-737. http://doi.org/Doi 10.1038/Nri3073

Nauseef, W. M., \& Borregaard, N. (2014). Neutrophils at work. Nature Immunology, 15(7), 602-11. http://doi.org/10.1038/ni.2921

Newton, K., \& Dixit, V. M. (2012). Signaling in Innate Immunity and Inflammation. Cold Spring Harb Perspect Biol. 4, 006049.

Nicot, A.-S., Fares, H., Payrastre, B., Chisholm, A. D., Labouesse, M., \& Laporte, J. (2006). The phosphoinositide kinase PIKfyve/Fab1p regulates terminal lysosome maturation in Caenorhabditis elegans. Molecular Biology of the Cell, 17, 30623074. http://doi.org/10.1091/mbc.E05-12-1120

Niessen, H. W., Kuijpers, T. W., Roos, D., \& Verhoeven, A. J. (1991). Release of azurophilic granule contents in fMLP-stimulated neutrophils requires two activation signals, one of which is a rise in cytosolic free Ca2+. Cellular Signalling, $3(6), 625-33$.

Nordenfelt, P., \& Tapper, H. (2011). Phagosome dynamics during phagocytosis by neutrophils. Journal of Leukocyte Biology, 90(August), 271-284. http://doi.org/10.1189/jlb.0810457 Nordmann, M., Cabrera, M., Perz, A., Br??cker, C., Ostrowicz, C., Engelbrecht-Vandr??, S., \& Ungermann, C. (2010). The Mon1-Ccz1 complex is the GEF of the late 
endosomal Rab7 homolog Ypt7. Current Biology, 20(18), 1654-1659.

http://doi.org/10.1016/j.cub.2010.08.002

Oppelt, A., Haugsten, E. M., Zech, T., Danielsen, H. E., Sveen, A., Lobert, V. H., ... Wesche, J. (2014). PIKfyve, MTMR3 and their product PtdIns5P regulate cancer cell migration and invasion through activation of Rac1. The Biochemical Journal, 461(3), 383-90. http://doi.org/10.1042/BJ20140132

Oppelt, A., Lobert, V. H., Haglund, K., Mackey, A. M., Rameh, L. E., Liestøl, K., ... Wesche, J. (2013). Production of phosphatidylinositol 5-phosphate via PIKfyve and MTMR3 regulates cell migration. EMBO Reports, 14(1), 57-64.

http://doi.org/10.1038/embor.2012.183

Osborne, S. L., Wen, P. J., Boucheron, C., Nguyen, H. N., Hayakawa, M., Kaizawa, H., ... Meunier, F. a. (2008). PIKfyve negatively regulates exocytosis in neurosecretory cells. Journal of Biological Chemistry, 283(5), 2804-2813. http://doi.org/10.1074/jbc.M704856200

Pakladok, T., Almilaji, A., Munoz, C., Alesutan, I., \& Lang, F. (2013). PIKfyve sensitivity of hERG channels. Cellular Physiology and Biochemistry : International Journal of Experimental Cellular Physiology, Biochemistry, and Pharmacology, 31(6), 785-94. http://doi.org/10.1159/000350096

Palmer, C., Diehn, M., Alizadeh, A. a, \& Brown, P. O. (2006). Cell-type specific gene expression profiles of leukocytes in human peripheral blood. BMC Genomics, 7, 115. http://doi.org/10.1186/1471-2164-7-115.

Pesanti, E. L., \& Axline, S. G. (1975). Phagolysosome formation in normal and colchicine- 
treated macrophages. The Journal of Experimental Medicine, 142(4), 903-13.

Pryor, P. R., Mullock, B. M., Bright, N. A., Gray, S. R., \& Luzio, J. P. (2000). The role of intraorganellar $\mathrm{Ca}(2+)$ in late endosome-lysosome heterotypic fusion and in the reformation of lysosomes from hybrid organelles. The Journal of Cell Biology, 149(5), 1053-62.

Pryor, P. R., Reimann, F., Gribble, F. M., \& Luzio, J. P. (2006). Mucolipin-1 Is a Lysosomal Membrane Protein Required for Intracellular Lactosylceramide Traffic, 1388-1398. http://doi.org/10.1111/j.1600-0854.2006.00475.x

Reddy, A., Caler, E. V, \& Andrews, N. W. (2001). Plasma membrane repair is mediated by $\mathrm{Ca}(2+)$-regulated exocytosis of lysosomes. Cell, 106(2), 157-69.

Roberts, A. W., Kim, C., Zhen, L., Lowe, J. B., Kapur, R., Petryniak, B., ... Williams, D. A. (1999). Deficiency of the Hematopoietic Cell-Specific Rho Family GTPase Rac2 Is Characterized by Abnormalities in Neutrophil Function and Host Defense. Immunity, 10(2), 183-196. http://doi.org/10.1016/S1074-7613(00)80019-9

Rohatgi, R., Ho, H. Y. H., \& Kirschner, M. W. (2000). Mechanism of N-WASP activation by CDC42 and phosphatidylinositol 4,5-bisphosphate. Journal of Cell Biology, 150(6), 1299-1309. http://doi.org/10.1083/jcb.150.6.1299

Rong, Y., Liu, M., Ma, L., Du, W., Zhang, H., Tian, Y., ... Yu, L. (2012). Clathrin and phosphatidylinositol-4,5-bisphosphate regulate autophagic lysosome reformation. Nature Cell Biology, 14(9), 924-934. http://doi.org/10.1038/ncb2557

Rutherford, A. C., Traer, C., Wassmer, T., Pattni, K., Bujny, M. V, Carlton, J. G., ... Cullen, P. J. (2006). The mammalian phosphatidylinositol 3-phosphate 5-kinase (PIKfyve) 
regulates endosome-to-TGN retrograde transport. Journal of Cell Science, 119(Pt 19), 3944-57. http://doi.org/10.1242/jcs.03153

Saarikangas, J., Zhao, H., \& Lappalainen, P. (2010). Regulation of the actin cytoskeletonplasma membrane interplay by phosphoinositides. Physiological Reviews, 90, 259289. http://doi.org/10.1152/physrev.00036.2009

Sadhu, C., Masinovsky, B., Dick, K., Sowell, C. G., \& Staunton, D. E. (2003). Essential role of phosphoinositide 3-kinase delta in neutrophil directional movement. Journal of Immunology (Baltimore, Md. : 1950), 170(5), 2647-54.

Saftig, P., \& Klumperman, J. (2009). Lysosome biogenesis and lysosomal membrane proteins: trafficking meets function. Nature Reviews. Molecular Cell Biology, 10(9), 623-635. http://doi.org/10.1038/nrm2745

Samie, M. A., \& Xu, H. (n.d.). Lysosomal Exocytosis and Lipid Storage Disorders.

Samie, M., Wang, X., Zhang, X., Goschka, A., Li, X., Cheng, X., ... Ferrer, M. (2013). Article A TRP Channel in the Lysosome Regulates Large Particle Phagocytosis via Focal Exocytosis. Developmental Cell, 1-14. http://doi.org/10.1016/j.devcel.2013.08.003

Sbrissa, D., Ikonomov, O. C., Filios, C., Delvecchio, K., \& Shisheva, A. (2012). Functional dissociation between PIKfyve-synthesized Ptdlns5P and PI(3,5)P2 by means of the PIKfyve inhibitor YM201636. American Journal of Physiology. Cell Physiology, 303(4), C436-46. http://doi.org/10.1152/ajpcell.00105.2012

Sbrissa, D., Ikonomov, O. C., \& Shisheva, A. (2000). PIKfyve lipid kinase is a protein kinase: Downregulation of 5???-phosphoinositide product formation by autophosphorylation. Biochemistry, 39(51), 15980-15989. 
http://doi.org/10.1021/bi001897f

Scott, C. C., Botelho, R. J., \& Grinstein, S. (2003). Phagosome maturation: A few bugs in the system. Journal of Membrane Biology. http://doi.org/10.1007/s00232-0022008-2

Segal, A. W. (2008). The function of the NADPH oxidase of phagocytes and its relationship to other NOXs in plants, invertebrates, and mammals. International Journal of Biochemistry and Cell Biology. http://doi.org/10.1016/j.biocel.2007.10.003

Segal, A. W., Dorling, J., \& Coade, S. (1980). Kinetics of fusion of the cytoplasmic granules with phagocytic vacuoles in human polymorphonuclear leukocytes. Biochemical and morphological studies. Journal of Cell Biology, 85(1), 42-59. http://doi.org/10.1083/jcb.85.1.42

Sengeløv, H., Kjeldsen, L., Diamond, M. S., Springer, T. a, \& Borregaard, N. (1993). Subcellular localization and dynamics of Mac-1 (alpha m beta 2 ) in human neutrophils. The Journal of Clinical Investigation, 92(3), 1467-1476. http://doi.org/10.1172/JCl116724

Shen, D., Wang, X., Li, X., Zhang, X., Yao, Z., Dibble, S., ... Xu, H. (2012). Lipid storage disorders block lysosomal trafficking by inhibiting a TRP channel and lysosomal calcium release. Nature Communications, 3, 731. http://doi.org/10.1038/ncomms1735

Sheshachalam, A., Srivastava, N., Mitchell, T., Lacy, P., \& Eitzen, G. (2014). Granule protein processing and regulated secretion in neutrophils. Frontiers in Immunology, 
5, 448. http://doi.org/10.3389/fimmu.2014.00448

Shisheva, A., Sbrissa, D., \& Ikonomov, O. (2015). Plentiful Ptdlns5P from scanty PI(3,5)P2 or from ample PtdIns? PIKfyve-dependent models: Evidence and speculation (response to: DOI 10.1002/bies.201300012). BioEssays : News and Reviews in Molecular, Cellular and Developmental Biology, 37(3), 267-77.

http://doi.org/10.1002/bies.201400129

Sobota, A., Strzelecka-Kiliszek, A., Gładkowska, E., Yoshida, K., Mrozińska, K., \& Kwiatkowska, K. (2005). Binding of IgG-opsonized particles to Fc gamma R is an active stage of phagocytosis that involves receptor clustering and phosphorylation. Journal of Immunology (Baltimore, Md. : 1950), 175, 4450-4457. http://doi.org/10.4049/jimmunol.175.7.4450

Soyombo, A. A., Tjon-kon-sang, S., Rbaibi, Y., Bashllari, E., Muallem, S., Soyombo, A. A., Bisceglia, J. (2006). TRP-ML1 Regulates Lysosomal pH and Acidic Lysosomal Lipid Hydrolytic Activity *. http://doi.org/10.1074/jbc.M508211200

Steinberg, B. E., \& Grinstein, S. (2008). Pathogen destruction versus intracellular survival: The role of lipids as phagosomal fate determinants. Journal of Clinical Investigation. http://doi.org/10.1172/JCl35433

Su, D. L., Lu, Z. M., Shen, M. N., Li, X., \& Sun, L. Y. (2012). Roles of pro- and antiinflammatory cytokines in the pathogenesis of SLE. Journal of Biomedicine and Biotechnology. http://doi.org/10.1155/2012/347141

Tapper, H., Furuya, W., \& Grinstein, S. (2002). Localized exocytosis of primary (lysosomal) granules during phagocytosis: role of Ca2+-dependent tyrosine 
phosphorylation and microtubules. Journal of Immunology (Baltimore, Md. : 1950), 168(10), 5287-96.

Teo, Z. P., \& Hughes, D. (2003). The Role of Macrophages in Apoptosis : Initiator, Regulator, Scavenger. Reviews in Undergraduate Research, 2, 7-11.

Thompson, E. G., Schaheen, L., Dang, H., \& Fares, H. (2007). Lysosomal trafficking functions of mucolipin-1 in murine macrophages. BMC Cell Biology, 8, 54. http://doi.org/10.1186/1471-2121-8-54

Treusch, S., Knuth, S., Slaugenhaupt, S. a, Goldin, E., Grant, B. D., \& Fares, H. (2004). Caenorhabditis elegans functional orthologue of human protein h-mucolipin-1 is required for lysosome biogenesis. Proceedings of the National Academy of Sciences of the United States of America, 101(13), 4483-4488.

http://doi.org/10.1073/pnas.0400709101

Tsuruta, F., Green, E. M., Rousset, M., \& Dolmetsch, R. E. (2009). PIKfyve regulates CaV1.2 degradation and prevents excitotoxic cell death. The Journal of Cell Biology, 187(2), 279-94. http://doi.org/10.1083/jcb.200903028

Tuosto, L., Capuano, C., Muscolini, M., Santoni, A., \& Galandrini, R. (2015). The multifaceted role of PIP2 in leukocyte biology. Cellular and Molecular Life Sciences : CMLS, 72(23), 4461-74. http://doi.org/10.1007/s00018-015-2013-0

Underhill, D. M., \& Ozinsky, A. (2002). Phagocytosis of microbes: complexity in action. Annual Review of Immunology, 20, 825-52. http://doi.org/10.1146/annurev.immunol.20.103001.114744

Urbé, S., Mills, I. G., Stenmark, H., Kitamura, N., \& Clague, M. J. (2000). Endosomal 
localization and receptor dynamics determine tyrosine phosphorylation of hepatocyte growth factor-regulated tyrosine kinase substrate. Molecular and Cellular Biology, 20(20), 7685-92.

Van Gisbergen, K. P. J. M., Geijtenbeek, T. B. H., \& Van Kooyk, Y. (2005). Close encounters of neutrophils and DCs. Trends in Immunology. http://doi.org/10.1016/j.it.2005.09.007

van Gisbergen, P. A. C., Li, M., Wu, S. Z., \& Bezanilla, M. (2012). Class II formin targeting to the cell cortex by binding $\mathrm{PI}(3,5) \mathrm{P} 2$ is essential for polarized growth. Journal of Cell Biology, 198(2), 235-250. http://doi.org/10.1083/jcb.201112085

Venkatachalam, K., Wong, C., \& Zhu, M. X. (2014). The role of TRPMLs in endolysosomal trafficking and function. Cell Calcium. http://doi.org/10.1016/j.ceca.2014.10.008

Vergne, I., Chua, J., \& Deretic, V. (2003). Tuberculosis toxin blocking phagosome maturation inhibits a novel Ca2+/calmodulin-PI3K hVPS34 cascade. The Journal of Experimental Medicine, 198(4), 653-9. http://doi.org/10.1084/jem.20030527

Vergne, I., Fratti, R. A., Hill, P. J., Chua, J., Belisle, J., \& Deretic, V. (2004). Mycobacterium tuberculosis Phagosome Maturation Arrest : Mycobacterial Phosphatidylinositol Analog Phosphatidylinositol Mannoside Stimulates Early Endosomal Fusion, 15(February), 751-760. http://doi.org/10.1091/mbc.E03

Viaud, J., Lagarrigue, F., Ramel, D., Allart, S., Chicanne, G., Ceccato, L., Gaits-lacovoni, F. (2014). Phosphatidylinositol 5-phosphate regulates invasion through binding and activation of Tiam1. Nature Communications, 5(May), 4080. http://doi.org/10.1038/ncomms5080 
Vieira, O. V., Botelho, R. J., Rameh, L., Brachmann, S. M., Matsuo, T., Davidson, H. W., Grinstein, S. (2001). Distinct roles of class I and class III phosphatidylinositol 3kinases in phagosome formation and maturation. Journal of Cell Biology, 155(1), 19-25. http://doi.org/10.1083/jcb.200107069

Vieira, O. V, Botelho, R. J., \& Grinstein, S. (2002). Phagosome maturation: aging gracefully. The Biochemical Journal, 366(Pt 3), 689-704. http://doi.org/10.1042/BJ20020691

Wang, W., Zhang, X., Gao, Q., \& Xu, H. (2014). TRPML1: an ion channel in the lysosome. Handbook of Experimental Pharmacology, 222, 631-45. http://doi.org/10.1007/978-3-642-54215-2_24

Welch, H. C. E., Condliffe, A. M., Milne, L. J., Ferguson, G. J., Hill, K., Webb, L. M. C., Stephens, L. R. (2005). P-Rex1 Regulates Neutrophil Function. Current Biology (Vol. 15). http://doi.org/10.1016/j.cub.2005.09.050

Wick, M. J. (2003). The role of dendritic cells in the immune response to Salmonella. Immunology Letters, 85(2), 99-102. http://doi.org/DOI: 10.1016/S01652478(02)00230-4

Wong, C. O., Li, R., Montell, C., \& Venkatachalam, K. (2012). Drosophila TRPML is required for TORC1 activation. Current Biology, 22(17), 1616-1621. http://doi.org/10.1016/j.cub.2012.06.055

Xu, Y., Hortsman, H., Seet, L., Wong, S. H., \& Hong, W. (2001). SNX3 regulates endosomal function through its PX-domain-mediated interaction with PtdIns(3)P. Nature Cell Biology, 3(7), 658-66. http://doi.org/10.1038/35083051 
Yu, L., McPhee, C. K., Zheng, L., Mardones, G. A., Rong, Y., Peng, J., Lenardo, M. J. (2010). Termination of autophagy and reformation of lysosomes regulated by mTOR. Nature, 465(7300), 942-6. http://doi.org/10.1038/nature09076

Zeevi, D. A., Frumkin, A., \& Bach, G. (2007). TRPML and lysosomal function. Biochimica et Biophysica Acta, 1772(8), 851-8. http://doi.org/10.1016/j.bbadis.2007.01.004

Zhang, J.-M., \& An, J. (2007). Cytokines, Inflammation and Pain. Int Anesthesiol Clin., 45(2), 27-37. http://doi.org/10.1097/AIA.0b013e318034194e.

Zhang, Y., Zolov, S. N., Chow, C. Y., Slutsky, S. G., Richardson, S. C., Piper, R. C., Weisman, L. S. (2007). Loss of Vac14, a regulator of the signaling lipid phosphatidylinositol 3,5-bisphosphate, results in neurodegeneration in mice. Proceedings of the National Academy of Sciences of the United States of America, 104(44), 17518-23. http://doi.org/10.1073/pnas.0702275104

Zolov, S. N., Bridges, D., Zhang, Y., Lee, W.-W., Riehle, E., Verma, R., Weisman, L. S. (2012). In vivo, Pikfyve generates PI(3,5)P2, which serves as both a signaling lipid and the major precursor for PI5P. Proceedings of the National Academy of Sciences of the United States of America, 109(43), 17472-7. http://doi.org/10.1073/pnas.1203106109

Zoncu, R., Bar-Peled, L., Efeyan, A., Wang, S., Sancak, Y., Sabatini, D. M., ... Steinberg, B. E. (2011). mTORC1 senses lysosomal amino acids through an inside-out mechanism that requires the vacuolar H(+)-ATPase. Science (New York, N.Y.), 334(6056), 67883. http://doi.org/10.1126/science.1207056 


\section{Chapter 2}

\subsection{PIKfyve inhibition interferes with phagosome and endosome maturation in macrophages}

\subsubsection{Contribution of Authors and Co-Authors}

Author: Roya Monica Dayam

contributions: Performed the experiments in figures 1, 2, 3, 4, 5, 7(C, D), 8(E, F, G), and $\mathrm{S} 1$, analyzed data, generated figures and helped in the preparation of the manuscript.

Author: Grace Kim

contributions: performed the experiments in figures $6,7(A, B), 8(C, D)$ and S2.

Co-authors: Akriti Prashar and Mauricio Terebiznik

Contributions: performed the experiment in figure $8(A, B)$

Co-Author: Roberto Botelho

Contributions: Provided essential insight of the experiments and interpretation of the results. Wrote the manuscript.

Status of the manuscript:

Prepared for submission to a peer-reviewed journal

Officially submitted to a peer-reviewed journal

Accepted by a peer-reviewed journal

_ $\mathrm{X}$ _ Published in a peer-reviewed journal

Published by John Wiley \& Sons Ltd

In Volume 15, issue 10, 1143-1163 (2014) 


\title{
PIKfyve inhibition interferes with phagosome and endosome maturation in macrophages
}

The following work has been submitted and published in Traffic journal

\author{
Grace H.E. Kim ${ }^{1 \#}$, Roya M. Dayam ${ }^{1 \#}$, Akriti Prashar ${ }^{2}$, Mauricio Terebiznik ${ }^{2}$ and Roberto J. \\ Botelho $^{1 *}$ \\ ${ }^{1}$ Dept. of Chemistry and Biology and the Molecular Science Program, Ryerson University, \\ Toronto, Ontario, Canada, M5B2K3 \\ ${ }^{2}$ Dept. of Cell and Systems Biology, University of Toronto at Scarborough, Toronto, \\ Ontario, Canada, M1C 1A4 \\ \#These authors contributed equally \\ ${ }^{*}$ Corresponding author: Roberto J. Botelho
}




\subsection{Abstract}

Macrophages eliminate pathogens and cell debris through phagocytosis, a process by which particulate matter is engulfed and sequestered into a phagosome. Nascent phagosomes are innocuous organelles resembling the plasma membrane. However, through a maturation process, phagosomes are quickly remodeled by fusion with endosomes and lysosomes to form the phagolysosome. Phagolysosomes are highly acidic and degradative leading to particle decomposition. Phagosome maturation is intimately dependent on the endosomal pathway, during which diverse cargoes are sorted for recycling to the plasma membrane or for degradation in lysosomes. Not surprisingly, various regulators of the endosomal pathway are also required for phagosome maturation, including phosphatidylinositol-3-phosphate, an early endosomal regulator. However, phosphatidylinositol-3-phosphate can be modified by the lipid kinase PIKfyve into phosphatidylinositol-3,5-bisphosphate, which controls late endosome/lysosome functions. The role of phosphatidylinositol-3,5-bisphosphate in macrophages and phagosome maturation remains basically unexplored. Using Fc $\gamma$ receptor-mediated phagocytosis as a model, we describe our research showing that inhibition of PIKfyve hindered certain steps of phagosome maturation. In particular, PIKfyve antagonists delayed removal of phosphatidylinositol-3-phosphate and reduced acquisition of LAMP1 and cathepsin D, both common lysosomal proteins. Consistent with this, the degradative capacity of phagosomes was reduced but phagosomes appeared to still acidify. We also showed that trafficking to lysosomes and their degradative capacity was reduced by PIKfyve inhibition. Overall, we provide evidence 
that PIKfyve, likely through phosphatidylinositol-3,5-bisphosphate synthesis, plays a significant role in endolysosomal and phagosome maturation in macrophages.

\subsection{Introduction}

Phosphoinositide (PI) lipids are architects of organelle identity. There are seven PI species, each possessing a unique set of effector proteins and subcellular distribution. In this way, they help decorate a host organelle membrane with cognate effectors that then endow that organelle with its properties (1-7).

Pls play an important role in governing the endosomal pathway, which is composed of a highly dynamic and heterogeneous population of endosomes and lysosomes (8-10). Early endosomes receive and sort endocytic and biosynthetic cargo molecules for recycling to the plasma membrane or for degradation in lysosomes (10-13). Once recyclable cargo molecules are removed, early endosomes are thought to mature into multivesicular bodies $(11,12)$. This requires the ESCRT machinery that sorts ubiquitylated-membrane proteins into intraluminal vesicles $(11,14-16)$. Multivesicular bodies then fuse with the late endosomes, which then merge with lysosomes to elicit full molecular degradation (11). Most of these endosomal processes are regulated by two PIs.

Phosphatidylinositol-3-phosphate [Ptdlns(3)P] is thought to be enriched on early endosomes (17). Typically, Ptdlns(3)P binds to FYVE and PX-domain containing protein effectors (18-21). For example, PtdIns(3)P recruits the FYVE-domain containing proteins 
EEA1 and Hrs to early endosomes, which respectively directs homotypic endosome fusion and ESCRT-dependent sorting of cargo for degradation (22-25).

In contrast to Ptdlns(3)P, phosphatidylinositol-3,5-bisphosphate $\mathrm{PI}(3,5) \mathrm{P} 2$ is thought to predominate in late endosomes and/or lysosomes (26-29). In mammals, PI(3,5)P2 is synthesized by phosphorylation of PtdIns(3)P by PIKfyve, a Ptdlns(3)P 5-kinase (30, 31). Inhibition of $\mathrm{PI}(3,5) \mathrm{P} 2$ synthesis leads to several defects including a dramatic enlargement of late endosomes/lysosomes, reduction in late endosomal/lysosomal membrane recycling and an apparent accumulation of autophagosomes (27, 32-35). However, it remains unclear how $\mathrm{PI}(3,5) \mathrm{P} 2$ performs its functions since there are only a few known PI(3,5)P2 effectors. In mammals, these include TRPML-1, a lysosomal $\mathrm{Ca}^{2+}$ channel, and Raptor, a component of the mTORC1 complex that manages the metabolic state of cells $(36,37)$.

Recently, there have been a number of studies linking PIKfyve and/or PI(3,5)P2 to neuronal function and neurodegeneration (35, 38-41). By comparison, there have been few studies examining their role in immune function. For instance, macrophages are immune cells that hunt and kill pathogens by phagocytosis (42-44). During phagocytosis, pathogens are engulfed into a plasma membrane-derived phagosome. Phagosomes then undergo a maturation process by sequentially fusing with endosomes and lysosomes forming phagolysosomes $(43,45,46)$. Phagolysosomes are highly acidic and proteolytic, leading to pathogen degradation $(43,47,48)$.

Phagosome maturation is intertwined with the endolysosome membrane system. Indeed, PtdIns(3)P is necessary for phagosome maturation $(49,50)$. First, phagosomes 
transiently acquire Ptdlns(3)P soon after completion of phagocytosis as detected by FYVE-GFP or PX-GFP chimeric proteins $(49,51)$. Second, hindrance of Ptdlns(3)P synthesis blocks phagosomal acquisition of lysosomal markers (49-51). However, little has been done to examine the role of $\mathrm{PI}(3,5) \mathrm{P} 2$ in phagosome maturation. It has been suggested that shRNA-mediated silencing of PIKfyve or treatment of macrophages with YM201636, an antagonist of PIKfyve (52), delays the loss of Ptdlns(3)P from phagosomes (53). Additionally, treatment of macrophages with YM201636 reduced the ability of Salmonella to survive within macrophages, implying that PIKfyve may modulate the maturation of Salmonella-containing phagosomes (54). Moreover, PIKfyve may regulate TLR9 trafficking and signaling in response to CpG oligodeoxynucleotides in macrophages (55). Aside from these few studies, the role of PI(3,5)P2 in macrophage membrane trafficking is poorly studied. In fact, given that Ptdlns(3)P is a precursor for $\mathrm{PI}(3,5) \mathrm{P} 2$, inhibition of the former likely reduces the latter. Thus, it is unclear what specific role both PIs play in phagosome maturation.

Here, we studied the role of PIKfyve in phagosome maturation by treating the RAW 264.1 (RAW) macrophage model cell line with various antagonists of PIKfyve $(34,52$, 56), and using siRNA-mediated repression of PIKfyve expression. We find that PIKfyve inhibition interferes with various steps of phagosome maturation and trafficking of immune complexes mediated by Fcy receptors. 


\subsection{Results and Discussion}

\subsubsection{Inhibition of PIKfyve in macrophages}

PIKfyve can be inhibited in various ways: expression of dominant-negative mutants (27), gene silencing $(33,57)$ or by pharmacological antagonists $(34,52,56)$. Although molecular methods like siRNA can be highly specific at the gene level, these methods usually take days to take effect, leading to potentially chronic, indirect effects of PIKfyve inhibition and endolysosome swelling. By contrast, pharmacological methods, although possessing a risk of off-target effects, are ideal to acutely block PIKfyve activity and examine its direct and indirect roles. Therefore, we first compared the potency of PIKfyve gene silencing and antagonists in macrophages. Since vacuolation is the quintessential hallmark of PIKfyve inhibition, we quantified vacuolation as a proxy for PIKfyve inactivation - with the caveat that this is not a direct measure of PIKfyve activity.

We tested three different inhibitors of PIKfyve that are now available: YM201636 is the most commonly utilized PIKfyve antagonist $(35,52,58,59)$; MF4 is chemically related to YM201636, differing by an amino group on the pyridine ring (34); and apilimod, a recently discovered PIKfyve antagonist that was previously evaluated in clinical trials to treat Crohn's disease and rheumatoid arthritis (56). First, we examined macrophage vacuolation as a function of drug concentration in order to identify comparable effective concentrations for each drug. Cells were treated for $2 \mathrm{~h}$ with various drug concentrations followed by live-cell imaging. As illustrated in Figure 2.1A, cells were predominantly devoid of vacuoles when untreated. However, treatment of cells with 
progressively larger doses of each drug led to greater number of and increasingly larger vacuoles (Fig. 2.1A shows apilimod-treated cells but similar results were obtained for YM201636 and MF4, not shown). To facilitate quantification, we then scored for the number of vacuoles $>1 \mu \mathrm{m}$ in diameter per cell for each drug treatment (Fig. 2.1B). Consistent with the visual inspection, vacuolation increased proportionally with drug concentration. Using this, we defined concentrations for each drug that resulted in intermediate-level vacuolation $(<8$ vacuoles/cell) versus high-level vacuolation ( $>8$ vacuoles/cell). Using concentrations of drugs that produced intermediate-level vacuolation after $2 \mathrm{~h}$ of exposure, we then showed that vacuolation was also time dependent; longer exposure to each drug amplified macrophage vacuolation (Fig. 2.1C). Overall, we could define conditions for each drug that led to intermediate or high levels of vacuolation to identify comparable effective concentrations for each drug with likely comparable levels of PIKfyve inhibition.

To complement the pharmacological inactivation of PIKfyve, we also tested silencing of PIKfyve in RAW cells by electroporation of SMARTpool oligonucleotides against PIKfyve $\left(\operatorname{siRNA}^{\mathrm{PIK}}\right)$. Cells electroporated with siRNA ${ }^{\mathrm{PIK}}$, but not with scrambled siRNA oligonucleotides, developed small vacuoles, suggesting that PIKfyve expression was at least partially repressed (Fig. 2.1D). However, despite trying various conditions, we could not force more efficient vacuolation by siRNA ${ }^{\mathrm{PIK}}$ in macrophages. It is noteworthy that others have noted difficulty in inducing large vacuoles using siRNA against PIKfyve, likely because small levels of $\mathrm{PI}(3,5) \mathrm{P} 2$ may suffice to prevent formation of large vacuoles $(33,34,60)$. Overall, these experiments suggest that both gene silencing and 
antagonists may be used as complementary approaches to block PIKfyve activity in macrophages.
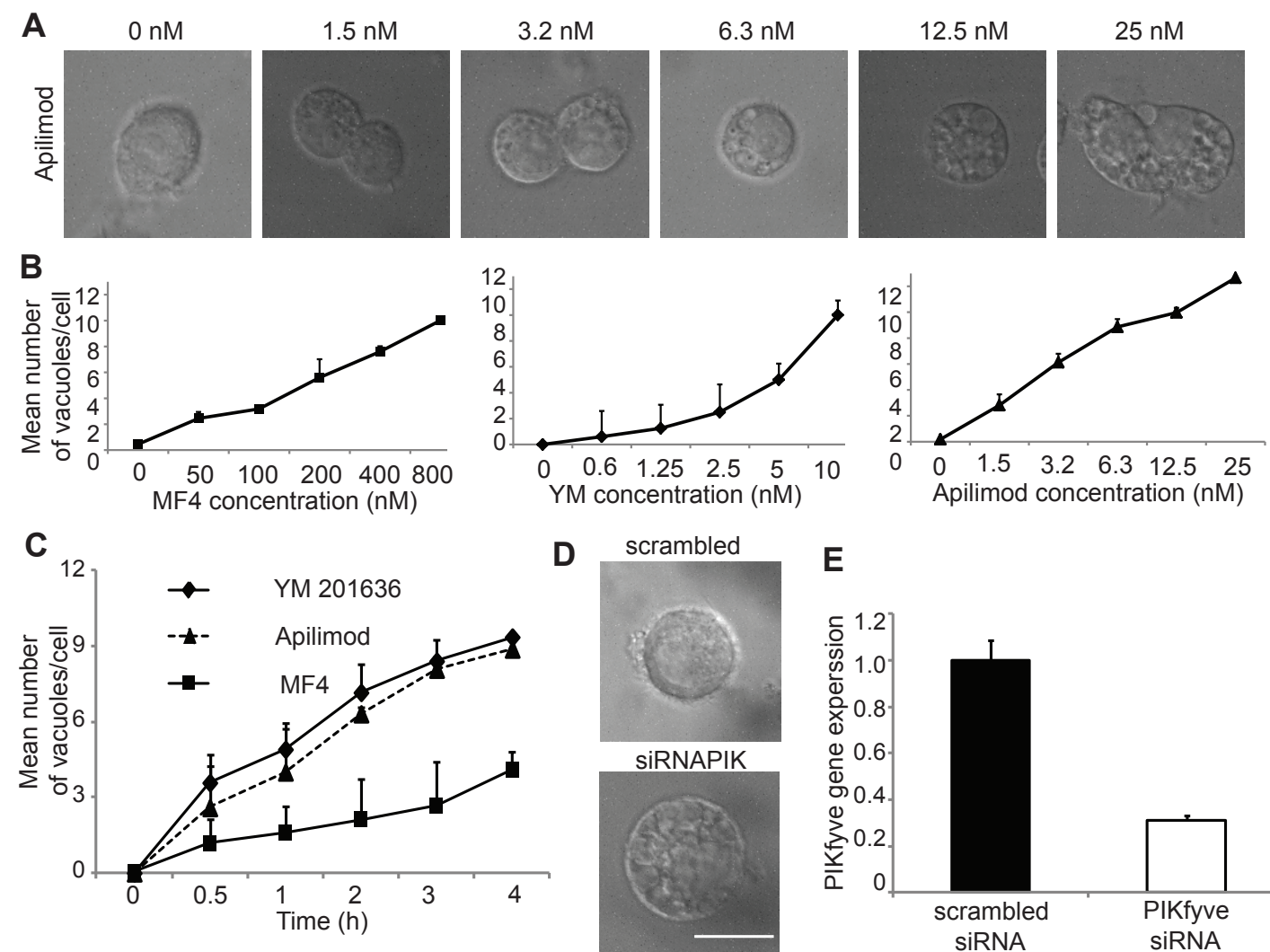

E

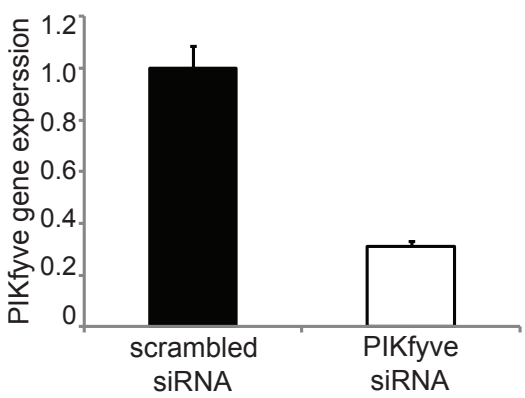

Figure 2.1: Inhibition of PIKfyve and vacuolation in macrophages

A. RAW cells were treated with DMSO $(0 \mathrm{nM})$ or various concentrations of apilimod for $2 \mathrm{~h}$ as indicated. Cells were then imaged live by DIC. We observed a proportional increase in the number and size of vacuoles with increasing concentration of apilimod. B. The number of vacuoles $>1 \mu \mathrm{m}$ in diameter were counted as a function of concentration for apilimod, YM201636 and MF4 after incubation for $2 \mathrm{~h}$. The average number of vacuoles and standard deviation from three experiments are shown. Using one-way ANOVA test, there was a significant difference in vacuole number between concentrations employed ( $p<0.0001$ for apilimod and YM201636 and $p<0.001$ for MF4). C. RAW cells were incubated with drug concentrations that produced intermediatelevels of vacuolation in B for different periods of time (shown are experiments for $6 \mathrm{nM}$ for apilimod, $1.5 \mathrm{nM}$ YM201636 and $100 \mathrm{nM}$ for MF4). The number of vacuoles with diameter $>1 \mu \mathrm{m}$ was also quantified for each drug. We observed a time-dependent amplification in vacuolation for each drug. Using one-way ANOVA test, there was a significant difference in vacuole number between the time points for each drug 
( $p<0.0001$ for apilimod and YM201636 and $p<0.05$ for MF4). D. RAW macrophages were electroporated with scrambled or PIKfyve siRNA oligonucleotides and observed live by DIC. We observed that siRNA ${ }^{\text {PIK }}$-treated cells developed many small vacuoles, unlike scrambled oligonucleotides. Scale bar $=10 \mu \mathrm{m}$.

\subsubsection{Differential effects on early and late endocytic organelles in PIKfyve} inhibited macrophages

PIKfyve inhibition typically swells late endocytic organelles like late endosomes and Iysosomes $(26,27,29)$. However, there are also reports showing that PIKfyve inactivation can enlarge early endosomes $(33,35,61)$. Since this apparent discrepancy may be due to physiological differences among cell types and/or experimental methods, we decided to examine which endocytic organelles were susceptible to vacuolation in PIKfyve-blocked macrophages.

To identify the nature of the vacuoles, we assessed if they were early endosome-like and/or late endosome/lysosome-like by respectively staining against endogenous EEA1 and LAMP1 $(24,49)$. Importantly, we also assessed whether EEA1 and/or LAMP1 staining differed between cells with intermediate and high levels of vacuolation by controlling the concentration of each drug. As expected, all three drugs caused swelling of LAMP1-positive structures, irrespective of whether conditions led to intermediate or extensive vacuolation, indicating that the morphology of late endosomal/lysosomes is very sensitive to PIKfyve inhibition (Fig. 2.2). However, there was a disparate effect on EEA1-positive compartments. In cells with intermediate vacuolation, but which nevertheless exhibited swollen LAMP1-positive compartments, EEA1 was predominantly limited to punctate structures (Fig. 2.2A). By contrast, cells that suffered high-levels of 
vacuolation also displayed EEA1-positive vacuoles (Fig. 2.2B). This was true for MF4, YM201636 and apilimod treatments.

In comparison, we observed that siRNA ${ }^{\text {PIK }}$ led to many LAMP1-stained "doughnuts", while EEA1 remained punctate, similarly to cells electroporated with non-targeting siRNA (Fig. 2.2C). Thus, the effect on endosomes and lysosomes by siRNA ${ }^{\mathrm{PIK}}$ treatment best resembles drug treatments that led to intermediate vacuolation. This is consistent with the mild-vacuolation phenotype observed in cells treated with siRNA ${ }^{\mathrm{PIK}}$ (Fig. 2.1D). Overall, these experiments suggest that late endosomes/lysosomes are more susceptible to swelling than early endosomes in response to PIKfyve inactivation. We speculate that either small levels of $\mathrm{PI}(3,5) \mathrm{P} 2$ are sufficient to maintain early endosome morphology or that swelling occurs by an indirect mechanism. For example, enlargement of EEA1-positive structures may result from a buildup of PtdIns(3)P shown to occur during PIKfyve inhibition $(56,61,62)$, or alternatively, due to altered membrane trafficking like impaired recycling $(33,34)$. 


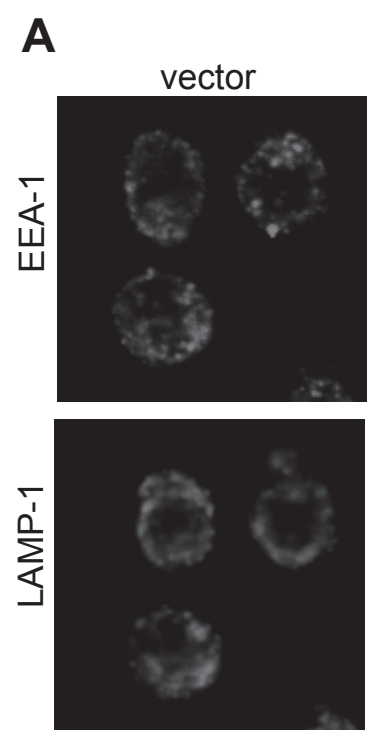

Apilimod

YM

(0.6 nM)
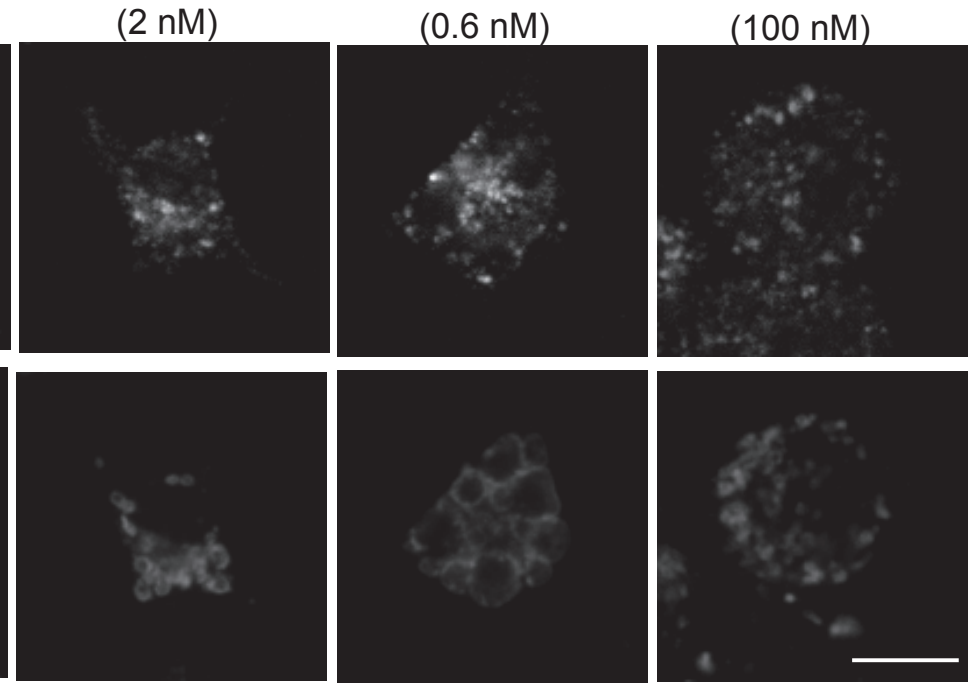

B

Apilimod

(25 nM)
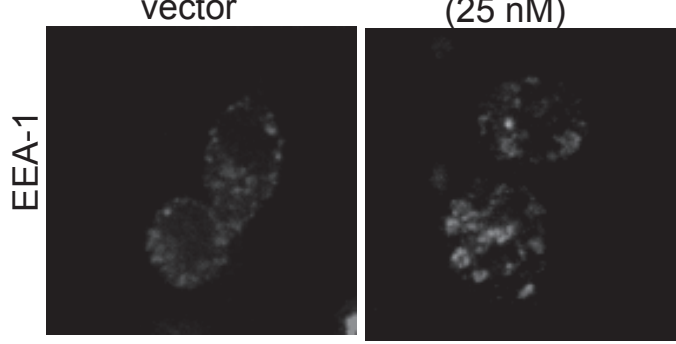

YM

(10 nM)

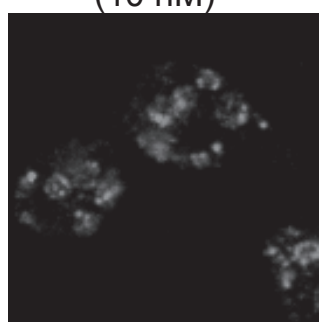

MF4

$(800 \mathrm{nM})$
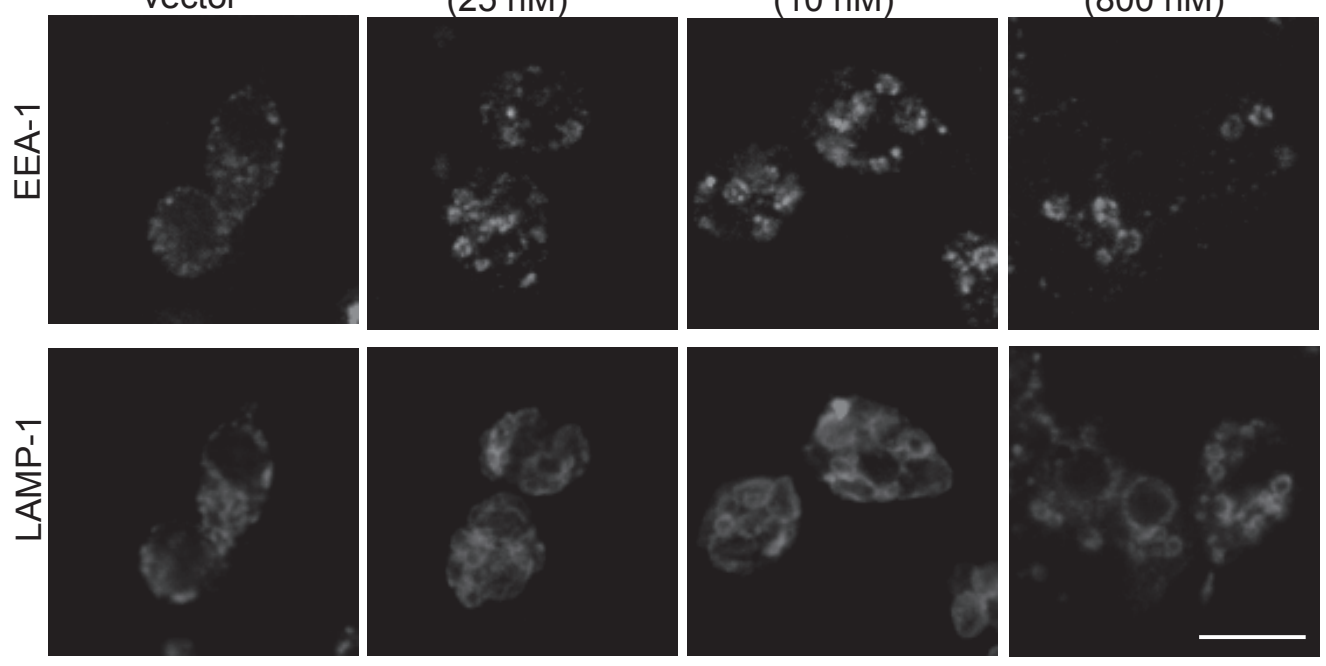

C

Scrambled-siRNA
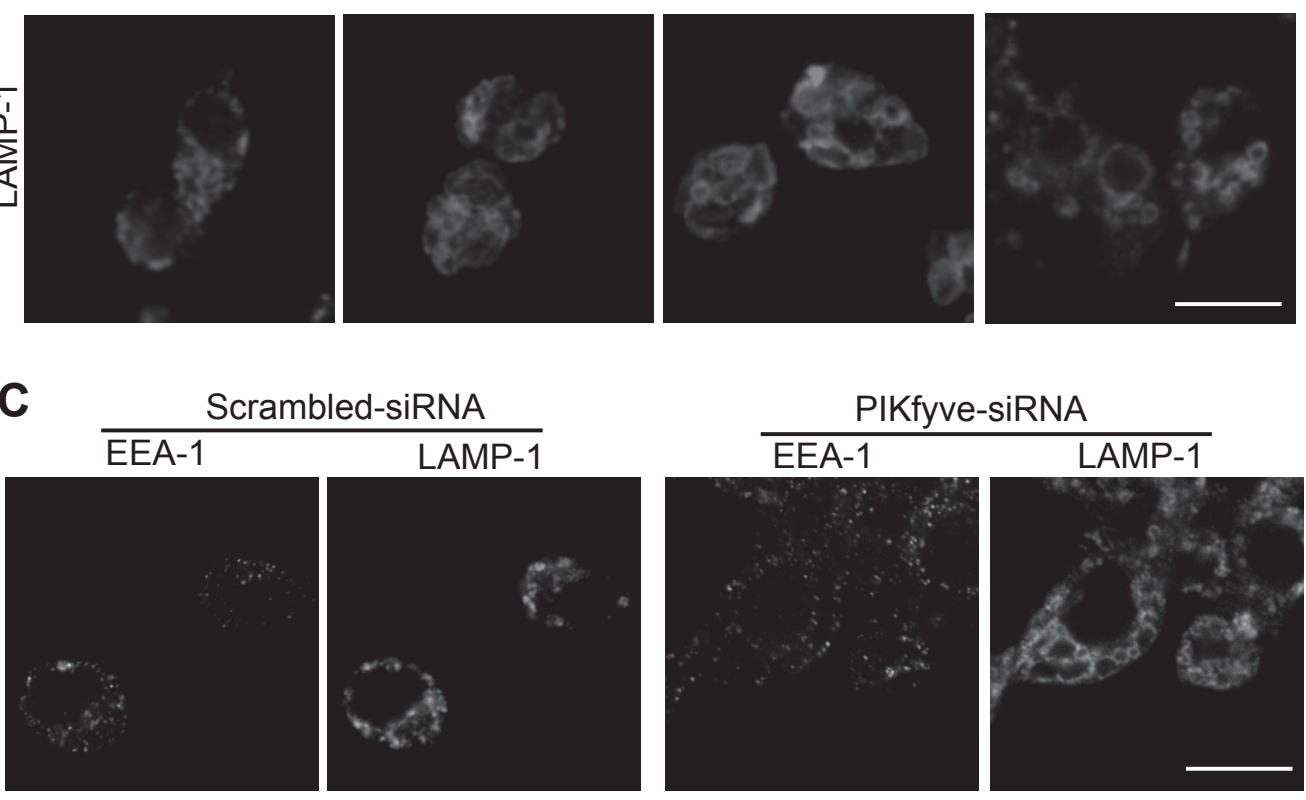
Figure 2.2: The identity of vacuoles in PIKfyve-inhibited macrophages.

A. RAW macrophages were exposed for $2 \mathrm{~h}$ to $0.6 \mathrm{nM}$ YM201636, $2 \mathrm{nM}$ apilimod or 100 $\mathrm{nM}$ MF4 to induce intermediate levels of vacuolation. B. RAW macrophages were exposed to $10 \mathrm{nM}$ YM201636, $25 \mathrm{nM}$ apilimod or $800 \mathrm{nM}$ MF4 for $2 \mathrm{~h}$ to induce highvacuolation. C. RAW macrophages were electroporated with scrambled or PIKfyve siRNA oligonucleotides. In all cases, cells were then processed for coimmunofluorescence staining against endogenous EEA1 and LAMP1. In all conditions, except for control, LAMP1 compartments were vacuolated to various degrees. EEA1 compartments appeared punctate in vector-treated cells, drug conditions that led to intermediate vacuolation $(A)$ and in SiRNA ${ }^{\text {PIK }}$-treated cells $(C)$. However, conditions that led to high-vacuolation caused EEA1-stained vacuoles to appear (B). Scale bar $=10 \mu \mathrm{m}$.

\subsubsection{PIKfyve activity is required for phagocytosis}

Macrophages roam through tissues to dispose of pathogens by phagocytosis (49). We speculated that PIKfyve might be necessary to maintain macrophage phagocytic proficiency because there are several precedents showing that PIKfyve can affect plasma membrane-associated processes (63). For example, neurons silenced for PIKfyve suffer reduced endocytosis of $\mathrm{Ca}_{\mathrm{v}} 1.2$ in response to glutamate signaling, leading to neuronal excitotoxic death (38). In another example, PIKfyve overexpression enhances surface expression of various channels and transporters like SLC6A19, an amino acid transporter, and an inward rectifying $\mathrm{K}^{+}$channel, Kir2.1 $(64,65)$. Lastly, PIKfyve and myotubularins cooperate to control cell migration during wound-healing (60). Hence, we inspected the capacity of PIKfyve-halted macrophages to undertake phagocytosis. We used Fcy receptor-mediated uptake of IgG-opsonized particles as a model since this is one of the best characterized forms of phagocytosis (66).

To better understand the importance of PIKfyve activity in phagocytosis, we compared the phagocytic index in cells subjected to various concentrations of apilimod, 
MF4 and YM201636. As shown in Figure 2.3A, lower-doses of each drug (up to $200 \mathrm{nM}$ MF4, up to $10 \mathrm{nM}$ YM201636 and up to $25 \mathrm{nM}$ apilimod) had little effect on the phagocytic index, despite causing at least intermediate levels of vacuolation. However, concentrations that surpassed the concentrations indicated above significantly attenuated phagocytosis (Fig. 2.3A). Similarly, pro-longed exposure of cells to PIKfyve inhibitors ( 6 h) hindered phagocytic uptake relative to short exposures ( $2 \mathrm{~h}$; Fig. 3B). To complement the pharmacological-based experiments, we also observed a significant reduction of $33 \pm 7 \%$ in the phagocytic index of cells silenced for PIKfyve (siRNA ${ }^{\mathrm{PIK}}$ ) relative to cells electroporated with the non-targeting oligonucleotides (Fig. 2.3C).

Taken together, our data suggests that PIKfyve activity is required for Fcymediated phagocytosis of IgG particles. Although the exact role for PIKfyve in particle engulfment is not known, our observations are consistent with the following possible models: i) PIKfyve may play a direct role in phagocytosis, requiring low levels of activity to maintain uptake - this would be consistent with nearly intact phagocytosis by cells exposed to lower, but effective doses of PIKfyve inhibitors, but significantly reduced uptake at higher-doses of the antagonists; ii) and/or alternatively, PIKfyve may play an indirect role in particle uptake. Possibly, this indirect role may be due to distortion of either membrane recycling, exocytosis, and/or signaling processes that can occur during protracted loss of PIKfyve $(33,52,60,67-69)$. Interestingly, it was recently shown that TRPML1, a PI(3,5)P2 -dependent $\mathrm{Ca}^{+2}$ channel found on lysosomes (37), is required for phagocytosis of large particles (70). It is postulated that TRPML1 is necessary for lysosome exocytosis that aids in the uptake of large particles (60). Our work shows that 
even small particles are eventually affected by PIKfyve suppression. While it is possible that this is partially due to suppression of localized exocytosis, it is tempting to speculate that PIKfyve may be required for phagocytosis by a similar mechanism that it is required for cell migration - by acting in concert with myotubularins to generate PtdIns(5)P (60). In addition, it will be interesting to expand this study to other phagocytic receptors like complement receptor-mediated phagocytosis.
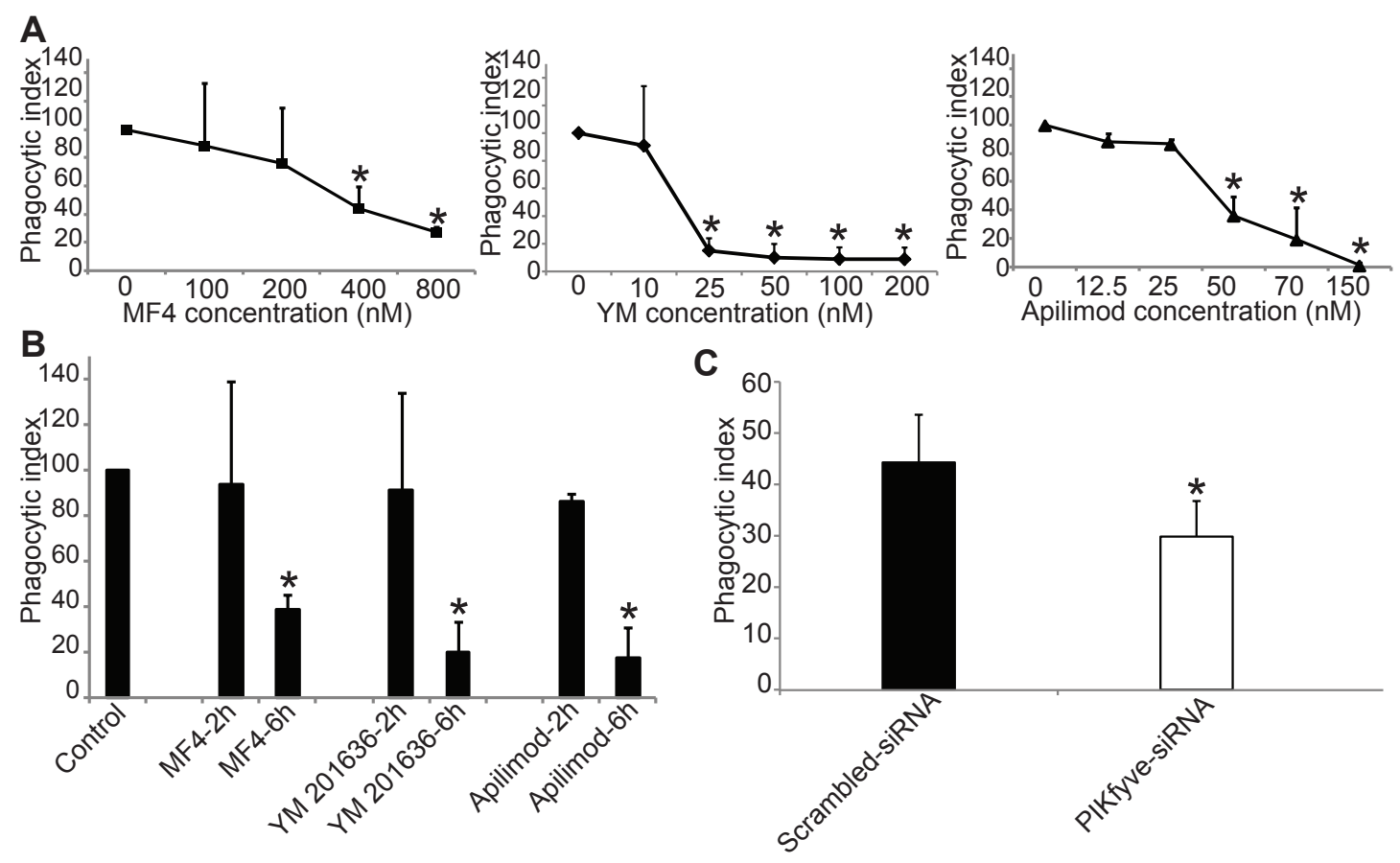

Figure 2.3: The effect of PIKfyve antagonists on phagocytosis in macrophages.

A. RAW macrophages were pre-treated with either vector $(0 \mathrm{nM})$ or with the indicated concentrations of MF4, YM201636 or apilimod for $2 \mathrm{~h}$. Cells were then permitted to phagocytose IgG-opsonized beads for $15 \mathrm{~min}$, fixed and stained with fluorescent antihuman IgG antibodies to identify external beads. B. RAW macrophages were pretreated with vector (control), $200 \mathrm{nM}$ MF4, $10 \mathrm{nM}$ YM201636 or $25 \mathrm{nM}$ apilimod for either $2 \mathrm{~h}$ or $6 \mathrm{~h}$ and then allowed to perform phagocytosis. C. RAW macrophages were electroporated with scrambled or siRNA ${ }^{\mathrm{PIK}}$ oligonucleotides and then permitted to internalize IgG-opsonized beads as described above. In all cases, the phagocytic index was then quantified as the number of phagosomes per 100 cells and normalized to the respective controls. For each case, shown is the mean phagocytic index and standard deviation from at least three independent experiments and from at least 100 cells 
counted per condition. In $\mathrm{A}, *$ denotes significant differences in phagocytic index between control and concentrations of each drug identified by using the one-way ANOVA test followed by Tukey's post-hoc test $(p<0.01)$; In $B$, * denotes significant differences in phagocytic index between control and $6 \mathrm{~h}$ treatments $(p<0.01)$ and between $2 \mathrm{~h}$ and $6 \mathrm{~h}$ treatments for each respective drug $(p<0.01)$ by using one-way ANOVA, followed by Tukey's post-hoc test; In C, there was a significant different between scrambled and siRNA ${ }^{\text {PIK }}$ samples using Student's t-test $(p<0.05)$.

\subsubsection{Early phagosome maturation is altered in PIKfyve-blocked macrophages}

Immediately after scission, phagosomes undergo a rapid remodeling to convert the phagosomal membrane from a plasma membrane-like state to one similar to early endosomes, acquiring markers like PtdIns $(3) P(43,49,50)$. As noted above, acquirement of Ptdlns(3)P can be probed by expression of 2FYVE-GFP (49). Since PIKfyve converts Ptdlns(3)P to $\mathrm{PI}(3,5) \mathrm{P} 2$, we predicted that blocking PIKfyve might alter the kinetics of phagosome acquisition and/or loss of the 2FYVE-GFP probe.

To do this, we acquired time-series of cells expressing 2FYVE-GFP and undertaking phagocytosis. We tracked the fluorescence intensity of 2FYVE-GFP on phagosomes relative to cytosolic signal in control and $2 \mathrm{~h}$ MF4-treated cells using (Fig. 2.4A). Importantly, we synchronized phagosome maturation relative to the apparent closure of the phagosome (time $=0 \mathrm{~min}$ ). Using this methodology, we determined that there was no apparent difference in the acquisition rates of 2FYVE-GFP between control and MF4-treated cells (Fig. 2.4). In fact, 2FYVE-GFP fluorescence intensity peaked at about 4 min under both conditions. However, the kinetics of 2FYVE-GFP dissociation were significantly slower in MF4-treated cells (Fig. 2.4). In control cells, phagosomes were divested of more than $50 \%$ of the initial 2FYVE-GFP signal by $7 \mathrm{~min}$ and were 
devoid of the GFP probe by 14-16 min (Fig. 2.4B). In comparison, in MF4-treated cells, phagosomes took 13 min to lose $50 \%$ of the initial 2FYVE-GFP intensity and over 20 min for the probe intensity to equal that of the surrounding cytosol (Fig. 2.4B). Overall, this indicates that PIKfyve-suppression altered the kinetics of 2FYVE-GFP dissociation, but not its acquisition. This appears to be consistent with recent work by Hazeki et al., who demonstrated that both PIKfyve and PTEN, a PI 3-phosphatase, both contribute to the elimination of PtdIns(3)P from phagosomes (53).
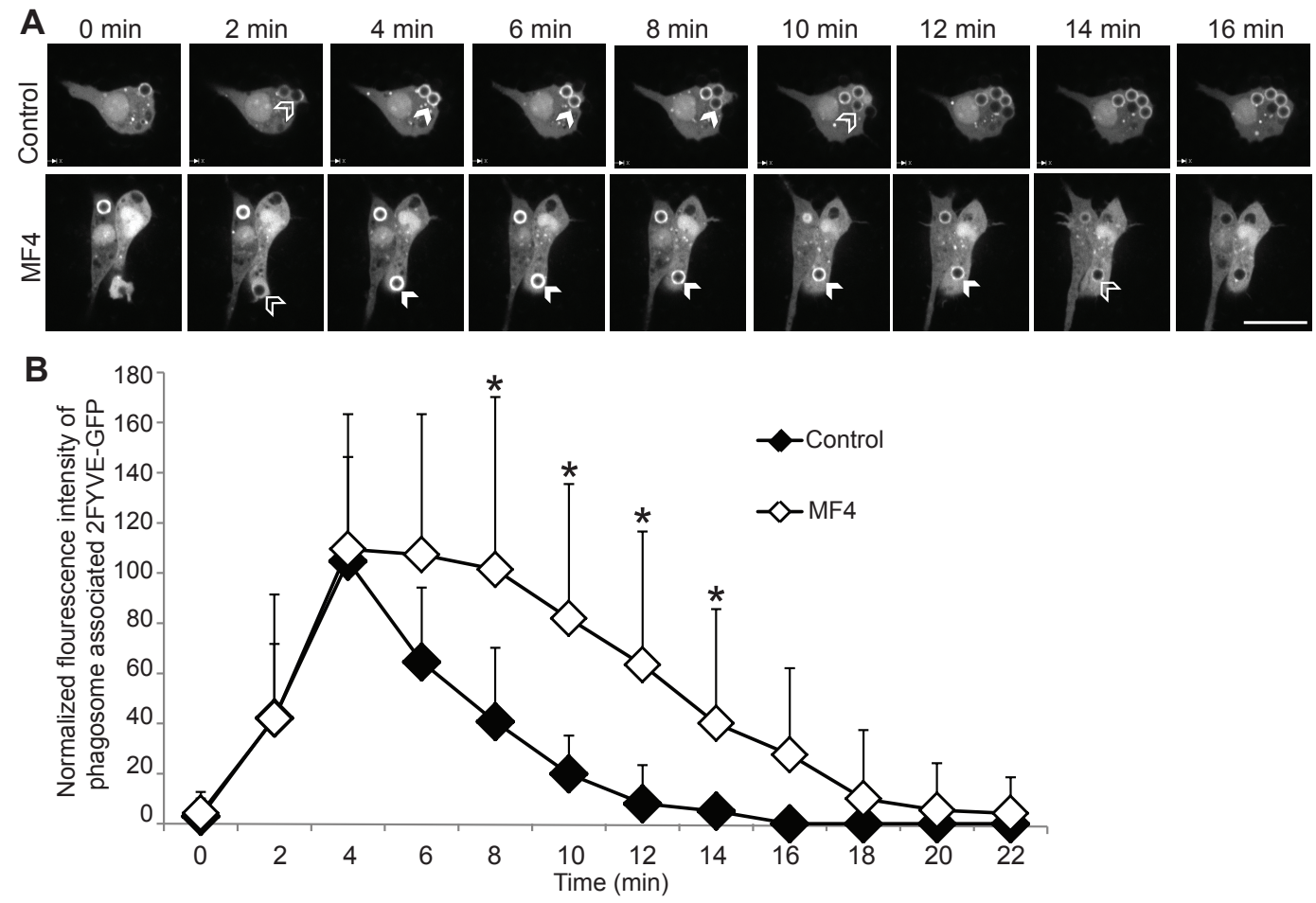

Figure 2.4: PIKfyve inhibition affects the kinetics of Ptdlns(3)P on phagosomes.

RAW cells were transfected with plasmids encoding 2FYVE-GFP to probe for Ptdlns(3)P. Cells were then treated with vector or with $200 \mathrm{nM}$ MF4 for $2 \mathrm{~h}$ and then prepared for live-cell confocal imaging. IgG-opsonized beads were added to cells under observation. A. Representative frames of time-series from control and MF4-treated cells showing the behaviour of 2FYVE-GFP in cells undergoing phagocytosis. For each nascent phagosome, time is calibrated to the apparent phagosome closure (time shown in minutes). Open arrows indicate a "tracked" phagosome with little to no 2FYVE-GFP fluorescence, while closed arrows indicate the same phagosome with 2FYVE-GFP fluorescence. B. Shown is 
the average fluorescence intensity ratio and standard deviation of 2FYVE-GFP on phagosomes to cytosolic signal normalized to control peak fluorescence over every two minutes from apparent phagosome closure. The average is based on at least 12 phagosomes from at least six time-series acquired from at least three independent experiments for each condition. Asterisk $(*)$ indicates a significant difference in fluorescence intensity in phagosome-associated 2FYVE-GFP between control and MF4treated cells at that respective time point using Student's t-test $(p<0.05)$.

2.4.5 PIKfyve activity is required for phagosomes to acquire lysosomal markers The early endosome-nature of phagosomes is short-lived, typically lasting less than 15 minutes (43). Phagosomes then fuse with late endosomes and lysosomes to convert into phagolysosomes (43). While the transient Ptdlns(3)P is necessary for phagolysosome biogenesis, the role of $\mathrm{PI}(3,5) \mathrm{P} 2$ in this process remains to be investigated.

To test the relative importance of Ptdlns(3)P and $\mathrm{PI}(3,5) \mathrm{P} 2$ in phagolysosome formation, we first compared control cells (cells treated with vector), cells pre-treated with 30 min of $100 \mu \mathrm{M}$ LY294002, a PtdIns 3-kinase inhibitor, and cells exposed for $2 \mathrm{~h}$ to $100 \mathrm{nM} \mathrm{MF4,} \mathrm{which} \mathrm{permits} \mathrm{efficient} \mathrm{phagocytosis.} \mathrm{After} \mathrm{the} \mathrm{drug} \mathrm{treatment,} \mathrm{cells}$ were then allowed to engulf IgG-coated beads, followed by a $1 \mathrm{~h}$ chase to elicit maturation and then processed and stained for endogenous LAMP1, a classical marker of late endosomes and lysosomes. To aid in the quantification of the amount of LAMP1 associated with each phagosome, we converted LAMP1 fluorescence intensity to a falsecolour palette and assigned phagosomes into one of three fluorescence intensity groups. Using 8-bit images, the false-colour ranges were white-yellow (grayscale intensities of 255-180), orange-red (grayscale intensities of 180-80) and blue-purple 
colour (grayscale intensities of $80-1$ ), which respectively indicated strong LAMP1 $\left(\right.$ LAMP1 $\left.^{+}\right)$, intermediated LAMP1 (LAMP1 $\left.{ }^{+/}\right)$and weak LAMP1 staining (LAMP1; Fig. 2.5). After scoring at least 65 phagosomes per condition across six experiments for control- and MF4-treatments, and at least 30 phagosomes per experiment across three experiments for LY294002-treatments, we concluded that LY294002- and 2 h MF4treatments significantly reduced the number of phagosomes strongly decorated with LAMP1 relative to control cells (Fig. 2.5A). Specifically, whereas $60 \pm 12 \%$ of phagosomes were $\mathrm{LAMP}^{+}$in control cells, only $25 \pm 17 \%$ and $7 \pm 6 \%$ of phagosomes were LAMP1+ in MF4-treated and LY294002-treated cells, respectively (Fig. 2.5A, 2.4B). Conversely, LY294002-treated cells had significantly more LAMP1' phagosomes $(37 \pm 26 \%)$, relative to MF4-incubated cells $(18 \pm 10 \%)$ and to control cells (3 $\pm 2 \%$; Fig. 2.5A, 2.4B). Importantly, a similar analysis with cells treated with 10 nM YM201636 or 25 nM apilimod for 1-2 h also showed a potent block in phagosome acquisition of LAMP1 (Supplementary Figure S1).

While the 1-2 $\mathrm{h}$ drug-treatments permit one to look at the potential direct role of PIKfyve in phagosome maturation, we also queried the impact of chronic inhibition of PIKfyve on phagosome maturation. First, we found that a $6 \mathrm{~h}$ MF4 incubation more strongly blocked phagosome maturation; the number of $\mathrm{LAMP1}^{+}$phagosomes in cells exposed to $6 \mathrm{~h}$ of MF4 was $15 \pm 7 \%$, which was lower than the $25 \%$ LAMP1 ${ }^{+}$phagosomes found in cells exposed to $2 \mathrm{~h}$ of MF4 (Fig. 2.5B). Second, and consistent with this, electroporation of siRNA ${ }^{\mathrm{PIK}}$ caused a similar decrease in $\mathrm{LAMP1}^{+}$phagosomes, while increasing the number of $\mathrm{LAMP1}^{-}$phagosomes; these cells had only $8 \pm 3 \% \mathrm{LAMP1}^{+}$ 
phagosomes compared to $45 \pm 3 \% \mathrm{LAMP1}^{+}$phagosomes in cells electroporated with nontargeting oligonucleotides (Fig. 2.5C). The converse was true for LAMP1 phagosome staining, i.e., the number of LAMP1 ${ }^{-}$phagosomes increased significantly in siRNA ${ }^{\mathrm{PIK}}{ }_{-}$ treated cells relative to control cells (Fig. 2.5C). Altogether, phagosomes were more likely to have less LAMP1 staining in cells chronically deficient for PIKfyve relative to cells with short-term loss of PIKfyve.

Finally, and to robustly support our microscopy-based analysis, we also quantified phagosome-associated LAMP1 by flow cytometry in control, apilimod, YM201636 or MF4-treated cells using concentrations conducive to efficient particle uptake. This was done by isolating phagosomes on a sucrose-gradient, processing and staining against LAMP1. Importantly, all PIKfyve antagonists significantly reduced the number of phagosomes containing LAMP1 fluorescence intensity above background (Fig. 2.5E, representative experiment for MF4) and the median fluorescence intensity of LAMP1 associated with phagosomes relative to phagosomes from control cells (Fig. 2.5F). Thus, the flow cytometry analysis demonstrates that PIKfyve inhibition attenuated phagosomal acquirement of LAMP1, consistent with our microscopic analysis.

Overall, our research conveys that PIKfyve plays an important role in phagosome maturation. First, since short-term inhibition of PIKfyve led to inefficient phagosome decoration with LAMP1, this suggests that PIKfyve plays a direct role in phagosome maturation. Mechanistically, this may be through a signaling defect; for example, PIKfyve is now linked to the EGFR-Akt pathway to enhance EGFR degradation (69); 
AMPK appears to stimulate PIKfyve to aid in skeletal muscle contraction (71); and PIKfyve is required for mTORC1 targeting and function (36). Alternatively, or in addition, PIKfyve may play a direct function in mediating phagosome-lysosome fusion. For example, PIKfyve, through $\mathrm{PI}(3,5) \mathrm{P} 2$, may gate TRPML-1 to release $\mathrm{Ca}^{2+}$ from lysosomal stores, which is necessary for lysosome fusion $(37,72)$. Second, and in comparison to acute PIKfyve loss, chronic PIKfyve defects likely blocks phagosome maturation indirectly. For example, long-term PIKfyve loss may sufficiently distort the endosomal membrane system by mistargeting proteins necessary for fusion or altering membrane curvature through extensive swelling of lysosomes. In addition, since there is evidence that Fab1, the yeast ortholog of PIKfyve, controls gene expression, one could even speculate that pro-longed loss of PIKfyve may alter the transcriptome and proteome of cells resulting in suppression of phagosome-lysosome fusion (73).

In conclusion, both Ptdlns(3)P and $\mathrm{PI}(3,5) \mathrm{P} 2$ appear to play an important function in phagosome maturation. In fact, while Ptdlns(3)P inhibition had a more pronounced impact in phagosome maturation, this may simply be an additive effect due to the expected concurrent loss of both Ptdlns(3)P and PI(3,5)P2 in LY294002-incubated cells. Given our observations, there will now be a pressing need to identify PI(3,5)P2 effectors that mediate phagosome maturation. As suggested above, TRPML1 is a prime candidate for future research. 
A
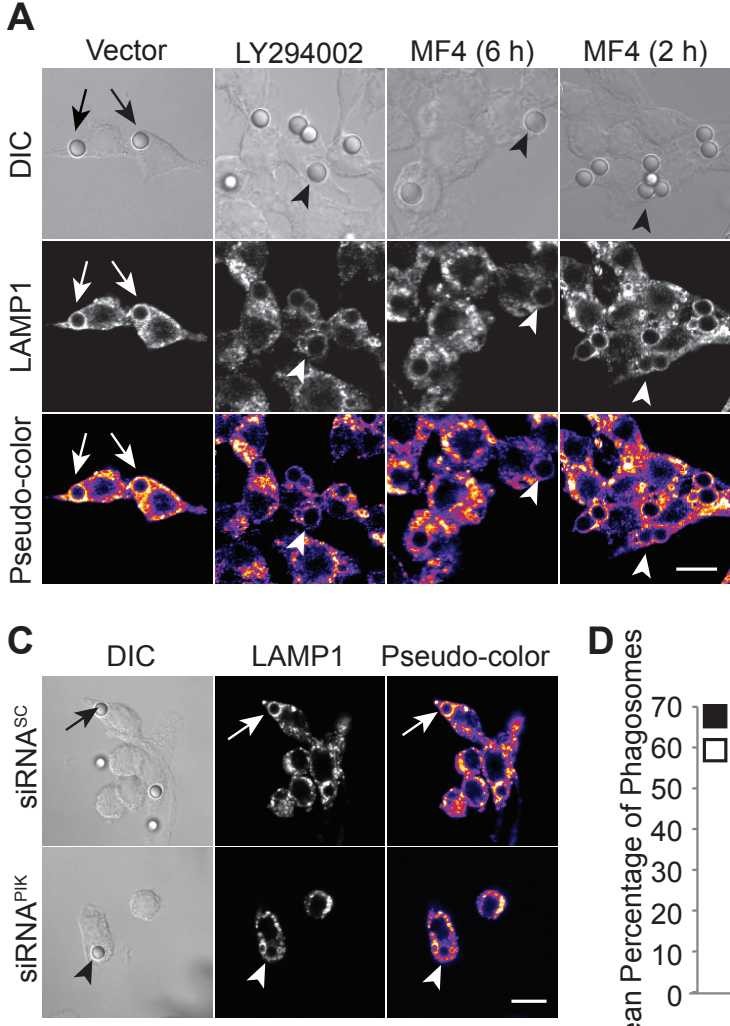

B
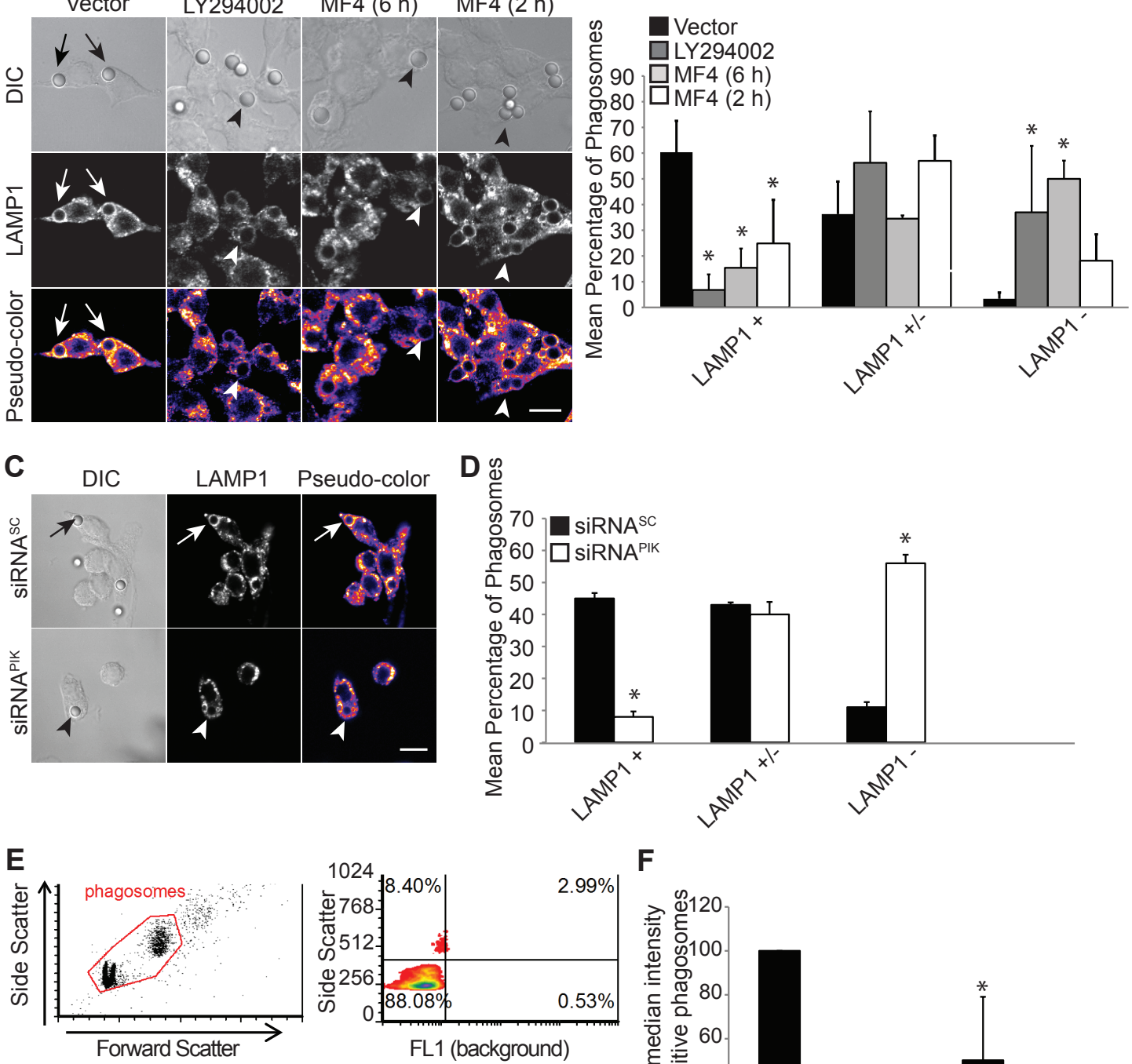

$\mathbf{F}$

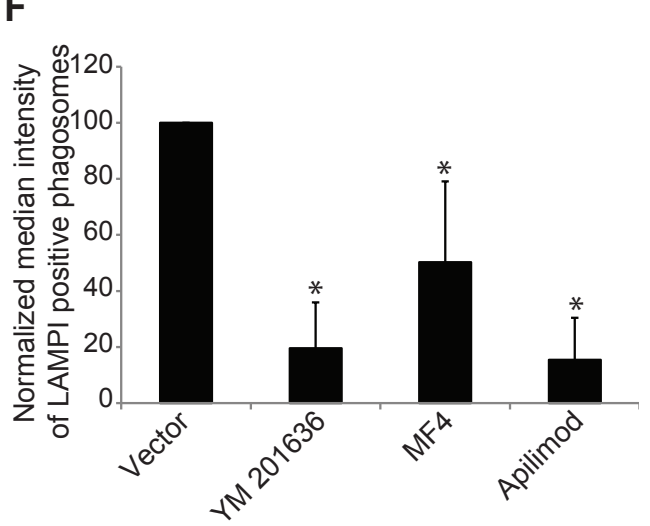

FL1 (vector)

FL1 (MF4)

Figure 2.5: Acute and chronic inhibition of PIKfyve attenuate phagosome acquisition of LAMP1.

A-B. Cells were treated with vector (control) or incubated with $100 \mathrm{nM}$ LY294002 for 30 min, or exposed to $100 \mathrm{nM} \mathrm{MF} 4$ for $2 \mathrm{~h}$ or $6 \mathrm{~h}$. C-D. Cells were either electroporated with scrambled RNA or with siRNA ${ }^{\mathrm{PIK}}$ oligonucleotides as before. A-D: Subsequently, synchronized phagocytosis of IgG-opsonized beads was elicited, followed by 60 min of phagosome maturation. Cells were then fixed and external beads were stained with anti-human IgG, followed by processing for immunostaining LAMP1. After microscopy, 
images were processed with pseudocolour to represent strong LAMP1 acquisition (LAMP1 ${ }^{+}$, white-yellow), intermediate LAMP1 decoration (LAMP1 ${ }^{+/}$, orange-red) and weak LAMP1 staining (LAMP1', blue-purple). B, D: at least 30 phagosomes per experiment were counted for LY294002-treated macrophages across three experiments. For MF4-treated, siRNA-treated and control cells, at least 65 phagosomes per experiment per condition were counted across six experiments. Shown is the mean percent of phagosomes in each LAMP1-staining category \pm standard deviation. E. Flow cytometry of isolated phagosomes stained with anti-LAMP1 antibodies. Top left: shows scatter distribution of phagosomes; Top right: shows scatter and fluorescence distribution of phagosomes stained with secondary only (background). Bottom left: shows scatter and fluorescence distribution of phagosomes from control cells. Bottom right: shows scatter and fluorescence distribution of phagosomes from MF4-treated cells. Percent values are percent of phagosomes in each quadrant, where phagosomes are considered positive for LAMP1 in the two right quadrants. F. Normalized median LAMP1 intensity of phagosomes with above background fluorescence. Using one-way ANOVA test, followed by Tukey's post-hoc test, we could detect significant differences between control and LY294002, $2 \mathrm{~h}$ and $6 \mathrm{~h}$ MF4 treatments for LAMP1+ and LAMP1staining and between $2 \mathrm{~h}$ and $6 \mathrm{~h}$ MF4 treatments for LAMP1- staining (B); between scrambled and siRNA to PIKfyve for LAMP1+ and LAMP1- staining (D); between control (vector) and YM201636, MF4 and apilimod-treated phagosomes (F). For all cases, the pvalue had to be at least $p<0.05$ to be statistically significant.

\subsubsection{PIKfyve is necessary for efficient trafficking of immune complexes to lysosomes}

We also examined if PIKfyve activity was necessary for trafficking of immune complexes

to lysosomes. To test this, we allowed control and MF4-treated cells to internalize soluble aggregated IgG complexes by Fc $\gamma$ receptor-mediated endocytosis and chased for $0,15,30$ and 60 min to allow trafficking of aggregated IgG-FcyR complexes to lysosomes

(74). We then processed cells and stained cells for LAMP1 and human IgG and scored for co-localization using the Pearson's correlation coefficient (Fig. 2.6A). We found that compared to control cells, MF4-treated cells displayed reduced co-localization between aggregated IgG and LAMP1-positive organelles over the various time points observed 
(Fig. 2.6B). This indicates that PIKfyve is necessary for efficient trafficking of immune complexes to lysosomes in macrophages. This is consistent with several studies showing that PIKfyve helps target signaling receptors like EGFR and Met to lysosomes for degradation $(34,69)$.

A

A

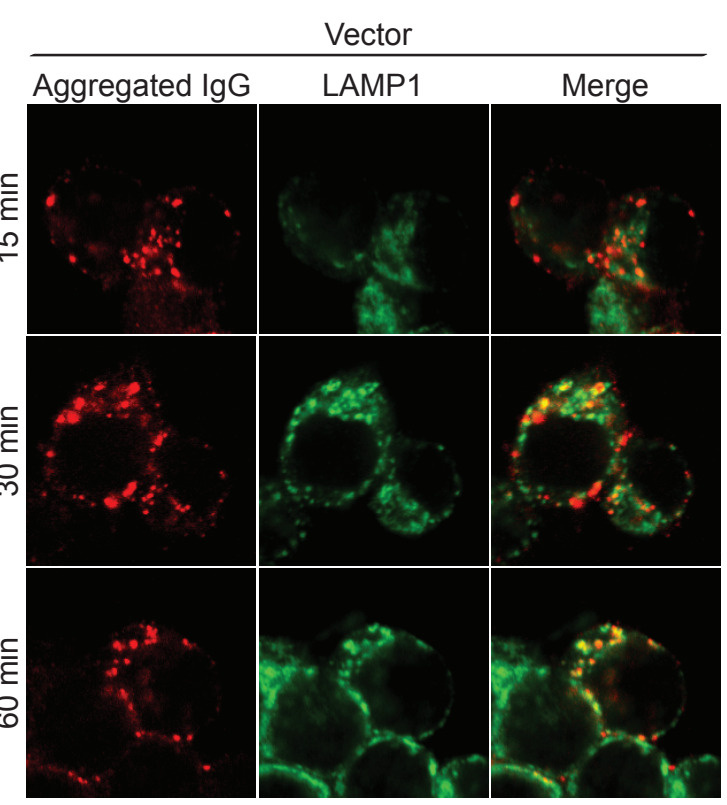

B

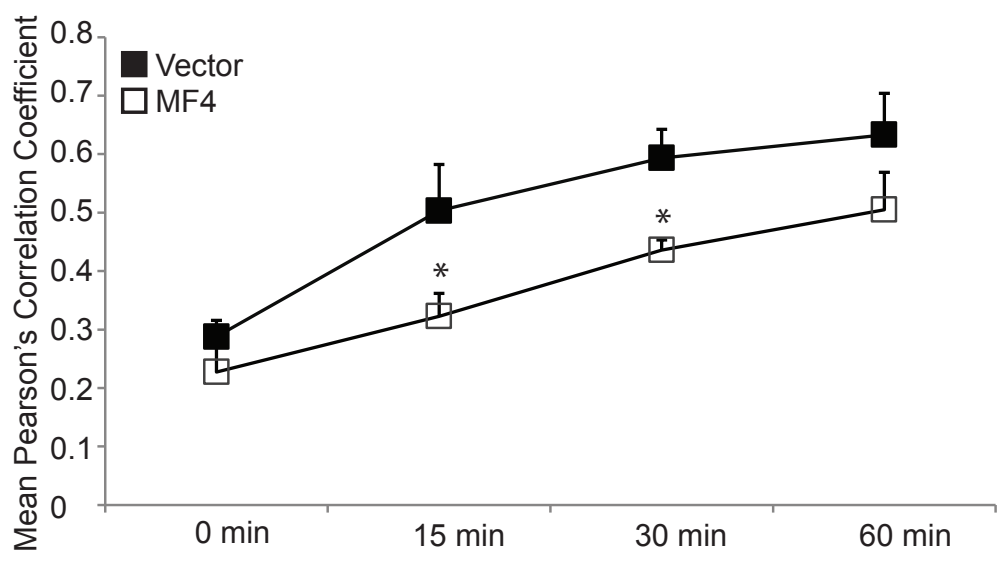

Figure 2.6: PIKfyve is required for efficient trafficking of immune complexes to lysosomes.

A. Cells were either treated with vector (control), or incubated with $100 \mathrm{nM} \mathrm{MF4}$ for $2 \mathrm{~h}$. Cells were then allowed to endocytose aggregated IgG for $15 \mathrm{~min}$, followed by washing to remove excess aggregated IgG and then chased for $0,15,30$ and 60 min at $37^{\circ} \mathrm{C}$. At 
the end of each chase, cells were fixed and processed to stain for LAMP1 and aggregated IgG. Images were acquired by confocal microscopy. B. Images were analysed with Image $J$ to acquire Pearson's Correlation Coefficient as an indicator of aggregated IgG co-localization with LAMP1. On average, ten cells for each condition across at least three experiments were quantified. Shown is the average Pearson's correlation normalized to 1 for control, 0 min chase. Error bars represent standard deviation. Asterisks indicate time points that were statistically different $(p<0.01)$ between control and MF4-treated cells using one-way ANOVA, followed by Tukey's post-hoc test. Scale bar is $10 \mu \mathrm{m}$.

\subsubsection{Phagosomal and lysosomal acidification does not require PIKfyve}

Since phagosomes in PIKfyve-quelled cells had reduced LAMP1 staining, we next examined if this affected two essential properties of phagolysosomes - acidification and proteolytic activity. We first inspected the ability of phagosomes to become acidic by staining cells with Lysotracker Green, a dye that accumulates in acidic compartments. After allowing phagosomes to form and mature for $1 \mathrm{~h}$ in control and $2 \mathrm{~h}$ MF4-treated cells, we observed that Lysotracker Green stained both sets of phagosomes equally well, suggesting that phagosomes acidified (Fig. 2.7A). Indeed, we confirmed that Lysotracker Green accumulates in lysosomes in control and MF4-treated macrophages as well (Fig.

2.7B). In these experiments, lysosomes were identified by pre-loading with Alexa647conjugated dextran, followed by incubation with MF4 and lastly with Lysotracker Green. In both control and MF4-treated cells, the co-localization of Alexa647-conjugated dextran and Lysotracker Green exhibited similar Pearson's correlation value of $0.43 \pm 0.06$ and $0.52 \pm 0.07$ (Fig. $2.7 C$ ). The role of PIKfyve in lysosomal acidification remains controversial. There are studies indicating that PIKfyve is necessary for efficient acidification of lysosomes in C. elegans, mammalian cells, Salmonella-containing 
vacuoles, as well as the yeast vacuole $(28,29,52,54)$. However, upon more careful inspection, Lysotracker is able to accumulate in various compartments and decorate the limiting membrane of swollen vacuoles $(52,54)$. Regardless, our observations in macrophages may very well represent a physiological difference between macrophages and other model systems or method employed.

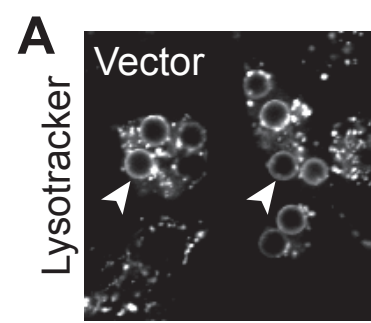

B

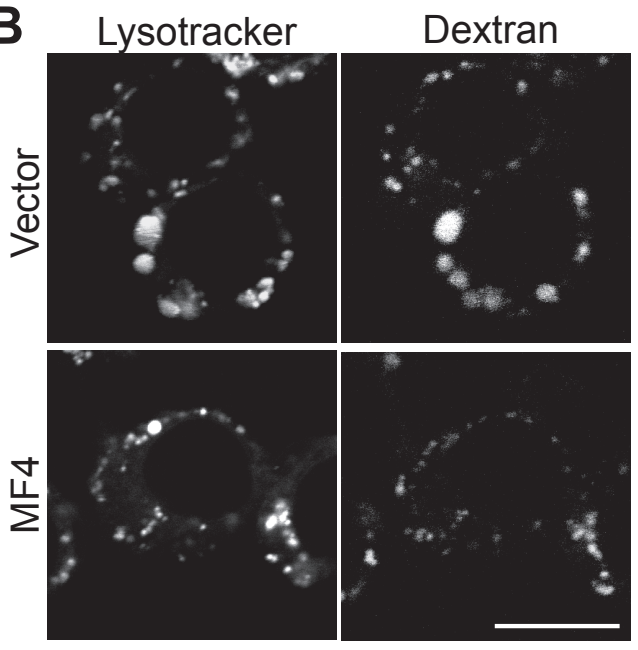

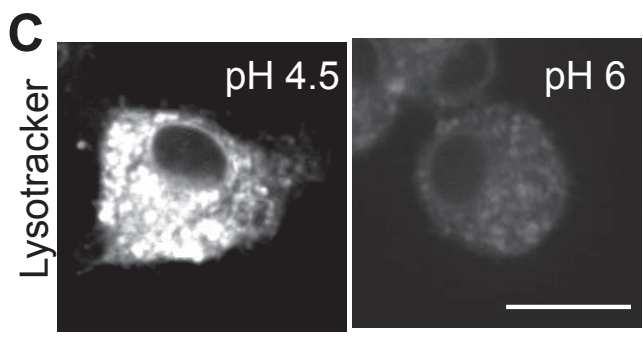

D

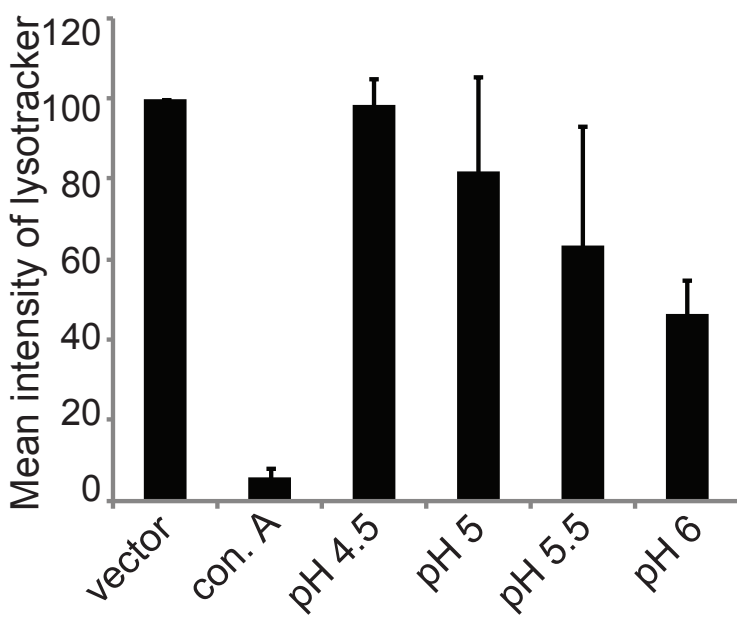

Figure 2.7: Phagosomes and lysosomes acidify independently of PIKfyve.

A. To test whether PIKfyve is required for phagosome acidification, RAW macrophages were treated with vector (control) or $2 \mathrm{~h} 100 \mathrm{nM} \mathrm{MF4}$ and then allowed to phagocytose IgG-opsonized beads for $15 \mathrm{~min}$ and chased for $60 \mathrm{~min}$. After the chase, cells were stained with Lysotracker Green for 5 min and observed by live-cell microscopy. Arrows point to phagosomes labeled with Lysotracker Green. B. To test whether lysosomes acidified in the absence of PIKfyve, lysosomes were pre-labelled with a 15 min pinocytic uptake with Alexa647-dextran followed by a 60 min chase. Cells were then labeled with Lysotracker Green as before and observed by live-cell microscopy. Shown is the Pearson's correlation coefficient for dextran and Lysotracker Green co-localization in control and MF4-treated cells. Scale bar is $10 \mu \mathrm{m}$. 
2.4.8 PIKfyve is important for efficient phagosomal and lysosomal proteolytic activity

Phagolysosome biogenesis confers the phagosome with hydrolytic activity to digest the engulfed particle. To examine the proteolytic proficiency of phagosomes in PIKfyveinhibited cells, we used sheep red blood cells (RBCs) opsonized with rabbit anti-sheep RBCs and cross-linked to DQ-BSA. When intact, DQ-BSA fluorescence is quenched. As DQ-BSA is degraded in lysosomes, it becomes dequenched. Thus, one can measure the increase in DQ-BSA fluorescence to quantify proteolytic activity. DQ-BSA-labelled phagosomes were allowed to form for $15 \mathrm{~min}$ and matured for $30 \mathrm{~min}$ before fixing, imaging and analysing for the presence or absence of DQ-BSA fluorescence. After scoring over 300 phagosomes per condition per experiment, we noted that MF4-treated cells had significantly fewer phagosomes with DQ-BSA fluorescence relative to control phagosomes (Fig. 2.8A,B). Thus, our data suggest that PIKfyve activity is necessary to endow phagosomes with a degradative milieu conducive to decomposing engulfed particles.

To complement this functional assay, we also quantified the level of cathepsin Dassociated with phagosomes by flow cytometry. Phagosomes from control and from MF4- or YM201636-treated cells were isolated, processed and stained for anti-cathepsin D antibodies, a canonical lysosomal protease (75). As shown in Figure $8 \mathrm{E}$, treatment with MF4 or YM201636 significantly reduced the amount of cathepsin D associated with isolated phagosomes relative to phagosomes from control cells. This result is consistent with reduced phagosome-associated proteolytic activity measured with the DQ-BSA assay and with reduced phagosome-associated LAMP1 signal in PIKfyve-inhibited cells. 
The proteolytic defect in phagosomes from PIKfyve-blocked cells might be caused by impaired lysosome fusion. Alternatively, or in addition, lysosomes themselves may have reduced proteolytic activity. To test this possibility, we examined if lysosomes in PIKfyve-abated cells displayed impeded proteolytic activity. To do this, we allowed cells to co-endocytose DQ-BSA and TRITC-dextran, followed by a chase of 0 , 30 and 60 min to allow transport of the fluid-phase to lysosomes. By microscopy, it appeared that MF4-treated cells had less DQ-BSA (Fig. 2.8C). However, we also noted that MF4-treated cells appeared to have less dextran. Using flow cytometry, we confirmed that MF4-treated cells did accumulate less dextran compared to control cells (Fig. S2.2). Interestingly, others have also observed a reduction if fluid-phase uptake in HEK cells expressing PIKfyve dominant-negative (76).

Thus, to compensate for differences in fluid-phase uptake or retention between control and MF4-treated cells, we normalized DQ-BSA fluorescence over Alexa647dextran fluorescence measured by flow cytometry. As expected, there was very weak DQ-BSA signal in both control and MF4-treated cells 0 min post-pinocytosis (Fig. 2.8C,D). By contrast, there was a significant and progressive increase in DQ-BSA fluorescence after 30 and 60 min post-pinocytosis in control cells (Fig. 2.8C,D). Importantly, the rate of DQ-BSA degradation in MF4-treated cells was slower relative to control cells; at 30 min the fluorescence ratio of DQ-BSA/Alexa647-dextran in MF4-treated cells was significantly reduced relative to control cells $(1.44 \pm 0.64$ vs $0.84 \pm 0.27$; Fig. $2.8 \mathrm{D})$. After $60 \mathrm{~min}$, there was no statistical difference between control and MF4-treated cells (Fig. 2.8D). 
Comparing phagosome and lysosomal proteolytic activity, we can conclude that PIKfyve plays an important role in maintaining efficient proteolytic capacity of lysosomes and phagosomes in macrophages. This may be because PIKfyve appears to play a role in trafficking and recycling of mannose-6-phosphate receptors that shuttle between late endosome and the Golgi to deliver newly synthesized lysosomal proteases to lysosomes $(33,34,77)$. Reduced proteolytic activity of phagosomes may have significant implications towards removal of pathogens from cells and may also help explain why PIKfyve-quelled cells tend to accumulate or fail to mature autophagosomes $(34,35,78)$. 
A

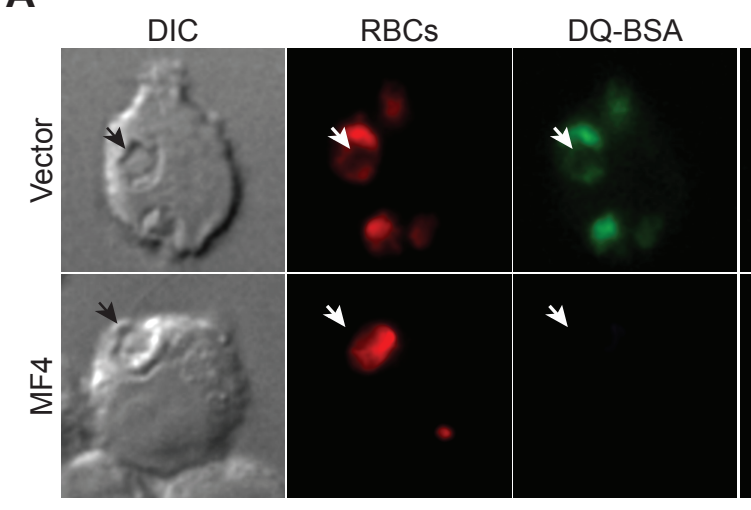

C

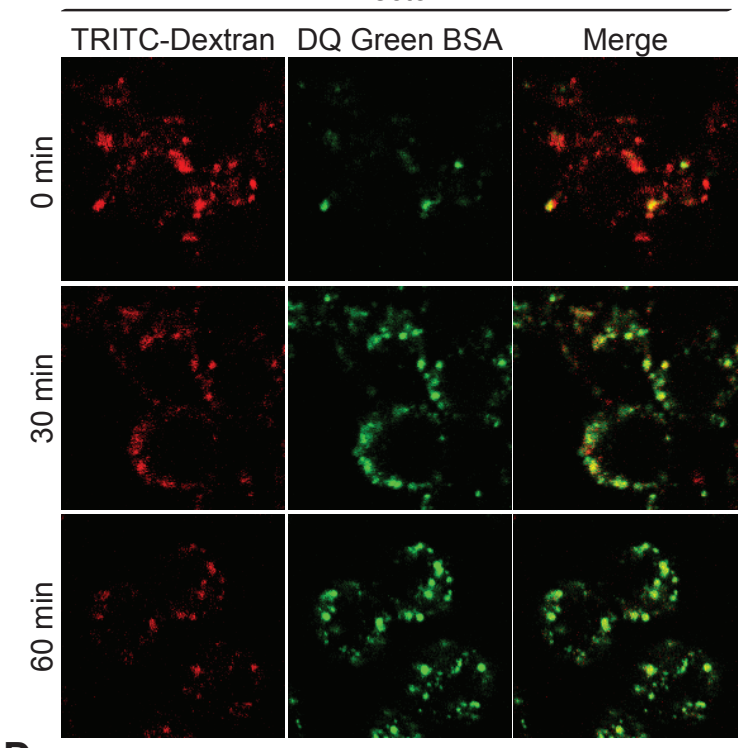

D

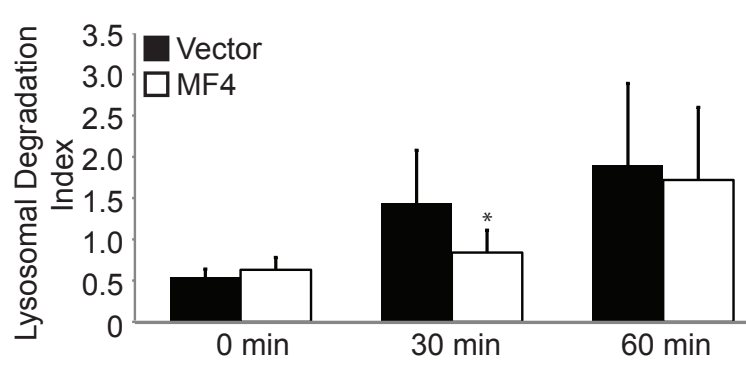

Merge
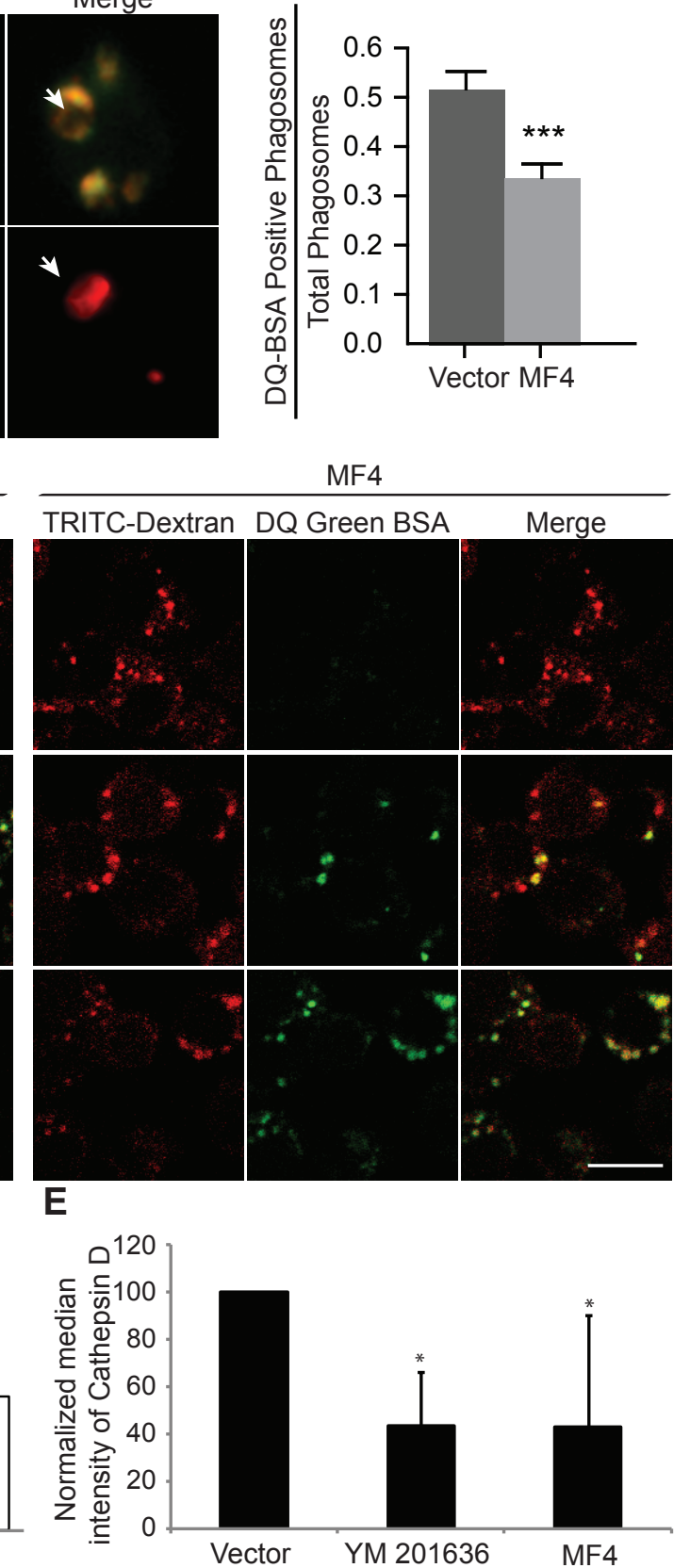

G

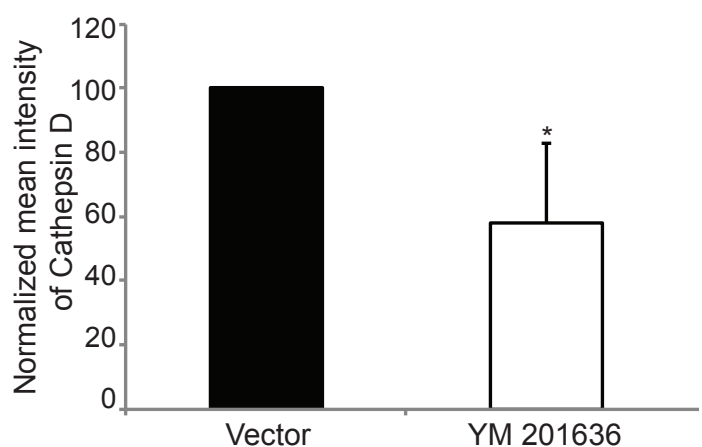


Figure 2.8: PIKfyve inhibition impairs proteolytic activity of phagosomes and lysosomes.

A. Control and cells treated with $200 \mathrm{nM}$ MF4 for $2 \mathrm{~h}$ were allowed to phagocytose RBCs cross-linked with DQ-BSA for $15 \mathrm{~min}$, followed by a $30 \mathrm{~min}$ chase. RBC-containing phagosomes are shown in red, while DQ-BSA was observed in the green channel. The arrows indicate the position of the same phagosome in each panel. Corresponding DIC images of cells are also shown. B. Percent of DQ-BSA positive phagosomes in control and MF4-treated cells. Data shown are means \pm SEM from three independent experiments. At least 300 phagosomes were analyzed in each case. A statistically significant difference between control and MF4 treated cells was observed at 30 min $(p=0.0001)$ using Student's t-test. Scale bars, $5 \mu \mathrm{m}$. C. Control and cells treated with $100 \mathrm{nM}$ MF4 for $2 \mathrm{~h}$ were allowed to co-endocytose TRITC-dextran and DQ-BSA for 15 min, followed by 0,30 or $60 \mathrm{~min}$ chase. Cells were then observed by confocal microscopy. Scale bar is $10 \mu \mathrm{m}$. D. Fluorescence ratio of DQ-BSA over fluorescent dextran in control and MF4-treated cells. After pinocytosis and the indicated chase period, cells were fixed and analysed by flow cytometry. After background correction, dextran-positive cells were analysed for mean fluorescence for dextran and DQ-BSA. Shown is the average ratio of DQ-BSA to dextran and \pm standard deviation. A statistically significant difference between control and MF4-treated cells existed at 30 min $(p<0.05)$ based on a one-way ANOVA test and Tukey's post-hoc test. E. Median cathepsin D-associated fluorescence intensity of isolated phagosomes from control cells and cells treated with YM201636 or MF4. Fluorescence intensities are normalized to control phagosomes and background corrected. Using one-way ANOVA and Tukey's ttest, there was significant difference in cathepsin $D$ staining between control and drugtreated cells $(p<0.01)$.

Overall, the work presented here examines the effect of inhibiting PIKfyve on various parameters of the macrophage endolysosomal and phagosomal system. We show that PIKfyve is necessary for phagosome maturation, trafficking to lysosomes of immune complexes and maintaining the degradative milieu of lysosomes and phagosomes. However, while we speculate that these functions rely on PIKfyvedependent $\mathrm{PI}(3,5) \mathrm{P}_{2}$ synthesis, we cannot currently exclude that some of our observations may be due to concurrent loss of PI(5)P. There are several lines of 
evidence that PIKfyve may directly synthesize $\mathrm{PI}(5) \mathrm{P}$, or indirectly, through the dephosphorylation of $\mathrm{PI}(3,5) \mathrm{P}_{2}$ by a myotubularin $(59,60,79)$.

\subsection{Materials and Methods}

\subsubsection{Nucleic acids and antibodies}

The plasmid encoding 2XFYVE-GFP was previously characterized (49). Plasmids were extracted and purified using E.Z.N.A. ${ }^{\circledR}$ Plasmid Midiprep Kit (Omega Bio-Tek) as per manufacturer's instructions. For PIKfyve gene silencing by siRNA, we used SMARTpool containing four oligonucleotides and the corresponding non-targeting oligonucleotide sets (Fisher Scientific, Canada, ON). The primary antibodies used in this study were rat anti-mouse LAMP1 monoclonal antibodies (clone 1D4B used at 1:200, Developmental Hybridoma Bank, IA), rabbit anti-mouse EEA1 (used at 1:400, Cell Signaling, MA) and rabbit anti-cathepsin D antibodies (clone EPR3057Y, 1:200 Genetex, CA). Fluorescent secondary antibodies used were at 1:200 to 1:1000 (Life Technologies, CA).

\subsubsection{Cell culture, transfection and electroporation}

RAW264.7 macrophages were obtained from ATCC and cultured in Dulbecco's modified Eagle's medium (DMEM) supplemented with high glucose, L-glutamine, sodium pyruvate, and $10 \%$ heat-inactivated fetal bovine serum (Gibco) at $37{ }^{\circ} \mathrm{C}$ in $5 \% \mathrm{CO}_{2}$. For experiments in ambient $\mathrm{CO}_{2}$, RAW cells were incubated in HEPES-buffered RPMI.

Prior to transfection, cells were seeded onto glass coverslips to reach $50 \%$ confluency. Plasmids were transfected into cultured cells using Fugene HD (Promega) 
transfection reagent as per manufacturer's instructions. For gene silencing, cells were seeded onto T25 flasks to reach $90 \%$ confluency. Electroporation of cells with SMARTpool oligonucleotides was done by an Amaxa system by two rounds of electroporation over $24 \mathrm{~h}$, followed by incubation in DMEM plus 10\% FBS for $48 \mathrm{~h}$ at $37^{\circ} \mathrm{C}$ in $5 \% \mathrm{CO}_{2}$.

\subsubsection{Pharmacological treatments}

To inhibit PIKfyve, cells were pre-incubated for the indicated period of time and concentration of MF4 (a kind gift from Dr. Kevan Shokat, UCSF), YM201636 (Chemdea, NJ or Cedarlane, ON) or apilimod (Toronto Research Chemicals, Inc, ON). To inhibit PtdIns 3-kinases, we typically used $100 \mu \mathrm{M}$ LY294002 (Promega) for 30 min. Drugs were maintained through the entire experiment including pulse and chase periods when performing phagocytosis, phagosome maturation or endosomal trafficking.

\subsubsection{Phagocytosis and phagosome maturation assays}

Latex polymer beads with a mean diameter of $3.87 \mu \mathrm{m}$ (Bangs Laboratories, IN) were opsonized with $10 \mathrm{mg} / \mathrm{ml}$ human IgG in PBS at room temperature for $1-2 \mathrm{~h}$ rotating. Opsonized beads were washed 3 times in PBS with centrifugation at $2000 \mathrm{rpm}$ for $1 \mathrm{~min}$ between each wash. To synchronize phagocytosis, IgG-opsonized beads were resuspended in ice-cold HPMI and added to cells for 20 min on ice. Subsequently, cells were washed twice with PBS to remove unbound beads and incubated at $37^{\circ} \mathrm{C}$ for 15 min for phagocytosis. For phagocytic index, cells were then fixed with 4\% PFA for 20 min, quenched with $100 \mathrm{mM}$ glycine for $20 \mathrm{~min}$, and stained with fluorescently labelled 
goat anti-human antibodies at 1:1000 dilution for $20 \mathrm{~min}$ to discriminate internalized beads from externally bound beads. The number of phagosomes per 100 cells was scored to determine the phagocytic index. Alternatively, after phagocytosis, cells were incubated at $37{ }^{\circ} \mathrm{C}$ for a chase period to allow for phagosome maturation before processing for the next step. For live-cell imaging of phagocytosis, IgG-coated beads were added to cells and imaged to track nascent phagosomes (see below for imaging details).

\subsubsection{Immunofluorescence}

After pulse-chase experiments that required immuno-staining, cells were fixed with $4 \%$ PFA for $20 \mathrm{~min}$ and quenched with $100 \mathrm{mM}$ glycine for $20 \mathrm{~min}$. When necessary, external beads were then stained with anti-human IgG and then cells were permeabilized with either $0.5 \%$ Triton $X-100$ for 10 min to stain for EEA1, cathepsin D or aggregated IgG, or treated with ice-cold methanol for 5 min to stain or co-stain with anti-LAMP1 antibodies. Cells were washed 3 times with $0.5 \%$ bovine serum albumin (BSA)/PBS prior to and after incubation with primary antibody, followed by secondary antibody staining, each at room temperature and each incubated for $1 \mathrm{~h}$. Finally, cells were washed and incubated with $0.5 \%$ BSA/PBS every 5 min for $30 \mathrm{~min}$. Cells were then visualized by confocal microscopy.

\subsubsection{Phagosome isolation}

RAW macrophages were grown to $80 \%$ confluency in T-25 culture flasks. Cells were then pre-treated with each drug for $1 \mathrm{~h}$ and IgG-opsonized beads were then added and 
incubated for 90 min at $37^{\circ} \mathrm{C}$ and $5 \% \mathrm{CO}_{2}$. Cells were washed $3 \mathrm{X}$ with ice-cold PBS to remove unbound beads and scraped in $15 \mathrm{ml}$ of homogenization buffer ( $20 \mathrm{mM}$ Tris $\mathrm{pH}$ 7.4, $2.5 \mu \mathrm{L} / \mathrm{ml}$ protease inhibitor cocktail (Sigma), $1 \mathrm{mM} \mathrm{AEBSF,} 1 \mathrm{mM} \mathrm{MgCl}, 1 \mathrm{mM}$ $\mathrm{CaCl}_{2}, 1 \mu \mathrm{g} / \mathrm{ml}$ RNase and $1 \mu \mathrm{g} / \mathrm{ml}$ DNase). After centrifuging, the cell pellet was resuspended in $2 \mathrm{~mL}$ of homogenization buffer and passed through a syringe with 22gauge needle (20-25 times) to lyse the cells. A pellet was then obtained after membrane disruption, and re-suspended in $200 \mu \mathrm{L}$ of PBS and overlaid onto a sucrose gradient. Samples were then centrifuged at 50,000g for $10 \mathrm{~min}$ at $4^{0} \mathrm{C}$, the layer of beads were withdrawn from the sucrose gradient using a syringe with 22-gauge needle and transferred into a fresh microcentrifuge tube. Phagosomes were then washed with cold PBS and fixed with 4\% PFA for 20 min, followed by quenching with $100 \mathrm{mM}$ of glycine. After centrifugation to collected fixed phagosomes, phagosomes were permeablized with iced-cold methanol for 3-5 min and washed $3 \mathrm{X}$ with $0.5 \%$ BSA to stain with antiLAMP1 antibodies. For cathepsin D staining, fixed phagosomes were permeablized with 0.5\% Triton $\mathrm{X}$ for $10 \mathrm{~min}$. Phagosomes were then processed as described in "Immunofluorescence" and analysed by flow cytometry.

\subsubsection{Endosomal trafficking of aggregated IgG}

Aggregated IgG was made by heating $10 \mathrm{mg} / \mathrm{ml}$ human IgG (Sigma-Aldrich) at $62{ }^{\circ} \mathrm{C}$ for $20 \mathrm{~min}$, followed by centrifugation at $16,000 \mathrm{~g}$ for $10 \mathrm{~min}$ at room temperature to sediment insoluble complexes. Cells were incubated with $1 \mathrm{mg} / \mathrm{mL}$ soluble aggregated IgG at $37{ }^{\circ} \mathrm{C}$ for $15 \mathrm{~min}$. Subsequently, cells were washed briefly with PBS to remove excess aggregated IgG and followed by a chase period at $37^{\circ} \mathrm{C}$. After the chase period, 
cells were fixed and stained for aggregated IgG and LAMP1 as described below under "immunofluorescence".

\subsubsection{Acidification of lysosomes and phagosomes}

Lysosomes were labeled with dextran by preloading cells with $0.1 \mathrm{mg} / \mathrm{ml}$ FITCconjugated dextran at $37{ }^{\circ} \mathrm{C}$ for $1 \mathrm{~h}$. Cells were then washed briefly with PBS to remove excess dextran and incubated in dye-free media at $37{ }^{\circ} \mathrm{C}$ for a subsequent chase period

of $2 \mathrm{~h}$. To label lysosomes with Lysotracker Green (Life Technologies, CA), cells were incubated with $1 \mu \mathrm{M}$ Lysotracker at $37^{\circ} \mathrm{C}$ for 5 min just prior to live-cell imaging. Cells containing phagosomes were similarly stained with Lysotracker Red.

\subsubsection{Preparation of DQ-BSA cross-linked RBCs}

DQ-BSA cross-linked RBCs were prepared as described previously (80). Briefly, RBCs were opsonized with $0.1 \mathrm{mg} / \mathrm{ml}$ of anti-sheep RBC antibody for $1 \mathrm{~h}$, followed by $1 \mathrm{~h}$ labeling with secondary antibody to visualize RBCs. Cells were then re-suspended in $1 \mathrm{X}$ PBS with $25 \mathrm{mg} / \mathrm{ml}$ carbodiimide cross-linker and agitated for $15 \mathrm{~min}$. Excess carbodiimide was removed by three washes in $1 \mathrm{ml}$ of $0.1 \mathrm{M}$ sodium borate, $\mathrm{pH} 8.0$ (coupling buffer). Cells were re-suspended in $500 \mu \mathrm{l}$ of coupling buffer containing $1.0 \mathrm{mg}$ DQ-ovalbumin and $0.1 \mathrm{mg} / \mathrm{ml}$ of anti-sheep RBC antinody for 12 hours. DQ-BSA coated RBCs were washed three times with $250 \mathrm{mM}$ glycine in PBS, pH 7.2 (quench buffer) by vortexing and centrifugation at 3000xg for 5 min, followed by three washes with PBS before use in a phagocytic assay. 


\subsubsection{DQ-BSA proteolysis assay by endocytosis}

To determine the degradative capacity of lysosomes, cells were allowed to coendocytose $10 \mu \mathrm{g} / \mathrm{ml}$ DQ Green BSA (Life Technologies, CA) and $0.1 \mathrm{mg} / \mathrm{ml}$ tetramethylrhodamine isothiocyanate (TRITC)-conjugated dextran or $2 \mu \mathrm{M}$ Alexa647conjugated dextran (Life Technologies,) at $37^{\circ} \mathrm{C}$ for $15 \mathrm{~min}$. Subsequently, cells were incubated at $37^{\circ} \mathrm{C}$ in the absence of the labels for 0,30 , or $60 \mathrm{~min}$. Cells were then fixed with 4\% PFA for 20 min and quenched with $100 \mathrm{mM}$ glycine for $20 \mathrm{~min}$, followed by microscopy or flow cytometry.

\subsubsection{Microscopy and image processing}

For confocal imaging, cells were visualized on a Zeiss LSM 510 META laser-scanning microscope. This instrument is equipped with an Argon laser (458/477/488/514 nm), a HeNe1 laser $(543 \mathrm{~nm})$, and a HeNe2 laser (633 nm). Plan-Apochromat 100x/1.40 oil objective was used to collect images.

For live-cell imaging, cells on coverslips were placed on a Leiden chamber and incubated with Hepes-buffered RPMI medium at 37 ㅇ. Cells were imaged by differential interference contrast (DIC) to score for vacuolation. To follow 2FYVE-GFP dynamics, time series composed of non-saturated 8-bit grayscale frames were acquired at two frames per min. Images where then analysed by ImageJ by identifying regions of interest on the phagosomal membrane, cytosol and extracellular space (for background subtraction). Fluorescence intensity values were then extracted, followed by background substraction and the ratio of phagosome to cytosol signal was calculated and plotted as an indicator of 2FYVE-GFP intensity on phagosomes. 
DQ-BSA-phagosome imaging was done by epifluorescence microscopy using $63 \mathrm{X}$ oil immersion lens (Leica DMI6000B stand with a Hamamatsu EM-1 K, EMCCD camera). Image acquisition was performed using Volocity software (Improvision).

Images were processed with Adobe Photoshop (v. 7.0.1, Adobe Systems Inc.) and analyzed using ImageJ (v. 1.47 bundled with 64-bit Java). All image processing was done without altering relative values within each image and when comparing images. Pseudo-colour processing was done with ImageJ by enabling "Fire" in Image/Look up Tables such that white-yellow corresponded to grayscale intensities of $255-180$, orangered corresponded to grayscale intensities of $180-80$ and blue-purple to grayscale intensities of 80-1. Co-localization analysis was done using ImageJ equipped with the plugins, JACoP (Just Another Colocalization Plugin) or Colocalization Indices.

\subsubsection{Flow cytometry analysis}

Endocytosis and DQ-BSA fluorescence was quantified using a BD FACSCalibur flow cytometer. Samples of suspended cells were run through the flow cytometer until 10,000 events were collected per condition. Data obtained using flow cytometry was analyzed using either Flowjo or Flowing Software. After gating for background fluorescence, the extent of lysosomal proteolysis was quantified in cells positive for Alexa647-dextran and then calculating the average ratio of the mean fluorescence of DQ-Green-BSA over the mean fluorescence of Alexa647-dextran.

To quantify anti-LAMP1 and anti-cathepsin D phagosome staining by flow cytometry, we counted 10,000 events for each sample and defined the phagosome population. We then used phagosomes incubated with secondary-only (no primary) to 
gate for background staining. We counted the number of phagosomes that were in the $95^{\text {th }}$ percentile of background fluorescence and calculated the median fluorescence of these phagosomes for each sample.

\subsubsection{Statistical Analyses}

All data was subject to statistical analysis using either ANOVA, unpaired student t-test or paired Student's t-test as appropriate. Experiments were repeated a minimum of three times. Specific sample size is indicated in each figure legend, along with the determined mean, standard deviation and p-values.

\subsection{Acknowledgements}

The research described here was funded by a Discovery Grant from the Natural Sciences and Engineering Research Agency of Canada, by a Dean's Research Fund and a Health Sciences Research Grant from Ryerson University to R.J.B. MF4 was a kind gift from Dr. Kevan Shokat at the University of California, San Francisco. We thank Mr. Ryan Shilliday for help in scoring vacuole size and Mr. Christopher Spring from St. Michael's Hospital Flow Cytometry Facility for advice on flow cytometry. The 1D4B anti-LAMP1 monoclonal antibody was obtained from the Developmental Studies Hybridoma Bank developed under the auspices of the NICHD and maintained by The University of lowa, Department of Biology, lowa City, IA 52242. The authors have no conflict of interest to declare. 


\subsection{References}

1. Behnia R, Munro S. Organelle identity and the signposts for membrane traffic. Nature 2005;438(7068):597-604.

2. Botelho RJ. Changing phosphoinositides "on the fly": how trafficking vesicles avoid an identity crisis. Bioessays 2009.

3. Di Paolo G, De Camilli P. Phosphoinositides in cell regulation and membrane dynamics. Nature 2006;443(7112):651-657.

4. Burd CG, Emr SD. Phosphatidylinositol(3)-phosphate signaling mediated by specific binding to RING FYVE domains. Mol Cell 1998;2(1):157-162.

5. Stauffer TP, Ahn S, Meyer T. Receptor-induced transient reduction in plasma membrane Ptdlns(4,5)P2 concentration monitored in living cells. Curr Biol $1998 ; 8(6): 343-346$.

6. Varnai P, Balla T. Visualization of phosphoinositides that bind pleckstrin homology domains: calcium- and agonist-induced dynamic changes and relationship to myo-[3H]inositol-labeled phosphoinositide pools. J Cell Biol 1998;143(2):501-510.

7. Varnai P, Rother KI, Balla T. Phosphatidylinositol 3-kinase-dependent membrane association of the Bruton's tyrosine kinase pleckstrin homology domain visualized in single living cells. J Biol Chem 1999;274(16):10983-10989.

8. Nicot AS, Laporte J. Endosomal phosphoinositides and human diseases. Traffic 2008;9(8):1240-1249. 
9. Schmid S, Fuchs R, Kielian M, Helenius A, Mellman I. Acidification of endosome subpopulations in wild-type Chinese hamster ovary cells and temperature-sensitive acidification-defective mutants. J Cell Biol 1989;108(4):1291-1300.

10. Schmid SL, Fuchs R, Male P, Mellman I. Two distinct subpopulations of endosomes involved in membrane recycling and transport to lysosomes. Cell 1988;52(1):73-83.

11. Huotari J, Helenius A. Endosome maturation. Embo J 2011;30(17):3481-3500.

12. Dunn KW, Maxfield FR. Delivery of ligands from sorting endosomes to late endosomes occurs by maturation of sorting endosomes. J Cell Biol 1992;117(2):301-310.

13. Dunn KW, McGraw TE, Maxfield FR. Iterative fractionation of recycling receptors from lysosomally destined ligands in an early sorting endosome. J Cell Biol 1989;109(6 Pt 2):3303-3314.

14. Bishop N, Horman A, Woodman P. Mammalian class E vps proteins recognize ubiquitin and act in the removal of endosomal protein-ubiquitin conjugates. J Cell Biol 2002;157(1):91-101.

15. Katzmann DJ, Babst $M$, Emr SD. Ubiquitin-dependent sorting into the multivesicular body pathway requires the function of a conserved endosomal protein sorting complex, ESCRT-I. Cell 2001;106(2):145-155.

16. Katzmann DJ, Stefan CJ, Babst M, Emr SD. Vps27 recruits ESCRT machinery to endosomes during MVB sorting. J Cell Biol 2003;162(3):413-423. 
17. Gillooly DJ, Morrow IC, Lindsay M, Gould R, Bryant NJ, Gaullier JM, Parton RG, Stenmark H. Localization of phosphatidylinositol 3-phosphate in yeast and mammalian cells. Embo J 2000;19(17):4577-4588.

18. Gaullier JM, Simonsen A, D'Arrigo A, Bremnes B, Stenmark H, Aasland R. FYVE fingers bind Ptdlns(3)P. Nature 1998;394(6692):432-433.

19. Birkeland HC, Stenmark H. Protein targeting to endosomes and phagosomes via FYVE and PX domains. Curr Top Microbiol Immunol 2004;282:89-115.

20. Cheever ML, Sato TK, de Beer T, Kutateladze TG, Emr SD, Overduin M. Phox domain interaction with PtdIns(3)P targets the Vam7 t-SNARE to vacuole membranes. Nat Cell Biol 2001;3(7):613-618.

21. Xu Y, Hortsman H, Seet L, Wong SH, Hong W. SNX3 regulates endosomal function through its PX-domain-mediated interaction with Ptdlns(3)P. Nat Cell Biol $2001 ; 3(7): 658-666$.

22. Raiborg C, Bremnes B, Mehlum A, Gillooly DJ, D'Arrigo A, Stang E, Stenmark H. FYVE and coiled-coil domains determine the specific localisation of Hrs to early endosomes. J Cell Sci 2001;114(Pt 12):2255-2263.

23. Simonsen A, Lippe R, Christoforidis S, Gaullier JM, Brech A, Callaghan J, Toh BH, Murphy C, Zerial M, Stenmark H. EEA1 links PI(3)K function to Rab5 regulation of endosome fusion. Nature 1998;394(6692):494-498.

24. Christoforidis S, McBride HM, Burgoyne RD, Zerial M. The Rab5 effector EEA1 is a core component of endosome docking. Nature 1999;397(6720):621-625. 
25. Bache KG, Brech A, Mehlum A, Stenmark H. Hrs regulates multivesicular body formation via ESCRT recruitment to endosomes. J Cell Biol 2003;162(3):435-442.

26. Shisheva A, Rusin B, Ikonomov OC, DeMarco C, Sbrissa D. Localization and insulin-regulated relocation of phosphoinositide 5-kinase PIKfyve in 3T3-L1 adipocytes. J Biol Chem 2001;276(15):11859-11869.

27. Ikonomov OC, Sbrissa D, Shisheva A. Mammalian cell morphology and endocytic membrane homeostasis require enzymatically active phosphoinositide 5-kinase PIKfyve. J Biol Chem 2001;276(28):26141-26147.

28. Gary JD, Wurmser AE, Bonangelino CJ, Weisman LS, Emr SD. Fab1p is essential for PtdIns(3)P 5-kinase activity and the maintenance of vacuolar size and membrane homeostasis. J Cell Biol 1998;143(1):65-79.

29. Nicot AS, Fares H, Payrastre B, Chisholm AD, Labouesse M, Laporte J. The phosphoinositide kinase PIKfyve/Fab1p regulates terminal lysosome maturation in Caenorhabditis elegans. Mol Biol Cell 2006;17(7):3062-3074.

30. Sbrissa D, Ikonomov OC, Shisheva A. PIKfyve, a mammalian ortholog of yeast Fab1p lipid kinase, synthesizes 5-phosphoinositides. Effect of insulin. J Biol Chem 1999;274(31):21589-21597.

31. Ikonomov OC, Sbrissa D, Mlak K, Kanzaki M, Pessin J, Shisheva A. Functional dissection of lipid and protein kinase signals of PIKfyve reveals the role of Ptdlns 3,5-P2 production for endomembrane integrity. J Biol Chem 2002;277(11):9206-9211.

32. Ikonomov OC, Sbrissa D, Mlak K, Deeb R, Fligger J, Soans A, Finley RL, Jr., Shisheva A. Active PIKfyve associates with and promotes the membrane attachment of 
the late endosome-to-trans-Golgi network transport factor Rab9 effector p40. J Biol Chem 2003;278(51):50863-50871.

33. Rutherford AC, Traer C, Wassmer T, Pattni K, Bujny MV, Carlton JG, Stenmark H, Cullen PJ. The mammalian phosphatidylinositol 3-phosphate 5-kinase (PIKfyve) regulates endosome-to-TGN retrograde transport. J Cell Sci 2006;119(Pt 19):3944-3957.

34. de Lartigue J, Polson H, Feldman M, Shokat K, Tooze SA, Urbe S, Clague MJ. PIKfyve regulation of endosome-linked pathways. Traffic 2009;10(7):883-893.

35. Martin S, Harper CB, May LM, Coulson EJ, Meunier FA, Osborne SL. Inhibition of PIKfyve by YM-201636 dysregulates autophagy and leads to apoptosis-independent neuronal cell death. PLoS One 2013;8(3):e60152.

36. Bridges D, Ma JT, Park S, Inoki K, Weisman LS, Saltiel AR. Phosphatidylinositol 3,5-bisphosphate plays a role in the activation and subcellular localization of mechanistic target of rapamycin 1. Mol Biol Cell 2012;23(15):2955-2962.

37. Dong XP, Shen D, Wang X, Dawson T, Li X, Zhang $Q$, Cheng $X$, Zhang $Y$, Weisman LS, Delling M, Xu H. PI(3,5)P(2) controls membrane trafficking by direct activation of mucolipin $\mathrm{Ca}(2+)$ release channels in the endolysosome. Nat Commun 2010;1:38.

38. Tsuruta F, Green EM, Rousset M, Dolmetsch RE. PIKfyve regulates CaV1.2 degradation and prevents excitotoxic cell death. J Cell Biol 2009;187(2):279-294.

39. Zhang X, Chow CY, Sahenk Z, Shy ME, Meisler MH, Li J. Mutation of FIG4 causes a rapidly progressive, asymmetric neuronal degeneration. Brain 2008;131(Pt 8):19902001. 
40. Zhang Y, McCartney AJ, Zolov SN, Ferguson CJ, Meisler MH, Sutton MA, Weisman LS. Modulation of synaptic function by VAC14, a protein that regulates the phosphoinositides $\mathrm{PI}(3,5) \mathrm{P}(2)$ and PI(5)P. Embo J 2012;31(16):3442-3456.

41. Zhang Y, Zolov SN, Chow CY, Slutsky SG, Richardson SC, Piper RC, Yang B, Nau JJ, Westrick RJ, Morrison SJ, Meisler MH, Weisman LS. Loss of Vac14, a regulator of the signaling lipid phosphatidylinositol 3,5-bisphosphate, results in neurodegeneration in mice. Proc Natl Acad Sci U S A 2007;104(44):17518-17523.

42. Armstrong JA, Hart PD. Phagosome-lysosome interactions in cultured macrophages infected with virulent tubercle bacilli. Reversal of the usual nonfusion pattern and observations on bacterial survival. J Exp Med 1975;142(1):1-16.

43. Vieira OV, Botelho RJ, Grinstein S. Phagosome maturation: aging gracefully. Biochem J 2002;366(Pt 3):689-704.

44. Leung KP, Allen RD. Phagosomal-phagolysosomal membrane dynamics of stimulated mouse peritoneal macrophages. Eur J Cell Biol 1982;29(1):1-12.

45. Pitt A, Mayorga LS, Schwartz AL, Stahl PD. Transport of phagosomal components to an endosomal compartment. J Biol Chem 1992;267(1):126-132.

46. Downey GP, Botelho RJ, Butler JR, Moltyaner $\mathrm{Y}$, Chien P, Schreiber AD, Grinstein S. Phagosomal maturation, acidification, and inhibition of bacterial growth in nonphagocytic cells transfected with FcgammaRIIA receptors. J Biol Chem 1999;274(40):28436-28444.

47. McNeil PL, Tanasugarn L, Meigs JB, Taylor DL. Acidification of phagosomes is initiated before lysosomal enzyme activity is detected. J Cell Biol 1983;97(3):692-702. 
48. Bouvier G, Benoliel AM, Foa C, Bongrand P. Relationship between phagosome acidification, phagosome-lysosome fusion, and mechanism of particle ingestion. J Leukoc Biol 1994;55(6):729-734.

49. Vieira OV, Botelho RJ, Rameh L, Brachmann SM, Matsuo T, Davidson HW, Schreiber A, Backer JM, Cantley LC, Grinstein S. Distinct roles of class I and class III phosphatidylinositol 3-kinases in phagosome formation and maturation. J Cell Biol 2001;155(1):19-25.

50. Fratti RA, Backer JM, Gruenberg J, Corvera S, Deretic V. Role of phosphatidylinositol 3-kinase and Rab5 effectors in phagosomal biogenesis and mycobacterial phagosome maturation arrest. J Cell Biol 2001;154(3):631-644.

51. Ellson CD, Anderson KE, Morgan G, Chilvers ER, Lipp P, Stephens LR, Hawkins PT. Phosphatidylinositol 3-phosphate is generated in phagosomal membranes. Curr Biol 2001;11(20):1631-1635.

52. Jefferies HB, Cooke FT, Jat P, Boucheron C, Koizumi T, Hayakawa M, Kaizawa H, Ohishi T, Workman P, Waterfield MD, Parker PJ. A selective PIKfyve inhibitor blocks Ptdlns $(3,5) P(2)$ production and disrupts endomembrane transport and retroviral budding. EMBO Rep 2008;9(2):164-170.

53. Hazeki K, Nigorikawa K, Takaba Y, Segawa T, Nukuda A, Masuda A, Ishikawa Y, Kubota K, Takasuga S, Hazeki O. Essential roles of PIKfyve and PTEN on phagosomal phosphatidylinositol 3-phosphate dynamics. FEBS Lett 2012;586(22):4010-4015. 
54. Kerr MC, Wang JT, Castro NA, Hamilton NA, Town L, Brown DL, Meunier FA, Brown NF, Stow JL, Teasdale RD. Inhibition of the Ptdlns(5) kinase PIKfyve disrupts intracellular replication of Salmonella. Embo J 2010;29(8):1331-1347.

55. Hazeki K, Uehara M, Nigorikawa K, Hazeki O. PIKfyve regulates the endosomal localization of CpG oligodeoxynucleotides to elicit TLR9-dependent cellular responses. PLoS One 2013;8(9):e73894.

56. Cai X, Xu Y, Cheung AK, Tomlinson RC, Alcazar-Roman A, Murphy L, Billich A, Zhang B, Feng Y, Klumpp M, Rondeau JM, Fazal AN, Wilson CJ, Myer V, Joberty G, et al. PIKfyve, a class III PI kinase, is the target of the small molecular IL-12/IL-23 inhibitor apilimod and a player in Toll-like receptor signaling. Chem Biol 2013;20(7):912-921.

57. Sbrissa D, Shisheva A. Acquisition of unprecedented phosphatidylinositol 3,5bisphosphate rise in hyperosmotically stressed 3T3-L1 adipocytes, mediated by ArPIKfyve-PIKfyve pathway. J Biol Chem 2005;280(9):7883-7889.

58. Ikonomov OC, Sbrissa D, Shisheva A. YM201636, an inhibitor of retroviral budding and PIKfyve-catalyzed $\mathrm{PI}(3,5) \mathrm{P} 2$ synthesis, halts glucose entry by insulin in adipocytes. Biochem Biophys Res Commun 2009;382(3):566-570.

59. Sbrissa D, Ikonomov OC, Filios C, Delvecchio K, Shisheva A. Functional dissociation between PIKfyve-synthesized Ptdlns5P and $\mathrm{PI}(3,5) \mathrm{P} 2$ by means of the PIKfyve inhibitor YM201636. Am J Physiol Cell Physiol 2012;303(4):C436-446.

60. Oppelt A, Lobert VH, Haglund K, Mackey AM, Rameh LE, Liestol K, Schink KO, Pedersen NM, Wenzel EM, Haugsten EM, Brech A, Rusten TE, Stenmark H, Wesche J. 
Production of phosphatidylinositol 5-phosphate via PIKfyve and MTMR3 regulates cell migration. EMBO Rep 2013;14(1):57-64.

61. Ikonomov OC, Sbrissa D, Shisheva A. Localized PtdIns 3,5-P2 synthesis to regulate early endosome dynamics and fusion. Am J Physiol Cell Physiol 2006;291(2):C393-404.

62. Sbrissa D, Ikonomov OC, Strakova J, Dondapati R, Mlak K, Deeb R, Silver R, Shisheva A. A mammalian ortholog of Saccharomyces cerevisiae Vac14 that associates with and up-regulates PIKfyve phosphoinositide 5-kinase activity. Mol Cell Biol 2004;24(23):10437-10447.

63. Michell RH. Inositol lipids: from an archaeal origin to phosphatidylinositol 3,5bisphosphate faults in human disease. Febs J 2013.

64. Bogatikov E, Munoz C, Pakladok T, Alesutan I, Shojaiefard M, Seebohm G, Foller M, Palmada M, Bohmer C, Broer S, Lang F. Up-regulation of amino acid transporter SLC6A19 activity and surface protein abundance by PKB/Akt and PIKfyve. Cell Physiol Biochem 2013;30(6):1538-1546.

65. Munoz C, Almilaji A, Setiawan I, Foller M, Lang F. Up-regulation of the inwardly rectifying $\mathrm{K}(+)$ channel Kir2.1 (KCNJ2) by protein kinase $\mathrm{B}(\mathrm{PKB} / \mathrm{Akt})$ and PIKfyve. J Membr Biol 2013;246(3):189-197.

66. Joshi T, Butchar JP, Tridandapani S. Fcgamma receptor signaling in phagocytes. Int J Hematol 2006;84(3):210-216.

67. Ho CY, Alghamdi TA, Botelho RJ. Phosphatidylinositol-3,5-bisphosphate: no longer the poor PIP2. Traffic 2012;13(1):1-8. 
68. Osborne SL, Wen PJ, Boucheron C, Nguyen HN, Hayakawa M, Kaizawa H, Parker PJ, Vitale N, Meunier FA. PIKfyve negatively regulates exocytosis in neurosecretory cells. J Biol Chem 2008;283(5):2804-2813.

69. Er EE, Mendoza MC, Mackey AM, Rameh LE, Blenis J. AKT facilitates EGFR trafficking and degradation by phosphorylating and activating PIKfyve. Sci Signal 2013;6(279):ra45.

70. Samie M, Wang X, Zhang X, Goschka A, Li X, Cheng X, Gregg E, Azar M, Zhuo Y, Garrity AG, Gao Q, Slaugenhaupt S, Pickel J, Zolov SN, Weisman LS, et al. A TRP channel in the lysosome regulates large particle phagocytosis via focal exocytosis. Dev Cell 2013;26(5):511-524.

71. Liu Y, Lai YC, Hill EV, Tyteca D, Carpentier S, Ingvaldsen A, Vertommen D, Lantier L, Foretz M, Dequiedt F, Courtoy PJ, Erneux C, Viollet B, Shepherd PR, Tavare JM, et al. Phosphatidylinositol 3-phosphate 5-kinase (PIKfyve) is an AMPK target participating in contraction-stimulated glucose uptake in skeletal muscle. Biochem J 2013.

72. Pryor PR, Mullock BM, Bright NA, Gray SR, Luzio JP. The role of intraorganellar $\mathrm{Ca}(2+)$ in late endosome-lysosome heterotypic fusion and in the reformation of Iysosomes from hybrid organelles. J Cell Biol 2000;149(5):1053-1062.

73. Han BK, Emr SD. Phosphoinositide $[\mathrm{PI}(3,5) \mathrm{P} 2]$ lipid-dependent regulation of the general transcriptional regulator Tup1. Genes Dev 2011;25(9):984-995.

74. Zhang CY, Booth JW. Divergent intracellular sorting of Fc\{gamma\}RIIA and Fc\{gamma\}RIIB2. J Biol Chem 2010;285(44):34250-34258. 
75. Oude Elferink RP, Van Doorn-Van Wakeren J, Strijland A, Reuser AJ, Tager JM. Biosynthesis and intracellular transport of alpha-glucosidase and cathepsin D in normal and mutant human fibroblasts. Eur J Biochem 1985;153(1):55-63.

76. Ikonomov OC, Sbrissa D, Foti M, Carpentier JL, Shisheva A. PIKfyve controls fluid phase endocytosis but not recycling/degradation of endocytosed receptors or sorting of procathepsin D by regulating multivesicular body morphogenesis. Mol Biol Cell 2003;14(11):4581-4591.

77. Bryant NJ, Piper RC, Weisman LS, Stevens TH. Retrograde traffic out of the yeast vacuole to the TGN occurs via the prevacuolar/endosomal compartment. J Cell Biol 1998;142(3):651-663.

78. Rusten TE, Vaccari T, Lindmo K, Rodahl LM, Nezis IP, Sem-Jacobsen C, Wendler F, Vincent JP, Brech A, Bilder D, Stenmark H. ESCRTs and Fab1 regulate distinct steps of autophagy. Curr Biol 2007;17(20):1817-1825.

79. Zolov SN, Bridges D, Zhang Y, Lee WW, Riehle E, Verma R, Lenk GM, ConversoBaran K, Weide T, Albin RL, Saltiel AR, Meisler MH, Russell MW, Weisman LS. In vivo, Pikfyve generates $\mathrm{PI}(3,5) \mathrm{P} 2$, which serves as both a signaling lipid and the major precursor for PI5P. Proc Natl Acad Sci U S A 2012;109(43):17472-17477.

80. VanderVen BC, Hermetter A, Huang A, Maxfield FR, Russell DG, Yates RM. Development of a novel, cell-based chemical screen to identify inhibitors of intraphagosomal lipolysis in macrophages. Cytometry A;77(8):751-760. 


\subsection{APPENDIX A}

\section{SUPPORTING INFORMATION FOR CHAPTER 2}



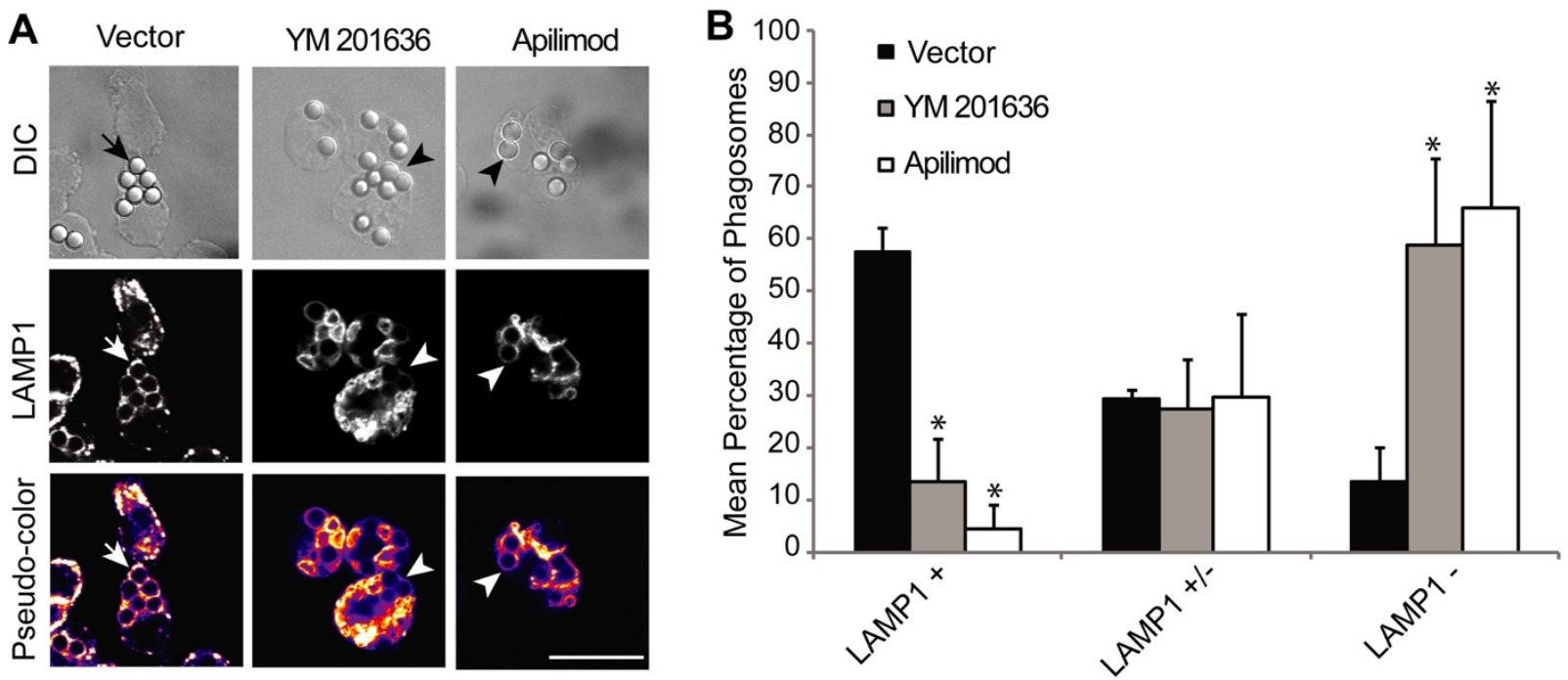

Figure S 2.1: YM201636 and apilimod treatments hinder phagosome maturation

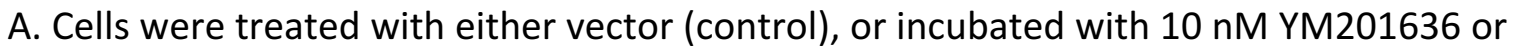
$25 \mathrm{nM}$ apilimod for $2 \mathrm{~h}$. Subsequently, phagocytosis and phagosome maturation were elicited, followed by fixation, staining for external beads and staining for LAMP1 as described in the legend of Figure 2.5. After microscopy, images were processed with pseudocolour to represent strong LAMP1 acquisition (LAMP1 ${ }^{+}$, white-yellow), intermediate LAMP1 decoration $\left(\mathrm{LAMP}^{+/-}\right.$, orange-red) and weak LAMP1 staining (LAMP1', blue-purple). Scale bar $=10 \mu \mathrm{m}$. B. At least 70 phagosomes per experiment were counted for each condition and for each of three independent experiments. Shown is the mean percent \pm standard deviation of phagosomes in each LAMP1intensity category. Using the one-way ANOVA, followed by Tukey's post-hoc test, we found that there was significant difference in the number of phagosomes stained strongly or weakly for LAMP1 between control and each PIKfyve inhibitor (ANOVA $p<0.0001$, Tukey's $p<0.01$ for samples indicated by the asterisk). 


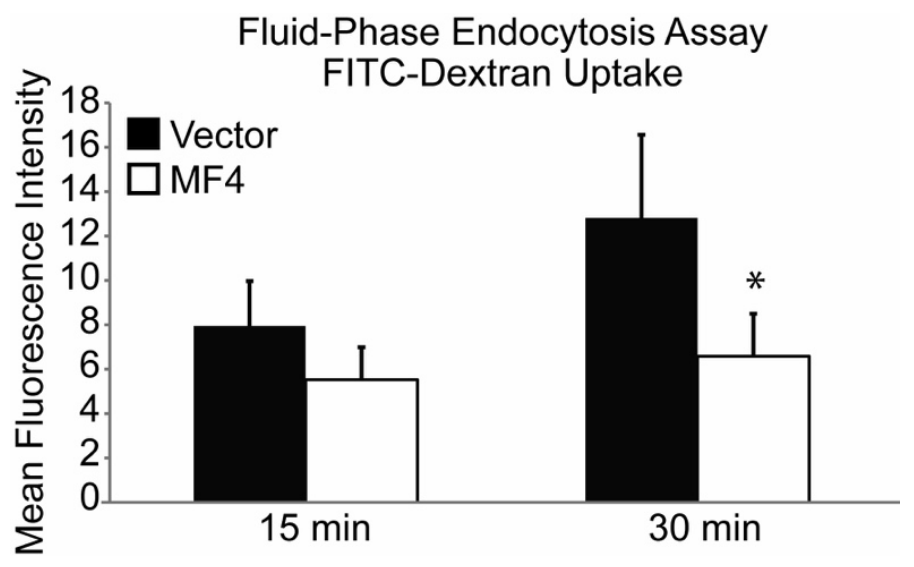

Figure S2.2: PIKfyve inhibition impairs accumulation of fluid-phase pinocytosis.

A. RAW cells were treated with either vector (control) or with $100 \mathrm{nM} \mathrm{MF4}$ for $2 \mathrm{~h}$. Cells were then allowed to pinocytose fluorescent-dextran for the indicated times, fixed and then observed by microscopy. B. Alternatively, cells were fixed and analysed by flow cytometry. Shown is the background corrected fluorescence-average of dextran per cell after counting 10,000 cells per condition per experiment for six independent experiments. The uptake of FITC-conjugated dextran was compromised in those macrophages treated with MF4 for $2 \mathrm{~h}$, as early as 15 min after the onset of dextran incubation $(p<0.03)$, and this effect was even more pronounced with 30 min pulse $(p<0.01)$. 


\section{Chapter 3}

\subsection{The phosphoinositide-gated lysosomal $\mathrm{Ca}^{2+}$ channel, TRPML1, is required for phagosome maturation}

\subsubsection{Contribution of Authors and Co-Authors}

Author: Roya Monica Dayam

contributions: Performed the experiments in figures 3.1, 3.2, 3.4 (A, B, D and E), 3.5, 3.6, 3.7, S3.1, AND S3.2. Generated figures and helped in the preparation of the manuscript.

Co-Author: Amra Saric

contributions: performed the experiment in figure 3.3.

Co-authors: Ryan E. Shilliday

Contributions: performed the experiment in figure $3.4 \mathrm{C}$.

Co-Author: Roberto Botelho

Contributions: Provided essential insight of the experiments and interpretation of the results. Wrote the manuscript.

\section{Status of the manuscript:}

Prepared for submission to a peer-reviewed journal Officially submitted to a peer-reviewed journal Accepted by a peer-reviewed journal

_ _ _ Published in a peer-reviewed journal

Published by John Wiley \& Sons Ltd

In Volume 16, Issue 9, 1010-1026 (2015) 


\title{
The phosphoinositide-gated lysosomal $\mathrm{Ca}^{2+}$ channel, TRPML1, is required for phagosome maturation
}

The following work has been submitted and published in Traffic journal

\author{
Roya M. Dayam ${ }^{1,2}$, Amra Saric $^{1,2}$, Ryan E. Shilliday ${ }^{1}$ and Roberto J. Botelho ${ }^{1,2 *}$ \\ ${ }^{1}$ Dept. of Chemistry and Biology and the ${ }^{2}$ Molecular Science Program, Ryerson \\ University, Toronto, Ontario, Canada, M5B2K3 \\ ${ }^{*}$ Corresponding author: Roberto J. Botelho, 350 Victoria Street, Ryerson University, \\ Toronto, Ontario, Canada, M5B2K3
}




\subsection{Abstract}

Macrophages internalize and sequester pathogens into a phagosome. Phagosomes then sequentially fuse with endosomes and lysosomes, converting into degradative phagolysosomes. Phagosome maturation is a complex process that requires regulators of the endosomal pathway including the phosphoinositide lipids. Phosphatidylinositol3-phosphate and phosphatiydlinositol-3,5-bisphosphate (PI(3,5)P2 ), which respectively control early endosomes and late endolysosomes, are both required for phagosome maturation. Inhibition of PIKfyve, which synthesizes PI(3,5)P2 , blocked phagosomelysosome fusion and abated the degradative capacity of phagosomes. However, it is not known how PIKfyve and PI(3,5)P2 participate in phagosome maturation. TRPML1 is a $\mathrm{PI}(3,5) \mathrm{P} 2$-gated lysosomal $\mathrm{Ca}^{2+}$ channel. Since $\mathrm{Ca}^{2+}$ triggers membrane fusion, we postulated that TRPML1 helps mediate phagosome-lysosome fusion. Using Fcy receptor-mediated phagocytosis as a model, we describe our research showing that silencing of TRPML1 hindered phagosome acquisition of lysosomal markers and reduced the bactericidal properties of phagosomes. Specifically, phagosomes isolated from TRPML1-silenced cells were decorated with lysosomes that docked but did not fuse with. We could rescue phagosome maturation in TRPML1-silenced and PIKfyveinhibited cells by forcible $\mathrm{Ca}^{2+}$ release with ionomycin. We also provide evidence that cytosolic $\mathrm{Ca}^{2+}$ concentration increases upon phagocytosis in a manner dependent on TRPML1 and PIKfyve. Overall, we propose a model where PIKfyve and PI(3,5)P2 activate TRPML1 to induce phagosome-lysosome fusion. 


\subsection{Introduction}

Macrophages are professional phagocytes of the immune system that roam around tissues to hunt and eliminate pathogens such as bacteria and fungi. Upon contact with a pathogen, receptors embedded on the macrophage plasma membrane engage cognate ligands on the pathogen surface to trigger a complex signaling network involving protein kinases, GTPases and various lipid second messengers that together coordinate the localized remodeling of the actin cytoskeleton and membrane (Flannagan, Jaumouillé, \& Grinstein, 2012; Underhill \& Ozinsky, 2002). This culminates in engulfment of the pathogen in a process known as phagocytosis. The pathogen is now sequestered in a new organelle, or phagosome. Nascent phagosomes are similar to the plasma membrane and the extracellular medium, but are quickly remodeled by sequentially fusing with endosomes and lysosomes (Fairn \& Grinstein, 2012; Otilia V Vieira, Botelho, \& Grinstein, 2002). This maturation process converts the phagosome from an innocuous organelle into a phagolysosome, endowed with hydrolases and an acidic milieu that kills and digests the pathogen (Fairn \& Grinstein, 2012; Kielian \& Cohn, 1980; Pesanti \& Axline, 1975; Otilia V Vieira et al., 2002).

As with phagocytosis, phagosome maturation is a highly coordinated process that requires the endolysosomal protein machinery that controls membrane fission, targeting and fusion, including SNARE proteins, Rab GTPases and phosphoinositide (PI) lipid signals (Fairn \& Grinstein, 2012; Otilia V Vieira et al., 2002). With respect to Pls, phosphatidylinositol-3-phosphate (Ptdlns(3)P) and phosphatidylinositol-3,5bisphosphate are implicated in phagosome maturation (Balla, 2013; C. D. Ellson, 
Anderson, et al., 2001; Fratti, Backer, Gruenberg, Corvera, \& Deretic, 2001; G. H. E. Kim, Dayam, Prashar, Terebiznik, \& Botelho, 2014; O V Vieira et al., 2001). Ptdlns(3)P predominates on early endosomes, where it helps to recruit effector proteins like EEA1, HRS and sorting nexins to mediate endosome trafficking (Birkeland \& Stenmark, 2004; Gillooly et al., 2000; Urbé, Mills, Stenmark, Kitamura, \& Clague, 2000; Xu, Hortsman, Seet, Wong, \& Hong, 2001). These effectors typically carry a FYVE or a PX domain; fusion of these domains to GFP is useful to probe Ptdlns(3)P dynamics (C. D. Ellson, Anderson, et al., 2001; C. D. Ellson, Gobert-Gosse, et al., 2001; Gillooly et al., 2000). In fact, GFP-FYVE and GFP-PX chimeric proteins were used to show that early phagosomes acquire PtdIns(3)P soon after closure, followed by depletion within 10 minutes (C. D. Ellson, Anderson, et al., 2001; G. H. E. Kim et al., 2014; O V Vieira et al., 2001). In part, depletion of Ptdlns(3)P seems to occur by conversion into PI(3,5)P2 (Hazeki et al., 2012; G. H. E. Kim et al., 2014).

$\mathrm{PI}(3,5) \mathrm{P} 2$ is thought to predominate in late endosomes/lysosomes and is synthesized by the PtdIns(3)P 5-kinase PIKfyve (de Lartigue et al., 2009; Ho, Alghamdi, \& Botelho, 2012; O C Ikonomov, Sbrissa, \& Shisheva, 2001; Ognian C Ikonomov, Sbrissa, Foti, Carpentier, \& Shisheva, 2003; Jefferies et al., 2008; McCartney, Zhang, \& Weisman, 2014). Inhibition of PIKfyve causes a dramatic enlargement of endolysosomes, appears to disrupt lysosomal recycling and may impair autophagic flux, among other defects (de Lartigue et al., 2009; Ho et al., 2012; O C Ikonomov et al., 2001; Martin et al., 2013; McCartney et al., 2014; Nicot et al., 2006; Rutherford et al., 2006). Pharmacological inhibition or siRNA-mediated gene silencing of PIKfyve affected phagosome maturation 
in macrophages (G. H. E. Kim et al., 2014). In particular, this retarded the loss of PtdIns(3)P from phagosomes, impaired phagosome-lysosome fusion and abated the proteolytic activity of the phagosome lumen (G. H. E. Kim et al., 2014). However, it is not known how PIKfyve/PI(3,5)P2 is necessary for phagosome maturation.

There are only a few known PI(3,5)P2 effectors in mammalian cells (Ho et al., 2012; McCartney et al., 2014). Interestingly, various studies suggest that PI(3,5)P2 may control multiple ion channels (Dong, Shen, Wang, Dawson, Li, Zhang, Cheng, Zhang, Weisman, Delling, \& Xu, 2010; Munoz, Almilaji, Setiawan, Föller, \& Lang, 2013; Pakladok, Almilaji, Munoz, Alesutan, \& Lang, 2013; Tsuruta, Green, Rousset, \& Dolmetsch, 2009). One of the best characterized is TRPML1 (or mucolipin-1), a lysosomal channel that facilitates diffusion of $\mathrm{Ca}^{2+}$ and other metals out of the lysosome (Dong et al., 2009; Dong, Shen, Wang, Dawson, Li, Zhang, Cheng, Zhang, Weisman, Delling, \& Xu, 2010; Wang, Zhang, Gao, \& Xu, 2014; Zeevi, Frumkin, \& Bach, 2007). The list of functions associated with TRPML1 activity is growing and include trafficking to lysosomes, heterotypic lysosome fusion, lysosome reformation and lysosome exocytosis during plasma membrane repair and phagocytosis of large particles (Cheng et al., 2014; Dong, Shen, Wang, Dawson, Li, Zhang, Cheng, Zhang, Weisman, Delling, \& Xu, 2010; Paul R Pryor et al., 2006; M. Samie et al., 2013; Shen et al., 2012; Thompson, Schaheen, Dang, \& Fares, 2007). Indeed, these endolysosomal functions overlap with those associated with endolysosomal $\mathrm{Ca}^{+2}$, including triggering heterotypic late endosome-lysosome fusion and lysosome exocytosis (Gerasimenko, Gerasimenko, \& Petersen, 2001; Kiselyov, Ahuja, Rybalchenko, Patel, \& Muallem; P R Pryor, Mullock, Bright, Gray, \& 
Luzio, 2000; Reddy, Caler, \& Andrews, 2001; M. Samie et al., 2013). Importantly, the Nterminal cytosolic tail of TRPML1 binds to PI(3,5)P2 (Dong, Shen, Wang, Dawson, Li, Zhang, Cheng, Zhang, Weisman, Delling, \& Xu, 2010). This binding increases the openprobability of TRPML1, leading to $\mathrm{Ca}^{+2}$ diffusion (Dong, Shen, Wang, Dawson, Li, Zhang, Cheng, Zhang, Weisman, Delling, \& Xu, 2010). Interestingly, tandem copies of the $\mathrm{PI}(3,5) \mathrm{P} 2$-binding region of TRPML1 encompassing residues 1-68 was recently shown to serve as a sensor of PI(3,5)P2 (Li et al., 2013).

Given the above, we speculated that TRPML1 may be required for phagosome maturation by stimulating phagosome-lysosome fusion and that this may be part of the mechanism by which PIKfyve helps control phagosome maturation. Here, we tested this hypothesis by silencing TRPML1 expression in macrophages and observing the effect on phagosome maturation. As a model, we employed antibody-coated particles, which are internalized by engaging the phagocytic Fc $\gamma$ receptors (Flannagan et al., 2012). We observe that TRPML1 silenced cells are defective for phagosome maturation. Importantly, lysosomes could dock with phagosomes but failed to merge in TRPML1-silenced cells. Importantly, $\mathrm{Ca}^{+2}$ ionophores were sufficient to rescue phagosome maturation in both TRPML1-silenced cells and PIKfyve-inhibited cells. Lastly, we provide evidence that phagocytosis induces changes in cytosolic $\mathrm{Ca}^{2+}$ in a manner dependent on PIKfyve and TRPML1 and independent of extracellular and endoplasmic reticulum-derived $\mathrm{Ca}^{2+}$. Overall, we propose a model by which PIKfyve, likely through PI(3,5)P2 activates TRPML1 to stimulate phagosome-lysosome fusion. 


\subsection{Results and Discussion}

3.4.1 TRPML1 silencing in macrophages impacts lysosome morphology

TRPML1 is a lysosomal $\mathrm{Ca}^{2+}$ channel gated by PI(3,5)P2 (Dong, Shen, Wang, Dawson, Li, Zhang, Cheng, Zhang, Weisman, Delling, \& Xu, 2010). To examine its importance towards phagosome maturation, we pursued siRNA-mediated gene silencing to eliminate TRPML1 in the RAW 264.7 macrophage cell line. After $30 \mathrm{~h}$ postelectroporation of silencing oligonucleotides, we tested the efficiency of TRPML1 silencing by two methods. First, we employed qRT-PCR to demonstrate that TRPML1 mRNA copy number was abated by $\sim 60 \%$ in cells treated with oligonucleotides against TRPML1 (siRNA ${ }^{\text {TRP }}$ ) relative to non-targeting oligonucleotides (siRNA ${ }^{\text {SC }}$ ) (Fig. 3.1A). Second, we demonstrated that TRPML1 protein expression was reduced by $\sim 65 \%$ in siRNA ${ }^{\text {TRP }}$-treated cells versus cells treated with siRNA ${ }^{S C}$ (Fig. 3.1B). To further confirm successful TRPML1 silencing and demonstrate that this impacted cells, we then examined the morphology of early endosomes and late endosomes/lysosomes (endolysosomes) by staining for EEA1 and LAMP1, respectively. As illustrated in Fig. 3.1C, TRPML1 silencing had no apparent effect on EEA1 distribution or morphology. By comparison, siRNA ${ }^{\text {TRP }}$-treatment increased the number of LAMP1-positive compartments relative to the siRNA ${ }^{\text {Sc }}$ condition, as previously observed ((Thompson et al., 2007); Fig. 3.1C, D). Overall, we can efficiently silence TRPML1 expression in RAW macrophages to examine its role in phagosome maturation. 

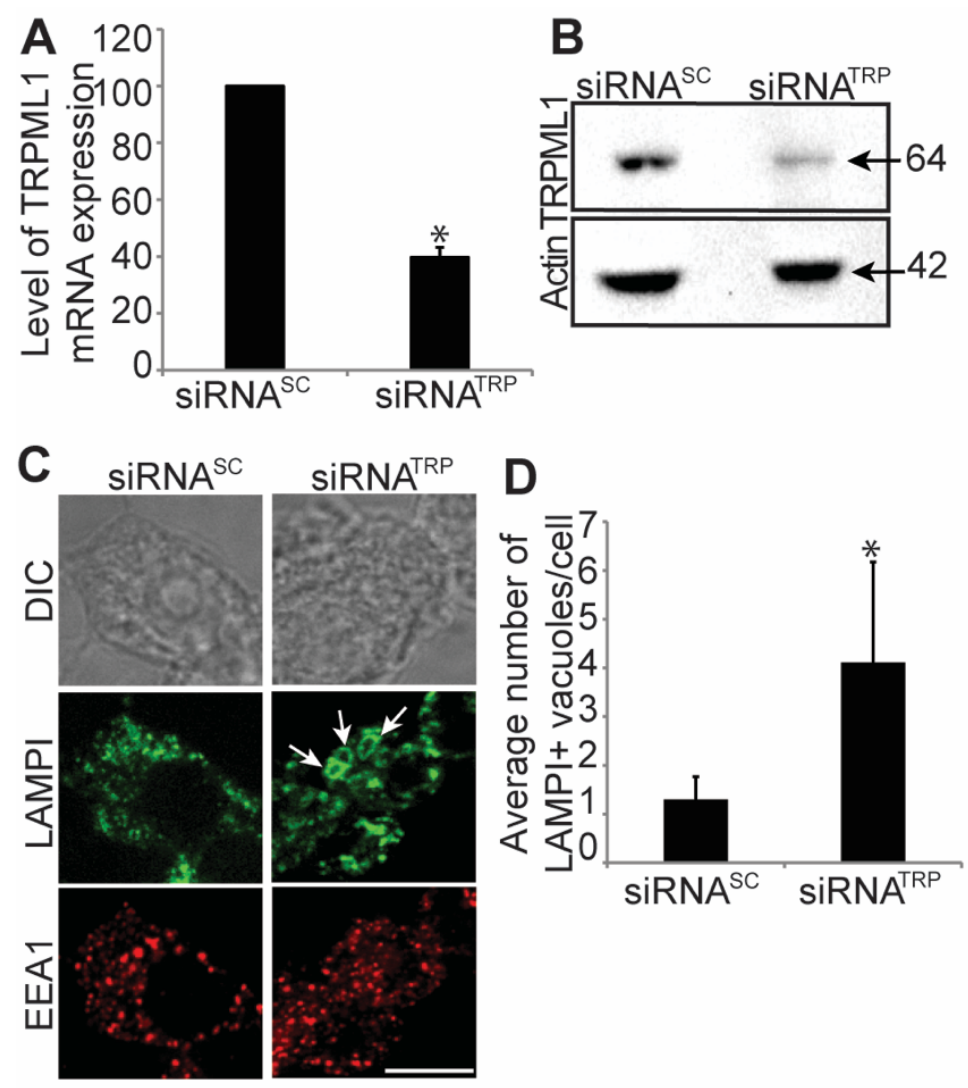

Figure 3.1: TRPML1 silencing and its effect on lysosomes.

RAW macrophages were electroporated with non-targeting siRNA (siRNA ${ }^{\mathrm{Sc}}$ ) or with TRPML1 siRNA oligonucleotides (siRNA ${ }^{T R P}$ ) as described in methods. A. The efficiency of TRPML1 silencing measured by quantitative real-time PCR after reverse transcription. Shown are the mean \pm the standard error of the mean (SEM) from at least three independent experiments, each with three replicates, and normalized to control cells. Using the Student's t-test, siRNA ${ }^{\text {TRP }}$-treated cells had significantly less TRPML1 mRNA compared to control cells $(n-3, p<0.01)$. B. The efficiency of TRPML1 silencing measured by Western blotting with anti-TRPML1 antibodies $(n=2)$. C. After electroporation and silencing, cells were stained with anti-EEA1 and anti-LAMP1 antibodies to determine the effect of TRPML1 silencing on early endosomes and lysosome distribution and morphology. While there was no apparent effect on early endosomes, TRPML1 silencing increased the number of enlarged lysosomes (arrows) relative to cells treated with siRNA ${ }^{\text {Sc }}$. D. Number of LAMP1-positive compartments $>1 \mu \mathrm{m}$ in diameter. Shown are the mean \pm SEM from at least three independent experiments. Using Student's t-test, there were significantly more swollen LAMP1 compartments in siRNA ${ }^{\text {TRP }}$-treated relative to control $(n=3, p<0.05)$. Scale bar $=10 \mu \mathrm{m}$. 


\subsubsection{TRPML1 silencing impedes phagocytosis}

To follow the role of TRPML1 in phagosome maturation, we first examined its role in Fc $\gamma$ R-mediated phagocytosis of IgG-coated beads. This was important because i) phagocytosis needs to occur to examine phagosome maturation, ii) we had previously shown that PIKfyve inhibition could handicap phagocytosis (G. H. E. Kim et al., 2014), and iii) TRPML1 was previously reported to be necessary for the engulfment of large particles (M. Samie et al., 2013). More specifically, Samie et al. showed that TRPML1 ${ }^{-1}$ macrophages had difficulty in engulfing red blood cells and beads with a $6 \mu \mathrm{m}$ diameter but were not impaired in ingesting beads $3 \mu \mathrm{m}$ in diameter (M. Samie et al., 2013). Thus, we employed latex beads with an in-between average diameter of $3.9 \mu \mathrm{m}$, expecting a partial defect. As we observed before, cells treated with $50 \mathrm{nM}$ apilimod were attenuated for phagocytosis, (Fig. 3.2A; (G. H. E. Kim et al., 2014)). Similarly, RAW cells silenced for TRPML1 exhibited a significant reduction of about $70 \%$ in particle internalization relative to cells treated with the siRNA ${ }^{S C}$ (Fig. 3.2B). Interestingly, latex particles with a smaller diameter $(2.6 \mu \mathrm{m})$ were engulfed at similar rates by $\operatorname{siRNA}^{\mathrm{Sc}}$ - and siRNA ${ }^{\text {TRP }}$-treated cells (Fig. 3.2B). Thus, and as previously observed, phagocytosis of larger beads is more sensitive to TRPML1 loss-of-function. However, the requirement for TRPML1 in phagocytosis may also depend on particle type, shape and surface ligands, as elaborated on below.

Given that TRPML1 is a PI(3,5)P2 -gated $\mathrm{Ca}^{2+}$ channel, we then tested if we could rescue phagocytosis of the $3.9 \mu \mathrm{m}$ particles by forcibly increasing the cytosolic $\mathrm{Ca}^{2+}$ concentration with ionomycin, a $\mathrm{Ca}^{2+}$ ionophore, in cells inhibited for TPRML1 or 
PIKfyve. Notably, a short 5 min treatment with ionomycin was sufficient to rescue phagocytosis in both TRPML1-silenced and PIKfyve-inhibited cells (Fig. 3.2C,D). The ionomycin-based phagocytic rescue may be explained by one of or a combination of the following two models: i) rescue may be due to a largely, non-specific exocytosis of endomembranes triggered by high $\mathrm{Ca}^{2+}$ concentration that helps complete stalled particle engulfment and/or ii) the rescue suggests that a specific $\mathrm{Ca}^{2+}$ signal fails to arise in TRPML1-silenced and PIKfyve-inhibited cells, stalling phagocytosis, likely due to attenuated secretion of endomembranes at phagocytic cups. We argue that the latter model is likely since Samie et al. had similarly observed enhancement of phagocytosis upon pharmacological activation of TRPML1 with ML1-SA, a synthetic agonist peptide of TRPML1 (M. Samie et al., 2013). In addition, several studies indicate that efficient phagocytosis, especially of larger particles, requires localized exocytosis of endomembranes, including recycling endosomes and lysosomes, a process that requires $\mathrm{Ca}^{2+}$ release from endomembranes (Bajno et al., 2000; Braun et al., 2004; Czibener et al., 2006; M. Samie et al., 2013). 

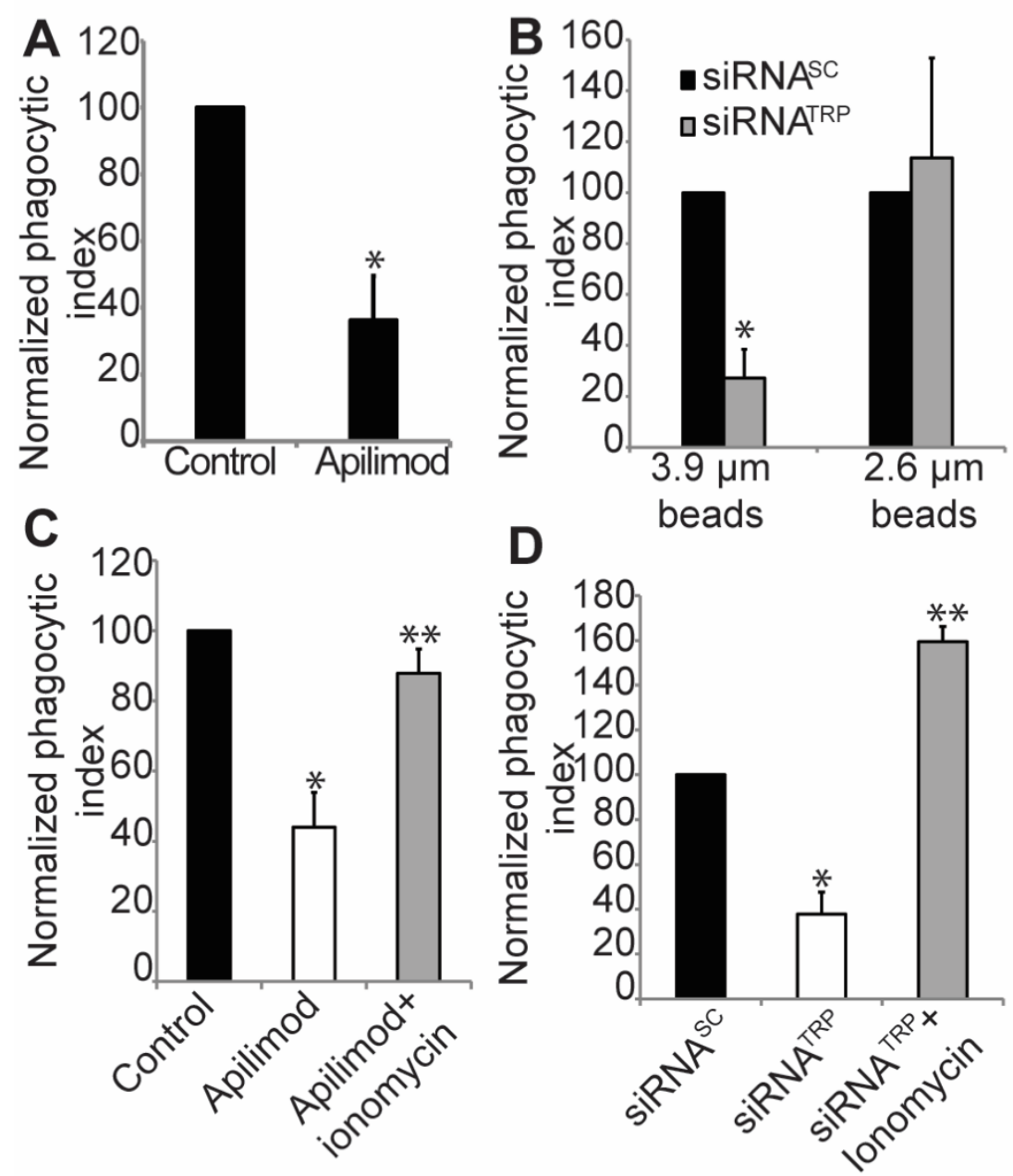

Figure 3.2: The effect of TRPML1 silencing on phagocytosis in macrophages.

A. RAW macrophages were either not treated (control) or exposed to $50 \mathrm{mM}$ apilimod for $1 \mathrm{~h}$. B. RAW macrophages were electroporated with either non-targeting siRNA $\left(\operatorname{siRNA}^{\mathrm{Sc}}\right)$ or with TRPML1 siRNA oligonucleotides $\left(\operatorname{siRNA}^{\mathrm{TRP}}\right)$. A,B. Cells were then permitted to engulf particles of a given diameter for 15 min and the phagocytic index was scored by counting the number of phagosomes in at least 100 cells per condition. C,D. Alternatively, cells were exposed to $10 \mu \mathrm{M}$ ionomycin for $5 \mathrm{~min}$ after $10 \mathrm{~min}$ of phagocytosis of $3.9 \mu \mathrm{m}$ partilcles and the phagocytic index scored as before. Shown are the mean \pm SEM from at least three independent experiments normalized to control cells. Using ANOVA test, followed by Tukey's post-hoc test, we observed a striking inhibition of phagocytosis of $3.9 \mu \mathrm{m}$ particles in apilimod and TRPML1-silenced cells relative to control (asterisks in $A-D, p<0.01$ ) and a significant rescue by ionomycin treatment relative to the respective apilimod and siRNA ${ }^{\text {TRP }}$ conditions (double asterisks in $C$ and $D ; p<0.05)$. 


\subsubsection{TRPML1 activity does not affect phosphoinositide dynamics on}

phagosomes

Using GFP-fusion proteins of FYVE and PX domains, it is well known that early phagosomes become enriched in Ptdlns(3)P immediately after closure. This enrichment is transient since the GFP-probes dissociate from phagosomes in less than $10 \mathrm{~min}$ (C. D. Ellson, Anderson, et al., 2001; O V Vieira et al., 2001). We and others had previously shown that PIKfyve antagonists affected the phagosomal kinetics of Ptdlns(3)P using 2FYVE-GFP (Hazeki et al., 2012; G. H. E. Kim et al., 2014). In particular, dissociation of the probe was significantly delayed in PIKfyve-inhibited cells. We postulated that this was related to the role of PIKfyve in converting Ptdlns(3)P into PI(3,5)P2 and predicted that TRPML1, as an effector of PIKfyve, would have little impact on phagosomal PtdIns(3)P dynamics. Therefore, we compared untreated, siRNA ${ }^{\mathrm{Sc}}$, siRNA ${ }^{\mathrm{TRP}}$ and apilimod-treated cells expressing 2FYVE-GFP and undergoing live phagocytosis. By synchronizing to the apparent time of closure and measuring the fluorescence intensity of 2FYVE-GFP on phagosomes over the cytosolic signal, we estimated the relative abundance of Ptdlns(3)P on phagosomes over time. As expected, there was little difference in the acquisition of 2FYVE-GFP in any of the treatments (Fig. 3.3A,B). In comparison, apilimod-treated cells exhibited a significant delay in the loss of 2FYVE-GFP,

which was not observed in siRNA ${ }^{\mathrm{TRP}}$-treated macrophages (Fig. 3.3A, B). Thus, we suggest that PIKfyve activity is necessary for efficient turnover of Ptdlns(3)P on phagosomes, likely by conversion to PI(3,5)P2, and that this is independent of TRPML1. 
This also suggests that TRPML1 may not play a significant role in controlling early phagosomal properties. 

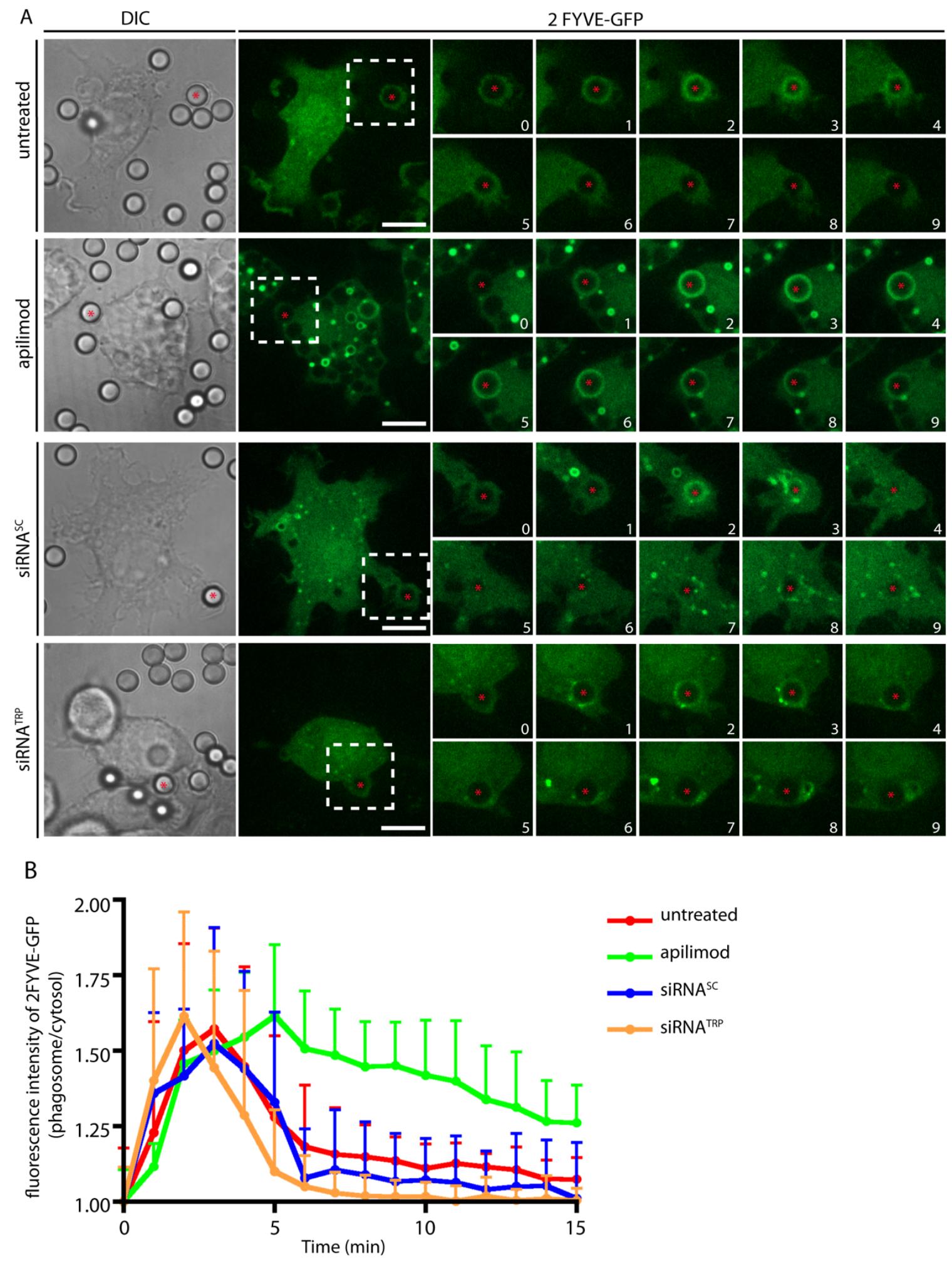
Figure 3.3: FPhosphatidylinositol-3-phosphate dynamics on phagosomes in TRPML1silenced macrophages.

A. RAW macrophages were co-electroporated with siRNA oligonucleotides and plasmid encoding 2FYVE-GFP. The left most panels show represenative DIC and fluorescence frames at time $=0$ min of time-lapse microscopy of cells undergoing phagocytosis. The square dotted box represents a magnified area shown in the right most panels. Red asterisks indicate phagosomes tracked over time. Elapsed time in minutes is indicated, where $0 \mathrm{~min}$ is the estimated time of closure. Scale bar $=10 \mu \mathrm{m}$. B. Quantification of 2FYVE-GFP fluorescence as the ratio of 2FYVE-GFP intensity on the phagosome to the cytosol. Shown are the mean ratio \pm standard deviation normalized to time "0 min". Apilimod-treated cells (green trace) exhibited slow loss of 2FYVE-GFP from phagosomes relative to the other conditions

3.4.4 TRPML1 silencing impairs phagosome maturation and bactericidal activity

Given that PIKfyve is required for phagosome maturation and that $\mathrm{PI}(3,5) \mathrm{P} 2$ gates TRPML1 activity, we postulated that TRPML1 may control phagosome maturation by facilitating fusion with endolysosomes. To test this, we permitted RAW macrophages treated with siRNA ${ }^{T R P}$ to internalize IgG-opsonized beads, followed by a $1 \mathrm{~h}$ chase to elicit phagolysosome formation. Cells were then stained for LAMP1, a canonical marker of endolysosomes. We then applied a pseudocolour filter to differentiate between strongly, intermediate and weakly labeled phagosomes, as previously done (G. H. E. Kim et al., 2014).

As shown in Figure $4 \mathrm{~A}$ and $\mathrm{B}, \sim 60 \%$ of phagosomes in untreated and siRNA ${ }^{\mathrm{SC}}$ treated cells were strongly labeled for LAMP1 (large arrows, Fig. 4A), while 15\% were weakly labeled. By contrast, cells silenced for TRPML1 showed a dramatic reduction in strongly labeled LAMP1 phagosomes ( $<20 \%$ versus $\sim 60 \%$ in control) with a corresponding increase in weakly LAMP1-labeled phagosomes (45\% versus $15 \%$ in 
control Fig. 3.4A, B). For comparison, apilimod-treated cells exhibited an even larger attenuation in LAMP1 acquisition, with $5 \%$ and $65 \%$ of phagosomes being strongly and weakly labeled for LAMP1, respectively (arrowheads, Fig. 3.4A,B). Importantly, expression of mCherry-tagged human wild-type TRPML1, which is resistant to the silencing oligonucleotides against murine TRPML1, strongly rescued phagosome maturation in siRNA ${ }^{\mathrm{TRP}}$-treated mouse macrophages (Fig. 3.4C, D). Incidentally, phagosomes were also labeled with mCherry-TRPML1 in these rescue experiments (Fig. 3.4C). In comparison, most phagosomes remained weakly labeled for LAMP1 in cells that did not express human TRPML1 or that were transfected with mCherry-only expressing plasmids (Fig. 3.4C,D). This strongly supports that the defect in phagosome maturation observed in siRNA ${ }^{\text {TRP }}$-treated cells results from TRPML1 suppression rather than an off-target effect.

These data suggested that phagosomes failed to acquire a bactericidal milieu when PIKfyve or TRPML1 are blocked. To test this, we used the treatments above and allowed macrophages to internalize live IgG-opsonized E. coli for $1 \mathrm{~h}$, followed by $30 \mathrm{~min}$ in the presence of gentamycin to kill extracellular bacteria. Macrophages were then Iysed immediately ( $0 \mathrm{~h}$ chase) to estimate the number of bacteria engulfed, or incubated for an additional $2 \mathrm{~h}$ to estimate the rate of bacterial killing relative to $0 \mathrm{~h}$.

First, we observed $\sim 25 \%$ fewer colonies in apilimod-treated and TRPML1silenced cells relative to the respective controls at $0 \mathrm{~h}$, implying that the uptake of $E$. coli was decreased in PIKfyve- and TRPML1-inhibited cells (Supplementary Fig. S3.1). This suggests that the type of particle and/or ligands, not just the size, can affect the 
sensitivity of phagocytosis to the loss of TRPML1 function. Second, after $2 \mathrm{~h}$ of phagosome maturation, all conditions exhibited fewer surviving bacteria relative to $0 \mathrm{~h}$, illustrating that bacteria were being killed (Supplementary Fig. S3.1). However, there was a striking hindrance in the ability of PIKfyve- and TRPML1-supressed cells to kill bacteria relative to control conditions. Whereas $15-20 \%$ of bacteria remained alive after $2 \mathrm{~h}$ of phagosome maturation in control cells, this escalated to $35-40 \%$ in PIKfyve and TRPML1-deficient cells (Fig. 3.4E). These observations are consistent with our previous observation that phagosomes in PIKfyve-inhibited cells had reduced cathepsin D and impaired proteolytic activity (G. H. E. Kim et al., 2014).

Overall, our data support a model by which PIKfyve and TRPML1 are necessary for phagosome-lysosome fusion and for phagosomes to efficiently acquire a degradative milieu. This also poses the possibility that pathogens like Mycobacteria may interfere with PIKfyve and TRPML1 function to usurp the cellular machinery (Scott, Botelho, \& Grinstein, 2003). Indeed, Mycobacteria is known to disturb Ptdlns(3)P metabolism to impair phagosome maturation (Vergne et al., 2004, 2003) - thus, direct or indirect interference of $\mathrm{PI}(3,5) \mathrm{P} 2$ function is conceivable. 
A
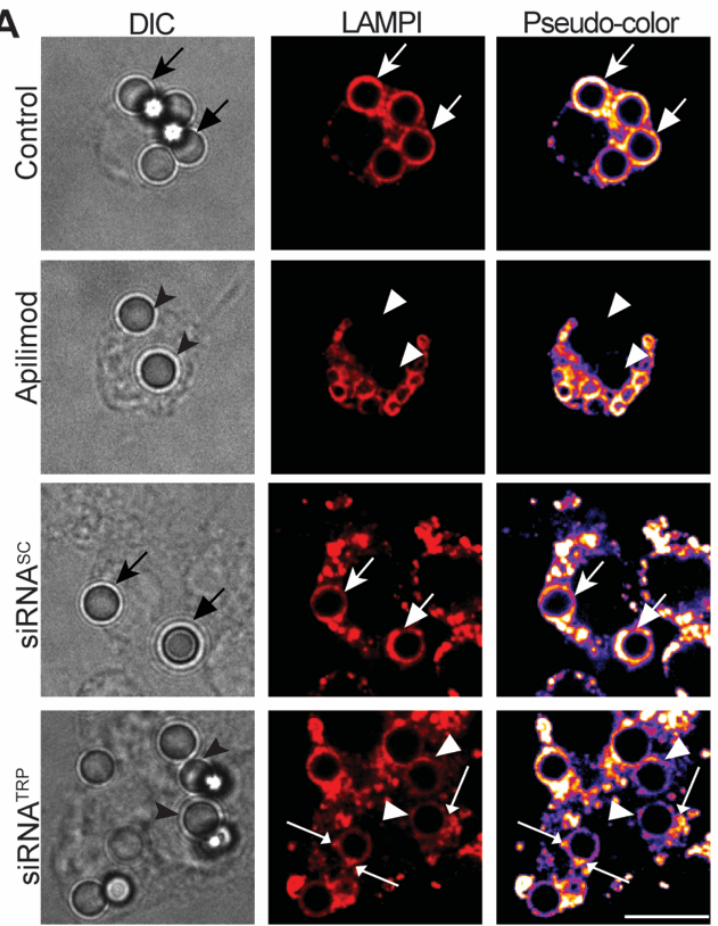

D

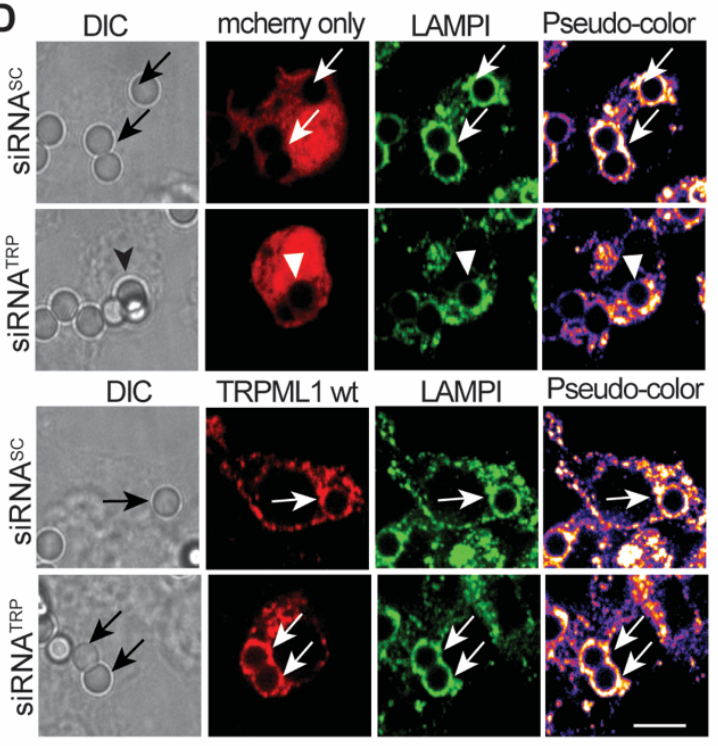

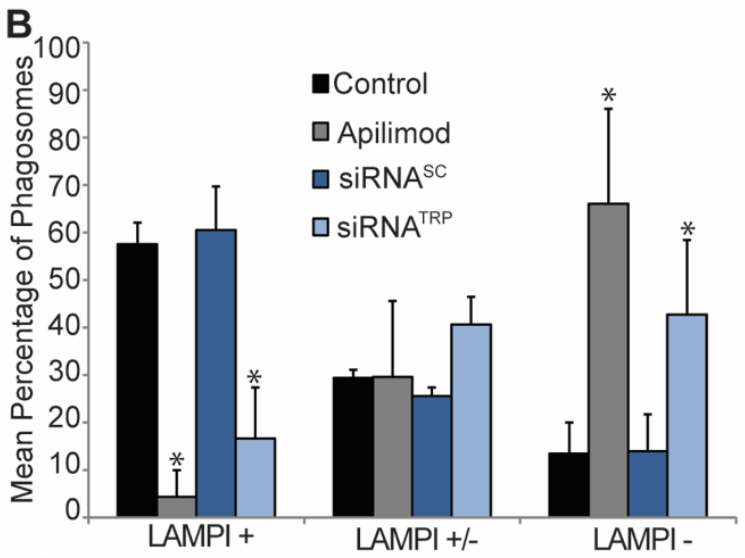

E
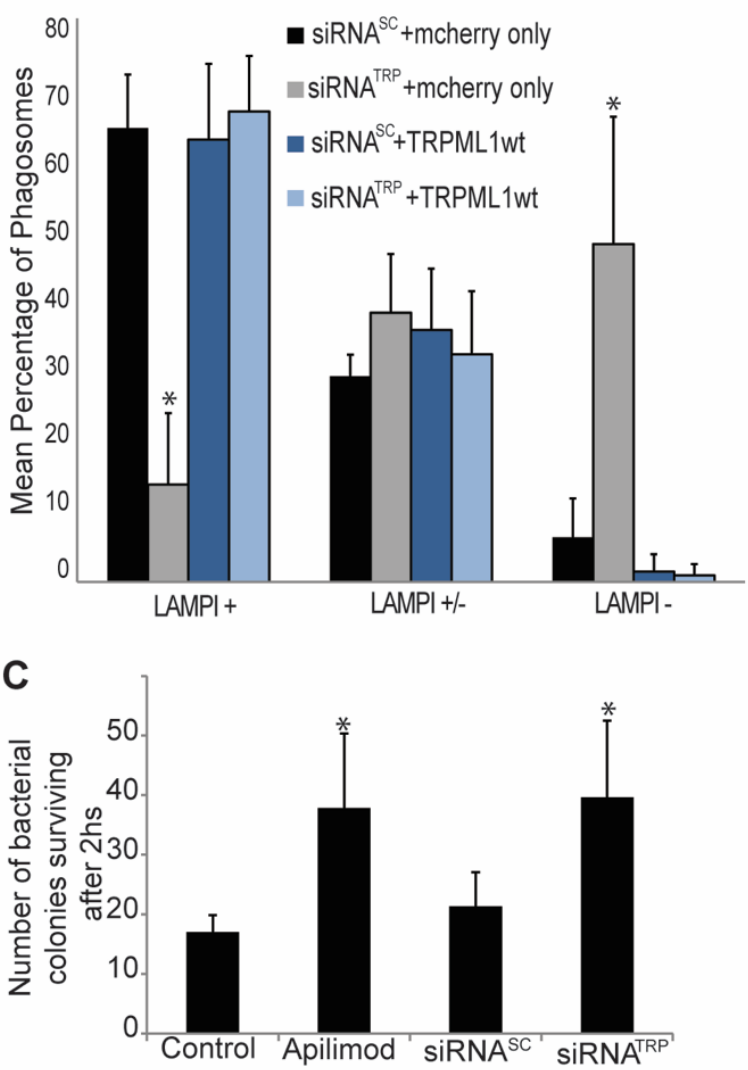

Figure 3.4: The effect of TRPML1 and PIKfyve loss of function on phagosome maturation and bacterial killing.

Cells were either untreated (control), exposed to $25 \mathrm{nM}$ apilimod, or electroporated with either siRNA ${ }^{\mathrm{Sc}}$ or siRNA ${ }^{\mathrm{TRP}}$ oligos. Cells were then allowed to phagocytose and undertake phagosome maturation as described in text. A: Micrographs showing corresponding DIC images of cells and internalized beads and LAMP1 fluorescence signal. To better assess fluorescence intensity, images were converted to pseudocolour to represent strong LAMP1 acquisition (LAMP1 ${ }^{+}$, white-yellow), intermediate LAMP1 
decoration (LAMP1 ${ }^{+/}$, orange-red) and weak LAMP1 staining (LAMP1-, blue-black). Large arrows and arrowheads point to phagosomes strongly or weakly decorated with LAMP1 staining, respectively. Thin arrows point to LAMP1 puncta/clusters associated with phagosomes. B. The percentage of phagosomes stained strongly, intermediate or weakly with LAMP1 based on at least 100 phagosomes per condition per experiment across at least six experiments. Shown is the mean percent of phagosomes in each LAMP1-staining category \pm SEM. Using one-way ANOVA test, followed by Tukey's posthoc test, there was a significant difference in the number of phagosomes stronglylabelled or weakly-labelled with LAMP1 between control/siRNA ${ }^{\text {SC }}$ cells versus apilimod or siRNA ${ }^{\text {TRP }}$-treated cells (asterisks; $p<0.05$ ). C. As described above for $A$, but cells were transfected with mCherry-only or mCherry-tagged human TRPML1 after electroporation of silencing oligonucleotides. D. The percentage of phagosomes stained strongly, intermediate or weakly for LAMP1 as described and tested above in B. E. The percent of surviving bacteria. This percent normalizes the number of colonies formed in macrophages chased for $2 \mathrm{~h}$ after engulfment to the number of colonies formed with no chase time after phagocytosis. Using paired Student's t-test, there was a significant increase in the number of colonies formed from apilimod or siRNA ${ }^{\text {TRP }}$-treated cells relative to respective controls (asterisks; $p<0.05$ ). Scale bar $=10 \mu \mathrm{m}$.

\subsubsection{Calcium suffices to rescue TRPML1 and PIKfyve defects}

Since TRPML1 was required for phagosome maturation, we asked if forcibly increasingly cytosolic $\mathrm{Ca}^{2+}$ concentration in apilimod-treated or TRPML1-silenced cells would rescue phagosome acquisition of LAMP1. Using our previous imaging analysis, we compared phagosome acquisition of LAMP1 in cells with or without a 5 min exposure to $10 \mu \mathrm{M}$ ionomycin administered $30 \mathrm{~min}$ post-phagocytosis and chased for $25 \mathrm{~min}$ after ionomycin using the conditions in Figure 3.4. As before, there was a significant reduction in the number of phagosomes strongly-labeled for LAMP1 and a corresponding increase in the number of phagosomes weakly-stained for LAMP1 in apilimod- or siRNA ${ }^{T R P}$-treated cells relative to control and siRNA ${ }^{\text {Sc }}$-treated cells (Fig. 3.5A-C). Strikingly, ionomycin treatment caused a statistically significant rebound in the number of phagosomes that were strongly labeled with LAMP1 and a corresponding 
decline in the number of phagosomes weakly stained for LAMP1 in apilimod-treated and TRPML1-silenced cells (Fig. 3.5A-C).

Since ionomycin non-specifically facilitates $\mathrm{Ca}^{2+}$ diffusion across membranes, we also wanted to confirm that endogenous $\mathrm{Ca}^{2+}$ plays a role in phagosome-lysosome fusion. To do this, we pre-incubated cells with BAPTA-AM to chelate $\mathrm{Ca}^{2+}$ during phagosome maturation. In doing this, we observed attenuated number of phagosomes strongly labeled for LAMP1 and a jump in the number of phagosomes poorly staining for the lysosomal marker (Fig. 3.5D). These observations argue that endogenous $\mathrm{Ca}^{2+}$ release is necessary for efficient phagolysosome biogenesis. 

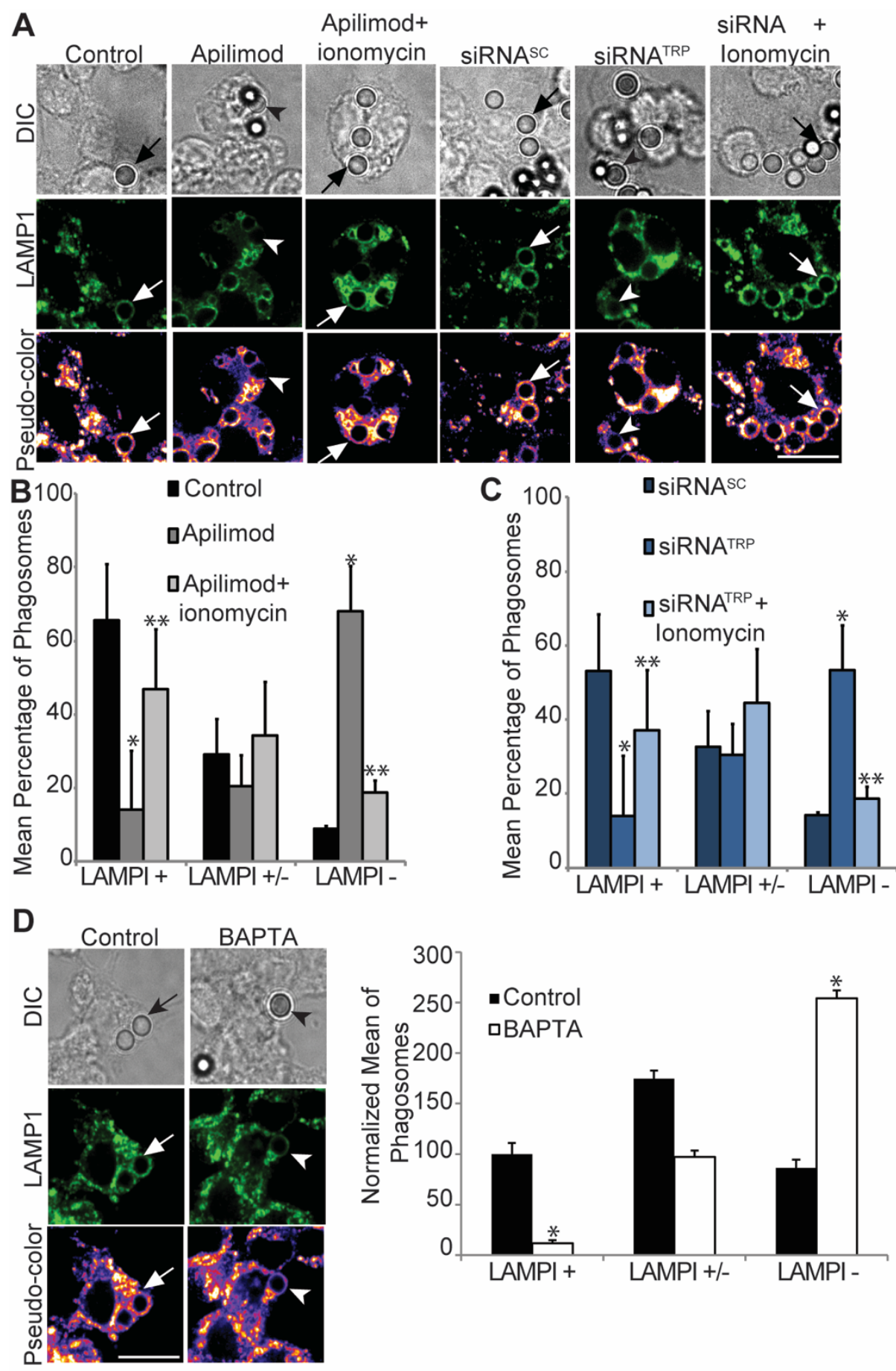

Figure 3.5: The effects of ionomycin and BAPTA-AM on phagosome acquisition of LAMP1 in PIKfyve and TRPML1-deficient cells.

A-C: Cells were either untreated (control), exposed to $25 \mathrm{nM}$ apilimod or to siRNA ${ }^{\mathrm{Sc}}$ or siRNA ${ }^{T R P}$, followed by phagocytosis and phagosome maturation, followed by sham or ionomycin-treatments as described in text. A: Micrographs of LAMP1-stained cells as described in Figure 4. Arrows and arrowheads point to phagosomes strongly or weakly 
decorated with LAMP1 staining, respectively. Scale bar $=10 \mu \mathrm{m}$. B,C: The mean percent of phagosomes \pm SEM stained strongly, intermediate or weakly with LAMP1 based on at least 100 phagosomes per condition per experiment across at least three experiments. Using one-way ANOVA and Tukey's post-hoc test, single asterisks indicate statistically important difference between control and apilimod and between control and TRPML1silenced phagosomes strongly and weakly-labelled for LAMP1 $(p<0.05)$. Double asterisks indicate statistical difference between ionomycin-treated and the respective non-ionomycin conditions $(p<0.05)$. D. Cells were either untreated or pre-incubated with BAPTA-AM before phagocytosis and LAMP1 staining as above. Normalized mean number of phagosomes \pm SEM stained strongly, intermediate or weakly for LAMP1. Asterisks indicate statistically important difference between control and BAPTA using paired Student's t-test $(p<0.05)$.

\subsubsection{TRPML1 and $\mathrm{Ca}^{2+}$ facilitate phagosome-lysosome fusion}

To complement our imaging analysis, we decided to employ cytometry to quantify

LAMP1 signal associated with isolated phagosomes. We compared phagosomes isolated from untreated cells, cells electroporated with siRNA ${ }^{\text {Sc }}$ or siRNA ${ }^{\text {TRP }}$ oligonucleotides or cells exposed to apilimod. We also compared phagosomes from the last two conditions exposed to ionomycin as performed in Figure 3.5. The relative average number of phagosomes possessing LAMP1 signal above background from each condition is shown in Figure 3.6A and 3.6B. As expected, apilimod-treated cells had fewer phagosomes stained with LAMP1 relative to control cells (Fig. 3.6A). Ionomycin treatment appeared to partially rescue the number of phagosomes containing LAMP1, consistent with our microscopy analysis.

Surprisingly, we did not observe a difference in the number of phagosomes stained for LAMP1 isolated from cells electroporated with siRNA ${ }^{\text {SC }}$, siRNA ${ }^{\text {TRP }}$ or siRNA ${ }^{\text {TRP }}$ exposed to ionomycin (Fig. 3.6B). These results were contradictory to our microscopic analysis in Figures 3.4 and 3.5. However, upon more careful inspection of our 
micrographs, we noted differences in the distribution of LAMP1-staining of phagosomes between the four treatments above. Phagosomes in control and siRNA ${ }^{\mathrm{Sc}}$-treated cells were more evenly stained with LAMP1, forming full rings around the beads (Fig. 3.4A, larger arrows). In contrast, apilimod-treated cells appeared to possess phagosomes that tended to be devoid of any LAMP1 signal, either as rings, punctate or clusters proximal to phagosomes (Fig. 3.4A, arrowheads). This was strikingly distinct from phagosomes in TRPML1-silenced cells, which despite being weaker in overall LAMP1 staining, were more often associated with LAMP1-positive puncta or clusters (smaller arrows, Fig. 3.4A). We postulated that these punctate structures were lysosomes docked to phagosomes but which failed to fuse in the absence of TRPML1.

To test this hypothesis and reconcile our flow cytometry and microscopy analyses, we performed microscopy of isolated phagosomes from various conditions above. We deduced that lysosomes docked onto phagosomes would survive the isolation process. Indeed, while phagosomes from control and siRNA ${ }^{\text {Sc }}$ cells tended to be uniformly stained for LAMP1, phagosomes from TRPML1-silenced cells were more often associated with punctate LAMP1 staining (Fig. 3.6C, arrows). Phagosomes from apilimod-treated cells were most often devoid of signal or had few LAMP1 puncta associated with them (Fig. 3.6C). Importantly, the punctate LAMP1 distribution on phagosomes was generally lost when isolated from apilimod or siRNA ${ }^{\text {TRP }}$ cells treated with ionomycin (Fig. 3.6C).

Overall, these observations are consistent with the notion that TRPML1 helps induce phagosome-lysosome fusion after docking by facilitating $\mathrm{Ca}^{2+}$ diffusion out of 
lysosomes. In addition, we suggest that PIKfyve acts upstream of TRPML1, possibly coordinating targeting of phagosomes to lysosomes and subsequently helping to activate TRPML1 to trigger phagosome-lysosome fusion. This model is consistent with various observations including i) that fast acting chelators like BAPTA-AM, but not slowacting chelators like EGTA-AM, impair heterotypic lysosome fusion (Fairn \& Grinstein, 2012; P R Pryor et al., 2000); ii) that BAPTA-AM treatment can impair phagosomelysosome fusion (Vergne et al., 2004); iii) and that pathogens like Mycobacterium actively suppress $\mathrm{Ca}^{+2}$ signals (Z A Malik, Denning, \& Kusner, 2000). In fact, Mycobacteria suppression of $\mathrm{Ca}^{2+}$ signaling is essential to maintain viability within macrophages by inhibiting calmodulin signaling and preventing phagosome uncoating (Jayachandran et al., 2007; Zulfiqar A Malik et al., 2003; Vergne et al., 2003), and that incubation with $\mathrm{Ca}^{2+}$ ionophores reduces this viability (Z A Malik et al., 2000).

The exact mechanisms that coordinate PIKfyve, TRPML1 and $\mathrm{Ca}^{2+}$ during phagosome maturation remain to be determined. For instance, while nascent phagosomes containing larger particles acquire TRPML1 by lysosome exocytosis during the internalization process (M. Samie et al., 2013), smaller phagosomes likely acquire TRPML1 only after the first round of lysosome fusion, many minutes after particle enclosure. Thus, TRPML1 may work bidirectionally to mediate phagosome-lysosome fusion in large phagosomes, but unidirectionally in smaller phagosomes. Additionally, it is unclear how PIKfyve, $\mathrm{PI}(3,5) \mathrm{P2}$ and TRPML1 are coordinated to mediate $\mathrm{Ca}^{2+}$ diffusion during phagosome-lysosome docking. Since there is constitutive $\mathrm{PI}(3,5) \mathrm{P} 2$ in lysosomes but TRPML1 is not constitutively open, we speculate that a docking sensor may 
coordinate this process. Perhaps, partial SNARE pairing may communicate to PIKfyve to locally hyper-stimulate $\mathrm{PI}(3,5) \mathrm{P} 2$ synthesis, leading to localized TRPML1 activation and $\mathrm{Ca}^{2+}$ release, which in turn reinforces SNARE binding and eventual fusion (Braun et al., 2004; Czibener et al., 2006). Such a flow of information has the potential to act as a local positive feedback loop that catalyses phagosome-lysosome fusion. Significantly, phagosomes may prove to be indispensable tools to test these hypotheses given that they are easy to isolate and their large size permits visual resolution of organelleorganelle docking and membrane subdomains as depicted in Fig. 3.4, 5 and 3.6.
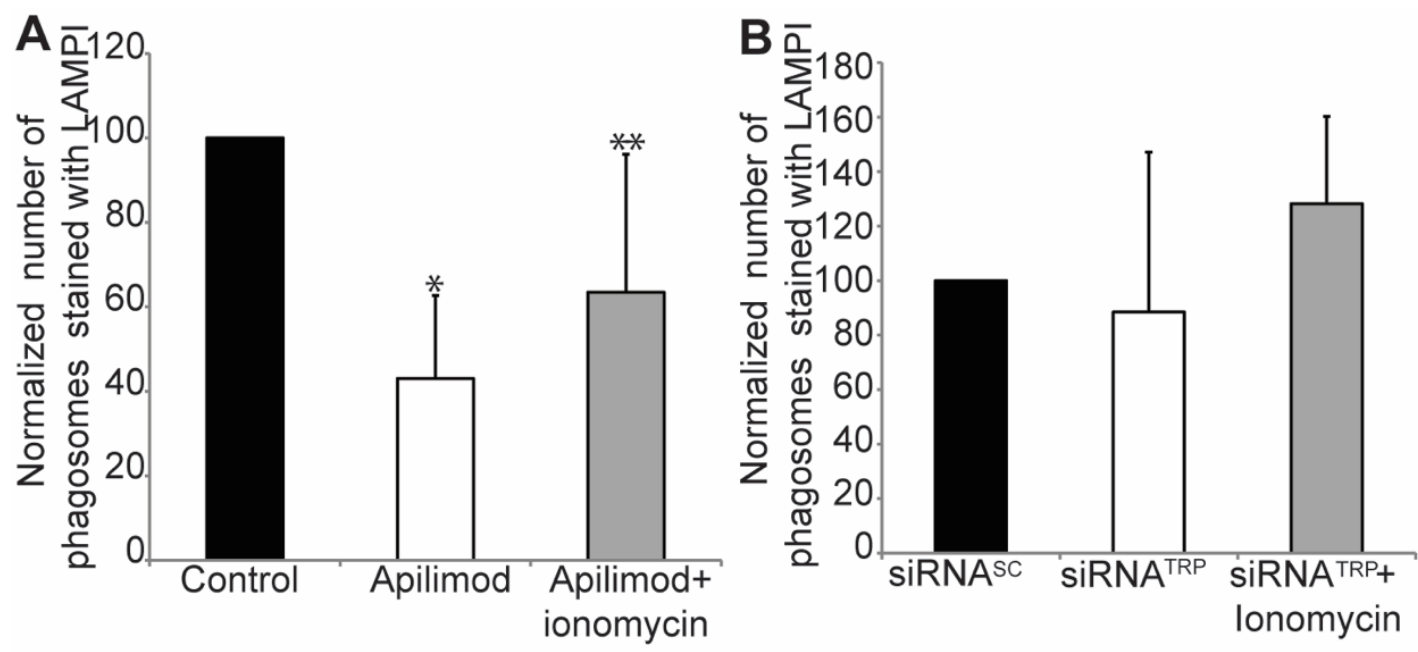

C
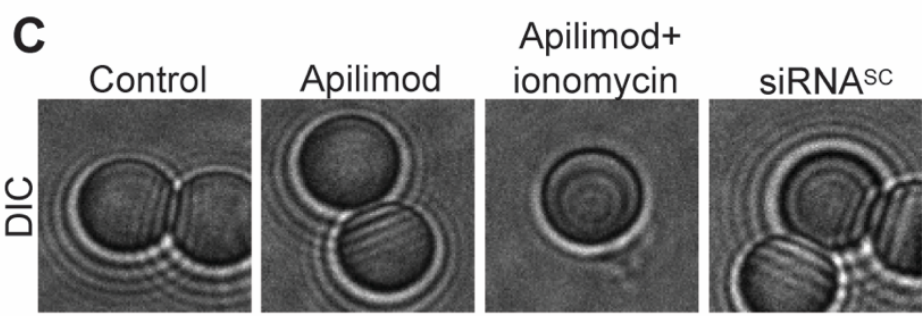

SiRNATRP+
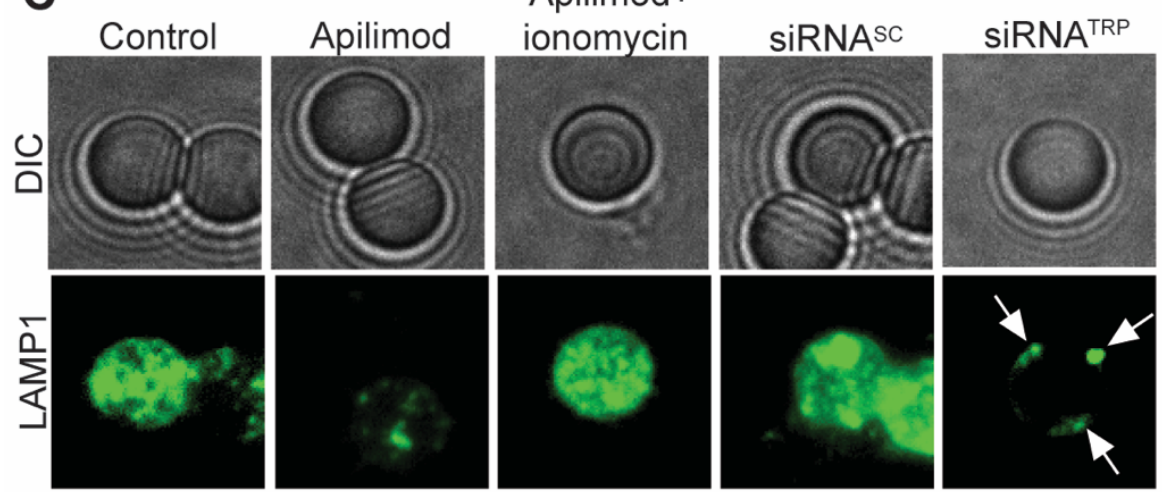

lonomycin
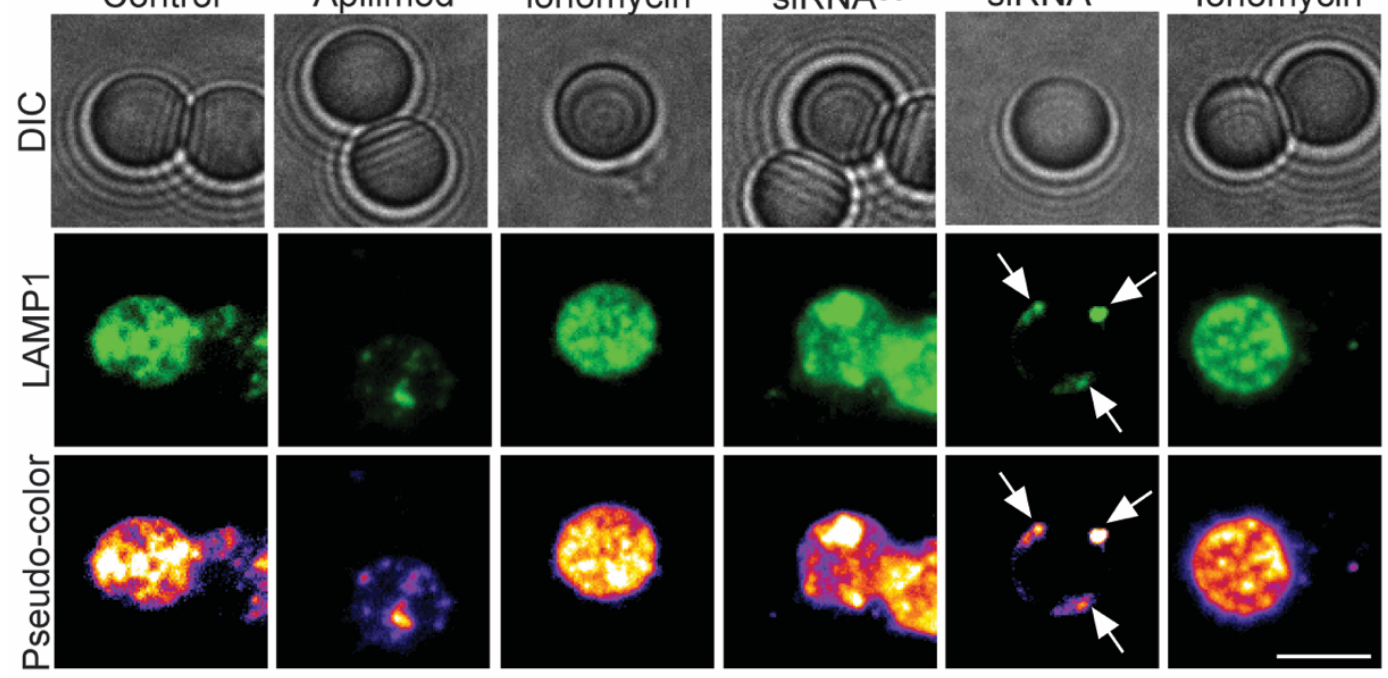
Figure 3.6: Flow cytometry and microscopy of isolated phagosomes stained for LAMP1.

RAW macrophages were treated as previously and allowed to form and mature phagosomes as in Figure 5. However, phagosomes were then isolated and stained for LAMP1. A,B: The normalized relative number of phagosomes containing LAMP1 from cells treated as indicated using flow cytometry. Mean \pm SEM are based on three independent experiments. A. Using one-way ANOVA and Tukey's post-hoc test, there was a significant difference between control and apilimod $\left({ }^{*}\right)$. Although there was no statistical difference between apilimod and apilimod plus ionomycin conditions, there was a trend towards stronger LAMP1 staining in ionomycin-treated cells. B. There was no difference between control, TRPML1-silenced with or without ionomycin using ANOVA. C. Collapsed stacks acquired using spinning disc confocal microscopy of isolated phagosomes stained for LAMP1. Phagosomes from TRPML1-silenced cells were heavily decorated with LAMP1 puncta (arrows) suggesting lysosomes docked but did not fuse with phagosomes. Phagosomes from apilimod-treated cells were often devoid of signal or decorated with fewer puncta. All other conditions displayed a more uniform distribution of LAMP1 suggesting phagosome-lysosome fusion. Scale bar $=5 \mu \mathrm{m}$.

\subsubsection{Calcium dynamics during phagosome maturation}

We next attempted to visualize live $\mathrm{Ca}^{2+}$ dynamics in macrophages undergoing phagosome maturation by using a high-efficiency $\mathrm{Ca}^{2+}$ fluorescent probe, Fluo4-AM. Treatment of macrophages with Fluo4-AM led to strongly labelled puncta and very low cytosolic signal (Fig. 3.7A). First, we determined that the puncta mostly corresponded to lysosomes by a high-degree of colocalization between Fluo4 puncta and dextranloaded lysosomes (Pearson's factor, 0.5; Fig. 3.7A). Second, we showed that ionomycin exposure led to a large increase in Fluo4 fluorescence in the cytosol, showing that the probe was present but non-fluorescent in resting macrophages (Fig. 3.7B). Thus, resting macrophages have low cytosolic but a high lysosomal $\mathrm{Ca}^{2+}$ concentration. The fact that Fluo4-AM does not label other compartments like the endoplasmic reticulum and 
mitochondria may reflect low levels of esterases needed to cleave off the acetyloxymethyl ester moiety in these organelles.

We next attempted to visualize live-cell $\mathrm{Ca}^{2+}$ dynamics during phagosome maturation by tracking Fluo4 fluorescence. Unfortunately, we could not convincingly observe localized bursts of Fluo4 fluorescence in the cytosol that might indicate a localized increase in $\mathrm{Ca}^{2+}$ concentration at sites of lysosome-phagosome fusion. This may be due to transient and non-synchronous nature of phagosome-lysosome fusion and of $\mathrm{Ca}^{2+}$ diffusion - in fact, it has been estimated that $\mathrm{Ca}^{2+}$ release occurs within $1 \mathrm{~ms}$ over a $20 \mathrm{~nm}$ region (Burgoyne \& Clague, 2003; Fairn \& Grinstein, 2012; Hay, 2007). However, we speculated that in cells with phagosomes that sufficient $\mathrm{Ca}^{2+}$ may be released from lysosomes into the cytosol to be measurable. To test this hypothesis, we measured the Fluo4 fluorescence in the cytosol, in lysosomes and the ratio of these two values.

First, we compared resting cells to macrophages undergoing phagocytosis and we found that the latter tended to have higher cytosolic Fluo4 fluorescence in the cytosol (Fig. 3.7C, Supplementary Fig. S3.2). This was accompanied by a corresponding decrease in Fluo4 fluorescence in puncta (Supplementary Fig. S3.2). Thus, when we took the ratio of Fluo4 fluorescence in the cytosol against puncta, we observed a significant increase in the ratio of cytosol-to-puncta Fluo4 signal (Fig. 3.7D). Strikingly, silencing of TRPML1 or PIKfyve inhibition prevented these changes in Fluo4-AM fluorescence; specifically, there was no increase in cytosolic Fluo4-AM fluorescence, no decrease in punctate-associated Fluo4-AM fluorescence and consequently no change to 
the fluorescence ratio of cytosol-to-puncta (Fig. 3.7C,D, Supplementary Fig. S3.2). Chelation of extracellular $\mathrm{Ca}^{2+}$ with EGTA, or pre-incubation with xestospongin $\mathrm{C}$, a selective inhibitor of $\mathrm{IP}_{3}$-gated $\mathrm{Ca}^{2+}$ channel in the endoplasmic reticulum, did not prevent the changes in Fluo4-AM fluorescence induced by phagocytosis (Fig. 3.7E, 3.7F). Overall, we interpret these data to show that TRPML1 and PIKfyve play a role in releasing $\mathrm{Ca}^{2+}$ from lysosomes to help trigger phagosome-lysosome fusion.

It remains possible that $\mathrm{Ca}^{2+}$ may also be released from other endomembrane structures, though this does not appear to be extracellular or endoplasmic reticulumsourced. We speculate that in addition to participating in phagosome-lysosome fusion, that this change in cytosolic $\mathrm{Ca}^{2+}$ concentration may act as a signal to change the activation state of macrophages. For example, this may lead to activation of calmodulin and effects on transcription factors controlling gene expression (Attout, Floto, \& Launay, 2014). Thus, we propose that $\mathrm{Ca}^{2+}$ originating from lysosome-stores may have a very important role in re-programming macrophages after phagocytosis and that this depends on PIKfyve and TRPML1. 

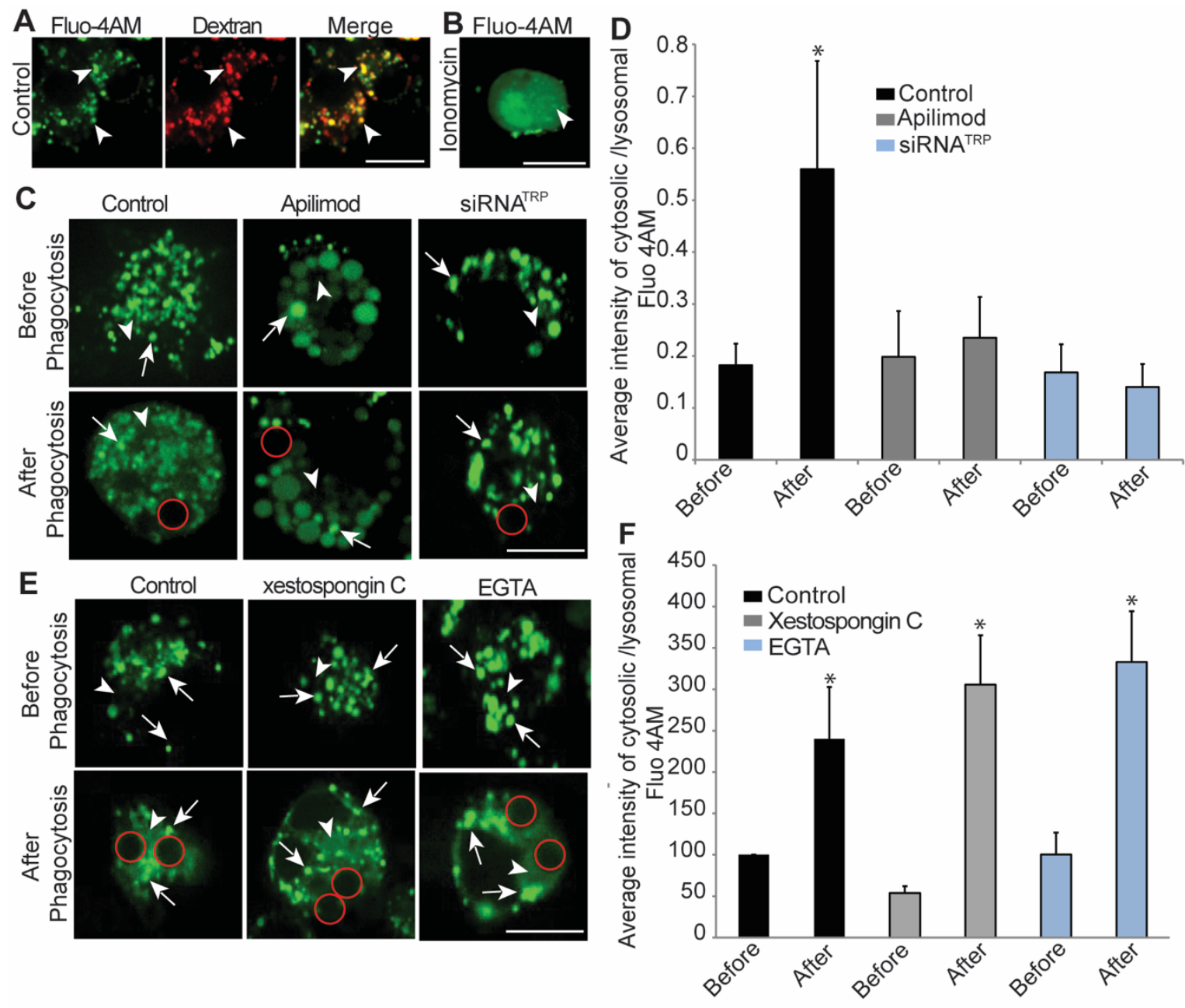

Figure 3.7: The effect of phagocytosis on Fluo4-AM fluorescence in RAW macrophages.

A. RAW macrophages were permitted to endocytose Alexa555-dextran to label lysosomes (red), followed by staining with Fluo4-AM (green) as described in text. Merge displays the overlapped channels to show co-localization of dextran and Fluo4 (arrowheads show overlapping puncta). B. RAW cells labeled with Fluo4-AM were subjected to ionomycin for 5 min to increase cytosolic $\mathrm{Ca}^{2+}$ concentration, causing high cytosolic Fluo4 fluorescence. C and E. RAW macrophages were stained with Fluo-4-AM, pre-incubated with the indicated drugs, and then imaged before phagocytosis (top panels) or at least $30 \mathrm{~min}$ after phagocytosis (bottom panels). Arrows point to Fluo4AM labeled puncta and arrowheads point to a cytosolic area. Red circles identify phagosomes. D, F. The average ratio of Fluo4-AM fluorescence intensity in cytosol to puncta \pm SEM from three independent experiments. This is based on at least 10 lysosome readings per cell, with least six cells per condition per experiment. Using oneway ANOVA, followed by Tukey's post-hoc test, we found that the fluorescence ratio of cytosol to lysosome from control, EGTA- and xestospongin C-treated cells after 
phagocytosis was statistically higher and different from all other treatments $(p<0.01)$.

Scale bar $=10 \mu \mathrm{m}$.

\subsection{Materials and Methods}

\subsubsection{Nucleic acids and antibodies}

The plasmids encoding mCherry, 2FYVE-GFP and mCherry-tagged wild-type human TRPML1 were previously characterized (Li et al., 2013; Paul R Pryor et al., 2006; O V Vieira et al., 2001). Plasmids were extracted and purified using E.Z.N.A. ${ }^{\circledR}$ Plasmid Midiprep Kit (Omega Bio-Tek) as per manufacturer's instructions. For TRPML1 gene silencing by siRNA, we used SMARTpool containing four oligonucleotides and the corresponding non-targeting oligonucleotide sets (Thermofisher, Canada, ON). For immunofluorescence, the primary antibodies used in this study were rabbit anti-mouse EEA1 (used at 1:400, Cell Signaling, MA), rat anti-mouse LAMP1 monoclonal antibodies (clone 1D4B used at 1:100, Developmental Hybridoma Bank, IA) and rabbit anti-E. coli antibodies (ABD Serotec, Raleigh, NC). For Western blotting, we employed goat antiactin antibodies and rabbit anti-TRPML1 antibodies used at a dilution of 1:1000 (Fisher Scientific, ON). Fluorescent secondary antibodies used were at 1:200 to 1:1000 (Bethyl Laboratories, TX or Life Technologies, ON) while horseradish peroxidase-linked second antibodies were used at 1:5000 to 1:10000 (Cedarlane, ON).

\subsubsection{Cell culture, transfection and gene silencing}

RAW264.7 macrophages were cultured in Dulbecco's modified Eagle's medium (DMEM) supplemented with high glucose, L-glutamine, sodium pyruvate, and $5 \%$ heat- 
inactivated fetal bovine serum (Gibco) at $37{ }^{\circ} \mathrm{C}$ in $5 \% \mathrm{CO}_{2}$. Experiments at ambient $\mathrm{CO}_{2}$ required using HEPES-buffered RPMI.

Before transfection, cells were cultured onto glass coverslips to reach $40-50 \%$ confluence. Transfection of plasmids was done using Fugene HD (Promega) as per manufacturer's instructions. For siRNA-mediated gene silencing, cells were cultured onto T25 flasks to about $90 \%$ confluence. One round of electroporation of cells with SMARTpool oligonucleotides was done by an Amaxa system, followed by incubation in DMEM plus $5 \%$ fetal bovine serum for $30 \mathrm{~h}$. For rescue experiments, cells were transfected with mCherry-tagged human TRPML1 using FuGene HD, $8 \mathrm{~h}$ postelectroporation of siRNA oligonucleotides. Co-transfection of siRNA and 2FYVE-GFP plasmid was conducted using the Neon Transfection System (Life Technologies, ON). Briefly, cultured RAW 264.7 cells were scraped and washed in PBS. Cells were resuspended in R buffer (Invitrogen) at 200,000 cells per $100 \mu \mathrm{L}$ of buffer, with $1 \mu \mathrm{g}$ of 2FYVE-GFP plasmid DNA and $20 \mathrm{nM}$ siRNA ${ }^{\text {SC }}$ or siRNA ${ }^{\text {TRP }}$ oligonucleotides. Transfection was conducted using $100 \mu \mathrm{L}$ Neon tips at 1400V, $30 \mathrm{~ms}$ width and 1 pulse, followed by plating onto coverslips with DMEM supplemented with 5\% FBS. Live cell imaging and phagocytosis was conducted after 30 hours. To quantify TRPML1-silencing, we employed quantitative real-time PCR (qRT-PCR) after reverse transcription of mRNA using SuperScript ${ }^{\circledR}$ VILO'M Master Mix (ThermoFisher, ON).

\subsubsection{Pharmacological treatments}

To inhibit PIKfyve, cells were pre-incubated at the indicated concentration of apilimod (Toronto Research Chemicals, Inc, ON) and period of time. Ionomycin-treatments were 
typically done for $5 \mathrm{~min}$ at $10 \mu \mathrm{M}$ in the presence of extracellular $\mathrm{Ca}^{2+}$. Alternatively, cells were pre-incubated for 30 min before phagocytosis with either $10 \mu \mathrm{M}$ BAPTA-AM, $1 \mathrm{mM}$ EGTA or $5 \mu \mathrm{M}$ xestospongin C. All drugs were maintained through the entire experiment including pulse and chase periods when performing phagocytosis and phagosome maturation assays.

\subsubsection{Cell lysates, SDS-PAGE and Western blotting}

Cells were scraped and collected after $30 \mathrm{~h}$ post electroporation of scrambled and TRPML1 siRNA and lysed using $2 X$ Laemmli sample buffer, passing through a 27 gauge needle and heated for $15 \mathrm{~min}$ at $65^{\circ} \mathrm{C}$. After $5 \mathrm{~min}$ centrifugation at $13,000 \mathrm{~g}$, the samples were loaded into a $12 \%$ SDS-polyacrylamide gels and transferred onto a PVDF membrane, followed by blocking and incubation with primary and secondary antibodies. Protein levels were visualized by enhanced chemiluminescence and BioRad gel documentation system.

\subsubsection{Phagocytosis and phagosome maturation assays}

Latex polymer beads with a mean diameter of $2.6 \mu \mathrm{m}$ or $3.9 \mu \mathrm{m}$ (Bangs Laboratories, IN) were opsonized with $10 \mathrm{mg} / \mathrm{ml}$ human IgG in PBS at room temperature for $1-2 \mathrm{~h}$ gyrating, followed by 3 washes in PBS. Phagocytosis was initiated by adding beads to cells and incubating at $37^{\circ} \mathrm{C}$ for $15 \mathrm{~min}$. Unbound beads were then washed, cells were fixed with 4\% PFA for 20 min, quenched with $100 \mathrm{mM}$ glycine for $20 \mathrm{~min}$, and stained with fluorescently labelled goat anti-human antibodies at 1:1000 dilution for 20 min to discriminate internalized beads from externally bound beads. The number of 
phagosomes per 100 cells was scored to determine the phagocytic index. Alternatively, after phagocytosis, cells were incubated at $37{ }^{\circ} \mathrm{C}$ for a chase period to allow for phagosome maturation. See below for live-cell imaging of phagocytosis.

\subsubsection{Phagosome isolation and staining for LAMP1}

Phagosome isolation and staining for LAMP1 was done as described in (G. H. E. Kim et al., 2014). Briefly, siRNA-treated RAW macrophages were grown to near confluence in T-25 culture flasks. IgG-opsonized beads were then added and incubated for $90 \mathrm{~min}$ at $37^{\circ} \mathrm{C}$ and $5 \% \mathrm{CO}_{2}$, followed by three washes with ice-cold PBS to remove unbound beads and scraped in $15 \mathrm{ml}$ of homogenization buffer $(20 \mathrm{mM}$ Tris $\mathrm{pH} 7.4,2.5 \mu \mathrm{L} / \mathrm{ml}$ protease inhibitor cocktail (Sigma), $1 \mathrm{mM} \mathrm{AEBSF}, 1 \mathrm{mM} \mathrm{MgCl}, 1 \mathrm{mM} \mathrm{CaCl}, 1 \mu \mathrm{g} / \mathrm{ml}$ RNase and 1 $\mu \mathrm{g} / \mathrm{ml}$ DNase). A cell pellet was collected by centrifugation and re-suspended in $2 \mathrm{~mL}$ of homogenization buffer and passed through a syringe with 22-gauge needle (20-25 times) to lyse the cells. A lysate pellet was then obtained and re-suspended in $200 \mu \mathrm{L}$ of PBS and overlaid onto a sucrose gradient. Samples were then centrifuged at 50,000g for

$10 \mathrm{~min}$ at $4^{\circ} \mathrm{C}$, the layer of beads were withdrawn from the sucrose gradient using a syringe with 22-gauge needle and transferred into a new tube. Phagosomes were then washed with cold PBS, fixed with 4\% PFA for 20 min and followed by $100 \mathrm{mM}$ glycine quench. After centrifugation, phagosomes were permeabilized with iced-cold methanol for 3-5 $\min$ and washed $3 \mathrm{X}$ with $0.5 \%$ bovine serum albumin to stain with anti-LAMP1 antibodies. Phagosomes were then processed as described in "Immunofluorescence" and analysed by microscopy and/or flow cytometry. 


\subsubsection{Bactericidal Colony Assay}

RAW macrophages were untreated or exposed to $10 \mathrm{nM}$ apilimod for one hour prior to addition of bacteria - apilimod was replaced with each media change to ensure continuous PIKfyve inhibition. Alternatively, RAW macrophages were electroporated with siRNA ${ }^{\text {SC }}$ or siRNA ${ }^{\text {TRP }}$, plated for $29 \mathrm{~h}$ before being fed bacteria. In all treatments, approximately $2 \times 10^{6}$ RAW macrophages were plated. Overnight E. coli DH5 $\checkmark$ cultures were sub-cultured for $1 \mathrm{~h}$ to an $\mathrm{OD}_{600}$ of 0.6 , after which bacteria were pelleted and added to RAW macrophages at a final suspension of $1 \mathrm{OD} / \mathrm{mL}$. Bacteria were then incubated with macrophages for $1 \mathrm{~h}$ to allow for phagocytosis, after which media was aspirated, macrophages were washed with PBS and replaced with DMEM media containing $100 \mu \mathrm{g} / \mathrm{mL}$ gentamicin to kill extracellular bacteria. After $30 \mathrm{~min}$, cells were either lysed immediately $(0 \mathrm{~h})$ or the media was aspirated and replaced with fresh DMEM supplemented with $10 \%$ fetal bovine serum, and incubated for $2 \mathrm{~h}$ at $37^{\circ} \mathrm{C}$ to allow time for phagosome maturation and bacteria killing. Subsequently, media was aspirated and macrophages were scraped and lysed in $200 \mu \mathrm{L}$ of $1 \%$ Triton X-100 for 5 minutes to release bacteria. Cell lysates were re-suspended in $800 \mu \mathrm{L}$ of LB broth and subjected to serial dilution. Ten microliters of each dilution were plated onto individual LB plates and incubated overnight at $37^{\circ} \mathrm{C}$. The number of colonies were then counted and recorded.

\subsubsection{Immunofluorescence}

Cells were fixed with 4\% PFA for 20 min and quenched with $100 \mathrm{mM}$ glycine for $20 \mathrm{~min}$. When necessary, external beads were then stained with anti-human IgG and then cells 
were permeabilized with either $0.5 \%$ Triton $\mathrm{X}-100$ for 10 min to stain for EEA1, or treated with ice-cold methanol for $5 \mathrm{~min}$ to stain or co-stain with anti-LAMP1 antibodies. After washing with $0.5 \%$ bovine serum albumin in PBS, cells were sequentially incubated for $1 \mathrm{~h}$ with primary and secondary antibodies at room temperature. After mounting, cells were then visualized by fluorescence confocal microscopy. LAMP1 compartments with a diameter $\geq 1 \mu \mathrm{m}$ were scored per cell per condition to quantify the extent of swelling.

\subsubsection{Microscopy, image processing and flow cytometry}

Cells were visualized with a Quorum Spinning Disk Confocal Microscope as described in (G. H. E. Kim et al., 2014). For live-cell imaging, cells on coverslips were placed on a Leiden chamber and incubated with Hepes-buffered RPMI medium at 37 ㅇ․ To follow 2FYVE-GFP dynamics, time series composed of non-saturated 8-bit grayscale frames were acquired at two frames per min. Images where then analysed by ImageJ by identifying regions of interest on the phagosomal membrane, cytosol and extracellular space (for background subtraction). Fluorescence intensity values were then extracted, followed by background subtraction and the ratio of phagosome to cytosol signal was calculated. We synchronized the dynamics of 2FYVE-GFP to the time of phagosome closure to ensure that the kinetics of phagosomal 2FYVE-GFP was not affected by possible changes in the rate of phagocytosis.

Images were processed with Adobe Photoshop (v. 7.0.1, Adobe Systems Inc.) and analyzed using ImageJ (v. 1.47 bundled with 64-bit Java). All image processing was done without altering relative values within each image and when comparing images. 
Pseudo-colour processing was done with ImageJ by enabling "Fire" in Image/Look up Tables such that white-yellow corresponded to grayscale intensities of 255-180, orangered corresponded to grayscale intensities of $180-80$ and blue-purple corresponded to grayscale intensities of 80-1.

We also used a BD FACS Calibur flow cytometer to quantify anti-LAMP1 phagosome staining. After defining the phagosome population by using beads alone, we counted 10,000 events for each sample. We then used phagosomes incubated with secondary-only (no primary) to gate for background staining. We then counted the

number of phagosomes that were above the $95^{\text {th }}$ percentile of background. The average fluorescence intensity was calculated for this population of phagosomes.

\subsubsection{Calcium imaging during phagosome maturation}

Lysosomes were labeled by allowing the cells to endocytose $2 \mu \mathrm{M}$ Alexa647-conjugated dextran (Life Technologies, ON) at $37 \stackrel{\circ}{\circ}$ for $15 \mathrm{~min}$ and then chased for $1 \mathrm{~h}$. Following, $8 \mu \mathrm{M}$ of Fluo-4AM (Life Technologies, ON) was added to the media for $45 \mathrm{~min}$, washed and chased for $45 \mathrm{~min}$ at $37^{\circ} \mathrm{C}$. Cells were then imaged live by spinning disk confocal microscopy in Hepes-buffered RPMI medium at $37^{\circ}$ C. Images were acquired before and after adding IgG coated latex polymer beads to cells. The mean intensity of cytosolic and lysosomal Fluo-4AM was measured using ImageJ by defining random regions of interest. 


\subsubsection{Statistical Analyses}

All data was subject to statistical analysis using either ANOVA, unpaired student $t$-test or paired Student's t-test as appropriate. Experiments were repeated a minimum of three times. Specific sample size is indicated in each figure legend, along with the determined mean, standard deviation and p-values.

\subsection{Acknowledgements}

The research described here was funded by a Discovery Grant from the Natural Sciences and Engineering Research Agency of Canada, by the Canada Research Chair program and with support from Ryerson University to R.J.B. R.M.D was supported by a scholarship from the Canadian Institutes of Health Research. The 1D4B anti-LAMP1 monoclonal antibody was obtained from the Developmental Studies Hybridoma Bank developed under the auspices of the NICHD and maintained by The University of lowa, Department of Biology, lowa City, IA 52242. The mCherry-tagged wild-type human TRPML1 plasmid was generously provided by Dr. Paul Luzio. The authors have no conflict of interest to declare. 


\subsection{References}

1. Flannagan RS, Jaumouillé V, Grinstein S. The cell biology of phagocytosis. Annu Rev Pathol 2012;7:61-98.

2. Underhill DM, Ozinsky A. Phagocytosis of microbes: complexity in action. Annu Rev Immunol 2002;20:825-52.

3. Vieira O V, Botelho RJ, Grinstein S. Phagosome maturation: aging gracefully. Biochem J 2002;366:689-704.

4. Fairn GD, Grinstein S. How nascent phagosomes mature to become phagolysosomes. Trends Immunol 2012;33:397-405.

5. Kielian MC, Cohn ZA. Phagosome-lysosome fusion. Characterization of intracellular membrane fusion in mouse macrophages. J Cell Biol 1980;85:754-65.

6. Pesanti EL, Axline SG. Phagolysosome formation in normal and colchicine-treated macrophages. J Exp Med 1975;142:903-13.

7. Balla T. Phosphoinositides: tiny lipids with giant impact on cell regulation. Physiol Rev 2013;93:1019-137.

8. Vieira O V, Botelho RJ, Rameh L, Brachmann SM, Matsuo T, Davidson HW, Schreiber A, Backer JM, Cantley LC, Grinstein S. Distinct roles of class I and class III phosphatidylinositol 3-kinases in phagosome formation and maturation. J Cell Biol 2001;155:19-25.

9. Kim GHE, Dayam RM, Prashar A, Terebiznik M, Botelho RJ. PIKfyve inhibition interferes with phagosome and endosome maturation in macrophages. Traffic 2014;15:1143-63.

10. Fratti RA, Backer JM, Gruenberg J, Corvera S, Deretic V. Role of phosphatidylinositol 3kinase and Rab5 effectors in phagosomal biogenesis and mycobacterial phagosome maturation arrest. J Cell Biol 2001;154:631-44.

11. Ellson CD, Anderson KE, Morgan G, Chilvers ER, Lipp P, Stephens LR, Hawkins PT. Phosphatidylinositol 3-phosphate is generated in phagosomal membranes. Curr Biol 2001;11:1631-5.

12. Birkeland HCG, Stenmark H. Protein targeting to endosomes and phagosomes via FYVE and PX domains. Curr Top Microbiol Immunol 2004;282:89-115. 
13. Gillooly DJ, Morrow IC, Lindsay M, Gould R, Bryant NJ, Gaullier JM, Parton RG, Stenmark $\mathrm{H}$. Localization of phosphatidylinositol 3-phosphate in yeast and mammalian cells. EMBO J 2000;19:4577-88.

14. Xu Y, Hortsman $\mathrm{H}$, Seet $\mathrm{L}$, Wong $\mathrm{SH}$, Hong W. SNX3 regulates endosomal function through its PX-domain-mediated interaction with PtdIns(3)P. Nat Cell Biol 2001;3:65866.

15. Urbé S, Mills IG, Stenmark H, Kitamura N, Clague MJ. Endosomal localization and receptor dynamics determine tyrosine phosphorylation of hepatocyte growth factorregulated tyrosine kinase substrate. Mol Cell Biol 2000;20:7685-92.

16. Ellson CD, Gobert-Gosse $S$, Anderson KE, Davidson K, Erdjument-Bromage $H$, Tempst $P$, Thuring JW, Cooper MA, Lim ZY, Holmes AB, Gaffney PR, Coadwell J, Chilvers ER, Hawkins PT, Stephens LR. PtdIns(3)P regulates the neutrophil oxidase complex by binding to the PX domain of p40(phox). Nat Cell Biol 2001;3:679-82.

17. Hazeki K, Nigorikawa K, Takaba Y, Segawa T, Nukuda A, Masuda A, Ishikawa Y, Kubota K, Takasuga S, Hazeki O. Essential roles of PIKfyve and PTEN on phagosomal phosphatidylinositol 3-phosphate dynamics. FEBS Lett 2012;586:4010-5.

18. Ikonomov OC, Sbrissa D, Shisheva A. Mammalian cell morphology and endocytic membrane homeostasis require enzymatically active phosphoinositide 5-kinase PIKfyve. J Biol Chem 2001;276:26141-7.

19. Ikonomov OC, Sbrissa D, Foti M, Carpentier J-L, Shisheva A. PIKfyve controls fluid phase endocytosis but not recycling/degradation of endocytosed receptors or sorting of procathepsin D by regulating multivesicular body morphogenesis. Mol Biol Cell 2003;14:4581-91.

20. De Lartigue J, Polson H, Feldman M, Shokat K, Tooze SA, Urbé S, Clague MJ. PIKfyve regulation of endosome-linked pathways. Traffic 2009;10:883-93.

21. Jefferies HBJ, Cooke FT, Jat P, Boucheron C, Koizumi T, Hayakawa M, Kaizawa H, Ohishi T, Workman P, Waterfield MD, Parker PJ. A selective PIKfyve inhibitor blocks PtdIns $(3,5) P(2)$ production and disrupts endomembrane transport and retroviral budding. EMBO Rep 2008;9:164-70.

22. Ho CY, Alghamdi TA, Botelho RJ. Phosphatidylinositol-3,5-bisphosphate: no longer the poor PIP2. Traffic 2012;13:1-8.

23. McCartney AJ, Zhang Y, Weisman LS. Phosphatidylinositol 3,5-bisphosphate: low abundance, high significance. Bioessays 2014;36:52-64. 
24. Nicot A-S, Fares H, Payrastre B, Chisholm AD, Labouesse $M$, Laporte J. The phosphoinositide kinase PIKfyve/Fab1p regulates terminal lysosome maturation in Caenorhabditis elegans. Mol Biol Cell 2006;17:3062-3074.

25. Rutherford AC, Traer C, Wassmer T, Pattni K, Bujny M V, Carlton JG, Stenmark H, Cullen PJ. The mammalian phosphatidylinositol 3-phosphate 5-kinase (PIKfyve) regulates endosome-to-TGN retrograde transport. J Cell Sci 2006;119:3944-57.

26. Martin S, Harper CB, May LM, Coulson EJ, Meunier FA, Osborne SL. Inhibition of PIKfyve by YM-201636 dysregulates autophagy and leads to apoptosis-independent neuronal cell death. PLoS One 2013;8:e60152.

27. Dong X, Shen D, Wang X, Dawson T, Li X, Zhang Q, Cheng X, Zhang Y, Weisman LS, Delling $\mathrm{M}, \mathrm{Xu} \mathrm{H}$. PI(3,5)P(2) controls membrane trafficking by direct activation of mucolipin $\mathrm{Ca}(2+)$ release channels in the endolysosome. Nat Commun 2010;1:38.

28. Munoz C, Almilaji A, Setiawan I, Föller M, Lang F. Up-regulation of the inwardly rectifying $\mathrm{K}^{+}$channel Kir2.1 (KCNJ2) by protein kinase B (PKB/Akt) and PIKfyve. J Membr Biol 2013;246:189-97.

29. Pakladok T, Almilaji A, Munoz C, Alesutan I, Lang F. PIKfyve sensitivity of hERG channels. Cell Physiol Biochem 2013;31:785-94.

30. Tsuruta F, Green EM, Rousset M, Dolmetsch RE. PIKfyve regulates CaV1.2 degradation and prevents excitotoxic cell death. J Cell Biol 2009;187:279-94.

31. Wang W, Zhang X, Gao Q, Xu H. TRPML1: an ion channel in the lysosome. Handb Exp Pharmacol 2014;222:631-45.

32. Zeevi DA, Frumkin A, Bach G. TRPML and lysosomal function. Biochim Biophys Acta 2007;1772:851-8.

33. Dong X, Wang X, Shen D, Chen S, Liu M, Wang Y, Mills E, Cheng X, Delling $M, X u H$. Activating mutations of the TRPML1 channel revealed by proline-scanning mutagenesis. J Biol Chem 2009;284:32040-52.

34. Samie M, Wang X, Zhang X, Goschka A, Li X, Cheng X, Gregg E, Azar M, Zhuo Y, Garrity AG, Gao $Q$, Slaugenhaupt S, Pickel J, Zolov SN, Weisman LS, Lenk GM, Titus S, BryantGenevier M, Southall N, Juan M, Ferrer M, Xu H. A TRP channel in the lysosome regulates large particle phagocytosis via focal exocytosis. Dev Cell 2013;26:511-24.

35. Cheng X, Zhang X, Gao Q, Ali Samie M, Azar M, Tsang WL, Dong L, Sahoo N, Li X, Zhuo Y, Garrity AG, Wang X, Ferrer M, Dowling J, Xu L, Han R, Xu H. The intracellular Ca(2+) 
channel MCOLN1 is required for sarcolemma repair to prevent muscular dystrophy. Nat Med 2014;20:1187-92.

36. Pryor PR, Reimann F, Gribble FM, Luzio JP. Mucolipin-1 is a lysosomal membrane protein required for intracellular lactosylceramide traffic. Traffic 2006;7:1388-98.

37. Thompson EG, Schaheen L, Dang H, Fares H. Lysosomal trafficking functions of mucolipin1 in murine macrophages. BMC Cell Biol 2007;8:54.

38. Shen D, Wang X, Li X, Zhang X, Yao Z, Dibble S, Dong X, Yu T, Lieberman AP, Showalter $\mathrm{HD}, \mathrm{Xu} \mathrm{H}$. Lipid storage disorders block lysosomal trafficking by inhibiting a TRP channel and lysosomal calcium release. Nat Commun 2012;3:731.

39. Pryor PR, Mullock BM, Bright NA, Gray SR, Luzio JP. The role of intraorganellar $\mathrm{Ca}(2+)$ in late endosome-lysosome heterotypic fusion and in the reformation of lysosomes from hybrid organelles. J Cell Biol 2000;149:1053-62.

40. Kiselyov KK, Ahuja M, Rybalchenko V, Patel S, Muallem S. The intracellular $\mathrm{Ca}^{2+}$ channels of membrane traffic. Channels (Austin) 6:344-51.

41. Reddy A, Caler E V, Andrews NW. Plasma membrane repair is mediated by $\mathrm{Ca}(2+)-$ regulated exocytosis of lysosomes. Cell 2001;106:157-69.

42. Gerasimenko J V, Gerasimenko O V, Petersen $\mathrm{OH}$. Membrane repair: $\mathrm{Ca}(2+)$-elicited lysosomal exocytosis. Curr Biol 2001;11:R971-4.

43. Li X, Wang $X$, Zhang $X$, Zhao $M$, Tsang WL, Zhang $Y$, Yau RGW, Weisman LS, Xu H. Genetically encoded fluorescent probe to visualize intracellular phosphatidylinositol 3,5bisphosphate localization and dynamics. Proc Natl Acad Sci U S A 2013;110:21165-70.

44. Bajno L, Peng XR, Schreiber AD, Moore HP, Trimble WS, Grinstein S. Focal exocytosis of VAMP3-containing vesicles at sites of phagosome formation. J Cell Biol 2000;149:697706.

45. Czibener C, Sherer NM, Becker SM, Pypaert M, Hui E, Chapman ER, Mothes W, Andrews NW. Ca2+ and synaptotagmin VII-dependent delivery of lysosomal membrane to nascent phagosomes. J Cell Biol 2006;174:997-1007.

46. Braun V, Fraisier V, Raposo G, Hurbain I, Sibarita J-B, Chavrier P, Galli T, Niedergang F. TIVAMP/VAMP7 is required for optimal phagocytosis of opsonised particles in macrophages. EMBO J 2004;23:4166-76.

47. Scott CC, Botelho RJ, Grinstein S. Phagosome maturation: A few bugs in the system. J. Membr. Biol. 2003;193:137-152. 
48. Vergne I, Chua J, Deretic V. Tuberculosis toxin blocking phagosome maturation inhibits a novel Ca2+/calmodulin-PI3K hVPS34 cascade. J Exp Med 2003;198:653-9.

49. Vergne I, Fratti RA, Hill PJ, Chua J, Belisle J, Deretic V. Mycobacterium tuberculosis phagosome maturation arrest: mycobacterial phosphatidylinositol analog phosphatidylinositol mannoside stimulates early endosomal fusion. Mol Biol Cell 2004;15:751-60.

50. Malik ZA, Denning GM, Kusner DJ. Inhibition of $\mathrm{Ca}(2+)$ signaling by Mycobacterium tuberculosis is associated with reduced phagosome-lysosome fusion and increased survival within human macrophages. J Exp Med 2000;191:287-302.

51. Jayachandran R, Sundaramurthy V, Combaluzier B, Mueller P, Korf H, Huygen K, Miyazaki $\mathrm{T}$, Albrecht I, Massner J, Pieters J. Survival of mycobacteria in macrophages is mediated by coronin 1-dependent activation of calcineurin. Cell 2007;130:37-50.

52. Malik ZA, Thompson CR, Hashimi S, Porter B, lyer SS, Kusner DJ. Cutting edge: Mycobacterium tuberculosis blocks $\mathrm{Ca} 2+$ signaling and phagosome maturation in human macrophages via specific inhibition of sphingosine kinase. J Immunol 2003;170:2811-5.

53. Burgoyne RD, Clague MJ. Calcium and calmodulin in membrane fusion. Biochim Biophys Acta 2003;1641:137-43.

54. Hay JC. Calcium: a fundamental regulator of intracellular membrane fusion? EMBO Rep 2007;8:236-40.

55. Attout T, Floto A, Launay P. Calcium channels in Fc receptor signaling. Curr Top Microbiol Immunol 2014;382:95-110. 


\subsection{APPENDIX B}

SUPPORTING INFORMATION FOR CHAPTER 3 

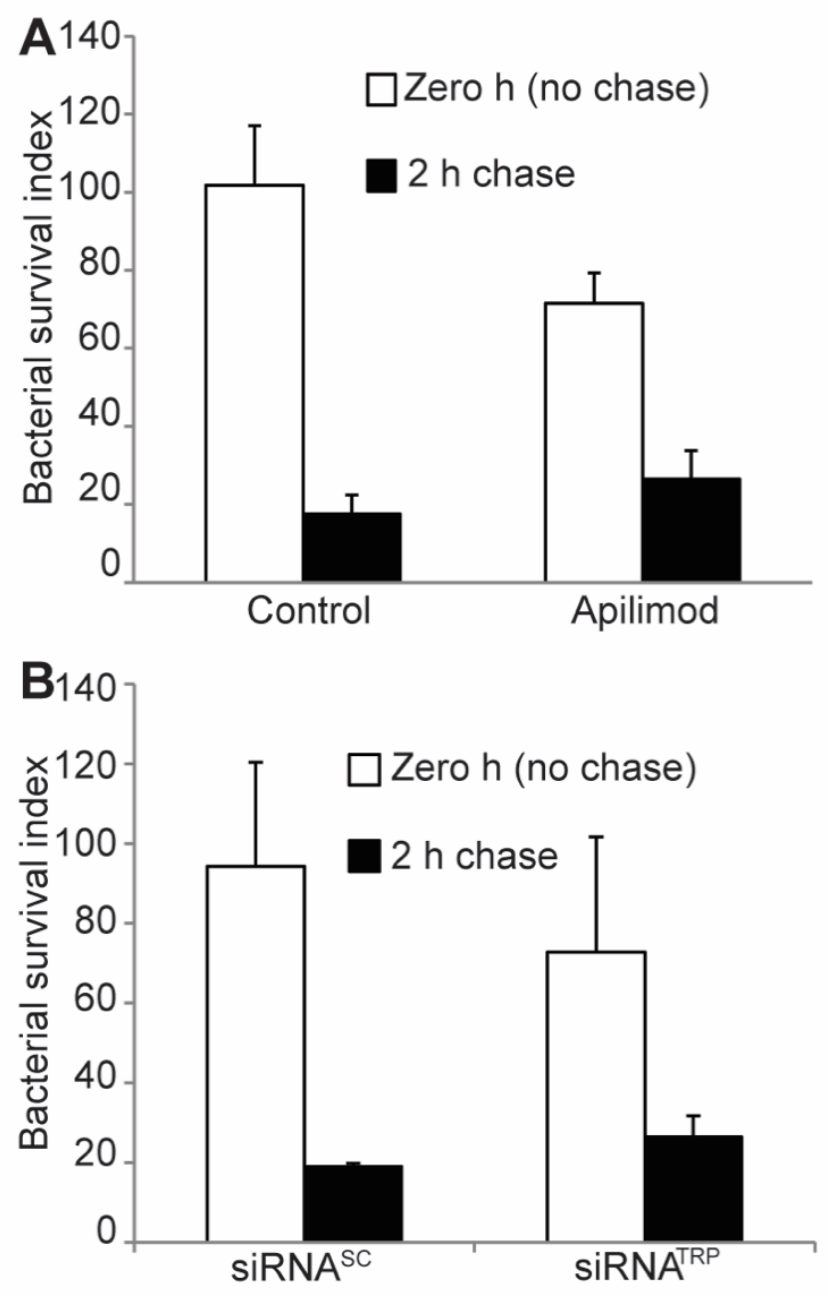

Figure S 3.1: The effect of PIKfyve and TRPML1 on bacterial uptake and survival.

A. Macrophaes were treated with DMSO or apilimod or B. electroporated with control (siRNA ${ }^{\mathrm{SC}}$ ) or TRPML1-silencing oligos (siRNA ${ }^{\text {TRP }}$ ). Macrophages were then allowed to phagocytose live $\mathrm{E}$. coli as described in Methods, followed by immediately lysis ( $0 \mathrm{~h}$, no chase) to estimate the number of internalized bacteria in each condition or chased for $2 \mathrm{~h}$ to permit bacterial killing within the phagosome. Macrophages were then lysed and lysates plated on LB-plates. The number of bacterial colonies formed were then scored. Shown is the mean \pm SEM from four independent experiments. The number of colonies formed at $2 \mathrm{~h}$ over $0 \mathrm{~h}$ estimates the rate of bacterial survival within phagosomes and is shown in Figure 4E. 


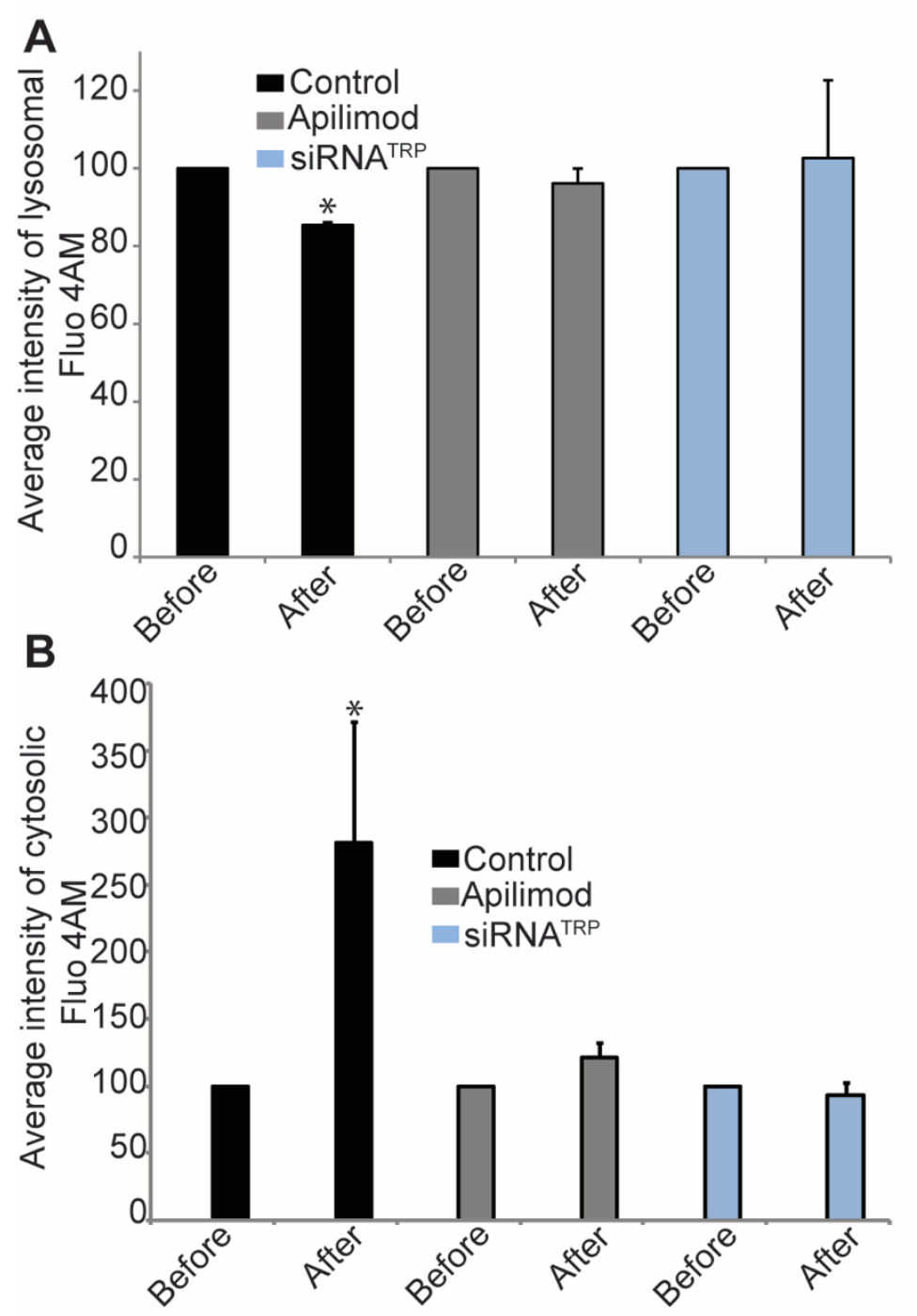

Figure S 3.2: Fluo4-AM fluorescence changes in lysosomes and cytosol of RAW macrophages.

Using the regions of interest defined in Figure 8, we measured the normalized average Fluo4AM fluorescence intensity in individual lysosomes and cytosol regions before and after phagocytosis in control, apilimod and TRPML1-silenced cells. Shown is the average \pm SEM based on at least 10 lysosome readings per cell, with least five cells per condition per experiment. Using one-way ANOVA, followed by Tukey's post-hoc test, we found that A: Fluo4-AM fluorescence in lysosomes of control cells after phagocytosis was statistically different from all other treatments $(p<0.01)$ and that B: the Fluo4-AM fluorescence in the cytosol of control cells after phagocytosis was statistically different from all other treatments $(p<0.01)$. 


\subsection{Chapter 4}

\section{The lipid kinase PIKfyve coordinates the neutrophil immune response through the activation of the Rac GTPase}

\subsection{Contributions of authors and co-authors}

Author: Roya Monica Dayam

contributions: Performed the experiments in figures 4.2, 4.3, 4.4, 4.7, and Supplementary figure4.1. Generated figures and helped in the preparation of the manuscript.

Co-Authors: Chun X. Sun, Michael Glogauer

contributions: performed the experiment in figures 4.5, and 4.6.

Co-author: Gemma Mancuso

Contributions: performed the experiment in figure 4.1 .

Co-author: Christopher H. Choy

Co-Author: Roberto Botelho

Contributions: Provided essential insight of the experiments and interpretation of the results. Wrote the manuscript.

Status of the manuscript:

Prepared for submission to a peer-reviewed journal

_ $\mathrm{X}$ _ Officially submitted to a peer-reviewed journal Accepted by a peer-reviewed journal

Published in a peer-reviewed journal

Submitted to the journal of immunology, in revisions 


\section{The lipid kinase PIKfyve coordinates the neutrophil immune}

\section{response through the activation of the Rac GTPase}

The following work has been submitted to journal of immunology

Roaya M. Dayam ${ }^{* \dagger}$, Chun X. Sun ${ }^{\ddagger}$, Gemma Mancuso $^{*}$, Christopher H. Choy ${ }^{* \dagger}$, Michael Glogauer ${ }^{\ddagger}$ and Roberto J. Botelho ${ }^{*+2}$

${ }^{*}$ Department of Chemistry and Biology and the ${ }^{\dagger}$ Molecular Science Graduate Program, Ryerson

University, Toronto, ON, Canada, M5B2K3

${ }^{\ddagger}$ Faculty of Dentistry, University of Toronto, Toronto, ON, Canada, M5S3E2 


\subsection{Abstract}

Neutrophils rapidly arrive at an infection site because of their unparalleled chemotactic ability, after which they unleash numerous attacks on pathogens through degranulation, reactive oxygen species (ROS) production, and by phagocytosis, which sequesters pathogens within phagosomes. Phagosomes then fuse with lysosomes and granules to kill the enclosed pathogens. A complex signalling network composed of kinases, GTPases and lipids such as phosphoinositides helps coordinate all these processes. There are seven species of phosphoinositides that are interconverted by lipid kinases and phosphatases. PIKfyve is a lipid kinase that generates phosphatidylinositol-3,5-bisphosphate $\left[\mathrm{PI}(3,5) \mathrm{P}_{2}\right]$, and directly or indirectly, phosphatidylinositol-5-phosphate [PtdIns(5)P]. PIKfyve inactivation causes massive lysosome swelling, disrupts membrane recycling, and in macrophages, blocks phagosome maturation. Yet, the role of PIKfyve in neutrophils has not been explored. Here, we show that PIKfyve inhibition in murine neutrophils does not affect granule morphology or degranulation, but causes LAMP1-positive lysosomes to engorge. Additionally, PIKfyve inactivation blocks phagosome-lysosome fusion in a manner that can be rescued with $\mathrm{Ca}^{2+}$ ionophores or agonists of TRPML1, a lysosomal $\mathrm{Ca}^{2+}$ channel. Strikingly, PIKfyve is necessary for chemotaxis, ROS production and stimulation of the Rac GTPases, which control chemotaxis and ROS. This is consistent with observations in non-leukocytes that showed that PIKfyve and PtdIns(5)P control Rac and cell migration. Overall, we demonstrate that PIKfyve has a robust role in neutrophils and propose a model in which PIKfyve modulates phagosome maturation through $\mathrm{PI}(3,5) \mathrm{P}_{2}$ dependent activation of TRPML1, while chemotaxis and ROS are regulated by Ptdlns(5)Pdependent activation of Rac. 
Keywords: rodent, phagocytosis, chemotaxis, lysosomes, neutrophils, lipids, innate immunity, GTPases 


\subsection{Introduction}

Neutrophils are the first responders to an infection and thus play an essential role in coordinating the innate immune response (Lee, Harrison, \& Grinstein, 2003b; Mayadas, Cullere, \& Lowell, 2014; Nauseef \& Borregaard, 2014; Nordenfelt \& Tapper, 2011). They do this because of their unmatched chemotactic ability, sensing and tracking the chemical trail to sites of infection (Mayadas et al., 2014). Once in contact with pathogens, they unleash a variety of attacks including degranulation to secrete cytokines, hydrolytic enzymes and anti-bacterial peptides, activation of the NADPH oxidase to generate reactive oxygen species (ROS), and phagocytosis, to engulf and sequester the pathogens into phagosomes (Lee et al., 2003b; Mayadas et al., 2014; Nordenfelt \& Tapper, 2011; Sheshachalam, Srivastava, Mitchell, Lacy, \& Eitzen, 2014). Phagosomes then mature by fusing with granules and lysosomes to kill and digest the pathogens (Nordenfelt \& Tapper, 2011; A. W. Segal et al., 1980).

All these responses are coordinated through a variety of receptors and intracellular signals, including small GTPases (Baker, Pan, \& Welch, 2016) and the phosphoinositide (PI) lipids (Lee et al., 2003b; Nordenfelt \& Tapper, 2011; Tuosto, Capuano, Muscolini, Santoni, \& Galandrini, 2015). Based on the phosphorylation pattern of the head group, there are seven species of PIs that typically function by differentially distributing to intracellular membranes and then recruiting a set of protein effectors specific to that PI species (Balla, 2013; Di Paolo \& De Camilli, 2006). Through this general process, phosphatidylinositol-4,5-bisphosphate and phosphatidylinositol-3,4,5-trisphosphate help to coordinate actin and membrane dynamics to direct chemotaxis and phagocytosis (Cunningham et al., 2001; Hannigan et al., 2002; Minakami et al., 2010; Sadhu, Masinovsky, Dick, Sowell, \& Staunton, 2003). In comparison, 
phosphatidylinositol-3-phosphate regulates endosomal membrane trafficking, phagosome maturation and activation of the NADPH oxidase (Anderson et al., 2010; C. D. Ellson, Anderson, et al., 2001; C. D. Ellson, Gobert-Gosse, et al., 2001; C. Ellson, Davidson, Anderson, Stephens, \& Hawkins, 2006; Minakami et al., 2010). However, there is a dearth of knowledge about the importance of phosphatidylinositol-5-phosphate [Ptdlns(5)P] and phosphatidylinositol-3,5bisphosphate $\mathrm{PI}(3,5) \mathrm{P}_{2}$ in neutrophil function.

$\mathrm{PI}(3,5) \mathrm{P}_{2}$ is synthesized by the lipid kinase PIKfyve by phosphorylating phosphatidylinositol-3-phosphate (Ho et al., 2012; Ognian C Ikonomov et al., 2002; McCartney et al., 2014). On the other hand, controversy remains about the source of Ptdlns(5)P (McCartney et al., 2014; Shisheva, Sbrissa, \& Ikonomov, 2015). In one model, PIKfyve synthesizes Ptdlns(5)P directly by phosphorylating Ptdlns (Diego Sbrissa, Ikonomov, Filios, Delvecchio, \& Shisheva, 2012; Shisheva et al., 2015), while by another view $\mathrm{PI}(3,5) \mathrm{P}_{2}$ is converted to Ptdlns(5)P via the action of the myotubularin lipid phosphatases (McCartney et al., 2014; Oppelt et al., 2013; Zolov et al., 2012). Regardless, loss of PIKfyve function causes multiple defects attesting to its importance (Ho et al., 2012), including embryonic lethality in PIKfyve $^{-/-}$mice (Ognian C. Ikonomov et al., 2011), swollen endolysosomes (O C Ikonomov et al., 2001), hindered membrane recycling (de Lartigue et al., 2009; Rutherford et al., 2006), impaired lysosomal $\mathrm{Ca}^{2+}$ signalling (Dong, Shen, Wang, Dawson, Li, Zhang, Cheng, Zhang, Weisman, Delling, \& Xu, 2010), and defective autophagic flux (Ferguson et al., 2012; Martin et al., 2013). Interestingly, PIKfyve has an emerging role in the immune response. For example, PIKfyve inhibition disrupts TLR and cytokine signalling - in fact, the PIKfyve inhibitor, apilimod, was used to suppress IL-12/IL-13 signalling before it was discovered to be a selective inhibitor of PIKfyve 
(Cai et al., 2013; Cai, Xu, Kim, Loureiro, \& Huang, 2014). In addition, mice carrying a plateletspecific PIKfyve ${ }^{-/-}$genotype suffer from massive macrophage activation and inflammation (Min et al., 2014a). Lastly, inhibition of PIKfyve blocks phagosome maturation in macrophages (G. H. E. Kim et al., 2014). This likely occurs because $\mathrm{PI}(3,5) \mathrm{P}_{2}$ is needed to activate TRPML1, a $\mathrm{PI}(3,5) \mathrm{P}_{2}$-gated lysosomal $\mathrm{Ca}^{2+}$ channel (Dayam, Saric, Shilliday, \& Botelho, 2015; Dong, Shen, Wang, Dawson, Li, Zhang, Cheng, Zhang, Weisman, Delling, \& Xu, 2010). When TRPML1 is silenced or $\mathrm{Ca}^{2+}$ is chelated, phagosomes and lysosomes dock but fail to fuse (Dayam et al., 2015). It remains possible that some of these defects are due to concomitant loss of Ptdlns(5)P when PIKfyve is inhibited. Indeed, PIKfyve and MTMR3, a myotubularin, are implicated in cell migration via the PtdIns(5)P-dependent activation of the Rac GTPase, a critical coordinator of actin remodelling (Oppelt et al., 2013, 2014).

Activation of the Rac GTPases is critically important for neutrophil chemotaxis, phagocytosis and stimulation of the NADPH oxidase (Abo et al., 1991; Knaus, Heyworth, Evans, Curnutte, \& Bokoch, 1991; Roberts et al., 1999). Given all this, we postulated that PIKfyve activity, through the synthesis of $\mathrm{PI}(3,5) \mathrm{P}_{2}$ and/or Ptdlns(5)P, is essential for coordinating various neutrophil functions. Indeed, we found that PIKfyve inhibition blocks phagosome maturation, chemotaxis and the NADPH oxidase, but not degranulation. In part, PIKfyve regulates these functions by modulating the lysosomal TRPML1 $\mathrm{Ca}^{2+}$ channel and activation of the Rac GTPases. 


\subsection{Materials and Methods}

4.4.1 Bone marrow-derived neutrophil isolation and stimulation

Bone marrow from the femur and tibia of $\mathrm{C} 57 \mathrm{BL} / 6$ mice were extracted by flushing the bones with complete DMEM (DMEM plus 10\% fetal bovine serum) using a 27-gauge needle. Cells were centrifuged at $1000 \mathrm{xg}$ for $5 \mathrm{~min}$ to yield a pellet that was then resuspended in $1 \mathrm{~mL}$ of complete DMEM and centrifuged at $1000 \mathrm{xg}$ for 30 minutes over a Percoll gradient containing $55 \%, 65 \%$, and $80 \%$ Percoll. The band of neutrophils between the $80 \%$ and $65 \%$ Percoll was collected, washed with PBS and resuspended in complete DMEM. To plate cells, glass coverslips were previously coated with $3 \%$ BSA at room temperature for 30 min, followed by a PBS wash. One million neutrophils were then plated by incubating for $30 \mathrm{~min}$ at $37{ }^{\circ} \mathrm{C}$ and at $5 \% \mathrm{CO}_{2}$. Animal handling and treatment was done according to Institutional Animal Care Ethics Board.

\subsubsection{PIKfyve inhibition and vacuolation}

To inhibit PIKfyve, neutrophils were treated with the indicated concentrations of apilimod (Cedarlane, Burlington, ON) and periods of time. To quantify vacuolation, cells were imaged live in the continuous presence of apilimod for a period of no more than 20 min using differential interference contrast microscopy. Vacuoles were defined as being greater than $1 \mu \mathrm{m}$. Alternatively, cells were stimulated and processed for live-cell imaging or fixed.

\subsubsection{Phagocytosis and phagosome maturation assays}

For phagocytosis and phagosome maturation, we used polymer beads with a diameter of 2.08 $\mu m$ (Bangs Laboratories, IN) opsonized with human IgG as described in (Dayam et al., 2015) or $\mathrm{DH} 5 \checkmark$ E. coli grown overnight and resuspend in $\mathrm{PBS}$ to a final $\mathrm{OD}_{600}$ of 1 . Particles were then 
added to neutrophils and synchronized by centrifugation of the cells for $5 \mathrm{~min}$ at $400 \mathrm{xg}$. Afterwards, cells were washed $3 x$ with PBS and incubated with complete DMEM at $37{ }^{\circ} \mathrm{C}$ and $5 \% \mathrm{CO}_{2}$ for 15 min to allow internalization of the beads or E. coli. For phagocytic index, cells were fixed with $4 \%$ PFA for 20 min at room temperature, followed by quenching with $100 \mathrm{mM}$ glycine for $20 \mathrm{~min}$. For phagosome maturation, cells were incubated for a chase time of $1 \mathrm{~h}$ at $37{ }^{\circ} \mathrm{C}$ and $5 \% \mathrm{CO}_{2}$ before fixing and processing for immunofluorescence.

\subsubsection{Stimulation of degranulation}

Neutrophils were plated and pre-treated with $30 \mathrm{nM}$ of apilimod for $30 \mathrm{~min}$ at $37{ }^{\circ} \mathrm{C}$ prior to addition of $1 \mu \mathrm{M}$ latrunculin A (Abcam, Cambridge, MA) for $30 \mathrm{~min}$. Degranulation was induced by adding $300 \mathrm{nM}$ of fMLP (Sigma-Aldrich, Oakvile, ON) for 3 min followed by fixation with $4 \%$ PFA for $20 \mathrm{~min}$ and quenching with $100 \mathrm{mM}$ glycine. To assess degranulation for primary granules cells were stained with anti-CD63 antibodies (see below).

\subsubsection{Immunofluorescence}

After the required manipulation, neutrophils were washed and fixed with $4 \%$ paraformaldehyde (PFA) for 20 min, followed by 3x PBS washes and quenching using $100 \mathrm{mM}$ glycine in PBS for 20 min. For intracellular immunostaining, cells were permeabilized with either $0.5 \%$ Triton $\mathrm{X}-100$ for $10 \mathrm{~min}$ at room temperature to stain with rabbit anti-mouse polyclonal antibodies to MPO, MMP9, lactoferrin (all used at 1:100, One World Lab, San Diego, CA), or

CD63 (H-193, 1:200, Santa Cruz Biotech, Paso Robles, CA). Alternatively, cells were permeabilized with $100 \%$ ice-cold methanol for 5 min to stain with rat anti-mouse LAMP1 monoclonal antibodies (clone 1D4B, 1:100, Developmental Hybridoma Bank, lowa City, IO). For 
extracellular staining, no permeabilization was performed before immunostaining. All primary antibodies were incubated for $1 \mathrm{~h}$ at room temperature in $0.5 \%$ BSA, followed by $3 x$ PBS washes and $1 \mathrm{~h}$ with fluorescently-labelled secondary antibodies at 1:1000 (Cedarlane, Burlington, ON). Lastly, cells were washed every 5 min with $0.5 \%$ BSA for 30 min to remove the excess secondary antibodies. The coverslips were mounted with Dako mounting media and visualized.

\subsubsection{Microscopy and image analysis}

For scoring vacuolation, live-cell imaging was done using an inverted Olympus IX83 microscope (Olympus, Richmond Hill, ON) with a Hamamatsu ORCA-flash 4.0 digital camera. Cells plated on coverslips were placed in a chamber with HEPES-buffered RPMI medium at $37{ }^{\circ} \mathrm{C}$ and were imaged by differential interference contrast (DIC). For fluorescence imaging, we used a Quorum spinning disk confocal microscope (Quorum, Guelph, ON) equipped with a Hamamatsu C910013 EMCCD camera and 100X oil objective (NA 1.4) to obtain single-plane images. Images were then analyzed using ImageJ (v. 1.47 bundled with 64-bit Java) and processed with Adobe Photoshop (v. 7.0.1, Adobe Systems Inc., San Jose, CA) without altering the relative fluorescence intensity of the images. To quantify phagosome maturation, phagosomeassociated LAMP1 fluorescent intensities were clustered into three groups (strong, intermediate and weak) using pseudo-color processing as described in (Dayam et al., 2015; G. H. E. Kim et al., 2014).

\subsubsection{Zigmond Chamber Chemotaxis Assay}

One million bone-marrow neutrophils in $100 \mu \mathrm{HBSS}$ with $1 \%$ gelatin were incubated with different concentrations of apilimod for 30 minutes at $37^{\circ} \mathrm{C}$. They were then plated onto $5 \%$ 
BSA-coated microscope cover glass $(22 \times 40 \mathrm{~mm})$ for 10 minutes. The cover glass was then inverted onto a Zigmond chamber with $100 \mu \mathrm{L}$ HBSS media and $100 \mu \mathrm{L}$ HBSS containing $1 \mu \mathrm{M}$ fMLP added to the right and left chambers, respectively. Time-lapse video microscopy was used to record neutrophil movements in the Zigmond chambers for 15 minutes (1 frame/20 seconds). Captured images were analyzed using cell-tracking software (Retrac Version 2.1.01 freeware) for cell direction and speed. Data were collected from five independent experiments.

\subsubsection{Cytochrome C Oxidation Assay}

To measure reactive oxygen species generation, we employed oxidation of cytochrome C. One million bone-marrow neutrophils in $100 \mu$ PBS with 10 mM D-glucose were incubated with different concentrations of apilimod for 30 minutes. They were then mixed with $880 \mu \mathrm{PICM}-\mathrm{G}$ (138 mM NaCl, $2.7 \mathrm{mM} \mathrm{KCL}, 0.6 \mathrm{mM} \mathrm{CaCl}$, $1.0 \mathrm{mM} \mathrm{MgCl}$, $5 \mathrm{mM}$ glucose, $10 \mathrm{mM}$ $\mathrm{NaH}_{2} \mathrm{PO}_{4} / \mathrm{Na}_{2} \mathrm{HPO}_{4}, \mathrm{pH}$ 7.4) supplemented with $0.1 \mathrm{mM}$ cytochrome $\mathrm{C}$ and incubated for another 10 minutes at $37^{\circ} \mathrm{C}$. Cells were then stimulated with $1 \mu \mathrm{M}$ fMLP or $1 \mu \mathrm{M}$ PMA for 30 minutes at $37^{\circ} \mathrm{C}$. The absorbance of reduced cytochrome $\mathrm{C}$ at $550 \mathrm{~nm}$ was then recorded and background corrected (reaction lacking cell lysates). Data were collected from five independent experiments.

\subsubsection{Preparation of recombinant GST-PBD protein}

To quantify Rac GTPase activation, we employed affinity chromatography using a fusion protein of glutathione-S-transferase (GST) and the p21-binding domain (PBD) of PAK as described previously but with a few modifications (Benard, Bohl, \& Bokoch, 1999). Briefly, recombinant

proteins were induced in $\mathrm{BL} 21^{*} \mathrm{E}$. coli in the presence of $0.4 \mathrm{mM}$ IPTG for $3 \mathrm{~h}$ at $30{ }^{\circ} \mathrm{C}$. Fifty ODs 
of bacterial culture were then centrifuged before addition of $50 \mathrm{~mL}$ of bacterial lysis buffer (10 $\mathrm{mM}$ Tris, pH 8.0, $1 \mathrm{mM}$ EDTA, $150 \mathrm{mM} \mathrm{NaCl}, 100 \mu \mathrm{g} / \mathrm{mL}$ lysozyme, $5 \mathrm{mM}$ DTT, $1 \%$ triton X-100, and supplemented with bacterial protease inhibitor cocktail (Bio Basic, Markham, ON) and 1 mM PMSF. Bacteria were then grinded using a pastel and mortar with $1 \mathrm{~g}$ of celite (SigmaAldrich). Lysates were then cleared by centrifugation and supernatant was added to reduced glutathione-sepharose (Invitrogen, Carlsbad, CA) and incubated at $4{ }^{\circ} \mathrm{C}$ for $1 \mathrm{~h}$ with agitation followed by $3 x$ wash with bacterial lysis buffer.

\subsubsection{Affinity precipitation of GTP-bound Rac GTPase and Western blotting}

One million neutrophils were pre-treated at $37{ }^{\circ} \mathrm{C}$ for $30 \mathrm{~min}$ with either $50 \mathrm{nM}$ of apilimod or DMSO, followed by addition of $1 \mu \mathrm{M}$ fMLP or vehicle for $1 \mathrm{~min}$. Cells were placed immediately on ice and lysed with $100 \mu \mathrm{L}$ of ice-cold 5X MLB lysis buffer (125 mM HEPES, pH 7.5, $25 \mathrm{mM}$ EDTA, 1\% Triton X-100, $750 \mathrm{mM} \mathrm{NaCl}, 25 \mathrm{mM} \mathrm{MgCl}_{2}$, and $50 \%$ glycerol, supplemented with mammalian protease inhibitors (Sigma-Aldrich). Cell lysates were clarified by centrifugation at $10,000 \mathrm{xg}$ for $5 \mathrm{~min}$ at $4{ }^{0} \mathrm{C}$. Ten percent of cell lysates were removed to measure protein levels across each sample. The remaining cell lysates were incubated with $50 \mu \mathrm{L}$ of glutathioneSepharose beads attached to GST-PBD or GST (50\% suspension) and incubated for $1 \mathrm{~h}$ at $4{ }^{\circ} \mathrm{C}$ with agitation. The samples were centrifuged for $2 \mathrm{~min}$ at 10,000 $\mathrm{xg}$ and the supernatant was removed. The pellets were washed $3 x$ with $1 x M L B$ lysis buffer before protein elution with $2 x$ Laemmli buffer containing 2-mercaptoethanol. Protein eluants were then loaded and separated in a $12 \%$ SDS-PAGE, transferred to a PVDF membrane and processed for Western blotting with mouse anti-Rac1 antibodies (clone 23A8, 1:2500, Genetex, Irvine, CA ) and HRP-linked goat antimouse secondary antibodies used at 1:10000 (Cedarlane). Enhanced chemiluminescence was 
detected and analysed by band densitometry using a Bio-Rad gel documentation system (BioRad, Mississauga, ON).

\subsubsection{Statistical Analyses}

All experiments were repeated at least three times and all data was subjected to statistical analysis using either unpaired or paired Student's t-test for single-parameter experiments or using ANOVA and Tukey's post-hoc test for multi-parameters experiments. Statistical significant was drawn at $p<0.05$. 


\subsection{Results}

4.5.1 Lysosomes but not granules vacuolate in PIKfyve-inhibited neutrophils

The importance of PIKfyve activity in neutrophils has not been previously examined. To investigate this, we employed a pharmacological approach to acutely block PIKfyve activity by using apilimod, a selective antagonist of PIKfyve (Cai et al., 2013). First, we examined the sensitivity of neutrophils to apilimod by testing different concentrations and incubation times and scoring the number of vacuoles $>$ than $1 \mu \mathrm{m}$ in diameter. By incubating cells for $1 \mathrm{~h}$, we noted a gradual rise in the number of vacuoles in neutrophils exposed to increasing amounts of apilimod (Fig. 4.1A, C). We then employed an intermediate concentration of $20 \mathrm{nM}$ to examine the rate of vacuolation. Neutrophils began to significantly vacuolate within $30 \mathrm{~min}$ of drug exposure and became highly vacuolated at $90 \mathrm{~min}$ of exposure (Fig. 4.1B, D). Thus, to minimize off-target and indirect effects of PIKfyve inhibition, we generally treated neutrophils for less than $1 \mathrm{~h}$ at $<50 \mathrm{nM}$ apilimod, unless otherwise noted. 

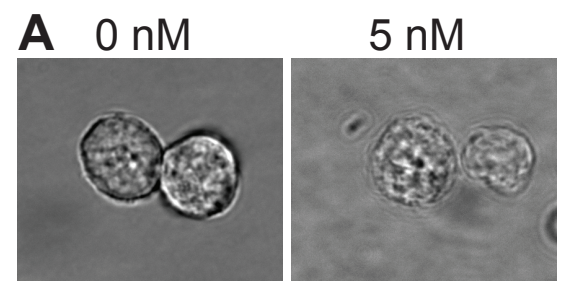

$10 \mathrm{nM}$

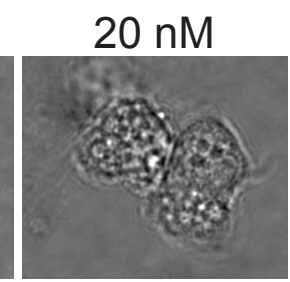

$30 \mathrm{nM}$
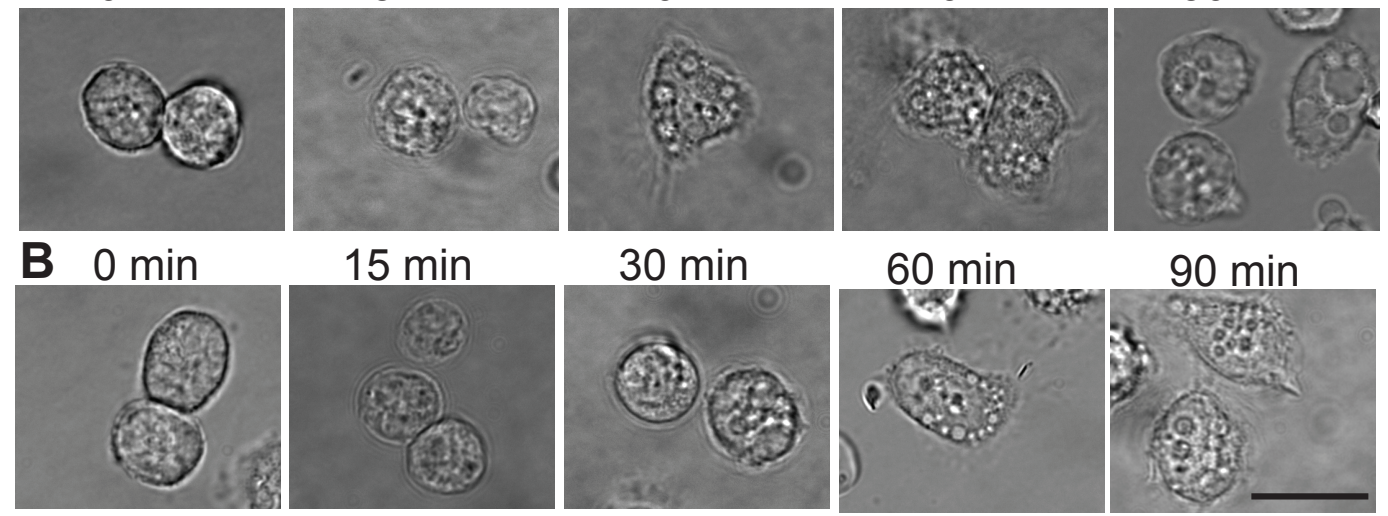

C
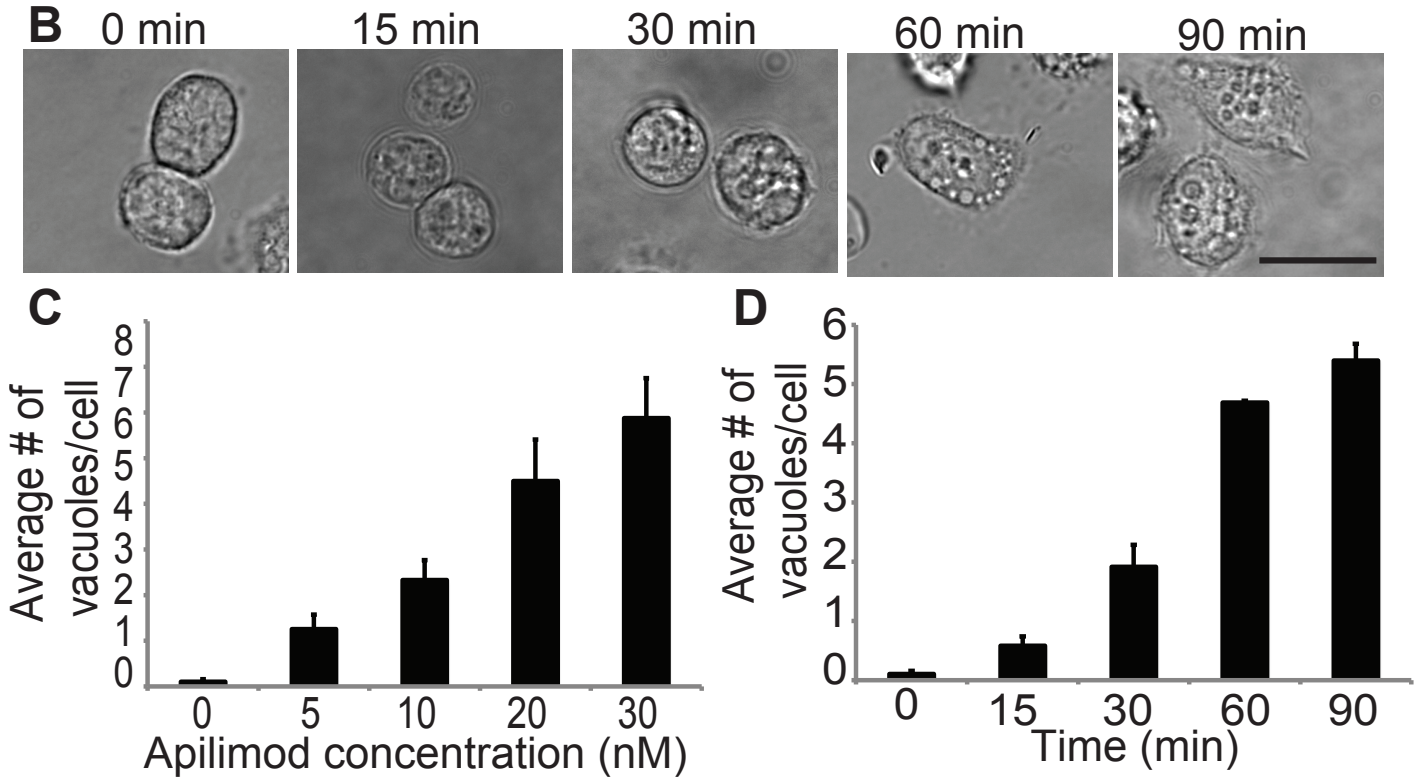

Figure 4.1: Potency and kinetics of apilimod-induced vacuolation in neutrophils.

A. Neutrophils were exposed to vehicle or to the indicated apilimod concentrations for $1 \mathrm{~h}$, followed by live-cell imaging. B. Neutrophils were exposed to vehicle or $20 \mathrm{nM}$ apilimod for the indicated time points, before live-cell imaging. C and D. Vacuolation was quantified by scoring the number of vacuoles greater than $1 \mu \mathrm{m}$ in diameter per cell. Shown are the mean number of vacuoles per neutrophil \pm SEM from $n=3$ independent experiments. Asterisk $(*)$ indicates statistically difference $(p<0.05)$ relative to control (vehicle or $t=0 \mathrm{~min}$ ) using Student's $t$-test.

We next attempted to identify the nature of the vacuoles in neutrophils. Neutrophils are not only equipped with lysosomes, but also possess the lysosome-related primary granules (or azurophilic granules), secondary and tertiary granules (Cieutat et al., 1998; Dell'Angelica, Mullins, Caplan, \& Bonifacino, 2000; Mayadas et al., 2014), which can be labelled with antibodies to LAMP1, MPO, MMP9 and lactoferrin, respectively. While we clearly observed vacuolation of LAMP1-positive lysosomes in neutrophils treated for $1 \mathrm{~h}$ with $20 \mathrm{nM}$ apilimod, 
we did not discern vacuolation of organelles that labelled with the other markers (Fig. 4.2). This suggests that LAMP1-positive lysosomes are susceptible to swelling, while primary, secondary and tertiary granules resist enlargement in neutrophils acutely inhibited for PIKfyve.

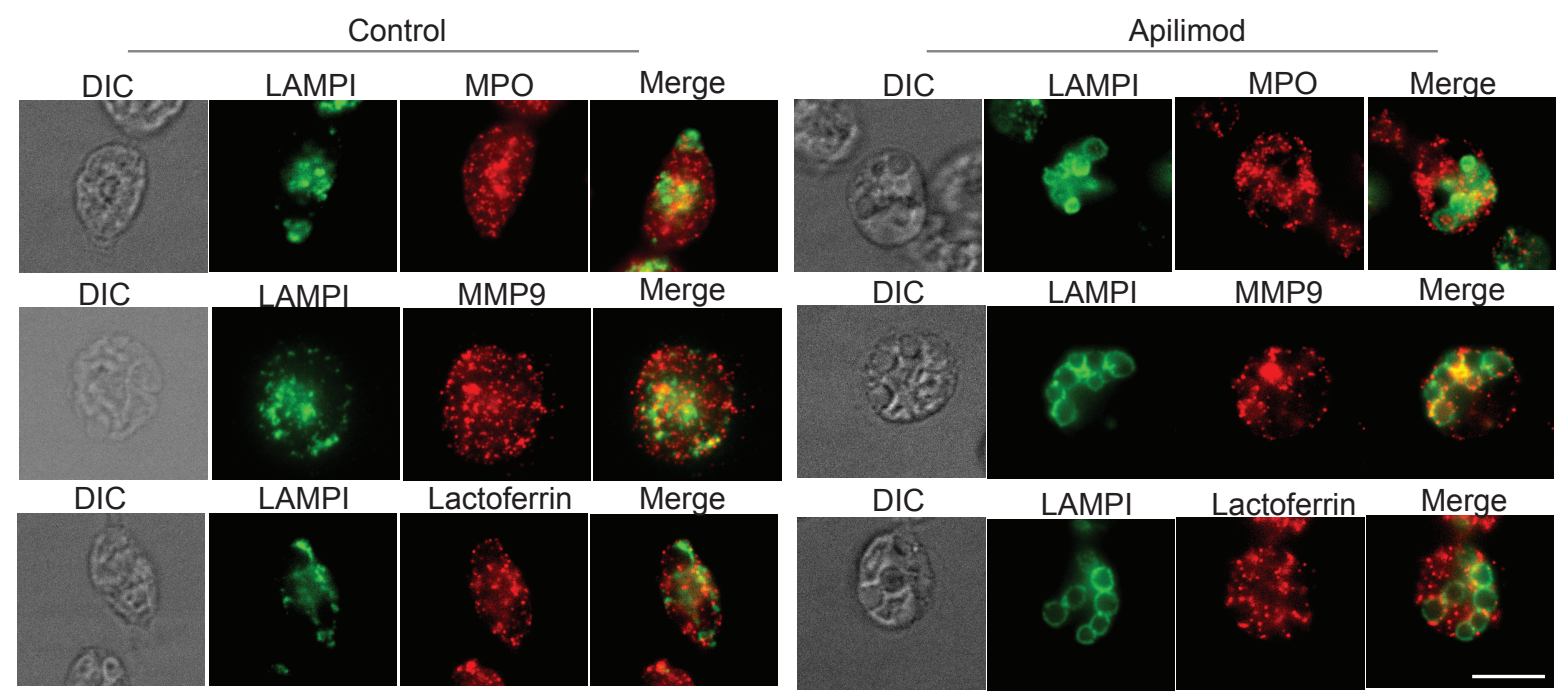

Figure 4.2: PIKfyve inhibition engorges lysosomes but not granules.

Neutrophils were treated with vehicle (control) or $20 \mathrm{nM}$ apilimod for $1 \mathrm{~h}$ before processing and staining for LAMP1, MPO, MMP9 and lactoferrin, which respectively identify lysosomes and primary, secondary and tertiary granules. Apilimod caused swelling of LAMP1-positive structures while granules remained punctate. Scale bar $=10 \mu \mathrm{m}$.

\subsubsection{PIKfyve controls phagosome-lysosome fusion in neutrophils}

We had previously shown that PIKfyve has an important role in phagosome maturation in macrophages (G. H. E. Kim et al., 2014). Since neutrophils are also professional phagocytes, we assessed the role of PIKfyve in phagocytosis and phagosome maturation. First, we evaluated the ability of neutrophils to engulf non-opsonized E.coli by measuring the phagocytic index before and after apilimod-treatment. As indicated in Figure 4.3A, the phagocytic index of neutrophils pre-treated with up to $20 \mathrm{nM}$ apilimod was similar to vector-treated neutrophils, this despite extensive vacuolation. However, treatment with $30 \mathrm{nM}$ apilimod caused a 
reduction in phagocytic appetite (Fig. 4.3A), which is reminiscent of our prior observations with macrophages (G. H. E. Kim et al., 2014).

To investigate the impact on phagosome maturation, we then treated neutrophils with $20 \mathrm{nM}$ apilimod, which is sufficient to vacuolate neutrophils but is permissive for phagocytosis. After elapsing $1 \mathrm{~h}$ post-phagocytosis to elicit phagosome maturation, we processed, stained and quantified phagosomal acquisition of LAMP1 to track phagosome-lysosome fusion as previously described (Beertsen et al., 2008; Dayam et al., 2015; G. H. E. Kim et al., 2014). We observed a remarkable inhibition of phagosome maturation in cells blocked for PIKfyve. In vector-treated neutrophils, 60\% of phagosomes labelled strongly with LAMP1 (LAMP1 ${ }^{+}$) while only $10 \%$ were negative (LAMP1; Fig. 4.3B, C). In striking comparison, neutrophils inhibited for PIKfyve had $<5 \%$ of their phagosomes as LAMP ${ }^{+}$, while $~ 70 \%$ scored as LAMP1 ${ }^{-}$(Fig. $4.3 B, C$ ). By contrast, control and apilimod-treated cells had similar levels of MPO signal associated with phagosomes, suggesting that PIKfyve activity is not necessary for phagosome fusion with primary granules (Supplemental Fig. S4.1).

We then assessed the mechanism by which PIKfyve might control phagosome maturation in neutrophils. Given our prior work in macrophages, we postulated that PIKfyve is necessary to stimulate TRPML1 to release lysosomal $\mathrm{Ca}^{2+}$ and trigger phagosome-lysosome fusion. To test this, we exposed apilimod-treated cells to ionomycin, a $\mathrm{Ca}^{2+}$ ionophore, or to MLSA1, a TRPML1 agonist to see if these agents could rescue phagosome-lysosome fusion in PIKfyve-inhibited cells. First, and as a control, we showed that ionomycin or MLSA1 alone did not impact LAMP1-staining of phagosomes (Figure 4.3B, C). Second, and most pressing, both ionomycin and MLSA1 were able to decrease the number of phagosomes devoid of LAMP1 
staining, which went from $\sim 70 \%$ in apilimod-alone neutrophils to $\sim 30 \%$ in apilimod-treated cells exposed to either ionomycin or MLSA1 (Fig. 4.3C, D). However, the rescue was partial and most phagosomes became partially-stained with LAMP1, as defined in the Methods section. Consistent with a role of TRPML1 and lysosomal $\mathrm{Ca}^{2+}$ in phagosome-lysosome fusion in neutrophils, we showed that $\mathrm{Ca}^{2+}$ chelation with BAPTA-AM potently hindered LAMP1-labelling of phagosomes (Fig. 4.3C, D). Overall, these data suggest that PIKfyve controls phagosomelysosome fusion in neutrophils in part by stimulating TRPML1 and releasing lysosomal $\mathrm{Ca}^{2+}$ to trigger fusion. This is consistent with our previous work in macrophages (Dayam et al., 2015), and previously observed peri-phagosomal increase in cytosolic $\mathrm{Ca}^{2+}$ in neutrophils (Clark \& Petty, 2010; Jaconi et al., 1990). 


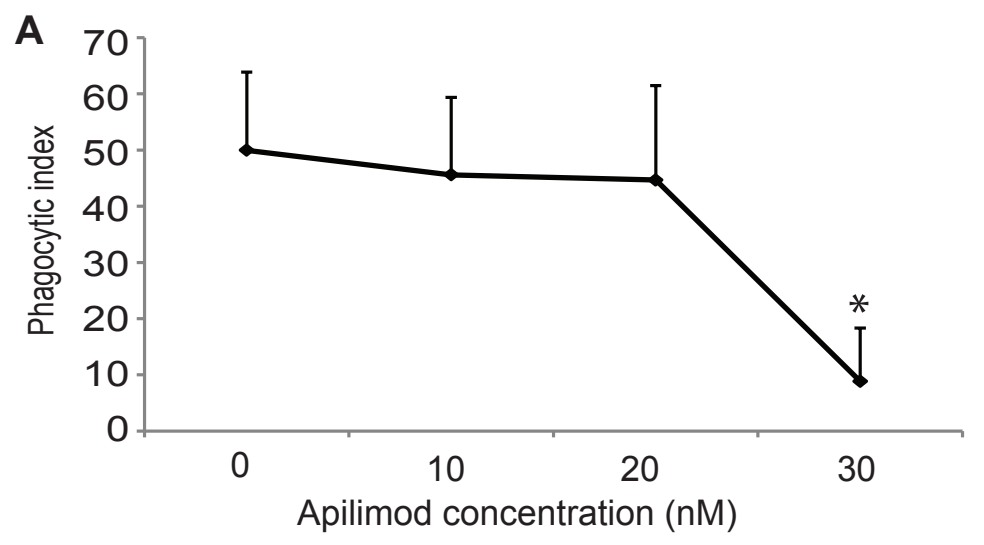

B
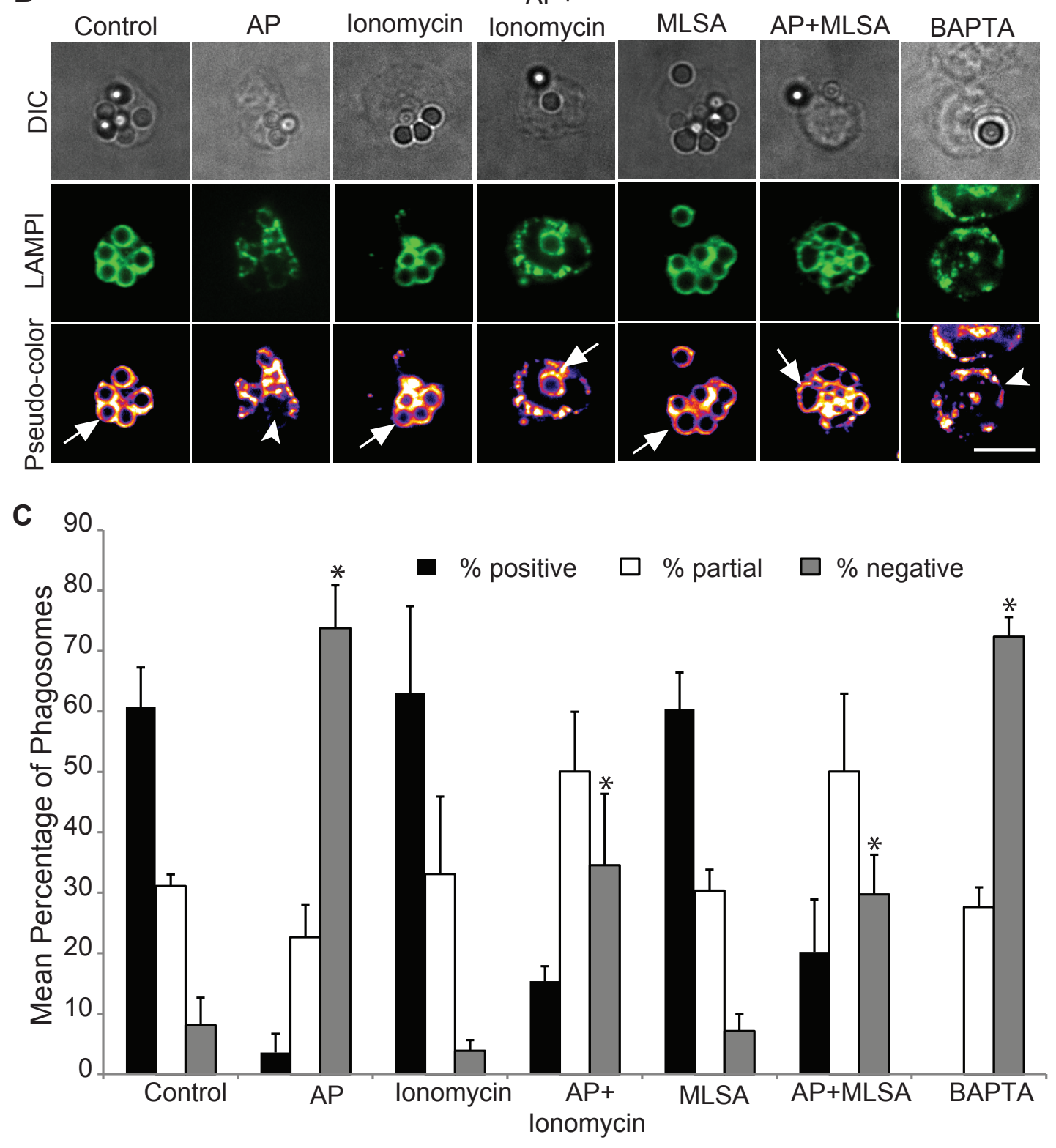
Figure 4.3: PIKfyve inhibition blocks phagosome-lysosome fusion.

A. Bimodal effect of PIKfyve inhibition on phagocytosis. Neutrophils were exposed to vehicle or with apilimod as indicated for $1 \mathrm{~h}$ before challenged with E. coli for $20 \mathrm{~min}$. The number of internalized particles was then scored as the phagocytic index (number of particles per 100 cells). B. Neutrophils were treated as control or with $20 \mathrm{nM}$ apilimod for $1 \mathrm{~h}$ before phagocytosis of IgG-coated beads for $20 \mathrm{~min}$ and maturation for $1 \mathrm{~h}$. Cells were then stained for LAMP1 to determine phagosome maturation. Please see Methods for details. Ionomycin and MLSA1 co-treatment with apilimod partially rescued LAMP1 staining of phagosomes. Arrows indicate LAMPI-positive phagosomes and arrowheads indicate LAMPI-negative phagosomes. $\mathbf{C}$. Semi-quantification of LAMP1 staining of phagosomes as described in Methods section. Apilimod inhibits labelling of phagosomes with LAMP1. This is partially rescued with ionomycin and MLSA1 treatment. A, C and D: Data are shown as the mean \pm SEM from $n=3$ independent experiments, followed by analysis with ANOVA and Tukey's post-hoc test. Asterisk indicates statistically significant difference $(p<0.05)$. Where shown, scale bar $=10 \mu \mathrm{m}$.

\subsubsection{PIKfyve is not necessary for $\mathrm{AMLP}$-induced degranulation}

In addition to phagocytosis and phagosome maturation, neutrophils rely on degranulation to eliminate pathogens (Sheshachalam et al., 2014). Since PIKfyve and TRPML1 have been linked to regulated exocytosis (Gerasimenko et al., 2001; Osborne et al., 2008; M. Samie et al., 2013), we postulated that PIKfyve activity might govern some aspects of degranulation. Given that primary granules are lysosome-related organelles and their secretion can depend on $\mathrm{Ca}^{2+}$ (Lew et al., 1986; Niessen, Kuijpers, Roos, \& Verhoeven, 1991; Sheshachalam et al., 2014; Tapper, Furuya, \& Grinstein, 2002), we examined the appearance of CD63 on the cell surface after stimulation with fMLP. We observed that resting neutrophils and those treated with only apilimod had comparable levels of surface-level CD63, showing that PIKfyve inhibition does not affect basal levels of degranulation. In comparison, fMLP exposure enhanced the levels of cell surface CD63 relative to resting neutrophils (Fig. 4.4). The fMLP-induced degranulation was unabated in neutrophils pre-treated with the PIKfyve antagonist (Fig. 4.4). Overall, these data 
show that acute loss of PIKfyve activity does not impair fMLP-induced exocytosis of primary granules, though we cannot rule out an effect during chronic PIKfyve loss.
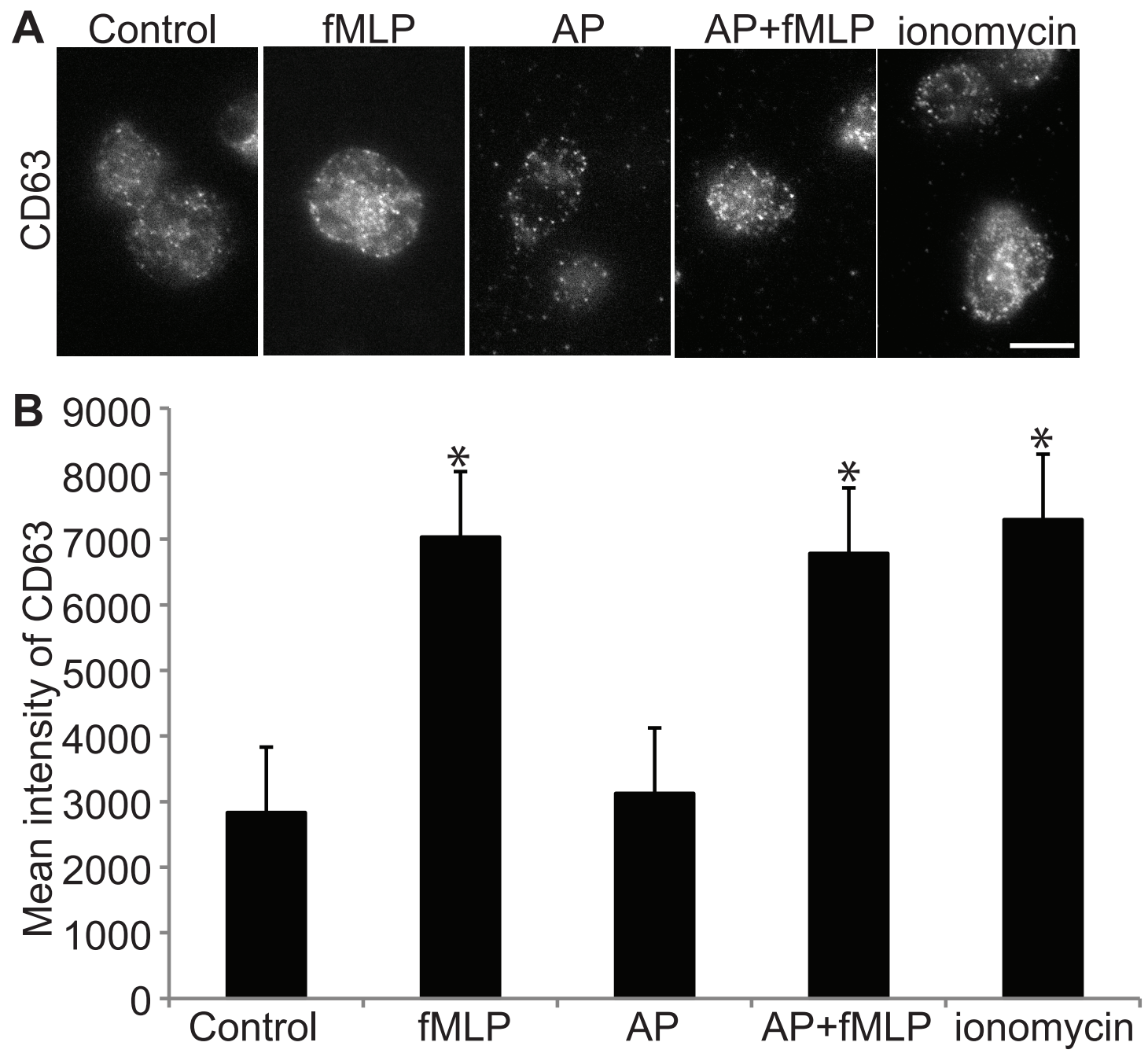

Figure 4.4: PIKfyve activity does not affect degranulation.

A. Neutrophils were treated with the indicated conditions, followed by fixation and staining of external CD63. Scale bar $=10 \mu \mathrm{m}$. B. Fluorescence intensity of external CD63 in neutrophils treated as indicated. Data are shown as the mean \pm SEM from $n=3$ independent experiments, followed by analysis with ANOVA and Tukey's post-hoc test. Asterisk indicates statistically significant difference from control $(p<0.05)$. 


\subsubsection{PIKfyve activity is necessary for fMLP-directed chemotaxis}

PIKfyve and MTMR3 cooperate to synthesize Ptdlns(5)P, which together activate the Rac GTPase to coordinate cell migration (Oppelt et al., 2013, 2014; Viaud et al., 2014). Hence, we postulated that PIKfyve activity might regulate neutrophil chemotaxis. To test this hypothesis, we examined the ability of neutrophils to move towards an FMLP chemical gradient by quantifying their speed and directionality towards the fMLP source at different concentrations of apilimod. As expected, vector-exposed neutrophils exhibited remarkable capacity to orient and move towards the fMLP gradient. By mapping the position of neutrophils relative to their starting position, we found that $\sim 80 \%$ of neutrophils had moved towards the fMLP gradient (within the right most quadrants in Figure 4.5A) and travelled at an average speed of $8.8 \pm 0.2$ $\mu \mathrm{m} / \mathrm{min}$ (Fig. 4.5B). Strikingly, at concentrations as low as $10 \mathrm{nM}$ apilimod, neutrophils became disoriented with only $54 \%$ of the cells moving towards the gradient (Fig. $4.5 \mathrm{~A}$ ). At 35 and $70 \mathrm{nM}$ apilimod, neutrophils were effectively spread equally across each quadrant and travelled a shorter overall distance (Fig. 4.5A), which effectively suggests randomized and slower movement by neutrophils. Indeed, neutrophil speed was significantly abated at $10 \mathrm{nM}$ apilimod $(5.1 \pm 0.5 \mu \mathrm{m} / \mathrm{min})$ and brought to a near standstill at $70 \mathrm{nM}$ (Fig. 4.5B). Overall, these experiments reveal an incredibly important function for PIKfyve activity in neutrophil chemotaxis. 


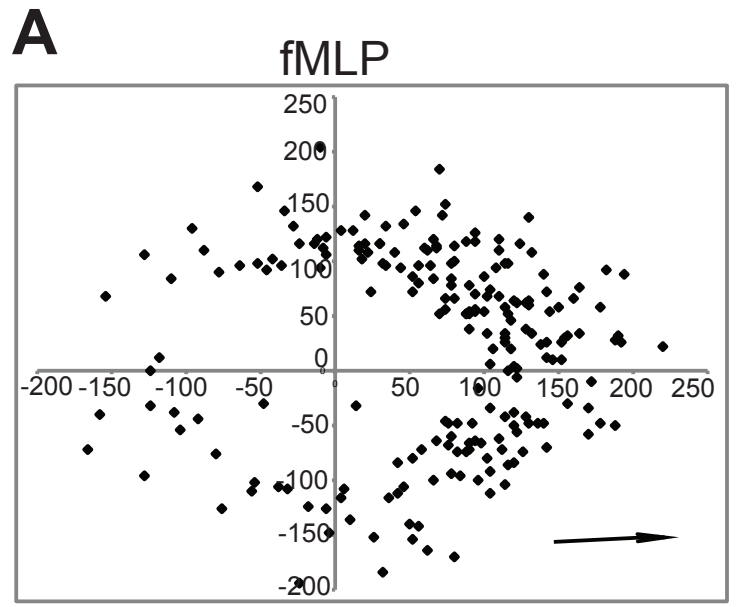

35 nM Apilimod+fMLP

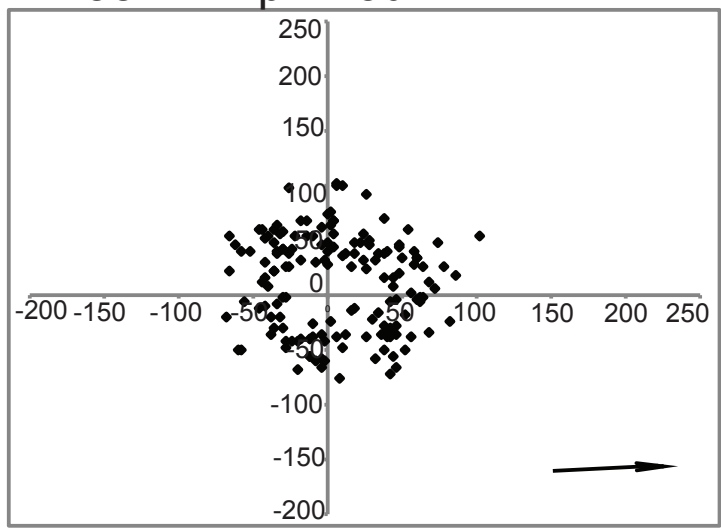

B 100

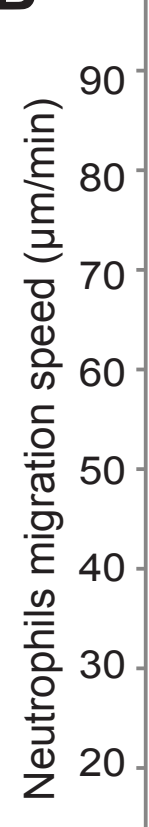

10

0
10 nM Apilimod+fMLP

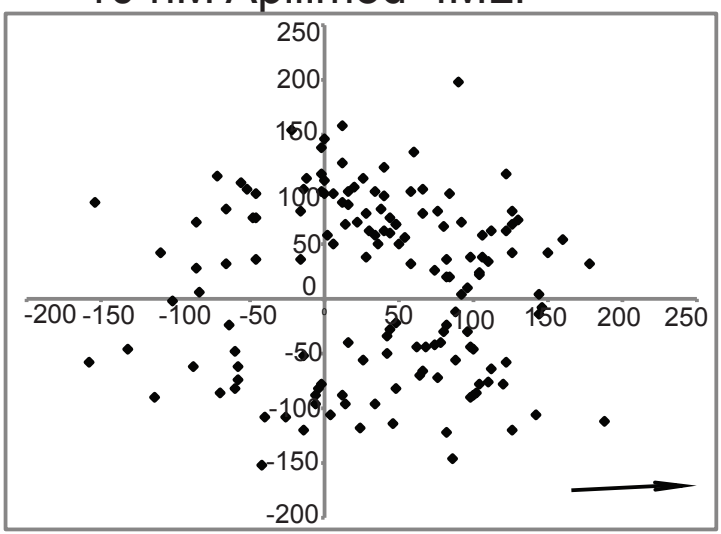

70nM Apilimod+fMLP

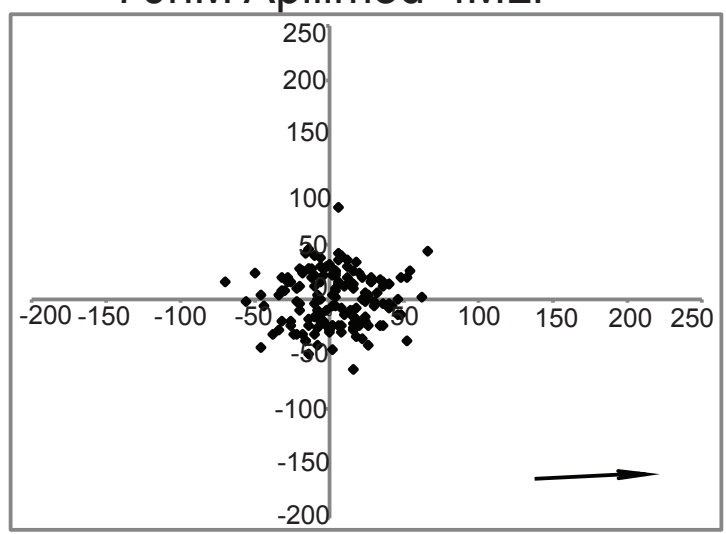


Figure 4.5: PIKfyve activity is important for neutrophil chemotaxis.

A. Neutrophils were exposed to fMLP gradient in a Zigmond chamber in the absence or presence of apilimod as indicated. Shown is the positional displacement of each cell after 15 min relative to starting point. Cells found in the right most quadrants moved towards the fMLP source. B. Neutrophil migration speed was calculated by tracking each neutrophil over $15 \mathrm{~min}$. Data are shown as the mean \pm SEM from $n=5$ independent experiments, followed by analysis with ANOVA and Tukey's post-hoc test. Asterisk indicates statistically significant difference $(p<0.05)$.

\subsubsection{PIKfyve activity is necessary for fMLP-induced ROS production}

Lastly, we considered the possibility that PIKfyve activity may be necessary for ROS synthesis, which primarily occurs through activation of the NADPH oxidase (Nauseef \& Borregaard, 2014). To test this, we measured ROS production in response to fMLP in control and apilimod-treated neutrophils using the Oxydase assay. Resting neutrophils and those treated with apilimodalone at 10, 35 or $70 \mathrm{nM}$ had similar levels of ROS production (Fig. 4.6). As expected, neutrophils exposed to stimulants like fMLP or phorbol esters displayed a large increase in ROS (Fig. 4.6). Even at $10 \mathrm{nM}$ apilimod pre-treatment, ROS production was subdued in neutrophils exposed to fMLP or PMA (Fig. 4.6). At 35 and $70 \mathrm{nM}$ apilimod, ROS synthesis was essentially thwarted in stimulant-exposed neutrophils (Fig. 4.6). Overall, this suggests that PIKfyve activity is important for ROS generation, likely by stimulating the NADPH oxidase. 


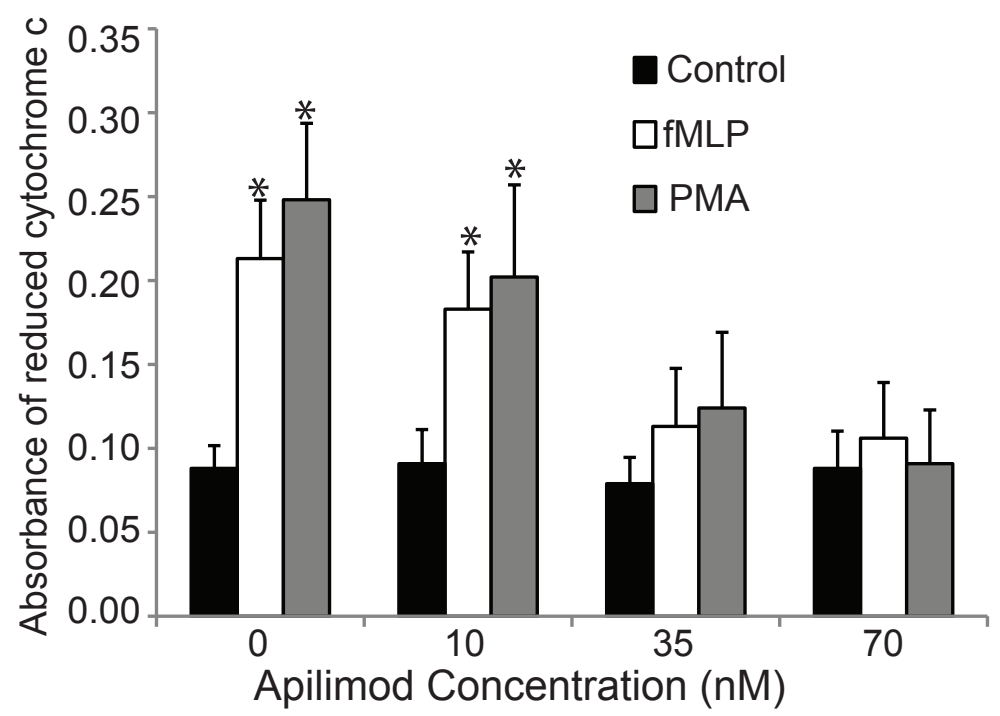

Figure 4.6: PIKfyve activity is essential for reactive oxygen species generation in neutrophils.

Neutrophils were treated with the indicated apilimod levels before being exposed to fMLP or PMA or remaining at a resting state. Reduction of cytochrome $\mathrm{C}$ was then measured by spectrophotometry as an indication of ROS production. Data is as the mean \pm SEM from $n=5$ independent experiments, followed by analysis with ANOVA and Tukey's post-hoc test. Asterisk indicates statistically significant difference $(p<0.05)$.

\subsubsection{PIKfyve activity is necessary for fMLP-induced Rac activation}

Our observations so far indicate that PIKfyve activity affects chemotaxis, activation of the NADPH oxidase and can impact phagocytosis. A common factor in all these processes is that they depend on the activation of the Rac GTPases (Anderson et al., 2010; C. D. Ellson, GobertGosse, et al., 2001; Knaus et al., 1991; Koh, Sun, Zhu, \& Glogauer, 2005; Roberts et al., 1999). Moreover, PIKfyve activity, through the action of MTMR3, has been linked to Rac GTPase activation to catalyse migration of non-leukocytes (Oppelt et al., 2013, 2014; Viaud et al., 2014). Finally, PtdIns(5)P has been shown to bind and stimulate Tiam1, a guanyl exchange factor for Rac (Viaud et al., 2014). Given all this, we postulated that PIKfyve activity was necessary to 
stimulate Rac GTPases in neutrophils. To test this hypothesis, we employed an affinity chromatography assay that uses a GST-chimeric protein of the PBD domain of p21-activated kinase to precipitate GTP-bound Rac, followed by Western blotting against the Rac1 GTPase. As expected, GST alone did not recover any Rac1 from cells stimulated with fMLP (Fig. 4.7). In addition, GST-PBD recovered little Rac1 in resting cells or cells treated with 50nM apilimodonly. In striking contrast, fMLP-treatment led to a strong recovery of the Rac1 GTPase, which was abolished by pre-treatment with 50nM apilimod (Fig. 4.7). Overall, these results support a model in which PIKfyve activity is necessary for Rac GTPase activation during neutrophil stimulation to control chemotaxis, ROS production and phagocytosis.

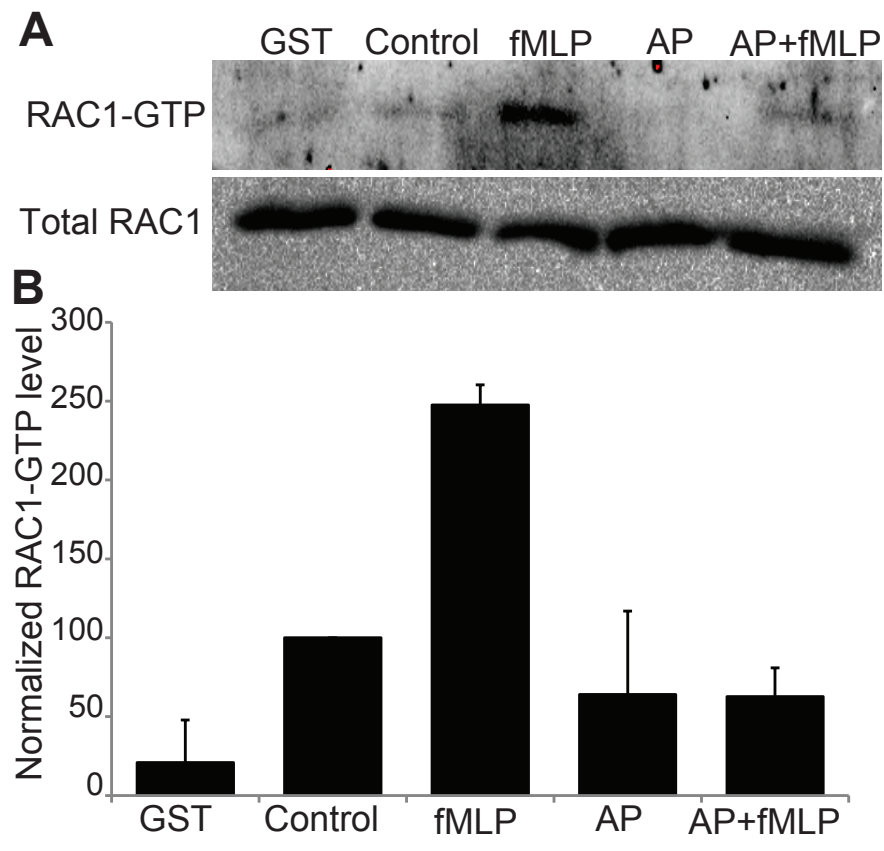

Figure 4.7: PIKfyve is essential to Rac GTPase stimulation in neutrophils.

A. Western blot against Rac1 GTPase after affinity precipitation for Rac1-GTP (top blot) from neutrophils treated as indicated and as described in Methods. GST alone is a negative control showing that Rac1-GTP is not interacting with the matrix or GST itself. Total Rac1 (10\% of the input for the pull-down) shows equal loading for each sample. B. Quantification of Rac1-GTP 
normalized to total Rac1 GTPase. Shown is the mean \pm SD from $n=3$ experiments. Asterisk $(*)$ indicates statistical significance against the control (resting cells) using Student's t-test $(p<0.05)$.

\subsection{Discussion}

Neutrophils are exceptionally important for the immune response, rapidly targeting sites of infection by chemotaxis and unleashing a series of attacks on pathogens including ROS generation, secretion of anti-bacterial peptides and engulfment and digestion of pathogens by phagocytosis (Mayadas et al., 2014; Nauseef \& Borregaard, 2014; Nordenfelt \& Tapper, 2011). These processes are dependent on and coordinated by a complex signaling network that employs small GTPases and phosphoinositide signals. However, the importance of PIKfyve activity, which synthesizes $\mathrm{PI}(3,5) \mathrm{P}_{2}$, and directly or indirectly, PtdIns(5)P, for neutrophil function remained unexplored. Here, we investigated this and found that PIKfyve activity is critical for neutrophils to perform chemotaxis, generate ROS and undertake phagosome fusion with lysosomes, but not for degranulation. Moreover, we propose a model by which phagosome maturation in neutrophils employs the $\mathrm{PI}(3,5) \mathrm{P}_{2}$ effector, TRPML1 to mediate lysosomal $\mathrm{Ca}^{2+}$ release and trigger phagosome-lysosome fusion, while chemotaxis and ROS proceed through PIKfyve-dependent activation of the Rac GTPase, likely through PtdIns(5)P.

\subsubsection{Impact of PIKfyve on neutrophil lysosomes and granules}

PIKfyve is well established to regulate lysosome morphology. In the absence of its activity, lysosomes in a multitude of cells, including the yeast vacuole, undergo massive enlargement (Ho et al., 2012; McCartney et al., 2014). However, to our knowledge and with the exception of platelet dense granules (Min et al., 2014b), lysosome-related organelles have not been 
examined for their susceptibility to enlarge in the absence of PIKfyve activity. Here, we show that neutrophils contain a distinct LAMP1-compartment that becomes engorged by acute impairment of PIKfyve activity. In contrast, none of the neutrophil granules that we examined underwent perceptible changes in morphology under acute inhibition of PIKfyve. In addition, primary granules could still secrete and fuse with phagosomes in neutrophils impaired for PIKfyve activity, which suggests that PIKfyve has little direct oversight in granule function in neutrophils. These results seem consistent with the normal biogenesis and stimulus-dependent secretion of alpha and dense granules found in platelets in mice carrying a platelet-specific PIKfyve gene deletion (Min et al., 2014b). Nevertheless, it remains possible that chronic deficiency of PIKfyve in neutrophils could impair biogenesis and function of the various neutrophil granules - this will require the generation of mice carrying a neutrophil-specific PIKfyve gene deletion.

\subsubsection{PIKfyve in phagosome maturation}

Neutrophils and macrophages have distinct phagosome maturation processes. While both employ phagosome-lysosome fusion, phagosomes in neutrophils also merge with primary granules, acquire a high concentration of ROS and do not acidify significantly (Nordenfelt \& Tapper, 2011; Tapper et al., 2002). Here, we showed that phagosomes enclosing IgG-coated particles require PIKfyve activity and $\mathrm{Ca}^{2+}$ to fuse with LAMP1-positive lysosomes in neutrophils. Importantly, ionomycin or the TRPML1 agonist, MLSA1, partly rescued phagosome acquisition of LAMP1 in PIKfyve-inhibited cells. This suggests that phagosome-lysosome fusion proceeds through a PI(3,5)P2 -dependent activation of TRPML1 to release lysosomal $\mathrm{Ca}^{2+}$ and trigger phagosome-lysosome fusion. These observations are consistent with our earlier findings in 
macrophages, which also require the PIKfyve-TRPML1-Ca ${ }^{2+}$ axis to trigger fusion between docked phagosomes and lysosomes (Dayam et al., 2015; G. H. E. Kim et al., 2014). In contrast, PIKfyve was not necessary for phagosome fusion with primary granules, suggesting a different mechanism for this process.

\subsubsection{PIKfyve activity is necessary for Rac activation in neutrophils}

Ablation of PIKfyve activity strongly impaired chemotaxis, ROS production and activation of Rac in response to $\mathrm{FMLP}$ signalling. Given that the NADPH oxidase, the primary source of ROS, and chemotaxis both employ Rac signaling, this suggests that PIKfyve impairs these functions by obstructing Rac function. The importance of PIKfyve for chemotaxis fits the observations by Oppelt et al. showing that PIKfyve regulates cell migration of fibroblasts and cancer cells (Oppelt et al., 2013, 2014). These authors suggested that PIKfyve collaborates with MTMR3 to generate PtdIns(5)P, which then stimulates Rac (Oppelt et al., 2013, 2014). More recently, Vlaud et al. showed that PtdIns(5)P binds preferentially to the C-terminal PH found of Tiam1 found within the canonical DH domain that serves as a hallmark for a large number of Rhofamily GEF proteins (Viaud et al., 2014). We envision that a similar process may be ensuing in stimulant-exposed neutrophils. In fact, neutrophils express various GEFs that contain a DH-PH domain that could conceivably be targeted by PtdIns(5)P, including Tiam1, Tiam2, P-Rex and Vav (Baker et al., 2016; Boespflug et al., 2014; Mizrahi et al., 2005; Welch et al., 2005).

Overall, we provide evidence that PIKfyve activity is necessary for a multitude of neutrophil functions. We posit that PIKfyve function is split between two signalling branches: i) a PI(3,5)P2 -dependent arm that governs phagosome maturation and lysosome activity through TRPML1 and ii) a PtdIns(5)P-dependent arm that regulates Rac, thus modulating the NADPH 
oxidase and chemotaxis. Unfortunately, attempts to biochemically measure $\mathrm{PI}(3,5) \mathrm{P} 2$ and PtdIns(5)P in neutrophils were not successful due to low ${ }^{3} \mathrm{H}-m y o$-inositol incorporation (Ho, Choy, \& Botelho, 2016). Thus, we could not test whether these PIs change during stimulation with $\mathrm{fMLP}$ or treatment with apilimod. Finally, it is noteworthy that apilimod was initially discovered as an anti-IL12/13 inhibitor and clinically-tested against Crohn's Disease (Cai et al.,

2013, 2014) - it is enticing to consider whether apilimod-dependent neutrophil suppression contributes to these clinically-relevant outcomes.

\subsection{Acknowledgements}

We thank the members of the Botelho lab for useful discussion of the manuscript. The vector encoding GST-PBD fusion protein was obtained from Addgene. 


\subsection{References}

Abo, A., Pick, E., Hall, A., Totty, N., Teahan, C. G., \& Segal, A. W. (1991). Activation of the NADPH oxidase involves the small GTP-binding protein p21rac1. Nature, 353(6345), 668-70. http://doi.org/10.1038/353668a0

Aderem, A. (2003). Phagocytosis and the inflammatory response. J.Infect.Dis., 187 Supp/(00221899 (Print)), S340-S345. http://doi.org/JID21046 [pii]\r10.1086/374747

Akerboom, J., Rivera, J. D. V., Rodríguez Guilbe, M. M., Malavé, E. C. A., Hernandez, H. H., Tian, L., ... Schreiter, E. R. (2009). Crystal structures of the GCaMP calcium sensor reveal the mechanism of fluorescence signal change and aid rational design. Journal of Biological Chemistry, 284(10), 6455-6464. http://doi.org/10.1074/jbc.M807657200

Akira, S., Uematsu, S., \& Takeuchi, O. (2006). Pathogen Recognition and Innate Immunity. Cell, 124(4), 783-801. http://doi.org/10.1016/j.cell.2006.02.015

Alberts, B., Johnson, A., Lewis, J., Raff, M., Roberts, K., And Walter, P., \& Alberts A.; Lewis, J. et al, B. . J. (2002). Molecular Biology of the cell. 4th edition. Amino Acids (Vol. 54).

Anderson, K. E., Chessa, T. A. M., Davidson, K., Henderson, R. B., Walker, S., Tolmachova, T., ... Hawkins, P. T. (2010). Ptdlns3P and Rac direct the assembly of the NADPH oxidase on a novel, pre-phagosomal compartment during FcR-mediated phagocytosis in primary mouse neutrophils. Blood, 116(23), 4978-4989. http://doi.org/10.1182/blood-2010-03-275602

Andrews, N. W. (2005). Membrane repair and immunological danger. EMBO Reports, 6(9), 826830. http://doi.org/10.1038/sj.embor.7400505

Colletti, G. A., Miedel, M. T., Andharia, N., Weisz, O. A., \& Kiselyov, K. (2012). Loss of Lysosomal Ion Channel Transient Receptor Potential Channel Mucolipin-1 ( TRPML1) Leads to 
Cathepsin, 1. http://doi.org/10.1074/jbc.M111.285536

Attout, T., Floto, A., \& Launay, P. (2014). Calcium channels in Fc receptor signaling. Current Topics in Microbiology and Immunology, 382, 95-110. http://doi.org/10.1007/978-3-31907911-0_5

Bajno, L., Peng, X. R., Schreiber, A. D., Moore, H. P., Trimble, W. S., \& Grinstein, S. (2000). Focal exocytosis of VAMP3-containing vesicles at sites of phagosome formation. The Journal of Cell Biology, 149(3), 697-706.

Baker, M. J., Pan, D., \& Welch, H. C. E. (2016). Small GTPases and their guanine-nucleotide exchange factors and GTPase-activating proteins in neutrophil recruitment. Current Opinion in Hematology, 23(1), 44-54. http://doi.org/10.1097/MOH.

Balla, T. (2013). Phosphoinositides: tiny lipids with giant impact on cell regulation. Physiological Reviews, 93(3), 1019-137. http://doi.org/10.1152/physrev.00028.2012

Barker, R. (1968). Chapter 3. Ecological Psychology. http://doi.org/10.1016/B978-1-85617-370$4.50005-1$

Beertsen, W., Willenborg, M., Everts, V., Zirogianni, A., Podschun, R., Schröder, B., ... Saftig, P. (2008). Impaired phagosomal maturation in neutrophils leads to periodontitis in lysosomal-associated membrane protein-2 knockout mice. Journal of Immunology (Baltimore, Md. : 1950), 180(1), 475-82.

Benard, V., Bohl, B. P., \& Bokoch, G. M. (1999). Characterization of Rac and Cdc42 activation in chemoattractant- stimulated human neutrophils using a novel assay for active GTPases. Journal of Biological Chemistry, 274(19), 13198-13204. http://doi.org/10.1074/jbc.274.19.13198 
Birkeland, H. C. G., \& Stenmark, H. (2004). Protein targeting to endosomes and phagosomes via FYVE and PX domains. Current Topics in Microbiology and Immunology, 282, 89-115.

Boespflug, N. D., Kumar, S., McAlees, J. W., Phelan, J. D., Grimes, H. L., Hoebe, K., ... Karp, C. L. (2014). ATF3 is a novel regulator of mouse neutrophil migration. Blood, 123(13), 2084-93. http://doi.org/10.1182/blood-2013-06-510909

Borghesi, L., \& Milcarek, C. (2007). Innate versus adaptive immunity: A paradigm past its prime? Cancer Research, 67(9), 3989-3993. http://doi.org/10.1158/0008-5472.CAN-07-0182

Braun, V., Fraisier, V., Raposo, G., Hurbain, I., Sibarita, J.-B., Chavrier, P., ... Niedergang, F. (2004). TI-VAMP/VAMP7 is required for optimal phagocytosis of opsonised particles in macrophages. The EMBO Journal, 23(21), 4166-76. http://doi.org/10.1038/sj.emboj.7600427

Bridges, D., Ma, J.-T., Park, S., Inoki, K., Weisman, L. S., \& Saltiel, A. R. (2012). Phosphatidylinositol 3,5-bisphosphate plays a role in the activation and subcellular localization of mechanistic target of rapamycin 1. Molecular Biology of the Cell, 23(15), 2955-62. http://doi.org/10.1091/mbc.E11-12-1034

Burgoyne, R. D., \& Clague, M. J. (2003). Calcium and calmodulin in membrane fusion. Biochimica et Biophysica Acta, 1641(2-3), 137-43.

Cai, X., Xu, Y., Cheung, A. K., Tomlinson, R. C., Alcázar-Román, A., Murphy, L., Huang, Q. (2013). PIKfyve, a class III PI Kinase, is the target of the small molecular IL-12/IL-23 inhibitor apilimod and a player in toll-like receptor signaling. Chemistry and Biology, 20(7), 912-921. http://doi.org/10.1016/j.chembiol.2013.05.010

Cai, X., Xu, Y., Kim, Y.-M., Loureiro, J., \& Huang, Q. (2014). PIKfyve, a class III lipid kinase, is 
required for TLR-induced type I IFN production via modulation of ATF3. Journal of Immunology (Baltimore, Md. : 1950), 192(7), 3383-9.

http://doi.org/10.4049/jimmunol.1302411

Carlier, M. F., Ducruix, A., \& Pantaloni, D. (1999). Signalling to actin: The Cdc42-N-WASP-Arp2/3 connection. Chemistry and Biology. http://doi.org/10.1016/S1074-5521(99)80107-0

Carstanjen, D., Yamauchi, A., Koornneef, A., Zang, H., Filippi, M.-D., Harris, C., Williams, D. a. (2005). Rac2 regulates neutrophil chemotaxis, superoxide production, and myeloid colony formation through multiple distinct effector pathways. Journal of Immunology (Baltimore, Md. : 1950), 174(8), 4613-20. http://doi.org/10.4049/jimmunol.174.8.4613

Chakrabarti, S., Andrade, L. O., \& Andrews, N. W. (2005). Trypanosoma cruzi invades synaptotagmin VII-deficient cells by a PI-3 kinase independent pathway. Molecular and Biochemical Parasitology, 141(1), 125-128.

http://doi.org/10.1016/j.molbiopara.2005.01.016

Cheng, X., Zhang, X., Gao, Q., Ali Samie, M., Azar, M., Tsang, W. L., Xu, H. (2014). The intracellular $\mathrm{Ca}(2+)$ channel MCOLN1 is required for sarcolemma repair to prevent muscular dystrophy. Nature Medicine, 20(10), 1187-92. http://doi.org/10.1038/nm.3611

Cieutat, A. M., Lobel, P., August, J. T., Kjeldsen, L., Sengeløv, H., Borregaard, N., \& Bainton, D. F. (1998). Azurophilic granules of human neutrophilic leukocytes are deficient in lysosomeassociated membrane proteins but retain the mannose 6-phosphate recognition marker. Blood, 91(3), 1044-58.

Clark, A. J., \& Petty, H. R. (2010). A cell permeant peptide containing the cytoplasmic tail sequence of Fc receptor type IIA reduces calcium signaling and phagolysosome formation 
in neutrophils. Cellular Immunology, 261(2), 153-158.

http://doi.org/10.1016/j.cellimm.2009.12.002

Colletti, G. A. (2011). STUDY OF TRPML CHANNELS REVEALS INSIGHT INTO ENDOCYTIC.

Coutinho, M. F., Prata, M. J., \& Alves, S. (2012). Mannose-6-phosphate pathway: A review on its role in lysosomal function and dysfunction. Molecular Genetics and Metabolism.

http://doi.org/10.1016/j.ymgme.2011.12.012

Cox, D., Berg, J. S., Cammer, M., Chinegwundoh, J. O., Dale, B. M., Cheney, R. E., \& Greenberg, S. (2002). Myosin X is a downstream effector of PI(3)K during phagocytosis. Nature Cell Biology, 4(7), 469-477. http://doi.org/10.1038/ncb805

Cuervo, A. M., \& Dice, J. F. (2000). Regulation of Lamp2a Levels in the Lysosomal Membrane. Traffic, 1, 570-583. http://doi.org/tra010707 [pii]

Cunningham, C. C., Vegners, R., Bucki, R., Funaki, M., Korde, N., Hartwig, J. H., Janmey, P. A. (2001). Cell permeant polyphosphoinositide-binding peptides that block cell motility and actin assembly. The Journal of Biological Chemistry, 276(46), 43390-9.

http://doi.org/10.1074/jbc.M105289200

Czibener, C., Sherer, N. M., Becker, S. M., Pypaert, M., Hui, E., Chapman, E. R., Andrews, N. W. (2006). Ca2+ and synaptotagmin VII-dependent delivery of lysosomal membrane to nascent phagosomes. The Journal of Cell Biology, 174(7), 997-1007. http://doi.org/10.1083/jcb.200605004

Davies, L. C., Jenkins, S. J., Allen, J. E., \& Taylor, P. R. (2013). Tissue-resident macrophages. Nature Immunology, 14(10), 986-95. http://doi.org/10.1038/ni.2705

Dayam, R. M., Saric, A., Shilliday, R. E., \& Botelho, R. J. (2015). The Phosphoinositide-Gated 
Lysosomal Ca(2+) Channel, TRPML1, Is Required for Phagosome Maturation. Traffic (Copenhagen, Denmark), 16(9), 1010-26. http://doi.org/10.1111/tra.12303

de Back, D. Z., Kostova, E. B., van Kraaij, M., van den Berg, T. K., \& van Bruggen, R. (2014). Of macrophages and red blood cells; A complex love story. Frontiers in Physiology. http://doi.org/10.3389/fphys.2014.00009 de Lartigue, J., Polson, H., Feldman, M., Shokat, K., Tooze, S. A., Urbé, S., \& Clague, M. J. (2009). PIKfyve regulation of endosome-linked pathways. Traffic (Copenhagen, Denmark), 10(7), 883-93.

Dell'Angelica, E. C., Mullins, C., Caplan, S., \& Bonifacino, J. S. (2000). Lysosome-related organelles. FASEB Journal : Official Publication of the Federation of American Societies for Experimental Biology, 14(10), 1265-78.

Di Paolo, G., \& De Camilli, P. (2006). Phosphoinositides in cell regulation and membrane dynamics. Nature, 443(7112), 651-657. http://doi.org/10.1038/nature05185

Dong, X., Shen, D., Wang, X., Dawson, T., Li, X., Zhang, Q., ... Chen, X. (2010). PI(3,5)P2 Mucolipin Ca2+ Controls Membrane Traffic by Direct Activation of Release Channels in the Endolysosome. Nature Communications, 1(4), 1-21. http://doi.org/10.1038/ncomms1037.PI)

Dong, X., Shen, D., Wang, X., Dawson, T., Li, X., Zhang, Q., Xu, H. (2010). PI(3,5)P(2) controls membrane trafficking by direct activation of mucolipin $\mathrm{Ca}(2+)$ release channels in the endolysosome. Nature Communications, 1, 38. http://doi.org/10.1038/ncomms1037

Dong, X., Wang, X., Shen, D., Chen, S., Liu, M., Wang, Y., Xu, H. (2009). Activating mutations of the TRPML1 channel revealed by proline-scanning mutagenesis. The Journal of Biological 
Chemistry, 284(46), 32040-52. http://doi.org/10.1074/jbc.M109.037184

Efeyan, A., Zoncu, R., \& Sabatini, D. M. (2012). Amino acids and mTORC1: From lysosomes to disease. Trends in Molecular Medicine. http://doi.org/10.1016/j.molmed.2012.05.007

El Benna, J., Ruedi, J. M., \& Babior, B. M. (1994). Cytosolic Guanine Nucleotide-binding Protein Rac2 Operates in Vivo as a Component of the Neutrophil Respiratory Burst Oxidase: Transfer of Rac2 and the cytosolic oxidase components p47phox and p67phox to the submembranous actin cytoskeleton during oxidase. Journal of Biological Chemistry, 269(9), 6729-6734.

Elhelu, M. A. (1983). The role of macrophages in immunology. Journal of the National Medical Association, 75(3), 314-7. Retrieved from http://www.pubmedcentral.nih.gov/articlerender.fcgi?artid=2561478\&tool=pmcentrez\&r endertype=abstract

Ellson, C. D., Anderson, K. E., Morgan, G., Chilvers, E. R., Lipp, P., Stephens, L. R., \& Hawkins, P. T. (2001). Phosphatidylinositol 3-phosphate is generated in phagosomal membranes. Current Biology : CB, 11(20), 1631-5.

Ellson, C. D., Gobert-Gosse, S., Anderson, K. E., Davidson, K., Erdjument-Bromage, H., Tempst, P., ... Stephens, L. R. (2001). PtdIns(3)P regulates the neutrophil oxidase complex by binding to the PX domain of p40(phox). Nature Cell Biology, 3(7), 679-82. http://doi.org/10.1038/35083076

Ellson, C., Davidson, K., Anderson, K., Stephens, L. R., \& Hawkins, P. T. (2006). PtdIns3P binding to the PX domain of p40phox is a physiological signal in NADPH oxidase activation. The EMBO Journal, 25(19), 4468-78. http://doi.org/10.1038/sj.emboj.7601346 
Eskelinen, E. L., Tanaka, Y., \& Saftig, P. (2003). At the acidic edge: Emerging functions for lysosomal membrane proteins. Trends in Cell Biology. http://doi.org/10.1016/S09628924(03)00005-9

Fairn, G. D., \& Grinstein, S. (2012). How nascent phagosomes mature to become phagolysosomes. Trends in Immunology, 33(8), 397-405. http://doi.org/10.1016/j.it.2012.03.003

Falkenburger, B. H., Jensen, J. B., Dickson, E. J., Suh, B. C., \& Hille, B. (2010). Phosphoinositides: lipid regulators of membrane proteins. J Physiol, 588(Pt 17), 3179-3185. http://doi.org/10.1113/jphysiol.2010.192153

Faurschou, M., \& Borregaard, N. (2003). Neutrophil granules and secretory vesicles in inflammation. Microbes and Infection, 5(14), 1317-1327.

http://doi.org/10.1016/j.micinf.2003.09.008

Faurschou, M., Sørensen, O. E., Johnsen, A. H., Askaa, J., \& Borregaard, N. (2002). Defensin-rich granules of human neutrophils: Characterization of secretory properties. Biochimica et Biophysica Acta - Molecular Cell Research, 1591(1-3), 29-35. http://doi.org/10.1016/S0167-4889(02)00243-4

Ferguson, C. J., Lenk, G. M., Jones, J. M., Grant, A. E., Winters, J. J., Dowling, J. J., ... Meisler, M. H. (2012). Neuronal expression of Fig4 is both necessary and sufficient to prevent spongiform neurodegeneration. Human Molecular Genetics, 21(16), 3525-34. http://doi.org/10.1093/hmg/dds179

Flannagan, R. S., Cosío, G., \& Grinstein, S. (2009). Antimicrobial mechanisms of phagocytes and bacterial evasion strategies. Nature Reviews. Microbiology, 7(5), 355-66. 
http://doi.org/10.1038/nrmicro2128

Flannagan, R. S., Jaumouillé, V., \& Grinstein, S. (2012). The cell biology of phagocytosis. Annual Review of Pathology, 7, 61-98. http://doi.org/10.1146/annurev-pathol-011811-132445

Fratti, R. A., Backer, J. M., Gruenberg, J., Corvera, S., \& Deretic, V. (2001). Role of phosphatidylinositol 3-kinase and Rab5 effectors in phagosomal biogenesis and mycobacterial phagosome maturation arrest. The Journal of Cell Biology, 154(3), 631-44. http://doi.org/10.1083/jcb.200106049

GarcI, E. (2002). Signal transduction during Fc receptor-mediated phagocytosis.

Gary, J. D., Wurmser, A. E., Bonangelino, C. J., Weisman, L. S., \& Emr, S. D. (1998). Fab1p is essential for PtdIns(3)P 5-kinase activity and the maintenance of vacuolar size and membrane homeostasis. Journal of Cell Biology, 143(1), 65-79.

http://doi.org/10.1083/jcb.143.1.65

Gerasimenko, J. V, Gerasimenko, O. V, \& Petersen, O. H. (2001). Membrane repair: Ca(2+)elicited lysosomal exocytosis. Current Biology : CB, 11(23), R971-4.

Gillooly, D. J., Morrow, I. C., Lindsay, M., Gould, R., Bryant, N. J., Gaullier, J. M., Stenmark, H. (2000). Localization of phosphatidylinositol 3-phosphate in yeast and mammalian cells. The EMBO Journal, 19(17), 4577-88. http://doi.org/10.1093/emboj/19.17.4577

Glogauer, M., Marchal, C. C., Zhu, F., Worku, A., Clausen, Foerster, I., Kwiatkowski, D. J. (2003). Rac1 deletion in mouse neutrophils has selective effects on neutrophil functions. J Immunol, 170(11), 5652-7. http://doi.org/10.4049/jimmunol.170.11.5652

Gold, E. S., Morrissette, N. S., Underhill, D. M., Guo, J., Bassetti, M., \& Aderem, a. (2000). Amphiphysin IIm, a novel amphiphysin II isoform, is required for macrophage 
phagocytosis. Immunity, 12(3), 285-292. http://doi.org/10.1016/S1074-7613(00)80181-8

Gordon-Smith, T. (2007). Red blood cells. Surgery (Oxford), 25(2), 57-60.

http://doi.org/10.1016/j.mpsur.2006.12.004

Gordon, S. (2003). Alternative activation of macrophages. Nat.Rev.Immunol., 3(1474-1733

(Print)), 23-35. http://doi.org/10.1038/nri978

Grosshans, B. L., Ortiz, D., \& Novick, P. (2006). Rabs and their effectors: achieving specificity in membrane traffic. Proceedings of the National Academy of Sciences of the United States of America, 103(32), 11821-7. http://doi.org/10.1073/pnas.0601617103

Hannigan, M., Zhan, L., Li, Z., Ai, Y., Wu, D., \& Huang, C.-K. (2002). Neutrophils lacking phosphoinositide 3-kinase gamma show loss of directionality during N-formyl-Met-LeuPhe-induced chemotaxis. Proceedings of the National Academy of Sciences of the United States of America, 99(6), 3603-8. http://doi.org/10.1073/pnas.052010699

Harrison, R. E., Bucci, C., Vieira, O. V, Schroer, T. A., \& Grinstein, S. (2003). Phagosomes fuse with late endosomes and/or lysosomes by extension of membrane protrusions along microtubules: role of Rab7 and RILP. Molecular and Cellular Biology, 23(18), 6494-506. http://doi.org/10.1128/MCB.23.18.6494-6506.2003

Hay, J. C. (2007). Calcium: a fundamental regulator of intracellular membrane fusion?, 8(3). http://doi.org/10.1038/sj.embor.7400921

Hazeki, K., Nigorikawa, K., Takaba, Y., Segawa, T., Nukuda, A., Masuda, A., Hazeki, O. (2012). Essential roles of PIKfyve and PTEN on phagosomal phosphatidylinositol 3-phosphate dynamics. FEBS Letters, 586(22), 4010-5. http://doi.org/10.1016/j.febslet.2012.09.043 Heyworth, P. G., Knaus, U. G., Settleman, J., Curnutte, J. T., \& Bokoch, G. M. (1993). Regulation 
of NADPH oxidase activity by Rac GTPase activating protein(s). Molecular Biology of the Cell, 4(11), 1217-1223. Retrieved from http://www.ncbi.nlm.nih.gov/pmc/articles/PMC275755/pdf/mbc00056-0140.pdf Ho, C. Y., Alghamdi, T. A., \& Botelho, R. J. (2012). Phosphatidylinositol-3,5-bisphosphate: no longer the poor PIP2. Traffic (Copenhagen, Denmark), 13(1), 1-8. http://doi.org/10.1111/j.1600-0854.2011.01246.x

Ho, C. Y., Choy, C. H., \& Botelho, R. J. (2016). Radiolabeling and Quantification of Cellular Levels of Phosphoinositides by High Performance Liquid Chromatography-coupled Flow Scintillation. Journal of Visualized Experiments : JoVE, (107). http://doi.org/10.3791/53529 Huotari, J., \& Helenius, A. (2011). Endosome maturation. The EMBO Journal, 30(17), 34813500. http://doi.org/10.1038/emboj.2011.286

Huynh, K. K., Eskelinen, E., Scott, C. C., Malevanets, A., Saftig, P., \& Grinstein, S. (2007). LAMP proteins are required for fusion of lysosomes with phagosomes, 26(2), 313-324. http://doi.org/10.1038/sj.emboj.7601511

Ikonomov, O. C., Sbrissa, D., Delvecchio, K., Xie, Y., Jin, J. P., Rappolee, D., \& Shisheva, A. (2011). The phosphoinositide kinase PIKfyve is vital in early embryonic development: Preimplantation lethality of PIKfyve-/- embryos but normality of PIKfyve+/- mice. Journal of Biological Chemistry, 286(15), 13404-13413. http://doi.org/10.1074/jbc.M111.222364 Ikonomov, O. C., Sbrissa, D., Fenner, H., \& Shisheva, A. (2009). PIKfyve-ArPIKfyve-Sac3 core complex: Contact sites and their consequence for Sac3 phosphatase activity and endocytic membrane homeostasis. Journal of Biological Chemistry, 284(51), 35794-35806. http://doi.org/10.1074/jbc.M109.037515 
Ikonomov, O. C., Sbrissa, D., Foti, M., Carpentier, J.-L., \& Shisheva, A. (2003). PIKfyve controls fluid phase endocytosis but not recycling/degradation of endocytosed receptors or sorting of procathepsin D by regulating multivesicular body morphogenesis. Molecular Biology of the Cell, 14(11), 4581-91. http://doi.org/10.1091/mbc.E03-04-0222

Ikonomov, O. C., Sbrissa, D., Mlak, K., Kanzaki, M., Pessin, J., \& Shisheva, A. (2002). Functional dissection of lipid and protein kinase signals of PIKfyve reveals the role of PtdIns 3,5-P2 production for endomembrane integrity. The Journal of Biological Chemistry, 277(11), 9206-11. http://doi.org/10.1074/jbc.M108750200

Ikonomov, O. C., Sbrissa, D., \& Shisheva, A. (2001). Mammalian cell morphology and endocytic membrane homeostasis require enzymatically active phosphoinositide 5-kinase PIKfyve. The Journal of Biological Chemistry, 276(28), 26141-7.

http://doi.org/10.1074/jbc.M101722200

Italiani, P., \& Boraschi, D. (2014). From monocytes to M1/M2 macrophages: Phenotypical vs. functional differentiation. Frontiers in Immunology, 5(OCT), 1-22. http://doi.org/10.3389/fimmu.2014.00514

Jaconi, M. E., Lew, D. P., Carpentier, J. L., Magnusson, K. E., Sjögren, M., \& Stendahl, O. (1990). Cytosolic free calcium elevation mediates the phagosome-lysosome fusion during phagocytosis in human neutrophils. The Journal of Cell Biology, 110(5), 1555-64. Jankowski, A., Scott, C. C., \& Grinstein, S. (2002). Determinants of the phagosomal pH in neutrophils. Journal of Biological Chemistry, 277(8), 6059-6066. http://doi.org/10.1074/jbc.M110059200 Jayachandran, R., Sundaramurthy, V., Combaluzier, B., Mueller, P., Korf, H., Huygen, K., Pieters, 
J. (2007). Survival of mycobacteria in macrophages is mediated by coronin 1-dependent activation of calcineurin. Cell, 130(1), 37-50. http://doi.org/10.1016/j.cell.2007.04.043

Jefferies, H. B. J., Cooke, F. T., Jat, P., Boucheron, C., Koizumi, T., Hayakawa, M., Parker, P. J. (2008). A selective PIKfyve inhibitor blocks PtdIns(3,5)P(2) production and disrupts endomembrane transport and retroviral budding. EMBO Reports, 9(2), 164-170. http://doi.org/10.1038/sj.embor.7401155

Karacsonyi, C., San Miguel, A., \& Puertollano, R. (2007). Mucolipin-2 localizes to the Arf6associated pathway and regulates recycling of GPI-APs. Traffic, 8(10), 1404-1414. http://doi.org/10.1111/j.1600-0854.2007.00619.x

Kawano, Y., Kaneko-Kawano, T., Shimamoto, K., Hückelhoven, R., \& Zhang, Y. (2014). Rho family GTPase-dependent immunity in plants and animals. Frontiers in Plant Science, 5, 522. http://doi.org/10.3389/fpls.2014.00522

Kielian, M. C., \& Cohn, Z. A. (1980). Phagosome-lysosome fusion. Characterization of intracellular membrane fusion in mouse macrophages. The Journal of Cell Biology, 85(3), $754-65$.

Kim, G. H. E., Dayam, R. M., Prashar, A., Terebiznik, M., \& Botelho, R. J. (2014). PIKfyve Inhibition Interferes with Phagosome and Endosome Maturation in Macrophages, (3), 121. http://doi.org/10.1111/tra.12199

Kim, H. J., Soyombo, A. A., Tjon-Kon-Sang, S., So, I., \& Muallem, S. (2009). The Ca2+ channel TRPML3 regulates membrane trafficking and autophagy. Traffic, 10(8), 1157-1167. http://doi.org/10.1111/j.1600-0854.2009.00924.x

Kinchen, J. M., \& Ravichandran, K. S. (2008). Phagosome maturation: going through the acid 
test. Nature Reviews. Molecular Cell Biology, 9(10), 781-95.

http://doi.org/10.1038/nrm2515

Kiselyov, K. K., Ahuja, M., Rybalchenko, V., Patel, S., \& Muallem, S. The intracellular Ca ${ }^{2+}$ channels of membrane traffic. Channels (Austin, Tex.), 6(5), 344-51.

http://doi.org/10.4161/chan.21723

Klebanoff, S. J. (1999). Myeloperoxidase. Proc Assoc Am Physicians, 111(5), 383-389.

Knaus, U. G., Heyworth, P. G., Evans, T., Curnutte, J. T., \& Bokoch, G. M. (1991). Regulation of phagocyte oxygen radical production by the GTP-binding protein Rac 2. Science (New York, N.Y.), 254(5037), 1512-5.

Koh, A. L. Y., Sun, C. X., Zhu, F., \& Glogauer, M. (2005). The role of Rac1 and Rac2 in bacterial killing. Cellular Immunology, 235(2), 92-97. http://doi.org/10.1016/j.cellimm.2005.07.005

Kornfeld, S., \& Mellman, I. (1989). The biogenesis of lysosomes. Annual Review of Cell Biology, 5, 483-525. http://doi.org/10.1146/annurev.cb.05.110189.002411

Krajcovic, M., Krishna, S., Akkari, L., Joyce, J. a, \& Overholtzer, M. (2013). mTOR regulates phagosome and entotic vacuole fission. Molecular Biology of the Cell, 24(23), 3736-3745. http://doi.org/10.1091/mbc.E13-07-0408

Lee, W. L., Harrison, R. E., \& Grinstein, S. (2003a). Phagocytosis by neutrophils. Microbes and Infection. http://doi.org/10.1016/j.micinf.2003.09.014

Lee, W. L., Harrison, R. E., \& Grinstein, S. (2003b). Phagocytosis by neutrophils. Microbes and Infection, 5(14), 1299-1306.

Lemmon, M. A. (2008). Membrane recognition by phospholipid-binding domains. Nature Reviews. Molecular Cell Biology, 9(2), 99-111. http://doi.org/10.1038/nrm2328 
Lew, P. D., Monod, A., Waldvogel, F. A., Dewald, B., Baggiolini, M., \& Pozzan, T. (1986).

Quantitative analysis of the cytosolic free calcium dependency of exocytosis from three subcellular compartments in intact human neutrophils. The Journal of Cell Biology, 102(6), 2197-204.

Li, X., Wang, X., Zhang, X., Zhao, M., Tsang, W. L., Zhang, Y., ... Xu, H. (2013). Genetically encoded fluorescent probe to visualize intracellular phosphatidylinositol 3,5-bisphosphate localization and dynamics. Proceedings of the National Academy of Sciences of the United States of America, 110(52), 21165-70. http://doi.org/10.1073/pnas.1311864110

Lietha, D. (2011). Phosphoinositides - The Seven Species: Conversion and Cellular Roles. In Encyclopedia of Life Sciences (pp. 1-11). http://doi.org/10.1002/9780470015902.a0023177

Madsen, S. J., Baek, S. K., Makkouk, A. R., Krasieva, T., \& Hirschberg, H. (2012). Macrophages as cell-based delivery systems for nanoshells in photothermal therapy. Annals of Biomedical Engineering, 40(2), 507-515. http://doi.org/10.1007/s10439-011-0415-1

Makita, N., Hizukuri, Y., Yamashiro, K., \& Murakawa, M. (2014). IL-10 enhances the phenotype of M2 macrophages induced by IL-4 and confers the ability to increase eosinophil migration, 27(3), 131-141. http://doi.org/10.1093/intimm/dxu090

Malik, Z. A., Denning, G. M., \& Kusner, D. J. (2000). Inhibition of Ca(2+) signaling by Mycobacterium tuberculosis is associated with reduced phagosome-lysosome fusion and increased survival within human macrophages. The Journal of Experimental Medicine, 191(2), 287-302.

Malik, Z. A., Thompson, C. R., Hashimi, S., Porter, B., Iyer, S. S., \& Kusner, D. J. (2003). Cutting 
edge: Mycobacterium tuberculosis blocks Ca2+ signaling and phagosome maturation in human macrophages via specific inhibition of sphingosine kinase. Journal of Immunology (Baltimore, Md. : 1950), 170(6), 2811-5.

Martin, S., Harper, C. B., May, L. M., Coulson, E. J., Meunier, F. A., \& Osborne, S. L. (2013). Inhibition of PIKfyve by YM-201636 dysregulates autophagy and leads to apoptosisindependent neuronal cell death. PloS One, 8(3), e60152. http://doi.org/10.1371/journal.pone.0060152

Mayadas, T. N., Cullere, X., \& Lowell, C. A. (2014). The multifaceted functions of neutrophils. Annual Review of Pathology, 9, 181-218.

McCartney, A. J., Zhang, Y., \& Weisman, L. S. (2014). Phosphatidylinositol 3,5-bisphosphate: low abundance, high significance. BioEssays : News and Reviews in Molecular, Cellular and Developmental Biology, 36(1), 52-64. http://doi.org/10.1002/bies.201300012

Mellman, I. (1996). Endocytosis and molecular sorting. Annual Review of Cell and Developmental Biology, 12, 575-625. http://doi.org/10.1146/annurev.cellbio.12.1.575

Min, S. H., Suzuki, A., Stalker, T. J., Zhao, L., Wang, Y., McKennan, C., ... Abrams, C. S. (2014a). Loss of PIKfyve in platelets causes a lysosomal disease leading to inflammation and thrombosis in mice. Nature Communications, 5(May), 4691. http://doi.org/10.1038/ncomms5691

Min, S. H., Suzuki, A., Stalker, T. J., Zhao, L., Wang, Y., McKennan, C., Abrams, C. S. (2014b). Loss of PIKfyve in platelets causes a lysosomal disease leading to inflammation and thrombosis in mice. Nature Communications, 5, 4691. http://doi.org/10.1038/ncomms5691

Minakami, R., Maehara, Y., Kamakura, S., Kumano, O., Miyano, K., \& Sumimoto, H. (2010). 
Membrane phospholipid metabolism during phagocytosis in human neutrophils. Genes to Cells : Devoted to Molecular \& Cellular Mechanisms, 15(5), 409-24.

Mitchell, M. A., Huang, M. M., Chien, P., Indik, Z. K., Pan, X. Q., \& Schreiber, A. D. (1994). Substitutions and deletions in the cytoplasmic domain of the phagocytic receptor Fc gamma RIIA: effect on receptor tyrosine phosphorylation and phagocytosis. Blood, 84(6), 1753-9. Retrieved from http://www.ncbi.nlm.nih.gov/pubmed/7521687

Mizrahi, A., Molshanski-Mor, S., Weinbaum, C., Zheng, Y., Hirshberg, M., \& Pick, E. (2005). Activation of the phagocyte NADPH oxidase by Rac Guanine nucleotide exchange factors in conjunction with ATP and nucleoside diphosphate kinase. The Journal of Biological Chemistry, 280(5), 3802-11. http://doi.org/10.1074/jbc.M410257200

Mrakovic, A., Kay, J. G., Furuya, W., Brumell, J. H., \& Botelho, R. J. (2012). Rab7 and Arl8 GTPases are Necessary for Lysosome Tubulation in Macrophages. Traffic, 13(12), 16671679. http://doi.org/10.1111/tra.12003

Munoz, C., Almilaji, A., Setiawan, I., Föller, M., \& Lang, F. (2013). Up-regulation of the inwardly rectifying $\mathrm{K}^{+}$channel Kir2.1 (KCNJ2) by protein kinase B (PKB/Akt) and PIKfyve. The Journal of Membrane Biology, 246(3), 189-97. http://doi.org/10.1007/s00232-012-9520-9

Muraille, E., Leo, O., \& Moser, M. (2014). Th1/Th2 paradigm extended: Macrophage polarization as an unappreciated pathogen-driven escape mechanism? Frontiers in Immunology. http://doi.org/10.3389/fimmu.2014.00603

Murray, P. J., \& Wynn, T. A. (2011). Protective and pathogenic functions of macrophage subsets. Nature Reviews Immunology, 11(11), 723-737. http://doi.org/Doi 10.1038/Nri3073 
Nauseef, W. M., \& Borregaard, N. (2014). Neutrophils at work. Nature Immunology, 15(7), 60211. http://doi.org/10.1038/ni.2921

Newton, K., \& Dixit, V. M. (2012). Signaling in Innate Immunity and Inflammation.

Nicot, A.-S., Fares, H., Payrastre, B., Chisholm, A. D., Labouesse, M., \& Laporte, J. (2006). The phosphoinositide kinase PIKfyve/Fab1p regulates terminal lysosome maturation in Caenorhabditis elegans. Molecular Biology of the Cell, 17, 3062-3074. http://doi.org/10.1091/mbc.E05-12-1120

Niessen, H. W., Kuijpers, T. W., Roos, D., \& Verhoeven, A. J. (1991). Release of azurophilic granule contents in fMLP-stimulated neutrophils requires two activation signals, one of which is a rise in cytosolic free Ca2+. Cellular Signalling, 3(6), 625-33.

Nordenfelt, P., \& Tapper, H. (2011). Phagosome dynamics during phagocytosis by neutrophils. Journal of Leukocyte Biology, 90(August), 271-284. http://doi.org/10.1189/jlb.0810457 Nordmann, M., Cabrera, M., Perz, A., Br??cker, C., Ostrowicz, C., Engelbrecht-Vandr??, S., \& Ungermann, C. (2010). The Mon1-Ccz1 complex is the GEF of the late endosomal Rab7 homolog Ypt7. Current Biology, 20(18), 1654-1659. http://doi.org/10.1016/j.cub.2010.08.002

Oppelt, A., Haugsten, E. M., Zech, T., Danielsen, H. E., Sveen, A., Lobert, V. H., Wesche, J. (2014). PIKfyve, MTMR3 and their product PtdIns5P regulate cancer cell migration and invasion through activation of Rac1. The Biochemical Journal, 461(3), 383-90. http://doi.org/10.1042/BJ20140132

Oppelt, A., Lobert, V. H., Haglund, K., Mackey, A. M., Rameh, L. E., Liestøl, K., Wesche, J. (2013). Production of phosphatidylinositol 5-phosphate via PIKfyve and MTMR3 regulates cell 
migration. EMBO Reports, 14(1), 57-64. http://doi.org/10.1038/embor.2012.183

Osborne, S. L., Wen, P. J., Boucheron, C., Nguyen, H. N., Hayakawa, M., Kaizawa, H., Meunier, F. a. (2008). PIKfyve negatively regulates exocytosis in neurosecretory cells. Journal of Biological Chemistry, 283(5), 2804-2813. http://doi.org/10.1074/jbc.M704856200

Pakladok, T., Almilaji, A., Munoz, C., Alesutan, I., \& Lang, F. (2013). PIKfyve sensitivity of hERG channels. Cellular Physiology and Biochemistry : International Journal of Experimental Cellular Physiology, Biochemistry, and Pharmacology, 31(6), 785-94. http://doi.org/10.1159/000350096

Palmer, C., Diehn, M., Alizadeh, A. a, \& Brown, P. O. (2006). Cell-type specific gene expression profiles of leukocytes in human peripheral blood. BMC Genomics, 7, 115. http://doi.org/10.1186/1471-2164-7-115

Pesanti, E. L., \& Axline, S. G. (1975). Phagolysosome formation in normal and colchicine-treated macrophages. The Journal of Experimental Medicine, 142(4), 903-13.

Pryor, P. R., Mullock, B. M., Bright, N. A., Gray, S. R., \& Luzio, J. P. (2000). The role of intraorganellar $\mathrm{Ca}(2+)$ in late endosome-lysosome heterotypic fusion and in the reformation of lysosomes from hybrid organelles. The Journal of Cell Biology, 149(5), 1053-62.

Pryor, P. R., Mullock, B. M., Bright, N. A., Gray, S. R., \& Luzio, J. P. (2000). The role of intraorganellar $\mathrm{Ca} 2+$ in late endosome-lysosome heterotypic fusion and in the reformation of lysosomes from hybrid organelles. Journal of Cell Biology, 149(5), 1053-1062. http://doi.org/10.1083/jcb.149.5.1053

Pryor, P. R., Mullock, B. M., Bright, N. A., Gray, S. R., \& Luzio, J. P. (2000). The Role of 
Intraorganellar Ca 2 z in Late Endosome - Lysosome Heterotypic Fusion and in the Reformation of Lysosomes from Hybrid Organelles, 149(5), 1053-1062.

Pryor, P. R., Reimann, F., Gribble, F. M., \& Luzio, J. P. (2006). Mucolipin-1 Is a Lysosomal Membrane Protein Required for Intracellular Lactosylceramide Traffic, 1388-1398. http://doi.org/10.1111/j.1600-0854.2006.00475.x

Reddy, A., Caler, E. V, \& Andrews, N. W. (2001). Plasma membrane repair is mediated by $\mathrm{Ca}(2+)$-regulated exocytosis of lysosomes. Cell, 106(2), 157-69.

Roberts, A. W., Kim, C., Zhen, L., Lowe, J. B., Kapur, R., Petryniak, B., Williams, D. A. (1999). Deficiency of the Hematopoietic Cell-Specific Rho Family GTPase Rac2 Is Characterized by Abnormalities in Neutrophil Function and Host Defense. Immunity, 10(2), 183-196. http://doi.org/10.1016/S1074-7613(00)80019-9

Rohatgi, R., Ho, H. Y. H., \& Kirschner, M. W. (2000). Mechanism of N-WASP activation by CDC42 and phosphatidylinositol 4,5-bisphosphate. Journal of Cell Biology, 150(6), 1299-1309. http://doi.org/10.1083/jcb.150.6.1299

Rong, Y., Liu, M., Ma, L., Du, W., Zhang, H., Tian, Y., Yu, L. (2012). Clathrin and phosphatidylinositol-4,5-bisphosphate regulate autophagic lysosome reformation. Nature Cell Biology, 14(9), 924-934. http://doi.org/10.1038/ncb2557

Rutherford, A. C., Traer, C., Wassmer, T., Pattni, K., Bujny, M. V, Carlton, J. G., Cullen, P. J. (2006). The mammalian phosphatidylinositol 3-phosphate 5-kinase (PIKfyve) regulates endosome-to-TGN retrograde transport. Journal of Cell Science, 119(Pt 19), 3944-57. http://doi.org/10.1242/jcs.03153

Saarikangas, J., Zhao, H., \& Lappalainen, P. (2010). Regulation of the actin cytoskeleton-plasma 
membrane interplay by phosphoinositides. Physiological Reviews, 90, 259-289.

http://doi.org/10.1152/physrev.00036.2009

Sadhu, C., Masinovsky, B., Dick, K., Sowell, C. G., \& Staunton, D. E. (2003). Essential role of phosphoinositide 3-kinase delta in neutrophil directional movement. Journal of Immunology (Baltimore, Md. : 1950), 170(5), 2647-54.

Saftig, P., \& Klumperman, J. (2009). Lysosome biogenesis and lysosomal membrane proteins: trafficking meets function. Nature Reviews. Molecular Cell Biology, 10(9), 623-635. http://doi.org/10.1038/nrm2745

Samie, M. A., \& Xu, H. (n.d.). Lysosomal Exocytosis and Lipid Storage Disorders.

Samie, M., Wang, X., Zhang, X., Goschka, A., Li, X., Cheng, X., Ferrer, M. (2013). Article A TRP Channel in the Lysosome Regulates Large Particle Phagocytosis via Focal Exocytosis. Developmental Cell, 1-14. http://doi.org/10.1016/j.devcel.2013.08.003

Sbrissa, D., Ikonomov, O. C., Filios, C., Delvecchio, K., \& Shisheva, A. (2012). Functional dissociation between PIKfyve-synthesized Ptdlns5P and PI(3,5)P2 by means of the PIKfyve inhibitor YM201636. American Journal of Physiology. Cell Physiology, 303(4), C436-46. http://doi.org/10.1152/ajpcell.00105.2012

Sbrissa, D., Ikonomov, O. C., \& Shisheva, A. (2000). PIKfyve lipid kinase is a protein kinase: Downregulation of 5 ???-phosphoinositide product formation by autophosphorylation. Biochemistry, 39(51), 15980-15989. http://doi.org/10.1021/bi001897f

Scott, C. C., Botelho, R. J., \& Grinstein, S. (2003). Phagosome maturation: A few bugs in the system. Journal of Membrane Biology. http://doi.org/10.1007/s00232-002-2008-2

Segal, A. W. (2008). The function of the NADPH oxidase of phagocytes and its relationship to 
other NOXs in plants, invertebrates, and mammals. International Journal of Biochemistry and Cell Biology. http://doi.org/10.1016/j.biocel.2007.10.003

Segal, A. W., Dorling, J., \& Coade, S. (1980). Kinetics of fusion of the cytoplasmic granules with phagocytic vacuoles in human polymorphonuclear leukocytes. Biochemical and morphological studies. Journal of Cell Biology, 85(1), 42-59. http://doi.org/10.1083/jcb.85.1.42

Sengeløv, H., Kjeldsen, L., Diamond, M. S., Springer, T. a, \& Borregaard, N. (1993). Subcellular localization and dynamics of Mac-1 (alpha m beta 2) in human neutrophils. The Journal of Clinical Investigation, 92(3), 1467-1476. http://doi.org/10.1172/JCI116724

Shen, D., Wang, X., Li, X., Zhang, X., Yao, Z., Dibble, S., ... Xu, H. (2012). Lipid storage disorders block lysosomal trafficking by inhibiting a TRP channel and lysosomal calcium release. Nature Communications, 3, 731. http://doi.org/10.1038/ncomms1735

Sheshachalam, A., Srivastava, N., Mitchell, T., Lacy, P., \& Eitzen, G. (2014). Granule protein processing and regulated secretion in neutrophils. Frontiers in Immunology, 5, 448. http://doi.org/10.3389/fimmu.2014.00448

Shisheva, A., Sbrissa, D., \& Ikonomov, O. (2015). Plentiful PtdIns5P from scanty PI(3,5)P2 or from ample PtdIns? PIKfyve-dependent models: Evidence and speculation (response to: DOI 10.1002/bies.201300012). BioEssays : News and Reviews in Molecular, Cellular and Developmental Biology, 37(3), 267-77. http://doi.org/10.1002/bies.201400129

Sobota, A., Strzelecka-Kiliszek, A., Gładkowska, E., Yoshida, K., Mrozińska, K., \& Kwiatkowska, K. (2005). Binding of IgG-opsonized particles to Fc gamma R is an active stage of phagocytosis that involves receptor clustering and phosphorylation. Journal of Immunology (Baltimore, 
Md. : 1950), 175, 4450-4457. http://doi.org/10.4049/jimmunol.175.7.4450

Soyombo, A. A., Tjon-kon-sang, S., Rbaibi, Y., Bashllari, E., Muallem, S., Soyombo, A. A., Bisceglia, J. (2006). TRP-ML1 Regulates Lysosomal pH and Acidic Lysosomal Lipid Hydrolytic Activity *. http://doi.org/10.1074/jbc.M508211200

Steinberg, B. E., \& Grinstein, S. (2008). Pathogen destruction versus intracellular survival: The role of lipids as phagosomal fate determinants. Journal of Clinical Investigation. http://doi.org/10.1172/JCl35433

Su, D. L., Lu, Z. M., Shen, M. N., Li, X., \& Sun, L. Y. (2012). Roles of pro- and anti-inflammatory cytokines in the pathogenesis of SLE. Journal of Biomedicine and Biotechnology. http://doi.org/10.1155/2012/347141

Tapper, H., Furuya, W., \& Grinstein, S. (2002). Localized exocytosis of primary (lysosomal) granules during phagocytosis: role of $\mathrm{Ca2+-dependent} \mathrm{tyrosine} \mathrm{phosphorylation} \mathrm{and}$ microtubules. Journal of Immunology (Baltimore, Md. : 1950), 168(10), 5287-96.

Teo, Z. P., \& Hughes, D. (2003). The Role of Macrophages in Apoptosis : Initiator, Regulator , Scavenger. Reviews in Undergraduate Research, 2, 7-11.

Thompson, E. G., Schaheen, L., Dang, H., \& Fares, H. (2007). Lysosomal trafficking functions of mucolipin-1 in murine macrophages. BMC Cell Biology, 8, 54. http://doi.org/10.1186/14712121-8-54

Treusch, S., Knuth, S., Slaugenhaupt, S. a, Goldin, E., Grant, B. D., \& Fares, H. (2004). Caenorhabditis elegans functional orthologue of human protein h-mucolipin-1 is required for lysosome biogenesis. Proceedings of the National Academy of Sciences of the United States of America, 101(13), 4483-4488. http://doi.org/10.1073/pnas.0400709101 
Tsuruta, F., Green, E. M., Rousset, M., \& Dolmetsch, R. E. (2009). PIKfyve regulates CaV1.2 degradation and prevents excitotoxic cell death. The Journal of Cell Biology, 187(2), 27994. http://doi.org/10.1083/jcb.200903028

Tuosto, L., Capuano, C., Muscolini, M., Santoni, A., \& Galandrini, R. (2015). The multifaceted role of PIP2 in leukocyte biology. Cellular and Molecular Life Sciences : CMLS, 72(23), 446174. http://doi.org/10.1007/s00018-015-2013-0

Underhill, D. M., \& Ozinsky, A. (2002). Phagocytosis of microbes: complexity in action. Annual Review of Immunology, 20, 825-52.

http://doi.org/10.1146/annurev.immunol.20.103001.114744

Urbé, S., Mills, I. G., Stenmark, H., Kitamura, N., \& Clague, M. J. (2000). Endosomal localization and receptor dynamics determine tyrosine phosphorylation of hepatocyte growth factorregulated tyrosine kinase substrate. Molecular and Cellular Biology, 20(20), 7685-92.

Van Gisbergen, K. P. J. M., Geijtenbeek, T. B. H., \& Van Kooyk, Y. (2005). Close encounters of neutrophils and DCs. Trends in Immunology. http://doi.org/10.1016/j.it.2005.09.007 van Gisbergen, P. A. C., Li, M., Wu, S. Z., \& Bezanilla, M. (2012). Class II formin targeting to the cell cortex by binding PI(3,5)P 2 is essential for polarized growth. Journal of Cell Biology, 198(2), 235-250. http://doi.org/10.1083/jcb.201112085

Venkatachalam, K., Wong, C., \& Zhu, M. X. (2014). The role of TRPMLs in endolysosomal trafficking and function. Cell Calcium. http://doi.org/10.1016/j.ceca.2014.10.008

Vergne, I., Chua, J., \& Deretic, V. (2003). Tuberculosis toxin blocking phagosome maturation inhibits a novel Ca2+/calmodulin-PI3K hVPS34 cascade. The Journal of Experimental Medicine, 198(4), 653-9. http://doi.org/10.1084/jem.20030527 
Vergne, I., Fratti, R. A., Hill, P. J., Chua, J., Belisle, J., \& Deretic, V. (2004). Mycobacterium tuberculosis Phagosome Maturation Arrest : Mycobacterial Phosphatidylinositol Analog Phosphatidylinositol Mannoside Stimulates Early Endosomal Fusion, 15(February), 751760. http://doi.org/10.1091/mbc.E03

Viaud, J., Lagarrigue, F., Ramel, D., Allart, S., Chicanne, G., Ceccato, L., ... Gaits-lacovoni, F. (2014). Phosphatidylinositol 5-phosphate regulates invasion through binding and activation of Tiam1. Nature Communications, 5(May), 4080. http://doi.org/10.1038/ncomms5080

Vieira, O. V., Botelho, R. J., Rameh, L., Brachmann, S. M., Matsuo, T., Davidson, H. W., ... Grinstein, S. (2001). Distinct roles of class I and class III phosphatidylinositol 3-kinases in phagosome formation and maturation. Journal of Cell Biology, 155(1), 19-25. http://doi.org/10.1083/jcb.200107069

Vieira, O. V, Botelho, R. J., \& Grinstein, S. (2002). Phagosome maturation: aging gracefully. The Biochemical Journal, 366(Pt 3), 689-704. http://doi.org/10.1042/BJ20020691

Vieira, O. V, Botelho, R. J., Rameh, L., Brachmann, S. M., Matsuo, T., Davidson, H. W., ... Grinstein, S. (2001). Distinct roles of class I and class III phosphatidylinositol 3-kinases in phagosome formation and maturation. The Journal of Cell Biology, 155(1), 19-25. http://doi.org/10.1083/jcb.200107069

Wang, W., Zhang, X., Gao, Q., \& Xu, H. (2014). TRPML1: an ion channel in the lysosome. Handbook of Experimental Pharmacology, 222, 631-45. http://doi.org/10.1007/978-3-642$54215-2 \_24$

Welch, H. C. E., Condliffe, A. M., Milne, L. J., Ferguson, G. J., Hill, K., Webb, L. M. C., Stephens, L. 
R. (2005). P-Rex1 Regulates Neutrophil Function. Current Biology (Vol. 15).

http://doi.org/10.1016/j.cub.2005.09.050

Wick, M. J. (2003). The role of dendritic cells in the immune response to Salmonella.

Immunology Letters, 85(2), 99-102. http://doi.org/DOI: 10.1016/S0165-2478(02)00230-4

Wong, C. O., Li, R., Montell, C., \& Venkatachalam, K. (2012). Drosophila TRPML is required for TORC1 activation. Current Biology, 22(17), 1616-1621.

http://doi.org/10.1016/j.cub.2012.06.055

Xu, Y., Hortsman, H., Seet, L., Wong, S. H., \& Hong, W. (2001). SNX3 regulates endosomal function through its PX-domain-mediated interaction with PtdIns(3)P. Nature Cell Biology, 3(7), 658-66. http://doi.org/10.1038/35083051

Yu, L., McPhee, C. K., Zheng, L., Mardones, G. A., Rong, Y., Peng, J., Lenardo, M. J. (2010). Termination of autophagy and reformation of lysosomes regulated by mTOR. Nature, 465(7300), 942-6. http://doi.org/10.1038/nature09076

Zeevi, D. A., Frumkin, A., \& Bach, G. (2007). TRPML and lysosomal function. Biochimica et Biophysica Acta, 1772(8), 851-8. http://doi.org/10.1016/j.bbadis.2007.01.004

Zhang, J.-M., \& An, J. (2007). Cytokines, Inflammation and Pain. Int Anesthesiol Clin., 45(2), 2737. http://doi.org/10.1097/AIA.0b013e318034194e.

Zhang, Y., Zolov, S. N., Chow, C. Y., Slutsky, S. G., Richardson, S. C., Piper, R. C., Weisman, L. S. (2007). Loss of Vac14, a regulator of the signaling lipid phosphatidylinositol 3,5bisphosphate, results in neurodegeneration in mice. Proceedings of the National Academy of Sciences of the United States of America, 104(44), 17518-23. http://doi.org/10.1073/pnas.0702275104 
Zolov, S. N., Bridges, D., Zhang, Y., Lee, W.-W., Riehle, E., Verma, R., Weisman, L. S. (2012). In vivo, Pikfyve generates PI(3,5)P2, which serves as both a signaling lipid and the major precursor for PI5P. Proceedings of the National Academy of Sciences of the United States of America, 109(43), 17472-7. http://doi.org/10.1073/pnas.1203106109

Zoncu, R., Bar-Peled, L., Efeyan, A., Wang, S., Sancak, Y., Sabatini, D. M., Steinberg, B. E. (2011). mTORC1 senses lysosomal amino acids through an inside-out mechanism that requires the vacuolar H(+)-ATPase. Science (New York, N.Y.), 334(6056), 678-83.

http://doi.org/10.1126/science.1207056 


\subsection{APPENDIX C}

SUPPORTING INFORMATION FOR CHAPTER 4 

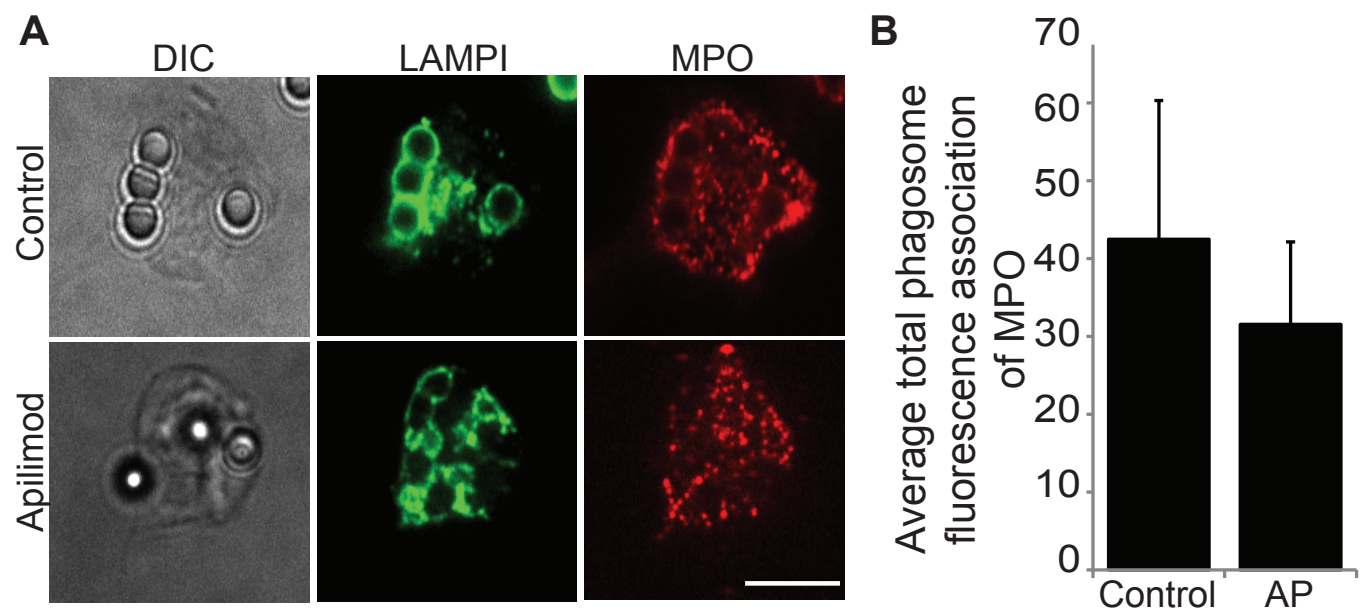

Figure S 4.1: PIKfyve inhibition does not block phagosome acquisition of MPO, a primary granule marker.

A. Neutrophils were treated with vehicle (control) or $20 \mathrm{nM}$ apilimod, followed by phagocytosis of IgG-coated beads and a chase of $1 \mathrm{~h}$. Cells were then stained for LAMP1 and MPO to detect lysosome and primary granule fusion with phagosomes. Scale bar $=10 \mu \mathrm{m}$ (add arrows). B. Quantification of phagosome-associated MPO fluorescence signal as described in Methods. Shown is the mean \pm SEM from $n=3$ experiments. Using Student's t-test, there was no apparent difference between control and apilimod-treated cells. 


\section{Chapter 5}

Concluding remarks and future directions 


\subsection{PIKfyve inhibition interferes with phagosome and endosome maturation in macrophages}

Control and prevention of infection remains a critical problem because many infectious agents have evolved strategies that circumvent the immune system. For example, Mycobacterium tuberculosis prevents fusion of phagosomes with lysosomes, thus protecting themselves from lysosomal degradation (Vergne et al., 2004). Therefore, it is very important to understand how phagosome maturation is being regulated in immune cells such as macrophages and neutrophils. In our study, we identified PIKfyve as a key regulator of phagosome maturation in macrophages and neutrophils, a lipid kinase that produces $\mathrm{PI}(3,5) \mathrm{P}_{2}$ and $\mathrm{PI}(5) \mathrm{P}$. We determined that in addition to phagocytosis, PIKfyve is required for phagosome maturation, in which cells depleted for PIKfyve failed to acquire lysosomal markers such as LAMPI and cathepsin D. Moreover, PIKfyve inhibition did not interfere with acquisition of PI(3)P; however, there was a delay in loss of PI(3)P from early phagosomes. In addition, inhibition of PIKfyve interfered with degradative capacity of lysosomes while it had no impact on lysosomal pH.

PIKfyve produces $\mathrm{PI}(3,5) \mathrm{P}_{2}$ and, directly or indirectly, $\mathrm{PI}(5) \mathrm{P}$. Therefore, to be able to determine which of these lipids regulates phagocytosis and phagosome maturation in macrophages and neutrophils, more studies will be needed to address this question. For instance, one can look at the dynamics of PIKfyve products, $\mathrm{PI}(3,5) \mathrm{P}_{2}$ and $\mathrm{PI}(5) \mathrm{P}$, during the process of phagocytosis and phagosome maturation using fluorescently labelled probes that bind specifically to $\mathrm{PI}(3,5 \mathrm{P})_{2}$ and $\mathrm{PI}(5) \mathrm{P}$. This will help visualize localization as well as changes in the level of each PIP during phagocytosis. However, to date, only few effector proteins have been identified to bind to $\mathrm{PI}(3,5) \mathrm{P}_{2}$ and $\mathrm{PI}(5) \mathrm{P}$ and the use of specific protein domain probes 
remains controversial to track these lipids in living cells. Therefore, future research trying to identify a probe that binds to these PIPs with high specificity will help us track changes in the level of these lipids during phagocytosis.

We speculate that $\mathrm{PI}(3,5) \mathrm{P}_{2}$ is required for phagocytosis to helps regulate TRPML1, a lysosomal calcium channel. Upon activation of TRPML1, calcium diffuses into the cytosol and high level of calcium near the site of internalization causes lysosomes to fuse with the plasma membrane. This fusion process helps deliver more membrane to the site of phagocytosis, which is required for phagocytic cup formation and internalization of the particle. Moreover, to understand how $\mathrm{PI}(3,5) \mathrm{P}_{2}$ helps control phagosome maturation, we tried to look at one of the downstream effectors of $\mathrm{PI}(3,5) \mathrm{P}_{2}$, TRPML1, and its role in phagosome maturation. TRPML1 was chosen to be one of the target downstream effectors of $\mathrm{PI}(3,5) \mathrm{P}_{2}$ since it is a lysosomal calcium channel and calcium is known to be a key regulator of membrane fusion.

\subsection{The phosphoinositide-gated lysosomal $\mathrm{Ca}^{2+}$ channel, TRPML1, is required for phagosome maturation}

Our results indicate that TRPML1 is required for phagosome maturation in macrophages in which loss of TRPML1 interferes with phagosomal fusion with lysosomes post-docking. In addition, we have shown that the cytosolic level of calcium increases during phagosome maturation. This calcium elevation that is involved in phagosome-lysosome fusion seems to originate from lysosome stores and not from the ER or extracellular space. We were able to show that phagosome maturation could be rescued in TRPML1 knock down macrophages by using the calcium ionophore, ionomycin, or over expressing TRPML1-wt. Performing these 
experiments side-by-side with PIKfyve inhibited cells, we observed a similar outcome in which phagocytosis and phagosome maturation were blocked in $\mathrm{PI}(3,5) \mathrm{P}_{2}$-deficient and TRPML1 knockdown macrophages. Therefore, we concluded that $\mathrm{PI}(3,5) \mathrm{P}_{2}$ might help regulate phagosome maturation in macrophages by activating the TRPML1 channel. The question that remains unanswered is that, if TRPML1 and $\mathrm{PI}(3,5) \mathrm{P}_{2}$ are always present on the lysosomal membrane, then why is TRPML1 not always open basal conditions. Therefore, further research is needed to look into the signaling cascade that coordinates TRPML1 binding to $\mathrm{PI}(3,5) \mathrm{P}_{2}$ with docking of phagosomes to lysosomes during phagosome maturation. We speculate that formation of the trans-SNARE complex may communicate with TRPML1 or PIKfyve activity to provide specificity to TRPML1 opening.

Membrane fusion requires $\mathrm{Ca}^{2+}$ and since there are many sources of $\mathrm{Ca}^{2+}$ such as the extracellular space, mitochondria, endoplasmic reticulum, and lysosomes. In this study, we showed that lysosomal $\mathrm{Ca}^{2+}$, not extracellular and $\mathrm{ER}$, is required during phagosome maturation. However, it is not well understood whether this increase in the level of $\mathrm{Ca}^{2+}$ is local, near the site of fusion and only involves those lysosomes that are fusing with the phagosome, or if a few lysosomes involved in phagosome maturation can trigger release of calcium from the entire lysosome population in the cell to elevate cytosolic $\mathrm{Ca}^{2+}$. To answer this question, lysosomal targeted genetically-encoded $\mathrm{Ca}^{2+}$ release sensors, GCaMP3-ML1 and LAMP1GCaMP3 (Shen et al., 2012), may be used in the future to measure local $\mathrm{Ca}^{2+}$ changes during phagosome-lysosome fusion. GCaMP3, is a genetically encoded $\mathrm{Ca}^{2+}$ sensor created from the fusion of calmodulin, GFP, and M13, a peptide sequence of myosin light chain kinase which in this case serves as the $\mathrm{Ca}^{2+}$-calmodulin binding domain. The GFP is circularly permutated in a 
way that creates a solvent pathway, which leads to protonation of the chromophore and poor absorbance of the light; therefore, leaving GFP with a poor fluorescent state. However, in the presence of $\mathrm{Ca}^{2+}$, calmodulin binds to four $\mathrm{Ca}^{2+}$ ions, causes a conformational change in calmodulin and the $\mathrm{Ca}^{2+}$-calmodulin complex binds to the M13 domain. This structural shift eliminates the water pathway in circular GFP and leads to de-protonation of the chromophore and emission of light (Figure 5.1 A) (Akerboom et al., 2009).
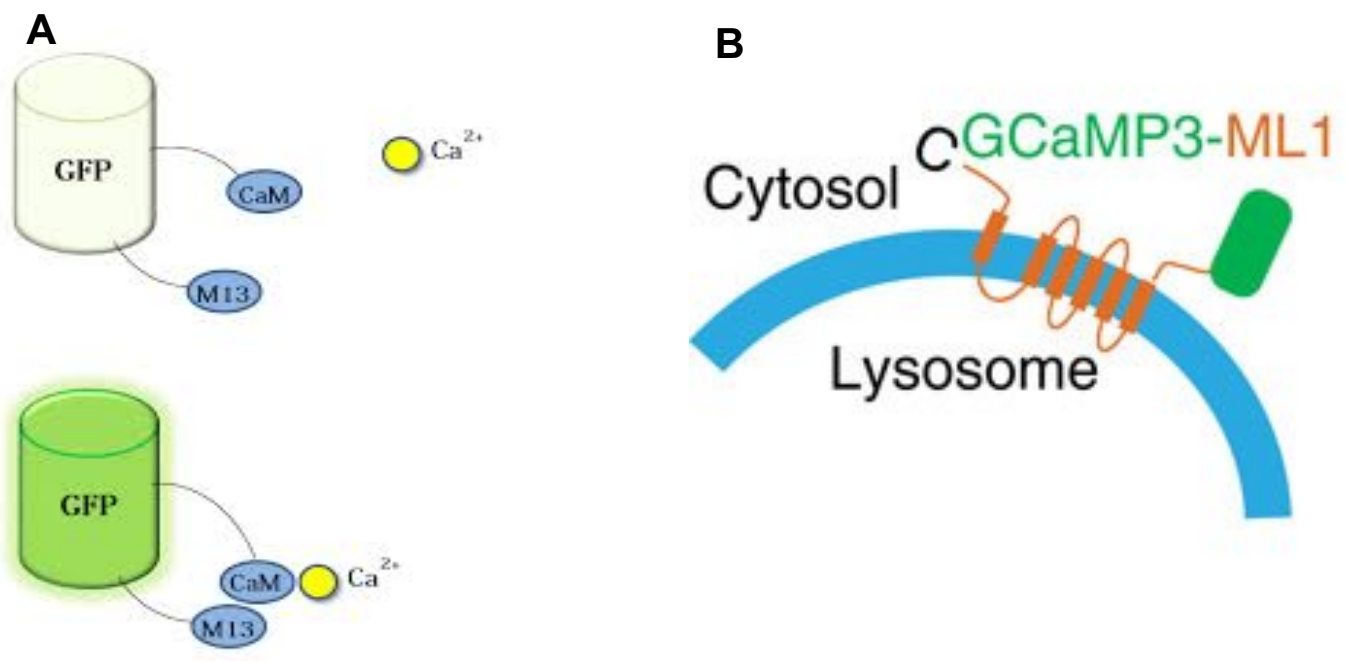

Figure 5.1: Lysosomes targeted genetically encoded $\mathrm{Ca}^{2+}$ sensors, GCaMP3-ML1.

A. Schematic diagram of GCaMP3, a $\mathrm{Ca}^{2+}$ sensing complex. In the absence of $\mathrm{Ca}^{2+}$, GFP is circularly permutated so the chromophore cannot be deprotonated, results in no fluorescence signal. High fluorescence signal is seen in the presence of $\mathrm{Ca}^{2+}$. B. structure of GCaMP3-ML1, GCaMP3 is fused to the N terminal of TRPML1 on the surface of the lysosome. Figure from (Shen et al., 2012)

GCaMP can be conjugated to the cytosolic $\mathrm{N}$-terminus of different proteins such as TRPML1 and LAMPI on the lysosomal surface (Figure 5.1 B). Upon transfection, GCaMP3-ML1 
and LAMP1-GCaMP3 will be expressed on the lysosomal surface, which helps to visualize and monitor lysosomal $\mathrm{Ca}^{2+}$ dynamics during phagocytosis and phagosome maturation. We can speculate that in $\mathrm{PI}(3,5) \mathrm{P}_{2}$-deficient cells TRPML1 remains inactive; therefore, no $\mathrm{Ca}^{2+}$ signal should be seen after phagocytosis in cells that are transfected with GCaMP3-ML1 or LAMP1GCaMP3. In addition, when TRPML1 knockdown cells are transfected with LAMP1-GCaMP3, there should be no $\mathrm{Ca}^{2+}$ signal after phagocytosis, explaining the block in phagosome maturation in TRPML knockdown cells.

In addition to fusion between phagosomes and lysosomes, PIKfyve and TRPML1 may also modulate phagosome fission and resolution, events that remain poorly understood. Indeed, phagolysosomes undergo a late stage of maturation characterized by fission (Krajcovic, Krishna, Akkari, Joyce, \& Overholtzer, 2013). We speculate that this fission plays a role in recycling material degraded within phagosomes, as suggested by Overholtzer's lab, but also in reforming lysosomes, for which there is a precedent in autophagy (Krajcovic, Krishna, Akkari, Joyce, \& Overholtzer, 2013).

During phagosome maturation, multiple lysosomes fuse with a phagosome; thus, the number of lysosomes in the cell decreases. Recent studies have tried to investigate the molecular machinery involved in autophagosome resolution and lysosome reformation. Autophagy, which is initiated during cell starvation, is a process in which cytoplasmic constituents are isolated within a double membrane vesicle called autophagosome. After maturation, their contents are degraded and recycled to support cellular anabolism. Autophagosome formation and maturation is followed by tubulation, budding and fission events, leading to the formation of small lysosomal-like organelles called proto-lysosomes, 
which then mature into functional lysosomes (Yu et al., 2010). Soon after tubulation, enrichment of $\mathrm{PI}(4,5) \mathrm{P}_{2}$ is seen on buds, which recruits adaptor protein 2 (AP2), necessary for the recruitment of clathrin, which plays a crucial role in budding and reformation of lysosomes (Rong et al., 2012).

Additional studies provided further understanding of the molecular machinery and signaling events during autophagic lysosome reformation. Degradation of autophagosomal contents provide nutrients and growth factors, that leads to the activation of mTOR, which in turn down regulates autophagy and triggers lysosomal reformation. Therefore, mTOR along with microtubules are shown to play an important role in lysosome reformation and homeostasis in the cell (Yu et al., 2010). In addition, fragmentation of phagolysosomes (containing apoptotic bodies) is known to be mTOR-dependent; after phagosome maturation, mTORC1 localizes on the surface of the phagosome, leads to fragmentation and distribution of phagosomal contents into lysosomal networks (Krajcovic et al., 2013). Thus, I propose that future work should explore the connection between PIKfyve and phagosome resolution and the reformation of lysosomes post-phagosome maturation.

This mechanism may have relevance for immunity but also tissue homeostasis since macrophages play an important role in clearing apoptotic and senescent cells. For example, red pulp macrophages internalize damaged/dying red blood cells (RBCs) into phagosomes, and after catabolism of hemoglobin molecules, the iron is recycled and returned to the bone marrow (de Back, Kostova, van Kraaij, van den Berg, \& van Bruggen, 2014). Regulation of this process is very important since iron recycling is necessary to maintain the production of new RBCs - an adult produces about $25 \times 10^{10} \mathrm{RBCs} /$ day (Gordon-Smith, 2007). In addition, 
disruption of this pathway leads to accumulation of dead RBCs in tissues that can cause inflammation. However, events that lead to resolution of the RBC-containing phagosomes and recycling of the phagosomal contents are not well studied. Therefore, future studies should focus on understanding the molecular machinery that controls phagolysosome resolution and lysosome reformation, which is very important to maintain tissue homeostasis. I postulate that PIKfyve may be necessary for this.

\subsection{The lipid kinase PIKfyve coordinates the neutrophil immune response through the activation of the Rac GTPase}

In addition to macrophages, neutrophils play an important role in our innate immune response; in fact, they are the first immune cells arriving at the site of infection for pathogen clearance. Neutrophils employ processes such as chemotaxis, phagocytosis, ROS production, phagosome maturation and degranulation to resolve infection and tissue damage. Here we show that among other regulators, PIKfyve plays a crucial role in neutrophil phagocytosis and phagosome maturation, possibly through the synthesis of $\mathrm{PI}(3,5) \mathrm{P}_{2}$ and the activation of lysosomal calcium channel, TRPML1. Indeed, our data support this model since we could rescue phagosome maturation in PIKfyve inhibited cells by using ML-SA1, a selective TRPML 1 agonist, as well as by increasing the intracellular calcium level using the calcium ionophore, ionomycin. These data indicate that lysosomal calcium release is necessary for phagolysosome biogenesis in neutrophils, indicating that macrophages and neutrophils employ PIKfyve and TRPML1 similarly to control phagosome maturation. 
In addition, inhibition of PIKfyve also interferes with ROS production as well as chemotaxis in neutrophils. Our work suggests that PIKfyve controls these processes by stimulating Rac GTPases, a major trigger for both chemotaxis and ROS. This is consistent with work by Oppelt et al. that showed that PIKfyve was important for cell migration of cancer cells (Oppelt et al., 2013, 2014). Our work does not address how PIKfyve helps stimulate Rac GTPases. We speculate that PIKfyve, through its lipid products, may recruit or activate a RacGEF, which replaces GDP for GTP. We suspect that Tiam1, a Rac-GEF, may be important in this process because Tiam1 is reported to bind PI(5)P, which can be directly or indirectly synthesized by PIKfyve. Future experiments should examine the membrane recruitment and localization of Rac GEFs such as Tlam1 in resting and activated neutrophils inhibited for PIKfyve. In addition, one could test if exogenously added $\mathrm{PI}(5) \mathrm{P}$ or $\mathrm{PI}(3,5) \mathrm{P} 2$ can stimulate Rac activity and ROS production in PIKfyve-inhibited cells. Since PIKfyve is involved in synthesis of both PI5P and $\mathrm{PI}(3,5) \mathrm{P}_{2}$, the addition of a specific PIP may help determine which lipid is important for which process.

Overall, in this thesis, I have collected a body of evidence supporting a critical role for PIKfyve, its lipid products and effector proteins such as TRPML1 in the regulation and function of phagocytosis, phagosome maturation and associated functions in macrophages and neutrophils. These cells are essential players in immune innate response, coordination of the adaptive immune response and in tissue homeostasis. It is tantalizing to consider the role of PIKfyve in immune-related functions including inflammatory damage of tissues since once of its most selective inhibitors, apilimod, was and is being clinically assessed as an anti-inflammatory agent and to fight cancer growth (Gayle et al., 2017). 


\subsection{References}

Akerboom, J., Rivera, J. D. V., Rodríguez Guilbe, M. M., Malavé, E. C. A., Hernandez, H. H., Tian, L., Schreiter, E. R. (2009). Crystal structures of the GCaMP calcium sensor reveal the mechanism of fluorescence signal change and aid rational design. Journal of Biological Chemistry, 284(10), 6455-6464. http://doi.org/10.1074/jbc.M807657200

Carstanjen, D., Yamauchi, A., Koornneef, A., Zang, H., Filippi, M.-D., Harris, C., Williams, D. a. (2005). Rac2 regulates neutrophil chemotaxis, superoxide production, and myeloid colony formation through multiple distinct effector pathways. Journal of Immunology (Baltimore, Md. : 1950), 174(8), 4613-20. http://doi.org/10.4049/jimmunol.174.8.4613

de Back, D. Z., Kostova, E. B., van Kraaij, M., van den Berg, T. K., \& van Bruggen, R. (2014). Of macrophages and red blood cells; A complex love story. Frontiers in Physiology. http://doi.org/10.3389/fphys.2014.00009

Gayle S, Landrette S, Beeharry N, Conrad C, Hernandez M, Beckett P, Ferguson SM, Mandelkern T, Zheng M, Xu T, Rothberg J, Lichenstein H. 2017. Identification of apilimod as a first-inclass PIKfyve kinase inhibitor for treatment of B-cell non-Hodgkin lymphoma. Blood. 129(13):1768-1778. doi: 10.1182/blood-2016-09-736892

Gordon-Smith, T. (2007). Red blood cells. Surgery (Oxford), 25(2), 57-60. http://doi.org/10.1016/j.mpsur.2006.12.004

Krajcovic, M., Krishna, S., Akkari, L., Joyce, J. a, \& Overholtzer, M. (2013). mTOR regulates phagosome and entotic vacuole fission. Molecular Biology of the Cell, 24(23), 3736-3745. http://doi.org/10.1091/mbc.E13-07-0408 
Rong, Y., Liu, M., Ma, L., Du, W., Zhang, H., Tian, Y., Yu, L. (2012). Clathrin and phosphatidylinositol-4,5-bisphosphate regulate autophagic lysosome reformation. Nature Cell Biology, 14(9), 924-934. http://doi.org/10.1038/ncb2557

Shen, D., Wang, X., Li, X., Zhang, X., Yao, Z., Dibble, S., Xu, H. (2012). Lipid storage disorders block lysosomal trafficking by inhibiting a TRP channel and lysosomal calcium release. Nature Communications, 3, 731. http://doi.org/10.1038/ncomms1735

Vergne, I., Fratti, R. A., Hill, P. J., Chua, J., Belisle, J., \& Deretic, V. (2004). Mycobacterium tuberculosis Phagosome Maturation Arrest : Mycobacterial Phosphatidylinositol Analog Phosphatidylinositol Mannoside Stimulates Early Endosomal Fusion, 15(February), 751760. http://doi.org/10.1091/mbc.E0

Yu, L., McPhee, C. K., Zheng, L., Mardones, G. A., Rong, Y., Peng, J., Lenardo, M. J. (2010). Termination of autophagy and reformation of lysosomes regulated by mTOR. Nature, 465(7300), 942-6.http://doi.org/10.1038/nature09076 